Improvement to the thermoelectric properties of PEDOT:PSS

\author{
क्षा

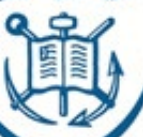 \\ Swansea University \\ Prifysgol Abertawe
}

\title{
Jonathan Atoyo
}

Primary Supervisor Matthew J Carnie

Secondary Supervisor Nicholas Lavery

Thesis submitted to Swansea University in fulfilment of the requirements for the Degree of Doctor of Philosophy $(\mathrm{PhD})$ SPECIFIC INK

College of Engineering Materials Research Centre 


\section{DECLARATION}

This work has not previously been accepted in substance for any degree and is not being concurrently submitted in candidature for any degree.

Signed ........Jonathan Atoyo..... (candidate)

Date .....30/09/2020.

\section{STATEMENT 1}

This thesis is the result of my own investigations, except where otherwise stated. Where correction services have been used, the extent and nature of the correction is clearly marked in a footnote(s).

Chapter 2.7 contains work published in literature. The section only contains the work done by Jonathan Atoyo ${ }^{1}$

Chapter 5 contains work that has been published in literature. All experimental work was conducted by Jonathan. James Mcgettrick helped to procure some XPS data, Matthew Carnie helped to edit the paper, and Matthew Burton helped to write parts of the paper. The work in Chapter 5 has been further edited. ${ }^{2}$

Other sources are acknowledged by footnotes giving explicit references. A bibliography is $\quad$ appended. Signed ....Jonathan Atoyo . (candidate)

Date $30 / 09 / 2020$

\section{STATEMENT 2}

I hereby give consent for my thesis, if accepted, to be available for photocopying and for inter-library loans after expiry of a bar on access approved by the Swansea University. Signed Jonathan Atoyo. (candidate)

Date $.30 / 09 / 2020$ 


\section{Acknowledgements}

I would like to acknowledge my supervisor Matthew J Carnie for choosing me for the $\mathrm{PhD}$ candidature and believing in me throughout the whole journey and being patient enough to allow me to explore research based on my interests. I have developed as a scientific writer because of his guidance. I have also come to understand the research process because of his instructions. Matthew R Burton has been an incredible help and has shown more kindness in my $\mathrm{PhD}$ than can be acknowledged. I have developed further as an analytical thinker.

Yahuwah (The Highest) has kept me in good health, and I would like to give glory to him.

I want to also acknowledge James Mcgettrick due to his exceptional kindness towards me during the early stages of my $\mathrm{PhD}$ candidature in facilitating my understanding the properties of $x$ ray photoelectron spectroscopy (XPS).

I would like to thank Tom Dunlop for similar reasons to James Mcgettrick.

Most importantly I want to thank my self for doing all the work lol.

I want to thank my mother for giving me life and all my friends including, Rollin Omari and Ikepanzi for keeping my mind occupied in abstractions about nature of reality as well as Madiop and Audra for helping me develop 2D and 4D thinking framework.

iii | P a g e 


\section{Abstract}

Thermoelectric materials can convert waste heat to electricity without moving parts. Extensive research into improving the efficiency of inorganic thermoelectric materials has allowed some materials such as bismuth tellurides to be commercialized. These materials, however, contain materials in low abundance on earth such as tellurium therefore their use and scaled production would be limited. Organic and hybrid thermoelectric materials can meet the gap for niche markets as well as be synthesized on mass, due to utilization of earth abundant elements such as carbon, sulphur, and nitrogen. The thermoelectric generators require several $\mathrm{n}$ and $\mathrm{p}$-type materials connected for optimal efficiency. There are many ways to create inorganic thermoelectric $n$ and p-types. However, for the organic thermoelectric materials the efficiency lags because of their lower electrical conductivity and Seebeck coefficient as well as the lack of effective strategies in the development of n-type materials.

Poly(3,4-ethylenedioxythiophene) polystyrene sulfonate (PEDOT:PSS) is one of the most successful and researched p-type organic thermoelectric material and thus, the thesis will explore effective strategies for decoupling the apparent trade-offs observed when improving either the electrical conductivity or Seebeck coefficient. The first major contribution to knowledge discussed in this study is the utilization of a reducing agent treatment on PEDOT-ionic liquid composites (reader is advised to refer to chapter 5). Another significant finding was the successful development of a novel route to an n-type single walled carbon nanotube, PEDOT:PSS composite (please refer to chapter 6). The final and most significant contribution to knowledge in this research project was the development of a set of novel single walled carbon nanotube, ionic liquid, PEDOT:PSS composites whereby after a post treatment with a guanidinium iodide in ethylene glycol solution allowed for improvement of the electrical conductivity from $3.4 \mathrm{~S} \mathrm{~cm}^{-1}$ to $3665 \mathrm{~S} \mathrm{~cm}^{-1}$ and Seebeck coefficient from $12 \mu \mathrm{V} \mathrm{K}^{-1}$ to $27 \mu \mathrm{V} \mathrm{K}^{-1}$ thereby leading to an optimised power factor of $236 \mu \mathrm{W} \mathrm{m} \mathrm{m}^{-1} \mathrm{~K}^{-1}$ at 140 ${ }^{\circ} \mathrm{C}$ (please refer to chapter 7 ). 


\section{Contents}

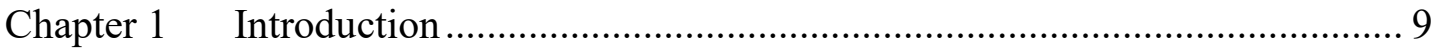

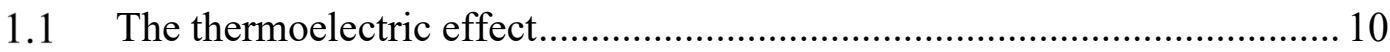

1.2 Fundamentals of thermoelectric materials.............................................. 12

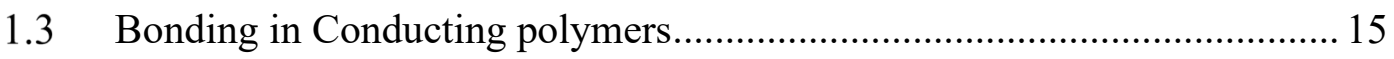

1.4 Charge carrier transport in organic semiconductors................................ 19

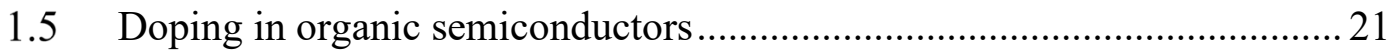

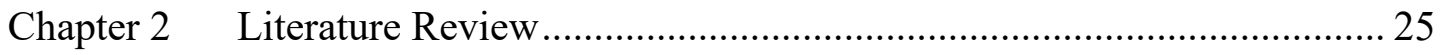

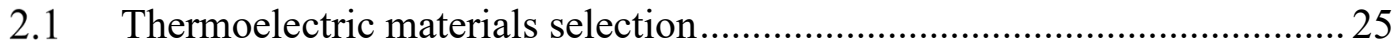

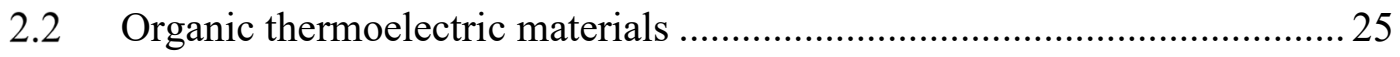

2.3 Improved PF through solvent engineering in PEDOT:PSS ...................... 28

2.4 Ionic-liquid treatment on PEDOT:PSS for improved PF .......................... 29

2.5 Improved PF in (OHTEGs) through nano-composition ............................. 30

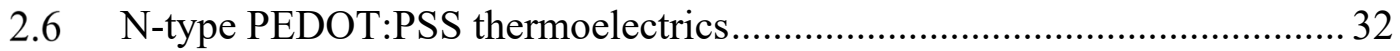

2.7 Graphene and CNT based PEDOT:PSS composites .................................. 34

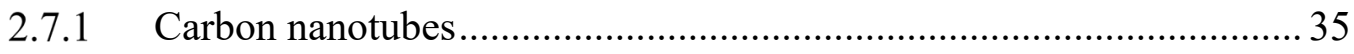

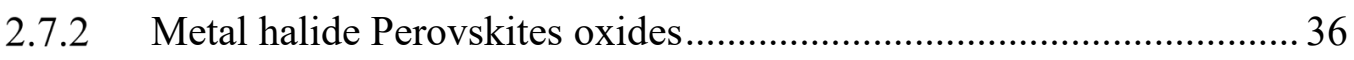

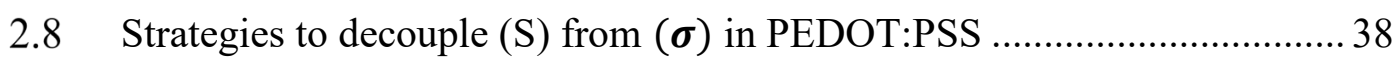

Chapter 3 Materials and Methods .................................................................. 41

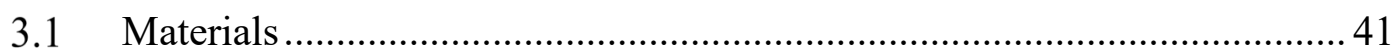

3.1.1 List of chemicals used in Chapter 4 (Effects of solvent treatment on PEDOT:PSS thin films)

3.1.2 List of chemicals used in chapter 5 (Enhanced Electrical Conductivity and Seebeck Coefficient in PEDOT:PSS via a Two-Step Ionic liquid and $\mathrm{NaBH}_{4}$ Treatment)

3.1.3 List of chemicals used in chapter 6 (Synthesis of $\mathrm{N}$ type SWNTPEDOT:PSS composites)

3.1.4 List of chemicals used in chapter 7 (Towards decoupled Seebeck coefficient from Electrical conductivity) ......................................................... 42

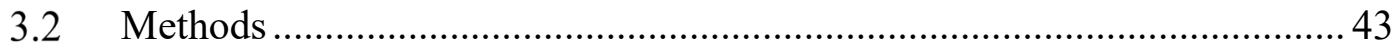

3.2.1 Fabrication of PEDOT:PSS based thin films ................................... 43

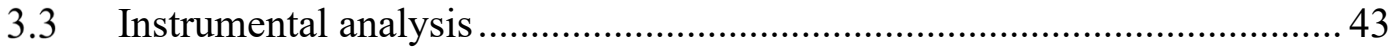

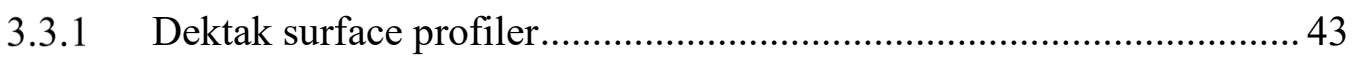

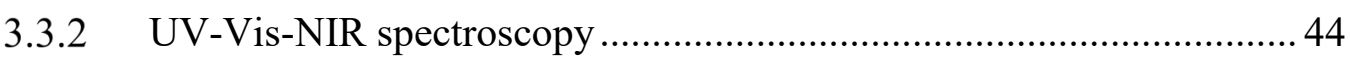

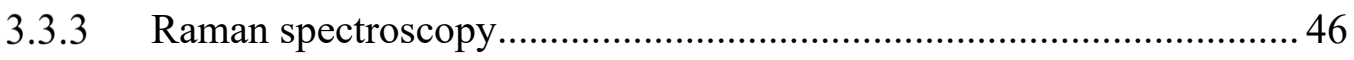


3.3.4 Xray photoelectron spectroscopy ….............................................. 46

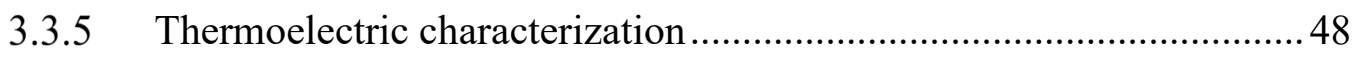

Chapter 4 Effects of solvent treatment on PEDOT:PSS .................................. 52

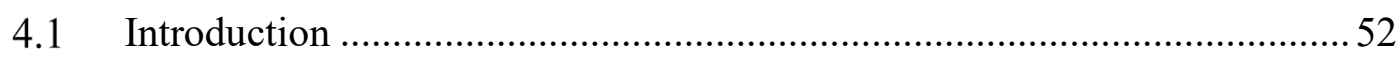

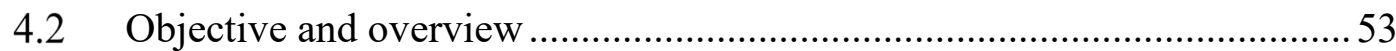

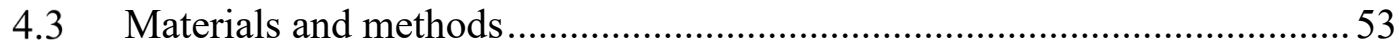

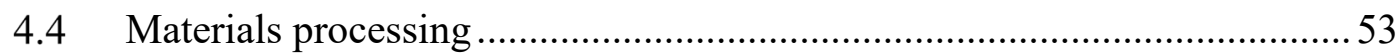

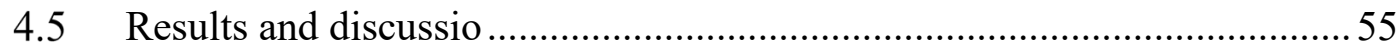

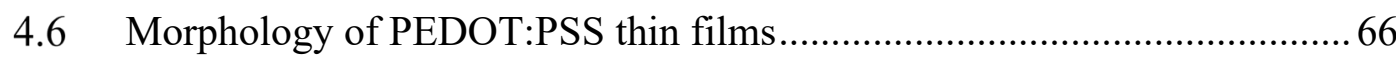

4.7 Molecular effects impacting electrical conductivity and Seebeck coefficient. 75

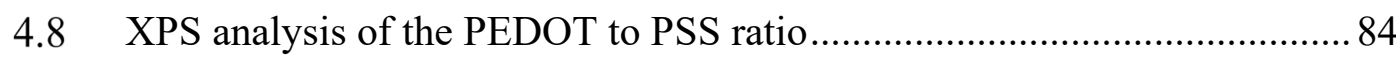

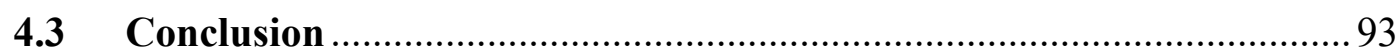

Chapter 5 Enhanced Electrical Conductivity and Seebeck Coefficient in PEDOT:PSS via a Two-Step Ionic liquid and NaBH4 Treatment........................... 95

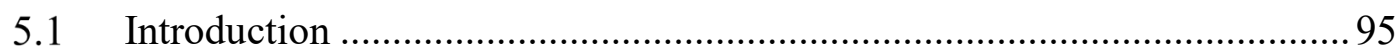

5.2 Fabrication of PEDOT:PSS EMIM:TFSI Composite Films ...................... 97

5.2.1 Preparation of EMIM:TFSI films................................................. 97

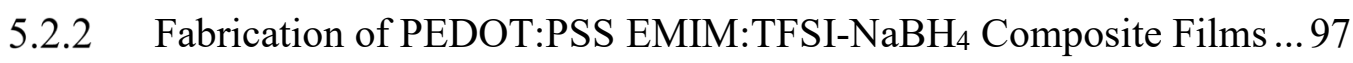

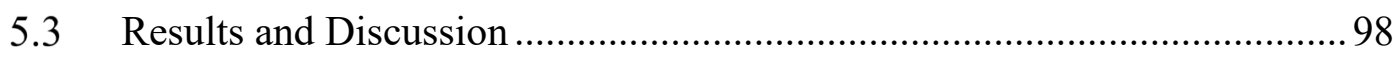

5.3.1 Thermoelectric Performance of PEDOT:PSS EMIM:TFSI Composite Films 98

5.3.2 Post Treatment of EMIM:TFSI Films with $\mathrm{NaBH}_{4} \ldots \ldots \ldots \ldots \ldots \ldots \ldots . . . . . . . . . . . .101$

5.3.3 Morphology of PEDOT/EMIM:TFSI composites ........................... 106

5.3.4 Mechanisms for the Improved Electrical Conductivity of EMIM:TFSI

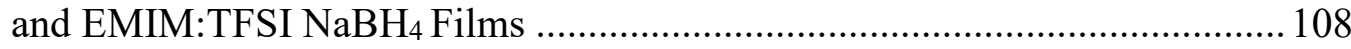

5.3.5 Mechanisms for the Improved Seebeck Coefficient of EMIM:TFSI

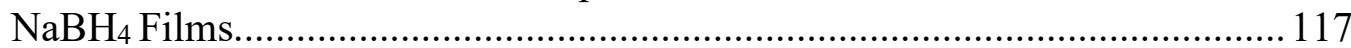

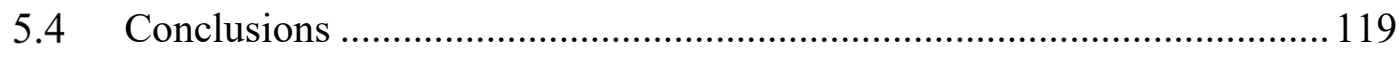

Chapter 6 Synthesis of N-type SWNT-PEDOT:PSS composites....................... 122

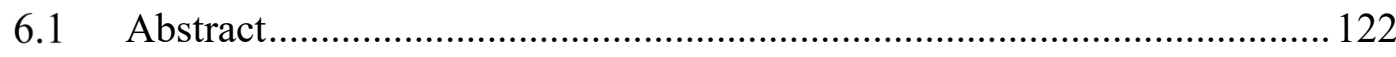

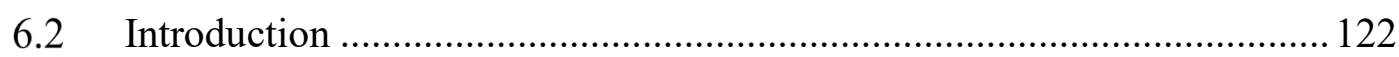

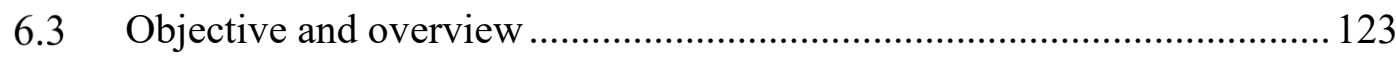

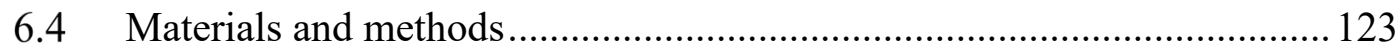

6.4.1 Synthesis of the fundamental controls ............................................. 123

6.4.2 Synthesis of n-type SWNT-PEDOT:PSS-conc films....................... 124 
6.4.3 Control for origin of n-type, films dubbed (SWNT-PEDOT:PSS-cont) 125

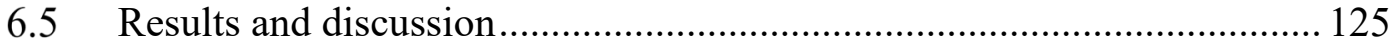

6.5.1 Preliminary experimental considerations ........................................ 125

6.5.2 Thermoelectric properties of SWNT-PEDOT:PSS-cont films .......... 128

6.6 Doping mechanism behind N-type characteristics of SWNT-PEDOT:PSS 136

6.7 Conclusion

Chapter 7 Towards decoupled Seebeck coefficient from electrical conductivity 147

7.1 Abstract 147

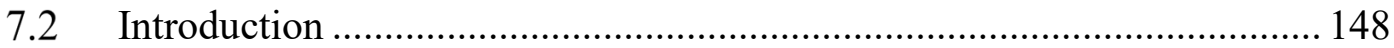

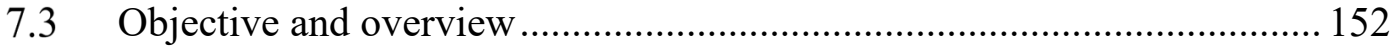

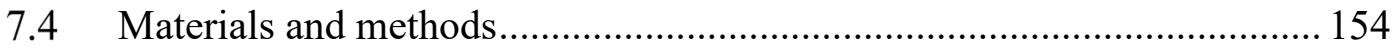

7.4.1 Synthesis of guanidinium iodide solution (GuI solution) .................. 154

7.4.2 Synthesis of PEDOT:PSS-EMIM:TFSI composites ......................... 154

7.4.3 Synthesis of SWNT-PEDOT:PSS composites.................................. 154

7.4.4 Synthesis of SWNT-PEDOT:PSS-EMIM:TFSI composites ............. 154

7.4.5 Spin coating of thin film composites ................................................ 154

7.4.6 Post treatment of thin film composite with guanidinium iodide solution 155

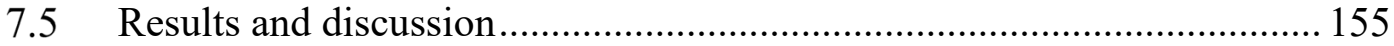

7.5.1 Thermoelectric properties of fundamental control composites.......... 155

7.5.2 Thermoelectric properties of guanidinium iodide treated films......... 159

7.5.3 Raman spectroscopy of the FC1, CX and SX films........................ 168

7.5.4 Proposed mechanisms for increased Seebeck coefficient.................. 174

7.6 Surface characterization of PEDOT:PSS films using XPS ..................... 187

7.6.1 Analysis of Carbon 1s spectra.......................................................... 187

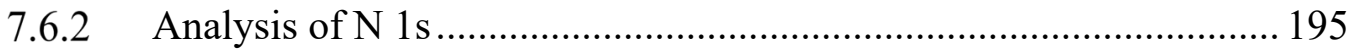

7.6.3 XPS quantification and desulphurization mechanism....................... 198

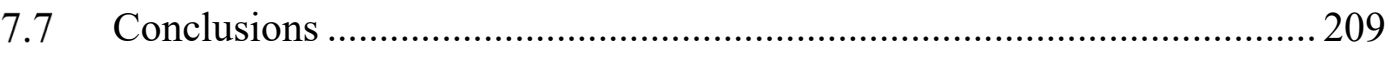

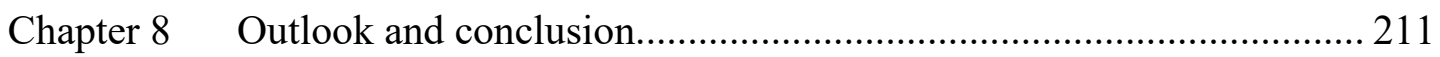

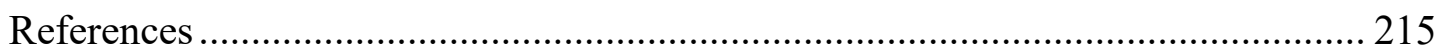


$\mathbf{8} \mid \mathrm{P}$ a g e 


\section{Chapter 1 Introduction}

As the earth's population continues to grow past 7.5 billion people as of 2019 with a corresponding evident growth rate over 1 percent annually, energy demands have become inculcated with geopolitical stability. ${ }^{3}$ Fossil fuels, which remain the largest energy source for most of the planet cannot sustain human civilizations indefinitely. ${ }^{4}$ Further still, because climate change concerns are also incontrovertibly linked to the use of fossil fuels due to their release of greenhouse gasses from incomplete combustion, alternative energy routes must be realized. ${ }^{5-7}$ Moreover, some products of combustion such as sulphur dioxides are linked to negative human health in dense city environment where it's concentrated. ${ }^{8}$ Consequently, these factors show the need for clean and renewable energy resources.

Thermoelectric generators (TEGs) which when made into functional devices known as Thermoelectric Devices (TED) are a class of materials with a lot of promise for application in clean and renewable energy. ${ }^{9-11}$ This is because majority of energy in most engines is dissipated as heat due to a conversion efficiency limits as described by the Carnot efficiency equation. ${ }^{12-16}$ As a waste heat recovery system TEGs do not, except for the initial materials synthesis add to the carbon footprint. They are solid state devices that can convert heat into electrical energy without moving parts. ${ }^{11,17} \mathrm{In}$ theory, this means the devices have lower failure potential than conventional engines comprised of multiple different moving parts requiring more significant emphasis on maintenance (assuming the TEG is stable and commercially operable). Subsequently, this unique factor increases the areas of application to include powering space probes and other devices utilized in deep-space exploration for missions that extend beyond our solar system whereby solar panels are impractical due to the low light intensity. ${ }^{18-}$ 21 


\subsection{The thermoelectric effect}

TEGs utilize a phenomenon known as the Seebeck effect which occurs when two dissimilar conductive materials are connected electrically in series and thermally in parallel. ${ }^{22-25}$ If the TEG is exposed to a temperature gradient $\left(\Delta \mathrm{T}=\mathrm{T}_{\text {Hot }}-\mathrm{T}_{\text {Cold }}\right)$ in which $\Delta \mathrm{T} \neq 0$ a voltage is generated due to the charge carriers moving from the hot side to the cold side as depicted in Figure 1.1.1. ${ }^{26,27}$

Typical device architecture requires $\mathrm{p}$ and n-type legs to maximise the efficiency. Further still, to make TEGs viable as energy recovery engines many of these legs must be connected in an optimal manner to maximise heat to electrical power conversion. ${ }^{28-}$ ${ }^{31}$ It's important to realize there are more considerations in the design of such systems like, the optimal leg length, geometry, number of legs, stability of the thermal paste applied in the synthesis of modules, as well as how many modules form a functional TEG unit etc. The p-type material has holes as the majority charge carrier meanwhile electrons are the majority charge carriers within n-type materials. ${ }^{27,32,33}$ As expressed in Figure 1.1.1, the holes and electrons will travel along the same direction away from the heat source towards the cold junction. As the carriers move along this path an electric field is generated whereby the current will flow.

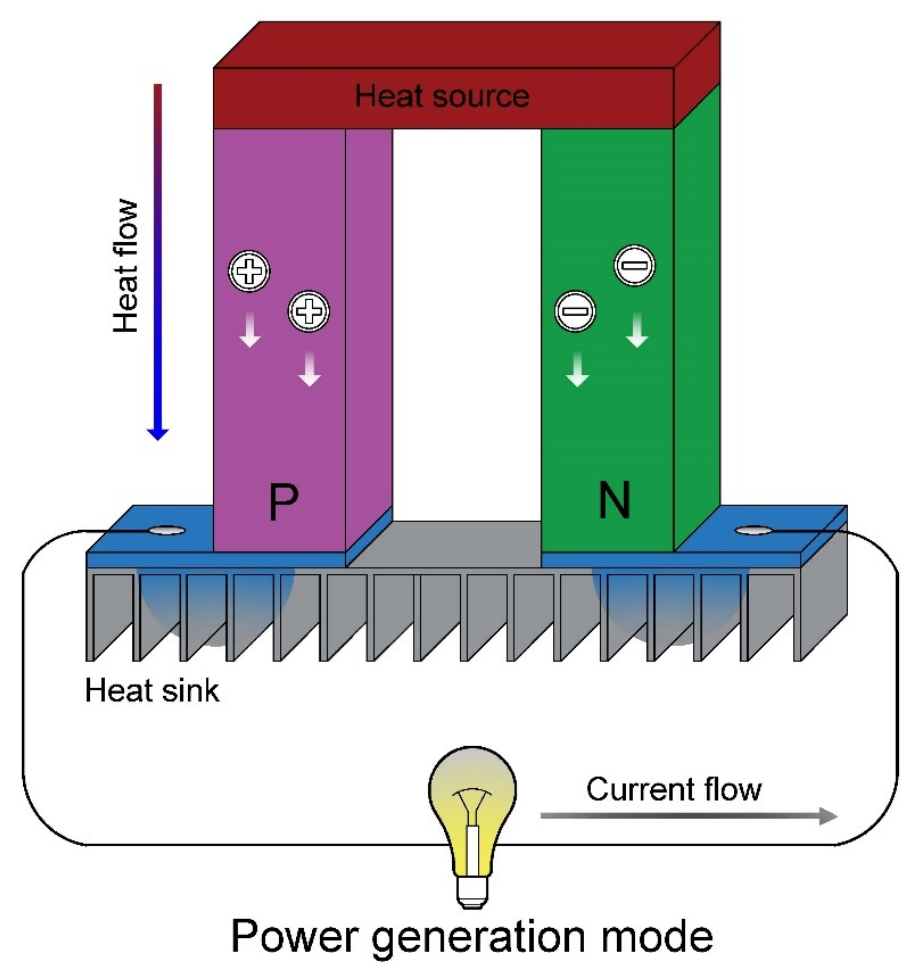


Figure 1.1.1, depicting flow of charge carriers in in the Seebeck effect. When there is enough difference in temperature $\left(\Delta T=T_{H o t}-T_{\text {Cold }}\right)$ between both junctions where $\Delta T$ is not 0 charge carriers ( $e^{-}$and $h^{+}$) will diffuse towards the cold end and create a voltage.

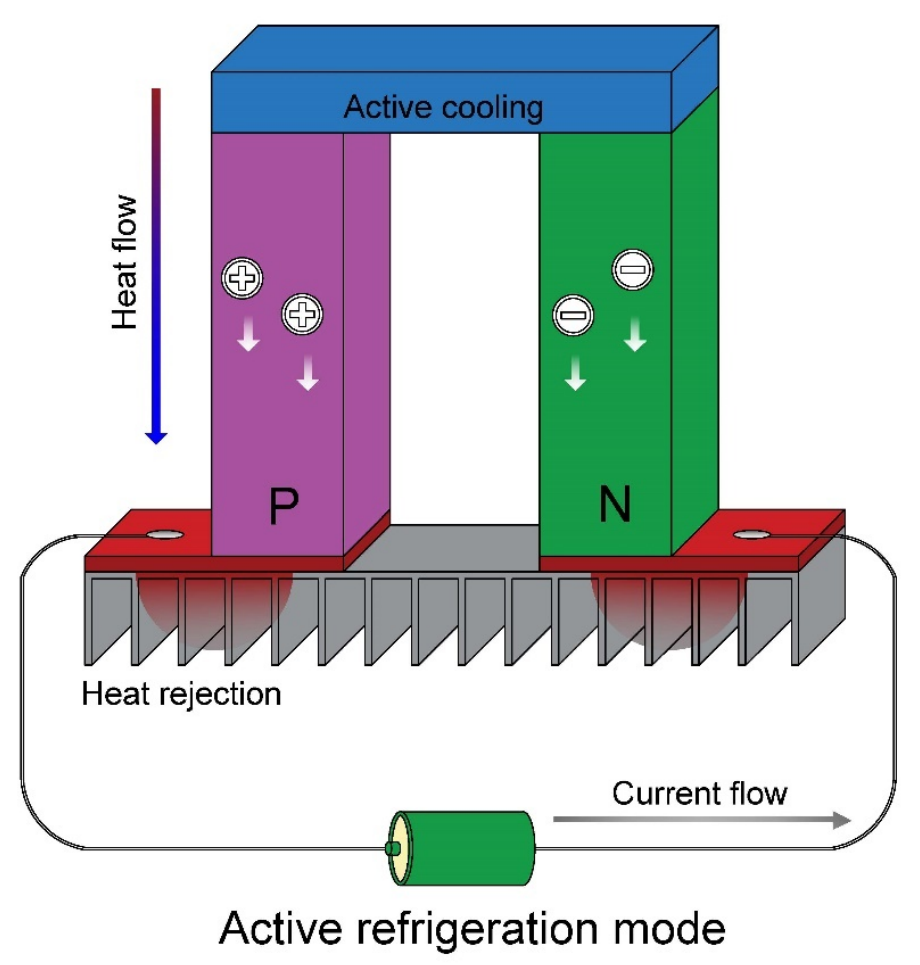

Figure 1.1.2. Schematic depicting flow of charge carriers in a Peltier effect system. When enough bias is applied (current) a temperature gradient will be generated and $\left(e^{-}\right.$and $\left.h^{+}\right)$will diffuse towards the hot junction and as consequence create an active cooling effect.

Figure 1.1.1 shows a power output to extract electricity generated by the Seebeck effect meanwhile Figure 1.1.2, represents a power input in the form of a battery. In this system, the reverse movement of charge carriers to the one described in Figure 1.1.1 will occur. In this scenario, the holes and electrons will actively flow away from one side and heat will be dissipated towards the other end and subsequently causing a temperature difference. ${ }^{34-36}$

This is known as the Peltier effect and can be used in cooling applications such as central processing unit (CPU) heat management, refrigerators, dehumidifiers, and cooling heat flux systems. ${ }^{28,37-41}$ However for this technology to replace more established power generators it would require higher efficiencies. The efficiency is 
denoted by a dimensionless figure of merit (ZT). Commercial TEGs operate at a ZT of around 1. A ZT of around 4 is required to reach this goal. ${ }^{42,43}$ The scope of this thesis is limited to the understanding of chemistry of optimised organic and hybrid thermoelectric materials (OHTEGs) as opposed to TED development.

\subsection{Fundamentals of thermoelectric materials}

For quantifying change and comparing performance within and between thermoelectric systems, the zT as expressed below in Equation (1) is used to denote thermoelectric efficiency of a material and or device. ${ }^{11,44,45}$ For thermoelectric devices (TED) however, a more complex set of equations that relate to Carnot efficiency, Thomson effect and transfer of heat and joules through circuits/materials are used to strategize device design for optimal performance. ${ }^{46-50}$

$$
z T=\frac{S^{2} \sigma T}{\kappa}
$$

Expressed in equation 1, S represents the Seebeck coefficient discussed in chapter 1.1. The electrical conductivity is $\sigma$, meanwhile $\mathrm{T}$ corresponds to the mean temperature between hot and cold end and $\kappa$ the thermal conductivity. ${ }^{51-53}$ For an optimally efficient TEG, the interplay between the Seebeck coefficient, electrical conductivity and the thermal conductivity must lead to a high ZT at the operating temperature. The Seebeck coefficient (S) is used to assess effectiveness of a specific material to generate voltage $(\Delta \mathrm{V})$ from the temperature gradient $(\Delta \mathrm{T})$. This relationship is expressed below in equation (2)

$$
S=\frac{-\Delta \mathrm{V}}{\Delta \mathrm{T}}
$$

The Seebeck coefficient is a materials property related to the rate of change of the density of states near the fermi level. ${ }^{54-56}$ Typically, metals have very high electrical conductivity because the Fermi level is within the conduction band and a much lower Seebeck coefficient because there are too many free mobile carriers as 
explained later in the Boltzmann transport theory (chapter 1.2 page 15) ${ }^{57-60}$ Ideal materials are therefore derived from semiconductors because the Fermi level or free mobile carrier population can be tuned through doping (defect engineering). ${ }^{61,62} \mathrm{An}$ insulator type material would ultimately yield an electrical conductivity that is too low, and a pure metal would usually have an extremely high thermal conductivity and low Seebeck coefficient whereby a low ZT would be realized. The electrical conductivity $(\sigma)$ gives information on the transport of the charge carriers electrons and holes $\left(\frac{e^{-}}{h^{+}}\right)$ respectively meanwhile $(\mathrm{T})$ depicts the absolute temperature. The thermal conductivity $(\kappa)$ expresses the transport of heat carriers namely phonons. A further analysis of $(\kappa)$ will lead to the expression in equation (3) below. ${ }^{63-65}$

$$
\begin{gathered}
\kappa=\kappa_{e}+\kappa_{l} \\
\kappa_{e}=L \sigma T
\end{gathered}
$$

The Lorenz number, L whereby $\left(L=2.44 \times 10^{-8} \mathrm{~W} \Omega \mathrm{K}^{-2}\right)$ is an approximated constant derived from some fundamental constants relating to kinetics of electrons and temperature. ${ }^{64}$ The approximate constant above is within the Somerfield limit for metals and heavily doped semiconductors but in some materials such as lightly dopped semiconductors and complex organic semiconductors it can deviate especially at moderate temperatures. Equation (3) shows that $(\kappa)$ is coupled to $(\sigma)$. The movement of phonons within vibrational lattice structure is described by $\left(\kappa_{l}\right)$ Thus, it's apparent that to improve ZT, studies must focus on decreasing $\left(\kappa_{l}\right)$ meanwhile maximising the numerator of equation (1). ${ }^{66-68}$

To further simplify equation (1) especially if $(\kappa)$ cannot be directly measured due to instrument limitations; if $(\kappa)$ is obtained in literature for the material in question then it can be estimated by taking the highest experimentally derived value for similar experimental parameters. The power factor $P F=S^{2} \sigma$, can be used as a tentative ZT alternative for efficiency considerations. ${ }^{69}$ This is not the only use for power factor as it can be useful in studying high temperature energy harvesting ${ }^{70}$ whereby a high temperature difference can be more easily maintained or potentially space applications since the difference in temperature between the radiation source and cold space is large enough that power factor would be more useful. ${ }^{72}$ Due to difficulties acquiring a reliable experimental method to determine $(\kappa)$ for thin film $(50-70 \mathrm{~nm})$ used in this research any ZT determinations were estimated through approximations of $(\kappa)$. 
Further still, PEDOT:PSS is the low $(\kappa)$ polymer matrix used in this study. It has been shown in literature that some nanocomposites rarely exceed much greater than $1 \mathrm{~W} \mathrm{~m}^{-}$ ${ }^{1} \mathrm{~K}^{-1}$ despite increased electrical conductivity. ${ }^{71}$

As denoted above in the ZT equation there is a competition between $(\kappa)$ and $(\sigma)$. If the $(\sigma)$ is increased then $\left(\kappa_{e}\right)$ will follow suit as described by the WiedemannFranz law thereby reducing ZT. ${ }^{73}$ However, it isn't only the interplay between PF and $(\kappa)$ that must be considered. The interrelation between $(\sigma)$ and (S) must also be ideal. This is because when broken down into subcomponents it becomes apparent that there are some contradicting partitions that are coupled that will reduce either $(\sigma)$ or $(\mathrm{S})$ as the other is increased. An equation relating to the Boltzmann transport theory as expressed in equation (4) can be used to described electronic transport within most solid-state materials including inorganic and to an extent organic crystal structured semiconductors like pentacene, carbon nanotubes PEDOT:PSS, polyaniline (PANI), etc. ${ }^{74}$ However, other models are often used in conjunction to this theory to analyse organic and hybrid thermoelectric systems as later explained in chapter 1.3.

$$
S=\frac{8 \pi^{2} k_{B}^{2}}{3 e h^{2}} m * T\left(\frac{\pi}{3 n}\right)^{\frac{2}{3}}
$$

Due to some part of the elements within the equations corresponding to, $(\sigma)$ and (S) being inversely coupled. ${ }^{74}$ Equation (5) below describes the interrelations further

$$
\sigma=n e \mu
$$

Within Equation (4) and (5) the Boltzmann constant is $k_{B}$, e conforms to the carrier charge, h corresponds to Planck's constant, meanwhile, $m$ is the effective mass of the charge carrier, $n$ denotes the carrier concentration, and $\mu$ the carrier mobility. ${ }^{75,76}$ Therefore, observing equation (1), (3), (4) and (5), one could formulate equation (6) as expressed below showing the full relationship between the relevant parameters regarding optimisation of $\mathrm{ZT}$

$$
Z T=\left\{\frac{8 \pi^{2} k_{B}^{2}}{3 e h^{2}} m * T\left(\frac{\pi}{3 n}\right)^{\frac{2}{3}}\right\}^{2} * \sigma / \mathrm{K}
$$


it's very clear a favourable strategy for improving ZT should focus on decoupling $\mu$ from $n$ thereby increasing carrier mobility as well as aforementioned $(\kappa)$ decoupling, whilst simultaneously finding an optimal carrier concentration that will favourably lead to both high $(\mathrm{S})$ and $(\sigma) .^{75,77-79}$

Therefore ideally, a highly electrically conductive materials with an optimal carrier concentration and highly disrupted phonon transport is desired. Such a material can be described by the phonon-glass crystal concept that can be formed in semiconductors such as skutterudites and clathrates whereby disorders can be introduced in the material to create a glass like structure to disrupt phonon transport meanwhile allowing for electron transport to be more crystalline to increase the electrical conductivity. ${ }^{80,81}$ Several studies show this concept is useful in selectively disrupting the phonon transport. ${ }^{82-85}$

A recent study achieved record high $\mathrm{ZT}$ across a wide range of temperature by improving the PF through $\mathrm{Na}$ doping and controlled growth of $\mathrm{SnSe}_{0.9} \mathrm{~S}_{0.1}$ single crystals and lowering thermal conductivity through structural band engineering and anharmonicity/point defects resulting in phonon scattering thus resulting in a ZT of 2.3 at $499.85{ }^{\circ} \mathrm{C} .{ }^{86}$

Other methods to scatter phonons have been demonstrated via nano structuring and quantum wells for inorganic TEGs. ${ }^{87-91}$ Superlattice strategies require specialized equipment to arrange atoms in a particular plane and precise patterning. Through nano structured engineering, the ZT of bismuth telluride/selenide-based TEGs has been significantly improved. Since its discovery, this material type has had its bulk ZT optimised to around 1 at room temperature but recent materials through nano structuration have managed to obtain ZT of above 1.4 for both $\mathrm{p}$ and $\mathrm{n}$-type derivatives. $^{92}$

\subsection{Bonding in Conducting polymers}

In polymers such as polystyrene sulfonate (PSS) the electrons in the carbon bonds pertaining to the backbone are $\mathrm{sp}^{3}$ hybridised. A two-dimensional model of the molecular structure of the polymer backbone is represented in Figure 1.3.1 below. 


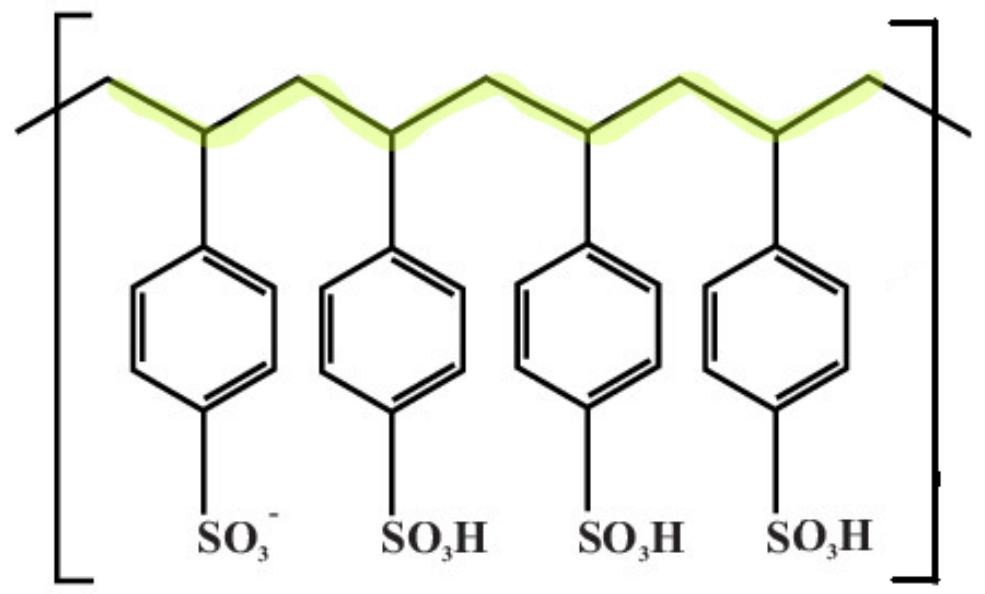

Figure 1.3.1 pictogram depicting 4 units of poly(styrenesulfonate) PSS. The highlighted section shows the nonconductive backbone of $s p^{3}$ hybridised carbon atoms.

The carbon atoms have strong stable covalent $\sigma$ bonds that prevent effective charge transfer between molecules. Due to the oxygen bonded to the sulphur atoms, when in an ionic solution with salts like $\mathrm{Na}^{+}$and $\mathrm{H}_{2} \mathrm{O} / \mathrm{H}_{3} \mathrm{O}^{+}$, it can be synthesized with poly(3,4-ethylenedioxythiophene) PEDOT to form PEDOT:PSS, which allows the otherwise water insoluble conductive polymer (PEDOT) to be solution processable. ${ }^{93,94}$ The mechanism of improved electrical conductivity via phase separation of PSS from PEDOT will later be explored in chapter 4.

In polymers such as PEDOT as represented in Figure 1.3.2, the carbon atoms on the backbone are $\mathrm{sp}^{2}$ hybridized. In this bonding situation, out of the four electrons in the outer shell of each carbon atom three are used to form 3 degenerate $\sigma$ bonding 
electrons in the form of, $1(2 s)$ and $2(2 p)$ orbitals. The remaining electron which is in an unhybridized (2p) orbital as depicted in Figure 1.3.3 is shared between

the same electron type in the adjacent carbon atom and forms the less stable higher charge density $\pi$ bond. These bonds are represented by a double bond as shown in the highlighted sections of Figure 1.3.2

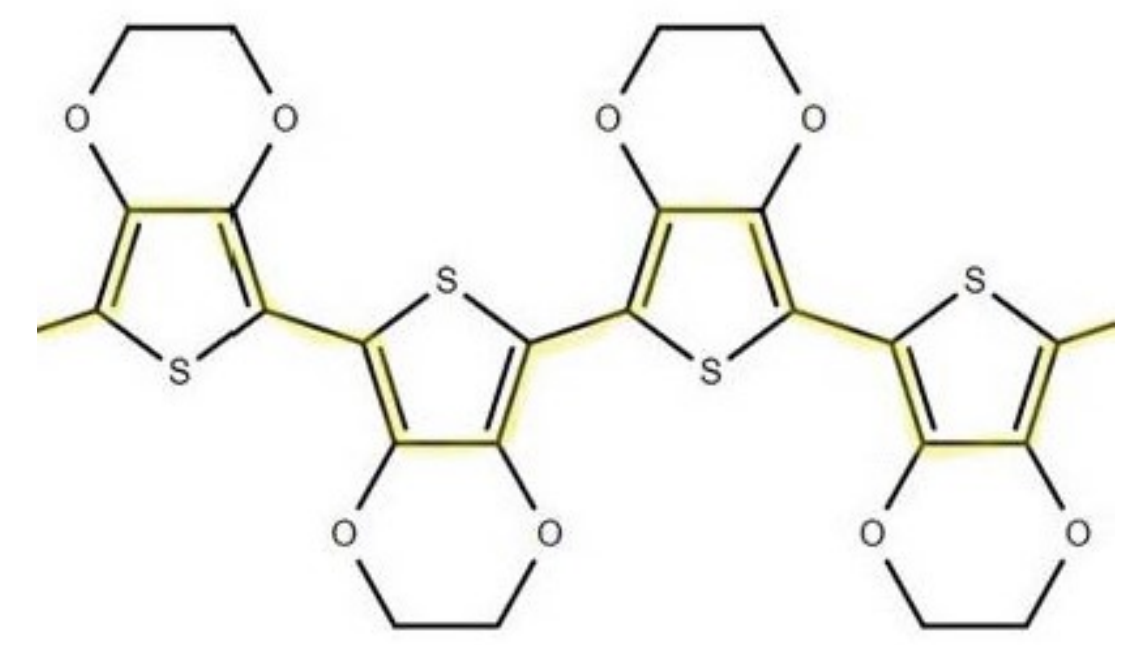

Figure 1.3.2. Pictogram representing the conductive pathway due to $\pi$ bonded electrons (highlighted) of the 4 repeated units of the polymer poly(3,4ethylenedioxythiohene) PEDOT.

Consequently, due to the weaker bond with higher charge density the $\pi$ bonds can break and reform in the adjacent carbon location which can cause a chain like reaction of bond breaking and bond forming and thus become delocalised across the molecule. 
Consequently, this creates a conductive pathway with an effective charge transfer potential relative to the $\mathrm{sp}^{3}$ bonds in Figure 1.3.1.

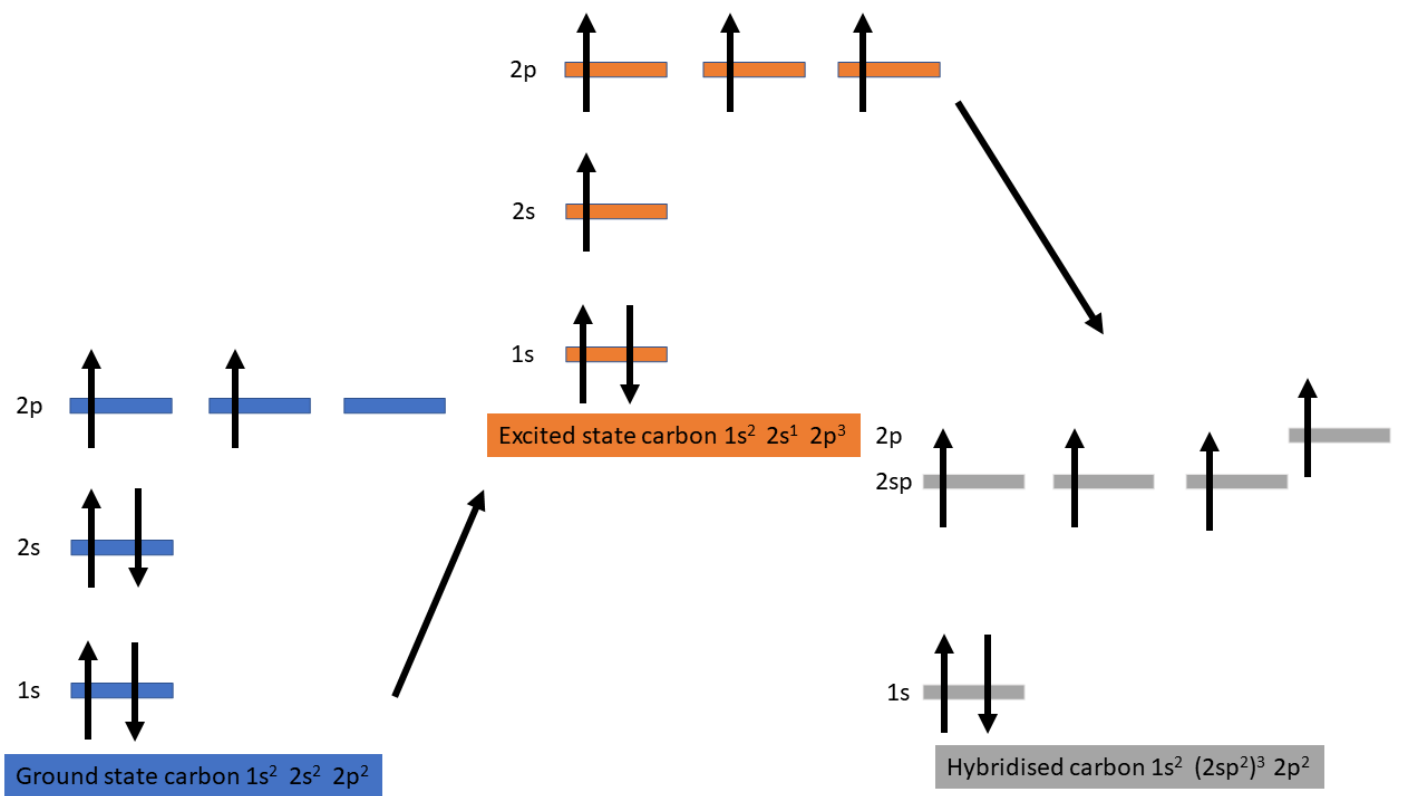

Figure 1.3.3. Colour coded pictogram depicting different electronic states in Carbon bonding orbitals. The ground state (furthest left) conforms to a simple unbonded carbon meanwhile the excited state (middle) depicts $s p^{3}$ hybridised carbon. The hybridised carbon (far right) depicts the $s p^{2}$ hybridised carbon with an unhybridized $2 p$ orbital.

In relation to Figure 1.3.1, in theory polystyrene should be able to conduct electricity due to the delocalised $\pi$ electrons in the benzene rings. Charge can build up in the benzene ring, however due to the $\mathrm{sp}^{3}$ backbone this charge will be trapped and statistically unable to hop onto the other carbon in the other benzene molecules to form a conductive circuit. If, however an intrinsically conductive material such as graphene is added into polystyrene it would allow the charge carrier build up in the benzene rings to be able to hop onto graphene nano particles and improve chances of it being carried across the system if it is energetically favourable. ${ }^{95}$ PEDOT is intrinsically undoped polymer therefore requires doping to improve the electrical conductivity. Therefore, there are effective strategies to improve the performance (conductivity)/ (charge accumulation at applied temperature difference) to improve the Seebeck 
coefficient of PEDOT for thermoelectric application. For the duration of this thesis the term thiophene/polythiophene will be used to refer to PEDOT chains.

\subsection{Charge carrier transport in organic semiconductors}

As expressed in chapter 1.3, the bonding induced by the overlapping of hybridised $\pi$ electrons create a band like delocalised carrier state. Organic semiconducting materials can be described as crystalline or amorphous depending on the degree of defects that affect their periodicity. One of the earlier transport theory for organic thermoelectric materials was proposed by Friedman based on the Boltzmann theory in organic crystal semiconductors. ${ }^{96}$ This transport theory uses the band like transport to describe mobility of charge carriers instead of focusing on transport as discrete unit cells as observed in other transport mechanism such as percolated hoping theory. In band theory, the generalized charge distribution around the valence band, Fermi level or conduction band is analysed to describe mobility. ${ }^{97}$ Due to the disordered imperfect crystal as seen in some polymers and small molecules the transport mechanism is more convoluted and difficult to describe than inorganic crystals therefore hopping mechanism is often used in conjunction to band theory to describe carrier transport. ${ }^{98-100}$

In organic semiconductors impurities can be introduced by grain boundaries dangling nonconductive bonds that disrupt conductive pathways or physical inclusions such as metals or nano powders, carbon nanotubes etc. ${ }^{101-103}$ A grain boundary refers to an interface between adjacent crystalline regions. ${ }^{102}$ Typically, these imperfections are described as static disorders since they are constant through time however when disorders are created through thermal fluctuations it can be described as dynamic disorders. Therefore, these thermal fluctuations may induce further effects on carrier mechanism in conjunction to the initial static disorders. ${ }^{104-108}$ This may result in higher or lower carrier mobilities at different temperatures. The change in enthalpy may influence the entropy of free carriers and induce a change in the charge localization in which incoherent tunnelling (hopping) may be statistically more likely or less likely depending on the distribution of charge carriers around the Fermi level.

Empirically, this dynamic disorder can be observed as a temperature dependence on the electrical conductivity. For example, in thermally activated/assisted 19|P a g e 
hopping mechanism, when observing a graph displaying electrical conductivity against temperature, a simultaneous increase in both temperature and electrical conductivity may be observed within the operating temperature range of the material. When treating different films with different chemicals sometimes there is an increase of the electrical conductivity as the measurement temperature increases. In these cases, the film can be described as thermally dependent electrical conductivity (thermally assisted hopping). If, however at higher temperatures, an increase in the electrical conductivity is not observed then it can be described as non-thermally dependent electrical conductivity (non-thermally assisted hopping). This has been described in literature as metallic conductivity. In this study non thermally, assisted hopping transport is used to include cases where the conductivity stays the same across the temperature range. This simplified model will allow inferences to be made regarding if and how temperature affects the electrical conductivity.

To determine if the material exhibits a hoping type mechanism the variable range hoping VRH (one among several) models can be used qualitatively to determine indicate carrier mechanism. ${ }^{98,109-111}$

When applying band theory to describe movement of carriers in organic systems, the highest occupied molecular orbital (HOMO) within an organic crystal is analogous to the valence band, meanwhile, the lowest unoccupied molecular orbital (LUMO) is analogous to the conduction band. Electrical conductivity (or hole conductivity in p-type materials) in organic semiconductors is allowable when the energy gap between these two bands is small enough for a charge carrier to transfer from the valence band to the conduction band. This can be occur at room temperature or via an excitation (photonic or thermal) to allow the transition. Doping may be utilized to introduce defects which subsequently may create alternative energy levels between the HOMO and LUMO, thereby reducing the band gap as there would be mid gap transitions thereby increasing the entropic distribution of carriers and thus increasing the statistical likelihood for some carriers to have enough energy to transition to the conduction band. ${ }^{112-114}$ 


\subsection{Doping in organic semiconductors}

Wide and mid gap band gap semiconductors cannot facilitate effective charge carrier across the material to create a current. This is contrasted by conductors which have an energetically more favourable band gap between the HOMO and LUMO thereby leading to intrinsically much higher electrical conductivity. ${ }^{115}$ Since the discovery of conductive organic semiconductors there have emerged several small molecules and conducting polymers that have been studied. ${ }^{116-120}$ However, due to the intrinsically low electrical conductivity, which being in part due to relationships between low carrier mobility and or carrier concentration, means most organic semiconductors require extra processing steps (doping) to increase conductivities. In pristine materials, this can be as low as $10^{-8} \mathrm{~S} \mathrm{~cm}^{-1}$ in small molecules to as high as $4000 \mathrm{~S} \mathrm{~cm}^{-1}$ in doped polymers like PEDOT:PSS. ${ }^{121-126}$ Therefore, it is possible to tune the conductivity of a polymer by controlling the dopant concentration within the material. In some cases, the dopant is a redox reagent (it will be referred to as dedoping when describing the reduction of $\mathrm{PEDOT}^{2+} / \mathrm{PEDOT}^{+}$molecules in this thesis). ${ }^{127-129}$ In inorganic semiconductors when an impurity with different electronic properties are introduced to a crystalline semiconductor the band gap can be tuned. For example, when $\mathrm{p}$ doping However, the doping scenario in organic semiconductors have multiple different mechanisms. An example is an integer charge transfer model whereby the addition of strongly electron donating n-type (reducing agent) or strongly electron withdrawing p-type (oxidising) molecule can inject or withdraw electrons respectively from the host material. ${ }^{130-133}$ Modelling a p-type dopant as an oxidising agent, it can be employed to a semiconductor to remove an electron from the highest occupied molecular orbital (HOMO) of the host material. Consequently, this would introduce some quantity of positively charged species (holes) across the conjugated backbone in the polymer. ${ }^{1}$ Along the same logic an N-type dopant can be modelled as a reducing agent which can transfer an electron to the LUMO of the corresponding organic material thereby inducing a negative charged carrier species (electrons) in the backbone. ${ }^{134-138}$ This model of charge transfer can be represented schematically as shown below in Figure 1.4.1 


\section{a)}

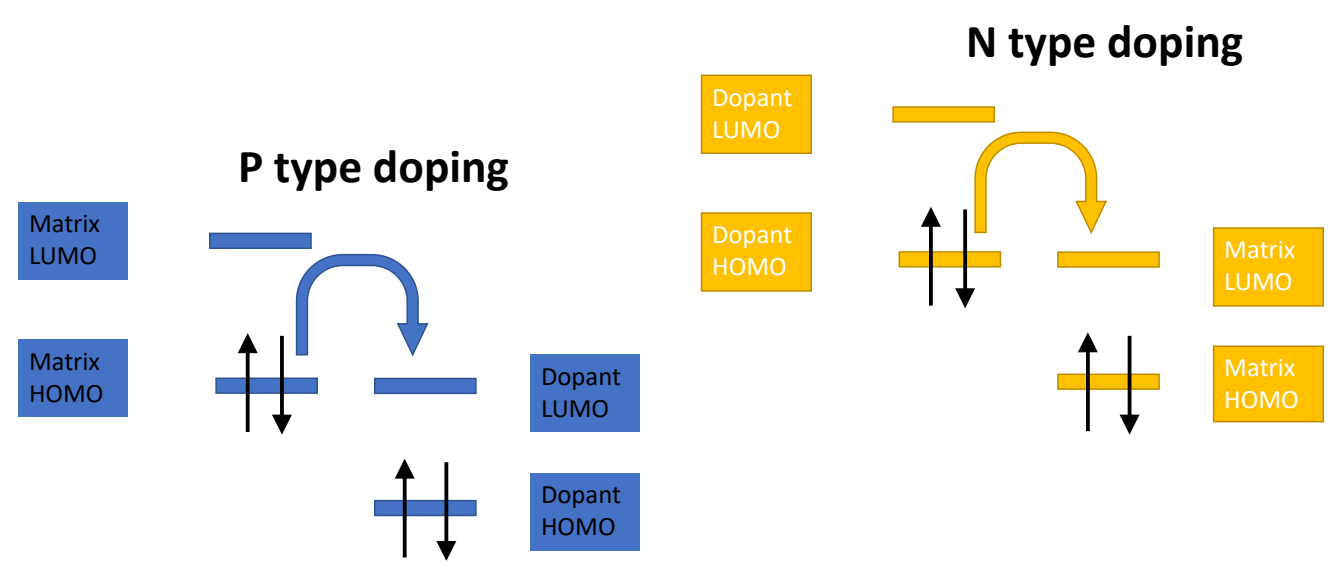

Figure 1.4.1 Schematic representing P-type (a), and N-type (b) doping mechanisms under the integer charge transfer model.

The choice of dopant requires an understanding of the redox potentials of both the dopant and the matrix material. To achieve p-type doping, the LUMO of the $p$ dopant must be at a lower energy than that of the matrix HOMO. In other words, the dopant must have a higher electron affinity than the matrix. ${ }^{1}$ For post doping matrix stability considerations it would be beneficial if the matrix forms a stable charged or neutral species after losing an electron. This can be achieved by utilizing counter ions or anions to counter the charge. In the inverse, (n-type) doping, the matrix must have a higher electron affinity and, if stability is important after doping, the material should be able to maintain the introduced negative charge without suffering from oxidation. ${ }^{139}$ A strategy to decrease the oxidation rate of an n-type carbon nanotube/graphene nano composite material was discussed in a paper by Cho et al. ${ }^{140}$ The team discovered that by forming highly aligned graphene oxide layers, the oxygen permeability rate would decrease enough to increase the number of days it would take for the films to lose their $\mathrm{n}$ type characteristics. This model has been used to describe some organic and hybrid semiconducting systems such as carbon nanotubes ( $p$ and n-type) as well as PANI, and PEDOT:PSS, (p-type), however for some materials such as PEDOT:PSS it's difficult to induce an n-type behaviour through doping of reduction without introducing other species in the matrix such a carbon nanotubes. ${ }^{141}$ The challenge to formulate $n$-type organic thermoelectric materials is of interest to the development of TEGs therefore it $22 \mid \mathrm{P}$ a g e 
will be the focus of an experimental section (chapter 6). ${ }^{138}$ As expressed by difficulty (experimentally) of producing an n-type PEDOT:PSS material while utilizing this integer charge transfer model, it's obvious that the model is incomplete in describing doping mechanisms in organic semiconductors. Other models such as hybrid charge integer have been posited as being more comprehensive. ${ }^{131,142-144}$

The hybrid charge transfer model explains doping of conducting molecules through the formation of charge transfer states induced from orbital mixing between donor and acceptor orbitals. This accounts for when the dopant remains in the materials (post doping). This hybrid charger transfer model can be used to explore charged counter ions and anions to the charged transfer states which the dopant itself may act as a stabilizer. ${ }^{131}$

Other doping strategies may include the imbedding of conductive nanomaterials within the polymer matrix. ${ }^{145,146}$ This approach would take the unique electronic qualities of the guest material into account and therefore by tuning the concentration of the guest material relative to the host matrix, it is possible to improve the Seebeck coefficient for example. ${ }^{147}$ In this case forming nanocomposites between the polymer and a material with intrinsically high Seebeck coefficient such as $\mathrm{SnSe}_{2}$, or other selenium, germanium, or tellurium nanoparticles/ flakes can improve the Seebeck coefficient of the bulk nanocomposite relative to the pristine polymer. ${ }^{148,149}$ The nano composition of tin selenide with PEDOT:PSS for example has been shown to improve the power factor and ZT as shown in a study by $J u$ et al, whereby a ZT of 0.32 was reached with a $20 \mathrm{wt} \%$ fraction of tin selenide to PEDOT:PSS. ${ }^{149}$ This was due to the extremely low and relatively unchanging thermal conductivity of $0.45 \mathrm{~W} \mathrm{~m}^{-}$ ${ }^{1} \mathrm{~K}^{-1}$ to the \% loading of the inorganic material as well as well as an increasing power factor in response to the tin selenide loading up to $20 \mathrm{wt} \% .{ }^{149}$ Thus, the band gap between the two materials is important to allow charge transport within the composite. This is an effective method for producing n-type conducting polymers. Because of the ionic properties of ionic liquids some studies have realized n-type characteristics in conducting polymers by using the anionic component to induce n-type characteristics by charge injection into the polymer meanwhile the imidazolium cation part acts as a charge stabilizer to prevent rapid air re-oxidation. ${ }^{150}$ An example of this is the use of 1-ethyl-3-methylimidazolium ethyl sulfate on polyaniline (PANI) and obtained a 
Seebeck coefficient of $-138.8 \mu \mathrm{V} \mathrm{K}^{-1}$ of which the n-type characteristics were maintained for 15 days due to the imidazolium cation balancing the negative charge. ${ }^{150}$ 


\section{Chapter 2 Literature Review}

\subsection{Thermoelectric materials selection}

The process of materials selection as well as strategies to improve favourable thermoelectric parameters should focus on cost effectiveness, ease of processability, low net-negative environmental impact, use of renewable re-agents (atom economy), and must have potential for mass production by using earth abundant, low toxicity elements which are stable at optimal operating temperature, light, and humidity etc. ${ }^{17,151-153}$ Some issues with some of the latest ways to improve ZT involve complex muti-step processes that would contradict some of the factors listed above. Spark plasma-sintering can lead to high power factor for inorganic TEGs, by improving electrical transport through increased crystallinity and control of the vibrational phonon paths through scattering induced controlled defects. ${ }^{154,155}$ Controlled crystal growths, quantum confined phonons, superlattice structures and molecular ordering, are great examples of effective strategies that have shown to improve said factors. ${ }^{156-}$ 161

Nano structuration has been shown to improve ZT as previously explained and when compared to some of the methods listed above it can require much less specialized equipment. ${ }^{162}$ hydrothermal, Solvothermal and other simple solution processing techniques will be used in conjunction to nanostructured films for this study with emphasis on chemical treatment. ${ }^{163-165}$

\subsection{Organic thermoelectric materials}

Organic electronics is a growing field in semiconductor solid state physics/chemistry and materials science due to some intrinsic advantages pertaining to organic materials such as an earth abundant atom economy (carbon based). ${ }^{166,167} \mathrm{In}$ comparison, inorganic semiconductors often use derivatives of less abundant metals such as selenides, gallium, germanium etc. ${ }^{168-170}$ This means if organic materials overcome some intrinsic issues such as thermal, air, water, and mechanical stability as 
well efficiency concerns then they can compete with and potentially replace some traditional inorganic based semiconductors. ${ }^{69,171,172}$ At the very least it would mean organic thermoelectric materials can be used in niche applications such as wearable energy harvesting devices or low energy medical devices etc. ${ }^{17,11,166,173}$ Among the most studied materials include; polyanilines, 5.6.5 1,2-Dithioles and Dithiolanes, carbon nanotubes, (CNT), polyacetylenes, and polythiophenes such as Poly(3,4ethylenedioxythiophene)poly(4-styrenesulfonate) (PEDOT:PSS). ${ }^{17,36,111,174,175}$

PEDOT:PSS has been widely studied in an array of organic electronics technologies such as photovoltaics (PVs), in which certain grades of PEDOT:PSS is used as the hole transport material or in some cases as a replacement for indium tin oxide (ITO) as the conductive electrode in PVs or liquid clear display systems. ${ }^{176-181}$ PEDOT:PSS has also been used in other applications such as organic light emitting diodes (OLED) capacitors and so on. ${ }^{182-186}$ It's been extensively studied (in conjunction with nano composites) as an (OHTEG) material since it was discovered that it's electrical conductivity can be drastically increased without significantly affecting the phonon contribution of $(\kappa)$ via solution mixtures and post treatments with high dielectric-constant polar co-solvents. ${ }^{187,188}$ In a study by Liu et al. ${ }^{191}$ it was discovered that in a PEDOT:PSS film treated with DMSO that the electronic contribution increased more so than from the phonon contribution to the thermal conductivity as estimated through Wiedemann-Franz law in which it was found that the data was closely related with the Sommerfeld value of the Lorenz number. This infers that although the total $(\kappa)$ in organic thermoelectric materials may not increase as much as for some inorganic materials that there would still be an increase. The polymer is constituted by repeated units of $\mathrm{EDOT}^{+}$ionomers and $\mathrm{PSS}^{-}$counter ions to stabilize charge distribution and allow PEDOT:PSS to be water soluble since PEDOT is hydrophobic. ${ }^{189}$ The excellent properties such as, water solubility, processability, ease of printing allows for a wide range of environmentally friendly synthetic routes to desired performance and device fabrication. ${ }^{94,166,190,192,193}$

The term primary doping is used to describe the "as prepared" PEDOT:PSS solution due to the oxidative polymerization method used during its synthesis. Without further doping or dedoping however, the PF of $0.07 \mu \mathrm{W} \mathrm{m}{ }^{-1} \mathrm{~K}^{-2}$ is observed in which it is too low to be useful in commercial applications. ${ }^{126,194}$ In order to improve the thermoelectric performance of PEDOT:PSS, effective chemical treatment is usually 
ascribed as secondary doping (for improvement of electrical conductivity) meanwhile dedoping (is used to tune the Seebeck coefficient) to optimise the PF. ${ }^{195}$ The term secondary doping relates to the use of doping strategies that does not necessarily change the oxidation state of PEDOT, often involves solvents treatment. ${ }^{196}$

Another desirable property of PEDOT:PSS used as an organic thermoelectric material is due to its intrinsically low thermal conductivity in the range between 0.1 $0.3 \mathrm{~W} \mathrm{~m}^{-1} \mathrm{~K}^{-1} 67,197$. Further still, the p-type polymer is resistant to thermal degradation from a range below room temperature to temperatures reaching $180{ }^{\circ} \mathrm{C} .{ }^{18,198}$ At temperatures above $230^{\circ} \mathrm{C}$ a decrease in the work function as measured by ultra violet photoelectron spectroscopy (UPS) can be observed in which with a corresponding decrease in electrical performance indicates a Diels-Alder reaction ( $\mathrm{SO}_{2}$-extrusion) occurring whereby thiophene units in PEDOT:PSS break down. ${ }^{31}$ This makes this material a great candidate for energy recovery low and medium energy recovery application but not high temperature applications. There is however a lack in research progress into the n-type form of PEDOT:PSS and currently there are only some successful synthetic routes, such as imbedding carbon nanotubes or tellurium derivatives within the polymer matrix. ${ }^{199,200}$ When treated with a reducing agent the majority charge carriers in the carbon nanotubes may change from holes to electrons in which through $\pi-\pi$ stacking with the PEDOT:PSS an overall electronic n-type charge transport is observed in the nancomposite. ${ }^{201}$ The p-type PEDOT:PSS is relatively stable in air and in some cases its materials properties can be altered to increase water stability as seen in literature. ${ }^{202}$ In a recent study the water resistance of a PEDOT:PSS composite was increased via cross linked synthetic route to a PEDOT:PSS-bisphenol diglycidyl ether film. The composite proved to also be resistant to significant electrical deformation as well as improved thermal stability. ${ }^{203}$

Because of its highly tuneable doping states (oxidation states ), it's possible to obtain desired PF through a variety of techniques such as post drop coating chemical treatment on a thin film, to more complex strategies such using electrochemistry to deposit PEDOT nano particles on a substrate. ${ }^{204,205}$ Within a typical electrochemical set up there is an electrolyte solution with desired ions for deposition as well as 3 electrodes including a reference, working and counter electrode. ${ }^{206}$ By varying the electrode potential, the redox state and or the deposition conditions can be induced. It has been shown that the oxidation state in this polymer is directly related to $(\sigma)$ and 
(S) in which is usually coupled. ${ }^{206}$ Dedoping usually describes reducing the thiophene units since its doped state is highly oxidised. Strategies to control these states and decouple $(\sigma)$ and $(\mathrm{S})$ would yield the highest $\mathrm{PF}^{207}$ The low $(\kappa)$ appears to be decoupled from the PF as the $(\kappa)$ remains relatively low at around $1 \mathrm{~W} \mathrm{~m}^{-1} \mathrm{~K}^{-1}$ despite improvements in $(\sigma)$ and $(\mathrm{S})$ even when made into nanocomposites by high loading of up to $90 \%$ by weight carbon nanotubes. ${ }^{71,170}$ This is of interest because carbon nanotubes have been shown to exhibit very high $(\kappa)$ without being encapsulated within a polymeric matrix. ${ }^{22,208}$

\subsection{Improved PF through solvent engineering in PEDOT:PSS}

Through secondary doping with polar solvents, acids, and salt solutions, has been shown to increase significantly. ${ }^{128,209}$ Conductivities of $620,640,800,1300$, and $1900 \mathrm{~S} \mathrm{~cm}^{-1}$ have been achieved by treating PEDOT:PSS with dimethyl sulfoxide (DMSO), ethylene glycol (EG), polyethylene glycol, methanol and formic acid, respectively. ${ }^{210,211}$ However, although this method improves the $\sigma, \mathrm{S}$ is not significantly affected because the as mentioned solvents do not reduce the oxidation state of PEDOT:PSS. Nonetheless, because the $\sigma$ is increased, the PF derived has been shown to be significantly higher than that of pristine PEDOT:PSS $\left(0.067 \mu \mathrm{W} \mathrm{m}^{-1} \mathrm{~K}^{-2}\right)$. When a solution of PEDOT:PSS is treated with DMSO and spin coated into a thin film, a PF of $18.2 \mu \mathrm{W} \mathrm{m}{ }^{-1} \mathrm{~K}^{-2}$ is observed. Utilizing a post treatment however, in which DMSO is drop casted onto the PEDOT:PSS, a much higher PF of $\left(30.1 \mu \mathrm{W} \mathrm{m}{ }^{-1} \mathrm{~K}^{-2}\right)$ is observed. ${ }^{212}$ Experiments conducted have proved the potential to simultaneously increase the electrical conductivity and Seebeck coefficient of PEDOT: PSS whilst at the same time decreasing the $\mathrm{k}_{1}$ (vertical) thermal transport phenomena. ${ }^{213}$ Ultimately, this led to an overall increase in the power factor and a maximum ZT of 0.42 nearing that of inorganic semiconductor thermoelectric materials. A Seebeck coefficient reaching up to $70 \mu \mathrm{V} \mathrm{K}^{-1}$ and a power factor reaching $469 \mathrm{uW} \mathrm{m}^{-1} \mathrm{k}^{-2}$ using ethylene glycol as a secondary post treatment dopant on a spin casted film comprising made from a mixed 5\% v/v DMSO to PEDOT solution in an oxygen free environment. (This result has not been corroborated by other researchers however and remains controversial). 
Other groups have managed to increase the PF by post treating PEDOT films with $\mathrm{H}_{2} \mathrm{SO}_{4}$ and other strong acids to selectively strip the insulating constituent PSS and allow for a more ordered conformation of the conductive PEDOT component. ${ }^{121,126,210,214}$ As reported, the corresponding electrical conductivity on pristine PEDOT: PSS reached a maximum of $4839 \mathrm{~S} \mathrm{~cm}^{-1}$ for undiluted strong acid treatment following from a pure methanol pre-treatment. ${ }^{121}$ However, what is notable is that the Seebeck coefficient decreased from $19.4 \mu \mathrm{V} \mathrm{K}^{-1}$ to $10.35 \mu \mathrm{V} \mathrm{K}^{-1}$, leading to an optimized PF of $51 \mu \mathrm{W} \mathrm{m} \mathrm{m}^{-1} \mathrm{~K}^{-2}$. Furthermore, the research group synthesized PEDOT: tellurium nanowires and treated the film with $\mathrm{H}_{2} \mathrm{~S}_{4}$. It was observed that the PEDOT: tellurium composites had a low electrical conductivity of $19 \mathrm{~S} \mathrm{~cm}^{-1}$ meanwhile maintaining a high Seebeck coefficient of $250 \mu \mathrm{V} \mathrm{K}^{-1}$ thus, leading to a PF of $70 \mu \mathrm{W} \mathrm{m} \mathrm{m}^{-1} \mathrm{~K}^{-2}$ giving a ZT of 0.1 . The group altered the treatment conditions and realized by varying $\mathrm{H}_{2} \mathrm{SO}_{4}$ concentrations a power factor of $284 \mu \mathrm{W} \mathrm{m}{ }^{-1} \mathrm{~K}^{-2}$ could be realized. This optimized power factor accounted for a reduced Seebeck coefficient of $114 \mu \mathrm{V} \mathrm{K}^{-1}$ and an increased electrical conductivity of $214 \mathrm{~S} \mathrm{~cm}^{-1}$. Thermal conductivity measurements from a different research group for a similar material was used to estimate a $\mathrm{ZT}$ of 0.39 . This is not a reliable method for obtaining $\mathrm{ZT}$ as the true thermal conductivity is unknown and could be much higher. A high ZT of 0.54 for a Te-PDOT:PSS material was obtained by a group studying the effects of the size of a single core/shell nanowire of the material affects the thermoelectric properties. ${ }^{215}$ It was discovered that by reducing the diameter of the nanowire that the electrical conductivity would increase while the thermal conductivity would decrease. Due to a Seebeck coefficient that was not affected significantly at $400 \mathrm{~K} 0.54 \mathrm{ZT}$ was calculated. It is of note that due to the size of the diameters ranging from $40 \mathrm{~nm}$ to 200 $\mathrm{nm}$, measurements of thermal conductivity may have some uncertainties.

\subsection{Ionic-liquid treatment on PEDOT:PSS for improved PF}

In 2007, the first study to determine the effects of ionic liquid (IL) treatment on the electrical conductivity of PEDOT:PSS was conducted by utilising five different ionic liquids. ${ }^{216}$ The study used, 1-butyl-3-methylimidazolium bromide (BMIM:Br), 
1- benzyl-3-methylimidazolium chloride (BzMIM:Cl), 1-butyl3-methylimidazolium tetrafluoroborate (BMIM:BF4), and 1-butyl-1-methylpyrrolidium chloride (BMPro:Cl) as well as 1 ethyl-3-methylimidazolium chloride (EMIM:CL). The BMIM:BF $\mathrm{BF}_{4}$ treated film achieved the highest conductivity of $136 \mathrm{~S} \mathrm{~cm}^{-1}$. More recently, significantly higher electrical conductivity has been shown by controlling molecular ordering in PEDOT:PSS chains by using 1-ethyl-3-methylimidazolium tetracyanoborate (EMIM:TCB) thus, achieving an electrical conductivity of $2103 \mathrm{~S}$ $\mathrm{cm}^{-1} .^{217}$ PEDOT:PSS-ionic liquid composites have also exhibited resistance to mechanical deformation while maintaining high performance. ${ }^{218}$ These properties of PEDOT:PSS - ionic liquid composites make them particularly suitable candidates for printing onto flexible substrates and textiles because they can withstand shape deformation that could occur in wearable electronics. ${ }^{218}$

\subsection{Improved PF in (OHTEGs) through nano-composition}

PEDOT:PSS based nanocomposites can be synthesized through various means. In some cases, a simple mixture of nanorods, nanowires or nanoplatelets to the polymer matrix with ultrasonication to improve homogeneity can yield effective nanocompositions with desired thermoelectric properties. ${ }^{219,220}$ Tin selenide has been shown to boast a record ZT of 2.6 due to its high Seebeck coefficient and low thermal conductivity along the b-axis. This can be obtained by controlling the crystal growth and the axis it's measured at. ${ }^{221-223}$ Although the ZT of SnSe is high it's electrical conductivity is low. It has been therefore realized if composited with a conducting polymer matrix and post treated with solvents or acids a higher electrical conductivity and Seebeck coefficient are possible. ${ }^{149}$ The desired stoichiometry of tin to selenium in tin selenide nanosheets has been achieved through ball milling and subsequent hydrothermal lithium intercalation. After mixing with PEDOT:PSS it was discovered that the Seebeck coefficient was directionally correlated with increased mass fraction of the inorganic nanosheet meanwhile the electrical conductivity decreased with increased nanosheet content. ${ }^{221}$ A further treatment with 5\% DMSO resulted in an optimised PF of $386 \mu \mathrm{W} \mathrm{m}{ }^{-1} \mathrm{~K}^{-2}$. 221 Although $(\kappa)$ wasn't decoupled from the PF since there was an evident rise from 0.25 to $0.6 \mathrm{~W} \mathrm{~m}^{-1} \mathrm{~K}^{-1}$ the study shows the potential to utilize inorganic nanosheets to significantly tune $(\sigma)$ and $(S)$. 
In a recent study, tellurium nanorods were created in a solvothermal vessel and mixed with PEDOT:PSS. The corresponding solution was drop casted and post treated with $12 \mathrm{M} \mathrm{H}_{2} \mathrm{SO}_{4}{ }^{219}$ The different concentration of tellurium nanorods to PEDOT:PSS gave different Seebeck coefficient because of the change in Fermi level which was dependent on higher tellurium content. Through the $12 \mathrm{M} \mathrm{H}_{2} \mathrm{SO}_{4}$ treatment on the $90 \%$ tellurium to PEDOT:PSS ratio the carrier concentration was increased to give an optimised trade-off between $(\sigma)$ and $(S)$ of $204 \mathrm{~S} \mathrm{~cm}^{-1}$ and $84 \mu \mathrm{V} \mathrm{K}^{-1}$ respectively to produce a PF of $141 \mu \mathrm{W} \mathrm{m} \mathrm{m}^{-1} \mathrm{~K}^{-2} \cdot{ }^{219}$

In other cases, the nano-composition may be reached by making PEDOTnanowires and telluride nanoparticles in the same reaction vessel where there are inorganic nanoparticles imbedded on PEDOT nanowires. ${ }^{224,225}$ Through polar solvent vapor annealing (PSVA) it's been shown that both $(\sigma)$ the $(\mathrm{S})$ can improve drastically by controlling the (PSVA) time. ${ }^{226,227}$ PEDOT:PSS/Bi $\mathrm{Be}_{3}$ nanowires were synthesized and exposed to DMSO vapor at different rates. At 120 minutes (PSVA) exposure, an optimum Seebeck coefficient of $45 \mu \mathrm{V} \mathrm{K}^{-1}$ and a conductivity of $1000 \mathrm{~S}$ $\mathrm{cm}^{-1}$ was achieved thereby leading to a power factor of $223 \mu \mathrm{W} \mathrm{m} \mathrm{K}^{-1}$. Effectively there was a decrease in the density of states near the Fermi level which lead to the improved Seebeck coefficient. ${ }^{228}$ It was realized that if the bismuth telluride nanowires had a higher work function than PEDOT:PSS then there would be a positive change in barrier energy which lead to confirmed higher Seebeck coefficient and if the inverse was true then a lower Seebeck coefficient would be observed. ${ }^{227}$ This study gives insight into the relative work function ratio between a polymer-inorganic matrix and its influence on the charge carrier mobility/density.

Some studies have utilized nanosheets instead of nanowires/nanorods. ${ }^{149,229,230}$ In a study tin-selenium-telluride films were synthesized in a hydrothermal reaction and intercalated with lithium ions. After mixing with PEDOT:PSS a small PF $\left(14 \mu \mathrm{W} \mathrm{m}{ }^{-1}\right.$ $\mathrm{K}^{-2}$ ) was observed owning a further post treatment wasn't employed to improve electrical conductivity. ${ }^{231}$ The relative percentage of tellurium nanosheets to PEDOT:PSS was directly related to the electrical conductivity and Seebeck coefficient, in which higher concentrations of tellurium would yield higher electrical conductivity and lower Seebeck coefficient. However nanosheet composites showed promise as TEDs since the devices made were exposed to 1000 bends yet resulted in minimal degradation of either $(\sigma)$ or $(\mathrm{S}) .{ }^{231}$ In a more recent paper, the same team 
managed to improve the PF to $130 \mu \mathrm{W} \mathrm{m}{ }^{-1} \mathrm{~K}^{-2}$ using a similar Tin-selenium-telluride system with PEDOT:PSS reported preiviously. ${ }^{230,231}$ The main difference between the two research methodologies was the stoichiometry of the inorganic ingot to be imbedded into PEDOT:PSS matrix. The Seebeck coefficient and electrical conductivity however remained coupled and expressed an inverse relationship to each other.

Except for nano composition through hydrothermal and inorganic blending with polymer, it's possible to also use electroplating techniques to deposit both the polymer and or the inorganic nano particle to form a composite. ${ }^{205,232,233}$

A PEDOT-tellurium nano composite was synthesized via an EDOT oxidative polymerisation using iron chloride $\left(\mathrm{FeCl}_{3}\right)$ and the subsequent electroplating of tellurium ions on the PEDOT thin film surface. A high PF of $240 \mu \mathrm{W} \mathrm{m}{ }^{-1} \mathrm{~K}^{-2}$ was achieved by varying electroplating time in which it was discovered that the $(\mathrm{S})$ improved linearly with time meanwhile $(\sigma)$ decreased but in response to time. The overall PF was higher because the improvement of the (S) outweighed the reduced $(\sigma)$. Although, the film was bent 400 times it maintained 93\% of initial electrical conductivity thus solidifying potential for repeated use as a flexible device. ${ }^{204}$

\subsection{N-type PEDOT:PSS thermoelectrics}

Due to the difficulty in obtaining stable n-type PEDOT:PSS semiconductors the research lags in comparison to the p-type semiconductor. ${ }^{141}$ Recent studies however have found several synthetic routes where carbon nanotubes can be imbedded within the PEDOT:PSS matrix. ${ }^{234}$ Following a subsequent treatment with certain electron donor molecules such as organic, inorganic bases, or primary amines n-type characteristics can be observed and deduced through the sign change of the Seebeck coefficient from positive (denoting p-type) to negative (denoting n-type).

Wang et al synthesized a PEDOT-CNT composite via the oxidative polymerisation of EDOT with $\mathrm{FeCl}_{3}{ }^{235}$ The resultant composite was p-type as expected, however after being exposed to the vapor of TDAE (Tetrakis, dimethyl amino, ethylene) the composite showed n-type behaviour. Although the composite showed positive relationship between CNT concentration and electrical conductivity since the CNTs used in the study had much higher conductivity than the polymer; after 
treatment with TDAE the relationship ceased and due to the reduction of charge carriers, a much smaller electrical conductivity was observed. Still, the PF derived was extremely high $\left(1050 \mu \mathrm{W} \mathrm{m} \mathrm{m}^{-1} \mathrm{~K}^{-2}\right)$ due to the significantly improved large negative Seebeck coefficient above $-2 \mathrm{mV} \mathrm{K}^{-1}{ }^{235}$

Along the same logic, Kim et al, synthesized PEDOT:PSS/CNT composites by a wet spinning method and formed conductive ribbons. ${ }^{234}$ After being exposed to less than $1 \%$ of the reducing agent (hydrazine) the films maintained p-type characteristics and a high PF of $83 \mu \mathrm{W} \mathrm{m}{ }^{-1} \mathrm{~K}^{-2}$ was obtained. Increased concentration of hydrazine above $1 \%$ and further exposure to amine rich polyethyleneimine (PEI) confirmed a sign change from $\mathrm{p}$ to $\mathrm{n}$ in which resulted in a high $\mathrm{PF}$ of $113 \mu \mathrm{W} \mathrm{m} \mathrm{K}^{-1}$. The Seebeck coefficient was insensitive to the concentration of CNT in the film however the electrical conductivity showed a positive relationship with concentration of CNT. ${ }^{234}$ Other studies have utilized metal ions to bind with the polymer in which the metal ion at the centre can act as an electron acceptor and through reduction tuned for n-type characteristics. In a recent study, $\mathrm{CuCl}_{2}$ was mixed with a solution of PEDOT:PSS and casted on (PET) Poly (ethylene terephthalate) substrate. ${ }^{141}$ The mixed ionic (anion/cation) doping induced not only a higher Seebeck coefficient which reached over $-18 \mathrm{mV} \mathrm{K}^{-1}$ but with the improved electrical conductivity due to free mobile $\mathrm{Cl}^{-}$ anions a PF of $1.7 \mu \mathrm{W} \mathrm{m}{ }^{-1} \mathrm{~K}^{-2}$ was realized. The n-type characteristic was directly dependent on the concentration of $\mathrm{CuCl}_{2}\left(\mathrm{Cu}^{2+}\right)$ in which over $15 \% \mathrm{CuCl}_{2}$ relative to the polymer resulted in a sign change. The electrical conductivity was linearly correlated with $\mathrm{CuCl}_{2}$ concentration. ${ }^{141}$

This is relevant when considering optimisation strategies to have $\mathrm{p}$ and $\mathrm{n}$ junctions. Having the same material at both legs would be easier and more cost effective for large area printing and manufacturing endeavours. Although it has been shown that n-type electron transport may be observed in PEDOT:PSS after either metal ion incorporation within polymer matrix or via imbedding CNTs and subsequent reducing agent treatment to change sign from $\mathrm{P}$ to $\mathrm{N}$, it is difficult to make PEDOT:PSS n-type without compositing with foreign nanoparticles. chapter 6 will explore a novel synthetic route to n-type SWNT-PEDOT:PSS composite with minimal CNT concentration through an extremely fast solvothermal reaction. 


\subsection{Graphene and CNT based PEDOT:PSS composites}

The semi-metallic allotrope graphene and semiconducting carbon nanotubes have intrinsically favourable electronic properties of interest in the fabrication and optimisation of thermoelectric, optoelectronic, piezo electronic and wearable electronic materials. ${ }^{236-239}$ Pure graphene thermoelectric devices have been fabricated whereby Seebeck coefficients of $90 \mu \mathrm{V} \mathrm{K}{ }^{-1}$ have been measured. ${ }^{240}$ Graphene (rGO)/fullerene $\left(\mathrm{C}_{60}\right)$ nanocomposites were subsequently prepared with PEDOT:PSS, and the corresponding results indicated that phonon scattering in the form of layered film deposition 50-1000 layers; by the introduction of grain boundaries slowed down the increase in thermal conductivity relative to the increasing electrical conductivity. ${ }^{241-244}$ The optimum power factor corresponded to the 3:7 ratio between $\left(\mathrm{C}_{60}\right)$ and $(\mathrm{rGO})$ respectively. It was found that increasing percentage composition of (rGO) increased electrical conductivity as well as thermal conductivity, meanwhile $\left(\mathrm{C}_{60}\right)$ increased the Seebeck coefficient while simultaneously decreasing electrical and thermal conductivity, thus revealing the potential for engineering favourably thermopowers in polymer composites when utilizing the distinct properties of graphene and fullerene. ${ }^{245}$ Due to the increase in thermal conductivity from 0.2 to $2 \mathrm{~W}$ $\mathrm{m}^{-1} \mathrm{~K}^{-1}$ and an optimised Seebeck coefficient of $21.8 \mu \mathrm{V} \mathrm{K} \mathrm{K}^{-1}$ the nanohybrid was optimised to give a ZT value of $6.7 \times 10^{-2} .^{246}$

In other work, graphene polymer composites were fabricated via in-situ polymerisation of dispersed graphene in the presence of PSS', and subsequent oxidative polymerisation in the presence of EDOT. The resultant thin films displayed electrical conductivity of $637 \mathrm{~S} \mathrm{~cm}^{-1}$ and a Seebeck coefficient of $26.78 \mu \mathrm{V} \mathrm{K}^{-1}$; thus leading to an optimised power factor of $45 \mu \mathrm{W} \mathrm{m} \mathrm{m}^{-1} \mathrm{~K}^{-2}$ which was shown to be $93 \%$ higher than that of the pristine PEDOT:PSS. ${ }^{247}$ The same research group also investigated hybrid structures of PEDOT:PSS/graphene/MWNT. The three-part composite had superior thermoelectric properties compared to the pristine 
PEDOT:PSS, PEDOT/graphene and PEDOT/MWNT composites. The electrical conductivity was $689 \mathrm{~S} \mathrm{~cm}^{-1}$, while the PF and Seebeck coefficient were $37.08 \mu \mathrm{W} \mathrm{m}{ }^{-}$ ${ }^{1} \mathrm{~K}^{-2}$ and $23.20 \mu \mathrm{V} \mathrm{K}^{-1}$ respectively. The $\mathrm{ZT}$ value increased from 0.017 for pristine PEDOT:PSS to 0.031 for the PEDOT:PSS/graphene/MWNT composites. ${ }^{248}$ The recent work on these ternary composites reports the effects of different weight ratio between PEDOT/graphene/carbon nanotube based ternary composite as well as the effects of acid treatment on the electrical conductivity and Seebeck coefficient. Electrical conductivities of $208.4 \mathrm{~S} \mathrm{~cm}^{-1}$ were reported with enhancement to power factors going from $1.96 \mu \mathrm{W} \mathrm{m}{ }^{-1} \mathrm{~K}^{-2}$ to $9.10 \mu \mathrm{W} \mathrm{m}{ }^{-1} \mathrm{~K}^{-2}$ upon acid treatment. ${ }^{249}$ These findings infer the potential for optimising the thermopower based on synergistic electronic effects between different intrinsic properties presented by the materials selected and device fabrication synthesis methods employed. ${ }^{245,247}$ Essentially PEDOT:PSS acts as a polymer matrix with low thermal conductivity for charge transport meanwhile the CNTs depending on size/diameter may introduce phonon scattering properties and higher Seebeck and electrical conductivity. ${ }^{250-252}$

\subsubsection{Carbon nanotubes}

Despite having relatively high thermal conductivity, carbon nanotube composites are the focus of much research due to their high electrical conductivity and ease of processing. PEDOT:PSS-carbon nanotubes and polyvinyl acetate composites have been synthesized. The report showed that thermopower was weakly correlated with the electrical conductivity, resulting in large thermoelectric power factors of 160 $\mu \mathrm{W} \mathrm{m}{ }^{-1} \mathrm{~K}^{-2} .{ }^{253}$ The highest room temperature electrical conductivity and Seebeck coefficient along the in-plane direction was shown to be $1350 \mathrm{~S} \mathrm{~cm}^{-1}$ and $41 \mu \mathrm{V} \mathrm{K}^{-1}$ respectively. Without additional DMSO secondary doping, the PEDOT:PSS grade PH1000 showed higher thermopowers while PH500 had better electrical conductivities. Carbon nanotube / poly(vinyl chloride) nanocomposites were fabricated by dispersing carbon nanotubes in an N-type poly(nickel 1,1,2,2ethenetetra-thiolate) (PETT). The conductive N-type polymer replaced PEDOT: PSS as a dispersant for CNTs. The optimized power factor for methanol treated PETTCNT-polyvinyl chloride was $58.6 \mu \mathrm{W} \mathrm{m}^{-1} \mathrm{~K}^{-2}$, while the electrical conductivity and Seebeck coefficient were, $629.9 \mathrm{~S} \mathrm{~cm}^{-1}$ and $30.5 \mu \mathrm{V} \mathrm{K}^{-1}$ respectively and demonstrated a high ZT value of $0.3 .^{254}$ 
Polystyrene has been utilised as a matrix for SWNTs to reduce thermal conductivity yielding an optimum performance at $20 \%$ by weight polystyrene, giving a power factor of $85 \mu \mathrm{W} \mathrm{m} \mathrm{m}^{-1} \mathrm{~K}^{-2}$ for the composite derived from a Seebeck coefficient of $57 \mu \mathrm{V} \mathrm{K}^{-1}$ and an electrical conductivity of around $285 \mathrm{~S} \mathrm{~cm}^{-1}$. Of note, the thermal conductivity decreased with the addition of polystyrene. The polymer composite offered a 1.7 times higher power factor than the pristine SWNT. ${ }^{255}$ Other work with SWNT shows an improved performance by a layered morphology in the form of SWNTs sandwiched by polypyrrole (PPy) nanowires. The composites showed a Seebeck coefficient of 41.3 $\mu \mathrm{V} \mathrm{K} \mathrm{K}^{-1}$, a conductivity of $802.6 \mathrm{~S} \mathrm{~cm}^{-1}$ and a maximum power factor of $21.7 \mu \mathrm{W} \mathrm{m}{ }^{-1} \mathrm{~K}^{-2}{ }^{256}$ The results signify the potential in small molecule and polymer thermoelectric device optimisation without relying on PEDOT:PSS.

In addition, some studies show that the Seebeck increases in semiconducting-SWNT (s-SWNT) with only a slight increase in thermal conductivity with increasing temperature due to the amorphous grain structure in bulk CNTs. ${ }^{250,257}$ The Studies conducted presented high Seebeck coefficients from different methods including treatment of metallic and s-SWNT with different acids. It appears from several studies that by filtering out metallic CNTs, high Seebeck coefficients may be induced by the s-SWNTs. ${ }^{250,258,259}$ Other articles describe the research potentials in filtering the different forms of nanotubes and implications of the diameter and size of the nanotubes in relations to thermal conductance, electrical conductance and optoelectronic properties. ${ }^{250,260,261}$ A high Seebeck of $170 \mu \mathrm{V} \mathrm{K}^{-1}$ was presented by the un-doped sSWNT but with a much lower PF owning to the low conductivity of the s-SWNT. The acid treatment may have decreased resistivity however the Seebeck decreased also

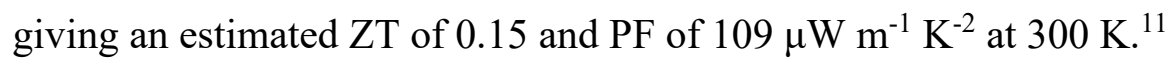

\subsubsection{Metal halide Perovskites oxides}

Lead halide perovskites have recently become a hot topic in the area of photovoltaics, ${ }^{262-264}$ and indeed it has been shown that the lead halide perovskites have high Seebeck $\left(\sim 10^{5} \mu \mathrm{V} \mathrm{K}^{-1}\right)$ at room temperature. ${ }^{265}$ Ab initio calculations suggest $\mathrm{ZT}$ values of 1-2 for the $\mathrm{CH}_{3} \mathrm{NH}_{3} \mathrm{PbI}_{3}$ and $\mathrm{CH}_{3} \mathrm{NH}_{3} \mathrm{SnI}_{3}$ perovskites, due to high carrier mobility, small carrier effective masses and weak electron-phonon and hole phonon couplings. ${ }^{266}$ Several other researchers have experimentally determined high Seebeck coefficients of $\mathrm{s}_{\mathrm{s}=\text {.dark }} 820 \mu \mathrm{V} \mathrm{K}^{-1}$ to $\mathrm{s}=$ light $540 \mu \mathrm{V} \mathrm{K} \mathrm{K}^{-1} \cdot{ }^{24,267}$ Due to the low electrical 
conductivity owning to a low carrier concentration, doping is necessary in order to increase conductivity. Research into making stable doped organo-metallic perovskites is needed. Interestingly the perovskite material exhibits different Seebeck coefficients depending on light environment due to charge availability upon photon excitation. The same papers conducted several experiments with doped $\mathrm{CH}_{3} \mathrm{NH}_{3} \mathrm{SnI}_{3}$ showing the potential for high ZT of 3 with the optimum electrical conductivity.

Other lanthanum cobalt oxide and titanium based type of perovskites have been synthesized and high Seebeck coefficient of $-200 \mu \mathrm{V} \mathrm{K}^{-1}$ have been obtained by controlling the spin state of the cations and a sign change from positive to negative with $\mathrm{Ti}^{4+}$ doping. ${ }^{268}$ Other higher (S) are reported in the article ranging from -200 to $450 \mu \mathrm{V} \mathrm{K}^{-1}$ depending on the doping type and concentrations. Research into increasing the electrical conductivity of these materials without adversely affecting their low thermal conductance would prove beneficial to both thermoelectric, photovoltaic and thermo-photovoltaics. ${ }^{269-272}$ Table 2.7.2.1 depicts the thermoelectric properties of material discussed in chapter 2.7. In a paper by Fenwck et al. ${ }^{273}$ a high ZT of 0.14 was obtained from a caesium tin iodide $\left(\mathrm{CsSnI}_{3}-{ }_{\mathrm{x}} \mathrm{Cl}_{\mathrm{x}}\right)$ perovskite at chlorine doping concentration of $1 \%$. The high ZT was in part due to a high electrical conductivity due to the self-doping mechanism through the oxidation state change from $\mathrm{Sn}^{+2}$ to $\mathrm{Sn}^{+4}$ thereby introducing holes in the system. The seebeck coefficient showed a positive relationship with increasing temperature meanwhile the thermal conductivity decreased at higher temperatures.

Table 2.7.2.1 depicting the thermoelectric properties of materials discussed in chapter 2.7

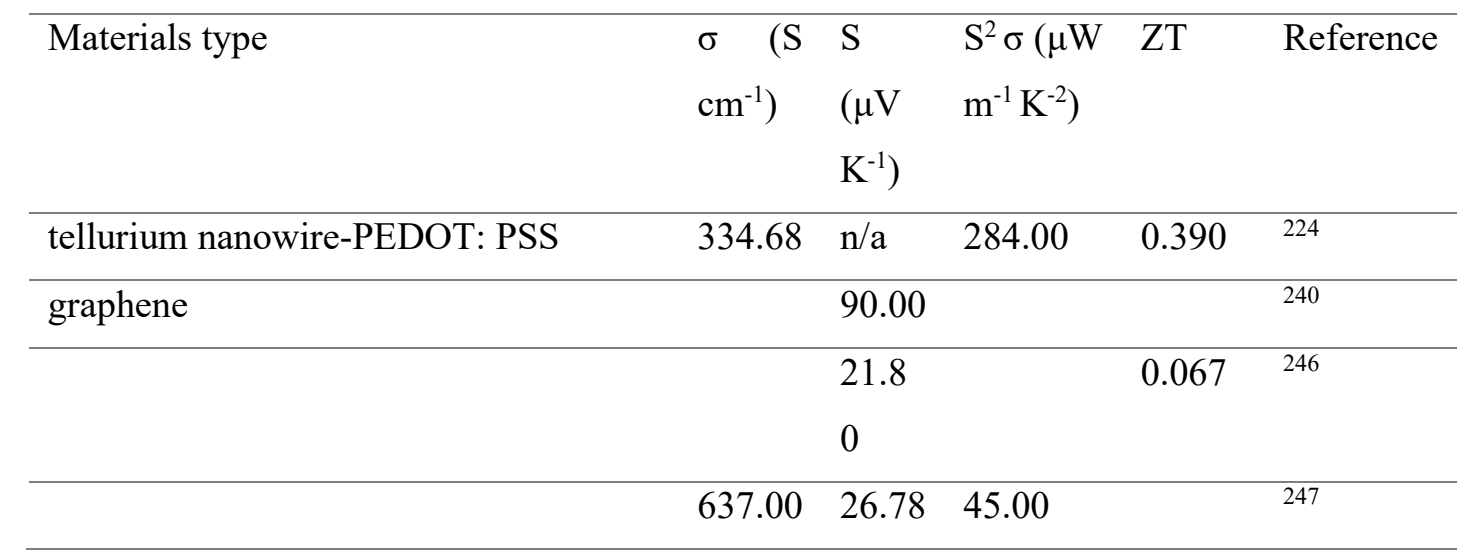




\begin{tabular}{|c|c|c|c|c|c|}
\hline PEDOT/graphene and PEDOT/ MWNT & 689.00 & 23.20 & 37.08 & 0.031 & 248 \\
\hline PEDOT/graphene and PEDOT/ CNTs & 208.40 & & 9.10 & & 249 \\
\hline \multirow[t]{2}{*}{ PEDOT:PSS-graphene- $\mathrm{TiO}_{2}$} & 260.00 & - & 47.00 & 0.0048 & 274 \\
\hline & & 47.00 & & & \\
\hline Carbon nanotubes & 1350.0 & 41.00 & 160.0 & & 253 \\
\hline poly(vinyl chloride)-CNTs & 629.90 & 30.50 & 58.60 & 0.30 & 254 \\
\hline Polystyrene-SWNT & 285.00 & 57.00 & 85.00 & & 255 \\
\hline polypyrrole (PPy)-SWNT & 802.60 & 41.30 & 21.70 & & 256 \\
\hline Pure S-SWNT & & 170.0 & & & 250 \\
\hline Acid treated-S-SWNT & & & 108.6 & 0.15 & 250 \\
\hline perovskites $_{\mathrm{s}=\text {.dark }}$ & & 820.0 & & & 266 \\
\hline Perovskite $=$ light & & 540.0 & & & 266 \\
\hline Lanthanum oxide perovskites & & -200 & & & 268 \\
\hline Doped lanthanum perovskites & & 450 & & & \\
\hline $\mathrm{CsSnI} 3-\mathrm{xCl} x$ & $<100$ & $<140$ & & 0.14 & 273 \\
\hline
\end{tabular}

\subsection{Strategies to decouple (S) from $(\sigma)$ in PEDOT:PSS}

It's been shown that the electrical conductivity, Seebeck coefficient and thermal conductivity can be altered by simple solvent treatment or nano composition with inorganic nano material with desired properties to optimize PF and ZT. ${ }^{149,219,227,229,275}$ It appears that methods that consider the trade off and discover a way to offset them towards higher PF would yield higher ZT. A recent study by, Xu et $a l$ in which a triple post treatment was conducted on PEDOT:PSS to yield a high PF of $141 \mu \mathrm{W} \mathrm{m}{ }^{-1} \mathrm{~K}^{-2}$ at room temperature. ${ }^{276}$ This was due to the fine tuning of oxidation level through the reducing agent $\left(\mathrm{NaBH}_{4}\right)$; which improved the Seebeck coefficient to $28 \mu \mathrm{V} \mathrm{K}^{-1}$ and treatments with formamide $\left(\mathrm{CH}_{3} \mathrm{NO}\right)$ and sulfuric acid $\left(\mathrm{H}_{2} \mathrm{SO}_{4}\right)$ which improved electrical conductivity to $1786 \mathrm{~S} \mathrm{~cm}^{-1} .{ }^{276}$ The study confirms that it's possible to obtain high PF without using rare earth minerals to improve Seebeck coefficient however the study didn't investigate long term stability of the Seebeck coefficient. This concern stems from the fact that the Seebeck coefficient derived from oxidation level changes (reduction) is unstable due to tendencies to (re oxidise) in air 
without counter ions to stabilize the increased electron density that has been induced into the film since the reducing agent tends to also remove counter ions (PSS').

In a study by Lee et al a PEDOT:PSS/CNT composite was post treated with ethylene glycol to yield a high PF of $151 \mu \mathrm{W} \mathrm{m} \mathrm{m}^{-1} \mathrm{~K}^{-2}$. The performance of the composite derived from intrinsically the high conductivity of the carbon nanotube and the selective removal of PSS induced by the ethylene glycol treatment which further improved the electrical conductivity without significant reduction in the Seebeck coefficient for the $20 \%$ CNT to PEDOT:PSS composite. ${ }^{145}$. Due to DMSO not affecting the carrier concentration the Seebeck coefficient is not significantly affected by the DMSO doping stage and thereby explaining the simultaneous improvement to Seebeck coefficient and electrical conductivity. In a paper by Liu et al. ${ }^{281}$ An explanation for the origin of the simultaneous improvement to the Seebeck coefficient and the electrical conductivity for some PEDOT:PSS-CNT composites was proposed. The first is that an increase in the Seebeck coefficient of the composite as more CNT is added is observed. The higher CNT loading also increased the electrical conductivity slightly. The second mechanism is due to the electrical conductivity increasing in response to DMSO treatment. This can be explained by a weakened coulombic attraction between PEDOT and PSS thereby making PEDOT chains more linear as well due to the presence of CNT forming an extended network of PEDOT-CNT with better carrier mobility is established.

In a more recent study by Liu et al one of the highest PF for an OHTEG was achieved by incorporating single walled carbon nanotube (SWNT) within PEDOT:PSS. As the SWNT were added to the polymer matrix up to $50 \% \mathrm{w} / \mathrm{w}$, both $(\mathrm{S})$ from $(\sigma)$ increased and a power factor of $190 \mu \mathrm{W} \mathrm{m}{ }^{-1} \mathrm{~K}^{-2}$ was obtained. ${ }^{71}$ Another set of films were prepared with DMSO. There was a further increase in the electrical conductivity however the Seebeck coefficient although increased with carbon nanotube content was not affected by DMSO treatment. This led to a PF of $270 \mu \mathrm{W} \mathrm{m}{ }^{-1} \mathrm{~K}^{-2}$. The optimised film with DMSO treatment and a further $0.1 \mathrm{M} \mathrm{NaOH}$ post treatment led to a high PF of $526 \mu \mathrm{W} \mathrm{m}{ }^{-1} \mathrm{~K}^{-2} .{ }^{71}$ The electrical conductivity as expected did reduce slightly due to lower carrier concentration as determined by hall effect measurements but the optimised Seebeck coefficient outweighed the trade-off. The issue however was that the films re oxidised after 3 days. The film regained some electrical conductivity from the base treatment however the Seebeck coefficient was drastically reduced. Therefore, 
strategies to improve the Seebeck coefficient without re-oxidation by using stabilizing ions such as ionic liquids or finding a synthetic route that does not change the oxidation state would yield more commercially viable films. 


\section{Chapter 3 Materials and Methods}

This chapter will focus on presenting relevant materials and methods used in this research study. The first section will focus on providing the type of material used as well as where it was obtained. The rest of the chapter will focus on giving more detailed explanation on the analytical tools and techniques used to either prepare samples and or measure thermoelectric properties of the samples.

\subsection{Materials}

All chemical reagents used were purchased from (Sigma-Aldrich, Gillingham, England, United Kingdom), unless stated otherwise. PEDOT:PSS Heraeus Clevios PH1000 is the main matrix used for this research. Unless stated otherwise, all chapters will use this material where stated PEDOT:PSS. Other materials used in all the chapters in this research include, water, isopropyl alcohol, (IPA), acetone, and methanol for glass cleaning and nitrogen gas for drying glass.

\subsubsection{List of chemicals used in Chapter 4 (Effects of solvent treatment on PEDOT:PSS thin films)}

1. PEDOT:PSS Heraeus Clevios $\mathrm{PH} 1000$ (1.3\% solid content in $\mathrm{H}_{2} \mathrm{O}$ )

2. 1-4-Dioxane (anhydrous, 99.8\%)

3. 1-Methyl-2-propanone (anhydrous, 99.5\%)

4. 1-Methy-2-pyridone ( anhydrous, 99.5\%)

5. (DMSO) Dimethyl sulfoxide (anhydrous,99.9\%)

6. (EG) Ethylene glycol (anhydrous, 99.8\%)

7. 4-Hydroxy-4-methyl-2-pentanone (99\%) 
8. Propylene glycol $(99.5 \%)$

9. Propylene carbonate ( anhydrous, 99.7\%)

\subsubsection{List of chemicals used in chapter 5 (Enhanced Electrical Conductivity and Seebeck Coefficient in PEDOT:PSS via a Two-Step Ionic liquid and $\mathrm{NaBH}_{4}$ Treatment)}

1. $\left(\mathrm{NaBH}_{4}\right)$ Sodium borohydride powder $(99 \%$ Reagent grade $)$

2. (EMIM:TFSI)1-Ethyl-3-methylimidazoliumbis(trifluoromethylsulfonyl)imide (ionic liquid 98\% HPLC grade)

3. (DMSO) Dimethyl sulfoxide (anhydrous liquid 99.9\%)

\subsubsection{List of chemicals used in chapter 6 (Synthesis of N type SWNT- PEDOT:PSS composites)}

1. Tetrabutyle ammonium hydroxide $(\mathrm{TBAOH})$

2. Single walled carbon nanotubes $(0.5 \%)$ suspended in $95 \%$ isopropyl alcohol (SWNT) (mixed metallic/semiconducting)

3. Dimethyl sulfoxide (DMSO) anhydrous liquid $99.9 \%$

\subsubsection{List of chemicals used in chapter 7 (Towards decoupled Seebeck coefficient from Electrical conductivity)}

1. Single walled carbon nanotubes $(0.5 \%)$ suspended in $(95 \%)$ isopropyl alcohol

2. 1-Ethyl-3-methylimidazolium bis(trifluoromethyl sulfonyl)imide EMIM:TFSI

3. (GuI) Guanidinium Iodide (99\%, anhydrous)

4. (EG) Ethylene glycol (anhydrous, 99.8\%) 


\subsection{Methods}

This section describes the generalized materials and methods processing that encapsulates all chapters. More detailed (deviations or additions to this section) chapter specific methods will be included in each chapter as appropriate.

\subsubsection{Fabrication of PEDOT:PSS based thin films}

A $1 \mathrm{~mm}$ thick plain nonconductive glass is cut into $2.2 \mathrm{~cm}$ by $2.2 \mathrm{~cm}$ pieces and washed in a Helmanax solution under sonication in a $60{ }^{\circ} \mathrm{C}$ bath. The glass substrates are then rinsed with deionized water 3 times and subsequently rinsed with acetone and then isopropanol 3 times each. The glass is then dried in a stream of Nitrogen gas. The appropriate PEDOT:PSS dispersion/ composite solution is placed into the appropriate $7 \mu \mathrm{L}$ vials. Desired aliquots of dopant are added into the appropriate vial to give desired doping level. For example, to get a $2 \% \mathrm{v} / \mathrm{v}$ doping of a solvent, $60 \mu \mathrm{L}$ of desired solvent is added into a glass vial with $3000 \mu \mathrm{L}$ of PEDOT:PSS and mixed vigorously using a mechanical vortex mixer and then heated for a (time) to aid homogeneity and dispersion. $256 \mu \mathrm{L}$ (subject to slight deviations based on sample) of the PEDOT:PSS/solvent solutions are then pipetted onto a precleaned glass substrate and spin-coated at $2000 \mathrm{rpm}$ with an acceleration speed of 2000 $\mathrm{rpm} \cdot \mathrm{s}^{-1}$ for $30 \mathrm{~s}$, then annealed at $120{ }^{\circ} \mathrm{C}$ in air for $20 \mathrm{~min}$. The films produced will range typically between $50-70 \mathrm{~nm}$, as determined by stylus profilometry.

\subsection{Instrumental analysis}

\subsubsection{Dektak surface profiler}

The film thickness for each sample utilized in this study whereby it was used to determine the electrical conductivity was determined by using surface profilometry. ${ }^{277278}$ The technique measures the change in topography by maintaining the stylus tip on the sample at a given Force (mg). In this study it was established that a Force of around 1-3 mg was enough. The stylus moves in a fixed direction across the sample whereby it measures the difference in height (hills or valleys) between the 
regions it passes across. ${ }^{279279}$ To determine thicknesses in this research project a needle was used to scratch a valley into the sample whereby when the stylus passes through and the difference in height between the valley (hills) and the surface of the film is taken to determine film thickness. At least 5 different regions were measured for each sample and averaged out. In the case of films that were highly inhomogeneous different films were synthesized and measured.

\subsubsection{UV-Vis-NIR spectroscopy}

Ultraviolet-Visible (UV-Vis), Near-infrared (NIR) spectroscopy is a useful technique for studying the optical properties organic, organometallic and other species with excitable electrons. ${ }^{280,282}$ UV-Vis-NIR involves absorption spectroscopy and reflectance spectroscopy in the UV-Vis-NIR spectral region, whereby molecules containing $\pi$-electrons or non-bonding electrons are identified by their excitation to higher anti-bonding molecular orbitals via absorption of ultraviolet or visible light energy. ${ }^{283}$ For all experimental chapter except for chapter 6, UV-Vis-NIR spectroscopy was utilized to indicate the change in concentrations of charge carriers (qualitatively) however quantitative analysis can be utilized when utilizing BeerLambert law as indicated below in the equation.:

$$
A=\log _{10}\left(I_{\lambda \max } / I\right)=\epsilon_{\lambda \max } c L,
$$

where $A$ is the measured absorbance, $I_{\lambda \max }$ is the intensity of the incident light at the wavelength of maximum absorption $\left(\lambda_{\max }\right), I$ is the transmitted intensity, $L$ is the path length through the sample measured in centimeters, $c$ is the concentration $\left(\mathrm{molL}^{-1}\right)$ of the absorbing species, and $\epsilon_{\lambda \max }$ is a constant known as the molar absorptivity $\left(\mathrm{Lmol}^{-1} \mathrm{~cm}^{-1}\right)$ for each species at $\lambda_{\max }{ }^{284}$ According to the Beer-Lambert law, the concentration of an analyte can be calculated when $\epsilon_{\lambda \max }$ is known, $L$ is fixed, and $I_{\lambda \max }$ and $I$ are measured. ${ }^{285,286}$

PEDOT:PSS contains thiophene molecules at different oxidation states. The excitation of the oxidized or reduced species absorb light at a specific wavelength. Utilizing this technique (UV-Vis-NIR), it was possible to indicate the distribution of charge carriers via the analysis of the absorption intensity, whereby for the bipolarons it is $1800 \mathrm{~nm}$, polarons $900 \mathrm{~nm}$ and the $\pi-\pi^{*}$ is $600 \mathrm{~nm} \cdot{ }^{287,288}$ The UV-Vis-NIR absorbance spectra were measured with a Perkin Elmer Lambda 750 
spectrophotometer, using an uncoated $1 \mathrm{~mm}$ thick glass substrate as a reference sample and typically ran from $300 \mathrm{~nm}$ to $2400 \mathrm{~nm}$. Since all films used in this study did not exceed $100 \mathrm{~nm}$ the transmittance was used to analyze the films. A preliminary study was conducted by measuring the $\%$ transmittance to determine the penetration depth of UV-VIS-NIR on the PEDOT:PSS films. The data is represented in absorbance as shown in Figure 3.3.2. The Figure represents PEDOT:PSS at various thicknesses. At a thickness below $640 \mathrm{~nm}$ the absorbance is within an acceptable range $<1$ however above $640 \mathrm{~nm}$ and specifically at $1000 \mathrm{~nm}$ the spectrum is significantly above the penetration depth of the UV-VIS-NIR as shown by the noise and inconsistent spectrum. This suggests that above $640 \mathrm{~nm}$ the light is not able to penetrate the film properly. Films with higher thicknesses above that range would require UV-VIS-NIR measurement via \% reflectance instead. As also observed in literature films around $100 \mathrm{~nm}$ can reliably have their UV-VIS-NIR measured in transmittance. ${ }^{289}$ For comparison between literature studies as well as different films and chapters in this thesis all films were $<$ than $100 \mathrm{~nm}^{290}$

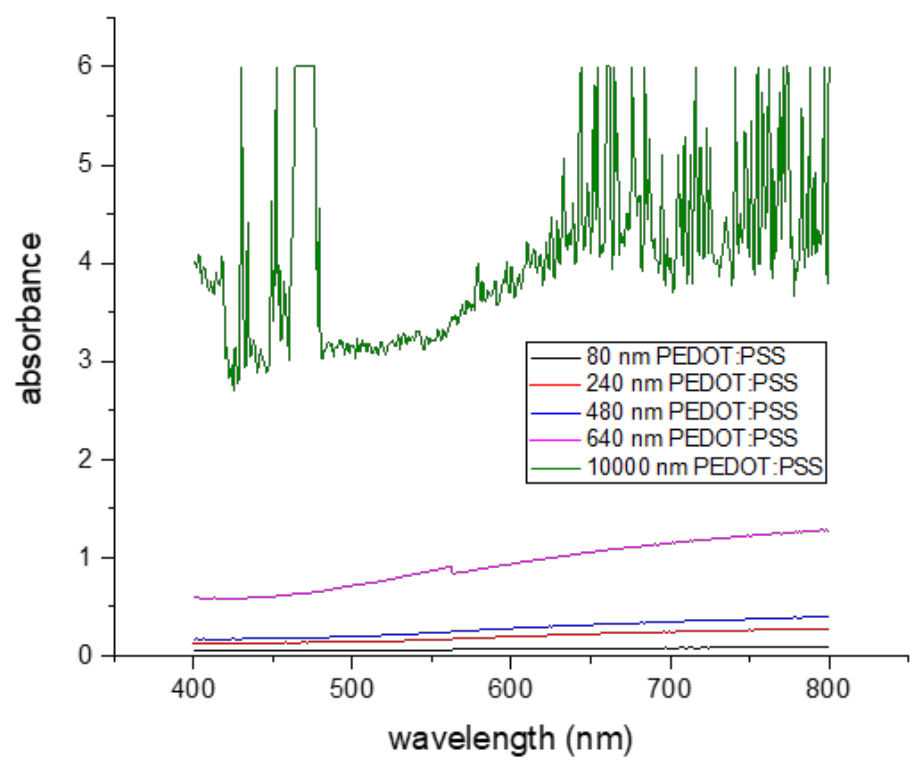

Figure 3.3.3, depicting the UV-VIS-NIR spectra of PEDOT:PSS films at various thicknesses represented in absorbance. 


\subsubsection{Raman spectroscopy}

Raman spectroscopy is a spectroscopic technique that functions on the principle of Raman scattering, in which monochromatic laser light is used to study the rotational, vibrational and other modes of a material . 291,292 Typically, Raman scattering occurs when an incident photon leads to the creation or annihilation of a phonon, and the interaction between the phonon and the laser light results in a shift in energy, or a degree of wavelength shift. ${ }^{293}$ This shift is exactly related to the resonant vibrational frequencies of the molecular bonds in the material being studied and provides the necessary information about the different modes of the material. ${ }^{294}$ Materials that cause such a shift in wavelength are referred to as Raman active materials, and PEDOT:PSS is an example of such a material. In all the experimental chapters in this research project Raman spectroscopy was utilized to study the sample composition and chemical shifts based on Raman active vibrational modes. This was useful for studying n-type doping of single walled carbon nanotubes (SWNTs) and SWNT-PEDOT:PSS samples due to the red shift at the G band. ${ }^{295-297}$ It was also possible to determine reduction of thiophene molecules through the spectral intensity and width shortening due to reduction at specific regions. The most significant utilization of this instrumental technique was in determining the Raman red shift at $1440 \mathrm{~cm}^{-1}$ which indicates the benzoid to quinoid conformational change in PEDOT chains. ${ }^{298-300}$ The spectra were run from $300 \mathrm{~nm}$ to $1900 \mathrm{~nm}$. The Raman spectra were measured using a Renishaw inVia Raman system. A $532 \mathrm{~nm}$ laser and a 50× objective lens was used to measure the Raman spectra of the samples with an acquisition time of $10 \mathrm{~s}$. Several repeats were conducted to make sure the Raman shifts were consistent and not due to surface inhomogeneity and spectra shown in this study were results of Raman shifts that were consistently observed.

\subsubsection{Xray photoelectron spectroscopy}

X-ray photoelectron spectroscopy (XPS) is a surface-sensitive qualitative and quantitative spectroscopic technique that measures the elemental composition, chemical states (via chemical shift), and electronic states of most elements. ${ }^{301302}$ Until recently, it was believed by many that hydrogen and Helium XPS spectra could not be obtained due to low Xray photoionization cross-sections pertaining to the 1s orbitals 
of hydrogen and Helium. ${ }^{303}$ A study by Hoffmann et al claimed with evidence that If a bright enough Xray source is used, then it is possible to study the elements. ${ }^{304}$ XPS is based on the photoelectric effect, where energy of ejected photoelectrons are measured after subjecting a material to X-ray bombardment. The kinetic energy measured during X-ray bombardment accurately identifies the chemical elements present within a material, since each element has a characteristic binding energy associated with its electron levels. Formally, the binding energy $(B E)$ of the photoelectron is calculated by the relationship:

$$
K E=h v-B E,
$$

where $h v$ is the incoming energy of the incident $\mathrm{X}$-ray photon and $B E$ is dependent on the chemical environment of the element, for instance, carbon in $\mathrm{C}_{2} \mathrm{H}_{6}$ has a different binding energy than carbon in $\mathrm{CH}_{4}$. Sulphur in $\mathrm{SH}_{2}$ and $\mathrm{SO}_{3}$ occupy different oxidation states thereby their BE would be different. ${ }^{305}$ The different bonding states will thereby give different binding energies in XPS spectrum obtained. For a given elemental study the position of the binding energy usually referred to as chemical shift indicates the oxidation state. For example, $\mathrm{SH}_{2}$ may have a binding energy at $162.5 \mathrm{eV}$ meanwhile the $\mathrm{SO}_{3}$ may have a binding energy at $168 \mathrm{eV}$ due to the oxidation state of the Sulphur being different in different bonding situations (chemical environments). 198,306,307 Therefore, XPS this is a great technique for studying molecular changes at the surface of materials. As observed for all experimental chapters, this technique was utilized to measure the chemical shifts of Sulphur $2 p$ peaks in order to study the reduction of thiophene carriers whereby a shift towards lower binding energy infers reduced carrier concentration. ${ }^{308}$ This was instrumental in also determining the oxidation state of other species such as Nitrogen via the position of the BE for Nitrogen 1s. ${ }^{295}$ For the peak fitting the software freely available CASA XPS (http://www.casaxps.com/) was used to model the high resolution XPS data. Theoretical chemical composition as well as specific known information about samples were used to create peak fitting models such as knowledge that Sulphur $2 p$ has Spin-orbital doublet whereby the 3/2 peak is twice as intense as the $1 / 2$ peak with a separation energy of 1.2. The paper by Chen et $a l^{309}$, Crispin et al ${ }^{196}$ and Salanec et $a l^{310}$ were the main papers used to help construct peak fitting models used in this research project. Multiple high-resolution peaks were taken at different locations and peaks shown in this study consisted of peaks that had 
consistent chemical shifts. XPS was carried out using a Kratos Axis Supra (Kratos Analytical), (Kratos Analytical Ltd, Manchester, United Kingdom), using a monochromated $\mathrm{Al} \mathrm{K \alpha}$ source.

\subsubsection{Thermoelectric characterization}

For the thermoelectric characterization, a commercial instrument (ULVAC ZEM-3) was utilized to conduct all electrical conductivity as well as Seebeck coefficient measurements. The basic equation that determines the measurement of the Seebeck coefficient is presented below. ${ }^{311}$

$$
S=\frac{-\Delta \mathrm{V}}{\Delta \mathrm{T}}
$$

The change in voltage $(\Delta \mathrm{V})$ across a sample under a fixed temperature gradient $(\Delta \mathrm{T})$ at a certain temperature $\mathrm{T}$ is expressed. The $\Delta \mathrm{T}=\mathrm{T}_{\text {Hot }}-\mathrm{T}_{\text {Cold. }}$ The voltage and Temperature are measured at the same position on the sample (please see Figure $3.3 .5 .1) .{ }^{312}$ 


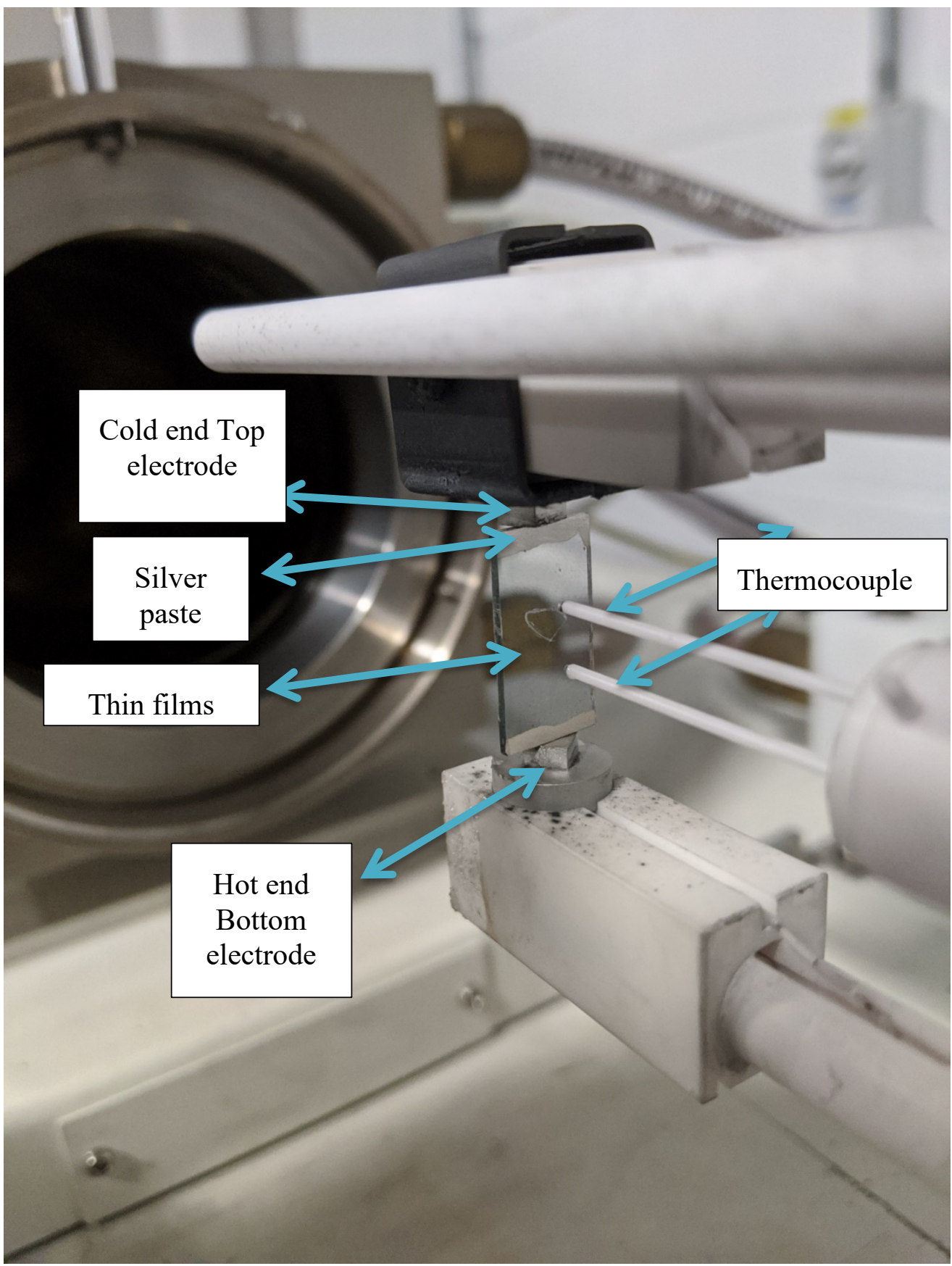

Figure 3.5.5.1 ZEM-3 set up showing electrodes and film measurement for Seebeck coefficient and electrical conductivity.

During the measurement of the Seebeck coefficient, the sample is affixed to 4 points of electrical contacts. This is achieved by forming electrical contact between the sample and the 4 electrodes via silver conductive paint across the glass component of the sample as well as forming a slight contact with the edge of the sample. The 
bottom electrode and top electrode are set at different temperatures as shown in Figure 3.5.5.1. a volt current (V/I) plot is generated to confirm adequate ohmic contact between sample and probes. If a straight line with an $\mathrm{R}^{2}$ value of 1 or extremely close is observed is observed, then it is assumed until further indications of error that the material has good contact with electrodes. Because measuring the Seebeck coefficient from a single point will undoubtably produce error. When determining the Seebeck coefficient the $\Delta \mathrm{T}$ is measured at three different $\Delta \mathrm{T}$ whereby $\left(\Delta \mathrm{T}=10,20\right.$ and $30{ }^{\circ} \mathrm{C}$ ) at each base temperature. For example, measuring the Seebeck coefficient of pristine PEDOT:PSS at $40{ }^{\circ} \mathrm{C}$ (base temperature) the ( $\Delta \mathrm{T}$ is measured at $\Delta$ of 10,20 and 30 ${ }^{\circ} \mathrm{C}$. To ensure the measurements are devoid of errors, a straight line between $\Delta \mathrm{V}$ against $\Delta \mathrm{T}$ at the 3 measurements as mentioned above is plotted whereby an $\mathrm{R}^{2}$ value close very close to 1 shows good measurement. The measurement is then allowed to measure at each successive base temperature and a further repeat is conducted for some samples to ensure the result is reliable. The Seebeck coefficient is taken from the slope of the plot and a further wire Seebeck deduction allows for determination of the Seebeck coefficient in volts at which it's converted to the units unutilized in this study $\left(\mu \mathrm{V} \mathrm{K}^{-1}\right) .{ }^{313}$ The electrical conductivity is derived from the sheet resistivity the ZEM3 system measures. Initially the film thickness in $(\mathrm{cm})$ is entered into the system pertaining to the sample. The sheet resistivity is measured at each of the three $\Delta \mathrm{T}$ at each base $\mathrm{T}$, and all data is checked for consistency to ensure sample is conductive with appropriate ohmic contact to ensure values obtained are reliable.

The sheet resistivity is obtained in $\Omega \mathrm{cm}$. The equation below.:

$$
\rho=\frac{1}{\sigma}
$$

is used to calculate the conductivity whereby $\rho$ is representative the resistivity $(\Omega \mathrm{cm})$ and $\sigma$ denotes the conductivity measured in siemens, $(1 / \Omega)$ in which for this research project it's denoted as $\left(\mathrm{S} \mathrm{cm}^{-1}\right) .{ }^{314}$ The equation below was used to determine the Power factor at each temperature, 


$$
P F=S^{2} \sigma
$$

Whereby S denotes the Seebeck coefficient and $\sigma$ the electrical conductivity. ${ }^{315,316}$

Within the research project a temperature range between $35^{\circ} \mathrm{C}$ to around $200{ }^{\circ} \mathrm{C}$ was used for the thermoelectric measurements. ULVAC ZEM-3 has the potential to measure between -130 to $1000^{\circ} \mathrm{C} .^{317}$ To measure thermoelectric properties below room temperature the cryogenic attachment is required. ${ }^{318}$ For measurements between room temperature and $1000{ }^{\circ} \mathrm{C}$ the Infrared furnace attachment is required. The justification for the measurement range in this study is due to the thermal stability of PEDOT:PSS being below $200^{\circ} \mathrm{C}$ as well as most research papers conducting measurements at room temperature or above. ${ }^{319,320}$ This allows for ease of comparison between literature and the data obtained in this study.

In relation to errors, the Seebeck coefficient were measured at 3 different $\Delta \mathrm{Ts}$ and the $\mathrm{R}^{2}$ value analysed. If the $\mathrm{R}^{2}$ value was less than 0.95 then it would indicate some issues with measurement. If it is less than 0.92 then it would indicate several repeats to be required. In terms of the films at least 3 repeats for each film where possible were made and averaged out to produce the results. For the solvents chapter the films were generally more difficult to measure than other chapters. The measurement limit for ULVAC-ZEM-3 is $100 \mathrm{k} \Omega$. Films below this limit were difficult to measure and any results obtained were discarded and experiments altered to yield more conductive films. 


\section{Chapter 4 Effects of solvent treatment on PEDOT:PSS}

\subsection{Introduction}

One of the most successful methods to dope PEDOT:PSS involves utilizing a series of polar solvents. ${ }^{196}$ (please refer to the definition regarding secondary dopants introduced in chapter 2.2; defined as introducing a chemical species that doesn't necessarily alter the oxidation state of PEDOT:PSS. ${ }^{187,321}$ The idea entails using solvents with a high dielectric constant and boiling point such as dimethyl sulfoxide (DMSO), ethylene glycol (EG) or their derivatives. ${ }^{322}$ Regarding the use of high boiling point solvents, the idea entails that most of the liquid would remain on the film surface long enough to act on the polymer instead of evaporating immediately before effecting morphological changes that may affect electrical conductivity. The improved electrical conductivity via solvent treatment involves polar or fully ionic solvents interacting with the oxygen group on the $\mathrm{PSS}^{-}$component in a way that leads to reduced coulombic interactions between the $\mathrm{PSS}^{-}$and $\mathrm{PEDOT}^{+}$thereby leading to the selective PSS removal from the film. ${ }^{270,323}$

This is even more important when utilizing a solvo-thermal treatment method (as will be shown later in chapter 7) whereby heat is used in conjunction with the ethylene glycol-guanidinium iodide solution to improve solvent penetration on the film. Since the discovery that PEDOT:PSS can conduct electricity, many researchers have published studies showing the effects of several different solvents to aid in improving the conductivity. Thus far a series of solvents of which include, ionic liquids, acids, and salt solutions of various solvents have been published in aim of improving electrical conductivity of PEDOT:PSS. ${ }^{316,324-326}$ The studies have incurred success in yielding significant improvement in the electrical conductivity of PEDOT:PSS from as low as $<1 \mathrm{~S} \mathrm{~cm}^{-1}$ to $>800 \mathrm{~S} \mathrm{~cm}^{-1}$. $210,299,327$ 


\subsection{Objective and overview}

This chapter will expand on studying the effects of internal solvent treatments with PEDOT:PSS solutions. Thin films of the solution will be synthesized and the electrical conductivity and Seebeck coefficient measured via ZEM-3 thermoelectric system. Solvents that have not been published or extensively studied in literature will be explored. ethylene glycol, and dimethyl sulfoxide will be used as they are well studied in the literature, thus will provide a comparable baseline to other solvents studied in this chapter. ${ }^{328}$ The effects of these solvents will also be studied using spectroscopic techniques such as Raman, XPS and UV-Vis-NIR to obtain mechanisms behind the effects. ${ }^{219,321}$

\subsection{Materials and methods}

For a generalized materials preparation of thin films in this study (please refer to chapter 3.2.1). To maintain literature comparability the films used in this study were formed from $5 \% \mathrm{v} / \mathrm{v}$ solutions of appropriate solvent to PEDOT:PSS PH1000 solutions.

\subsection{Materials processing}

To determine the optimal materials processing conditions many tests were conducted. A series of preliminary experimentation involving many repeats for solvent treated films under different spin conditions preceded the standards used for this research project. For this chapter, the spin speed was set as $2000 \mathrm{rpm}$, and the acceleration as $2000 \mathrm{rpm} \mathrm{s}^{-1}$ for 30 seconds because the conditions yielded the most homogenous films. It was discovered that by changing the spin speed, films with different thicknesses of the same stock solution could be produced. Below $1700 \mathrm{rpm}$, however, the film coverage of the $2.2 \mathrm{~cm}^{2}$ films was inconsistent, leading to poor quality films. The $2000 \mathrm{rpm}$ and $2000 \mathrm{rpm} \mathrm{s}^{-1}$ resulted in films with thicknesses ranging between 50 and $70 \mathrm{~nm}$. The viscosity as well as amount of solution pipetted, also affected the film thickness. The temperature used to anneal these films was set at $120^{\circ} \mathrm{C}$ for 20 minutes. As expressed in Figure 4.4.1, thermogravimetric analysis was 
used to confirm the choice of annealing temperature. The weight percentage of water was reduced from 100 to $2 \%$ within 15 minutes at $100{ }^{\circ} \mathrm{C}$. This is to be expected because water is the main solvent comprising in PEDOT:PSS solution since it is the solvent used to disperse the conductive polymer in which the manufacturer reports $<1.5 \%$ solid PEDOT:PSS content.

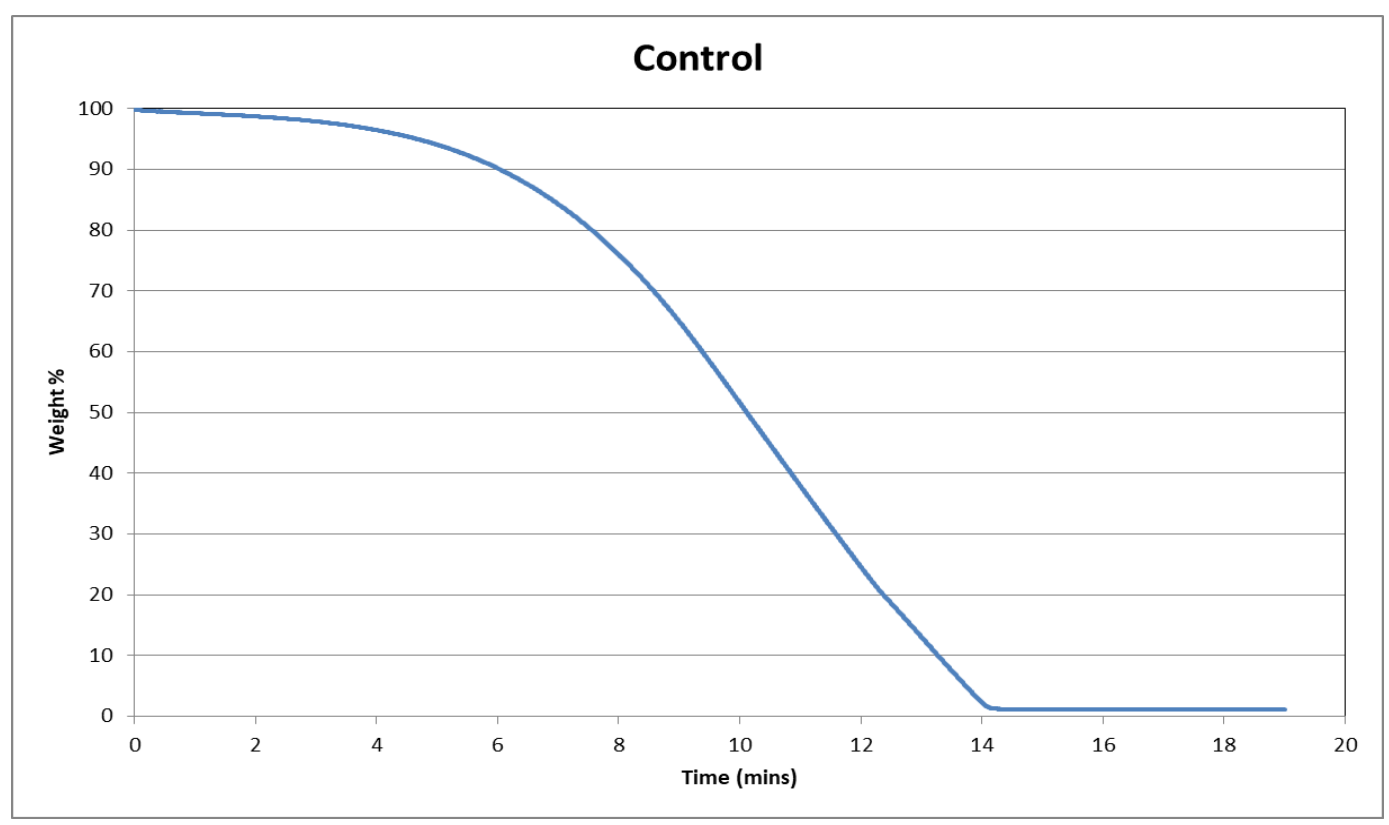

Figure 4.3.1. Shows the evolution of mass loss in response to time in minutes at 100 ${ }^{\circ} \mathrm{C}$. After 15 minutes $98 \%$ of the water has evaporated from the PEDOT:PSS sample 


\subsection{Results and discussio}

The highest electrical conductivity recorded in this chapter corresponds to the PEDOT:PSS film treated with 1-methyl-2-pyridone (1100 S cm-1). In some studies, it may be referred to as N-methyl pyrrolidone (NMP). ${ }^{329330}$ A study reported an electrical conductivity for 1-methyl-2-pyridone as $50 \mathrm{~S} \mathrm{~cm}^{-1}$ at room temperature. ${ }^{331} \mathrm{In}$ the same study when fabricating the film at $120^{\circ} \mathrm{C}$ its conductivity was reported as $325 \mathrm{~S} \mathrm{~cm}^{-1}$. In a study by Matsumoto et al a solvent vapour treatment utilizing 1-methyl-2-pyridone was conducted on PEDOT:PSS films in which the film achieved an electrical conductivity of $236 \mathrm{~S} \mathrm{~cm}^{-1}{ }^{329}$ In another study whereby $20 \% \mathrm{v} / \mathrm{v}$ 1-methyl-2-pyridone to PEDOT:PSS solution was deposited on glass substrate, the electrical conductivity for the film reached as high as $486.1 \mathrm{~S} \mathrm{~cm}^{-1} .{ }^{332} \mathrm{~A}$ study by Nasiri et al, looked into the effects of treating PEDOT:PSS with NMP at different lengths of time. An optimal electrical conductivity of around $325 \mathrm{~S} \mathrm{~cm}^{-1}$ for the film treated for less than 1 minute was realized. ${ }^{333}$

Figure 4.5.1 shows the thermoelectric properties of pristine PEDOT:PSS (a) and 1-methyl-2-pyridone (b) films. Figure 4.5.1 shows (a), the electrical conductivity of pristine PEDOT:PSS is as low $\left(<4 \mathrm{~S} \mathrm{~cm}^{-1}\right)$ at $60{ }^{\circ} \mathrm{C}$ and as high as $5.4 \mathrm{~S} \mathrm{~cm}^{-1}$ at 215 ${ }^{\circ} \mathrm{C}$. Compared to the electrical conductivity of the 1-methyl-2-pyridone film (Figure 4.5.1 (b) peaking as $1100 \mathrm{~S} \mathrm{~cm}^{-1}$ at $40{ }^{\circ} \mathrm{C}$, it is evident that the electrical conductivity of PEDOT:PSS can be significantly improved by treatment with the solvent. The preparation of the film can be found in (chapter 3.2.1). If a comparison is made between the PF of the two films, the PF in (Figure 4.5.1 (b) is superior throughout the measured temperature range than the control film (Figure 4.5.1 (a). The PF for the 1methyl-2-pyridone treated film at $40{ }^{\circ} \mathrm{C}$ is $24 \mu \mathrm{W} \mathrm{m}{ }^{-1} \mathrm{~K}^{-2}$ which is significantly higher than that of pristine PEDOT:PSS with a power factor of $0.07 \mu \mathrm{W} \mathrm{m} \mathrm{m}^{-1} \mathrm{~K}^{-2}$ at the same temperature. The power factor peaks at $200{ }^{\circ} \mathrm{C}$ for 1-methyl-2-pyridone film as $33 \mu \mathrm{W}$ $\mathrm{m}^{-1} \mathrm{~K}^{-2}$ meanwhile for the pristine PEDOT:PSS it peaks at only $0.43 \mu \mathrm{W} \mathrm{m} \mathrm{m}^{-1} \mathrm{~K}^{-2}$. This large difference in PF is mostly due to the difference in electrical conductivity between the two films. Because the power factor is the product of the electrical conductivity and Seebeck coefficient squared ( $\mathrm{PF}=S^{2} \sigma$ ) derived from chapter 1.2, Equation (1), as observed in Figure 4.5.1 (a) and (b) the Seebeck coefficient for both films is within a similar range of each other. The Seebeck coefficient at $40{ }^{\circ} \mathrm{C}$ is $13 \mu \mathrm{V} \mathrm{K}^{-1}$ and 15 
$\mu \mathrm{V} \mathrm{K} \mathrm{K}^{-1}$ for the pristine PEDOT:PSS and the 1-methyl-2-pyridone treated film, respectively. The Seebeck coefficient increases almost linearly for pristine PEDOT:PSS from $40{ }^{\circ} \mathrm{C}$ to $180{ }^{\circ} \mathrm{C}$ and then follows an exponential increment from $180{ }^{\circ} \mathrm{C}$ to $220^{\circ} \mathrm{C}$. For the 1-methyl-2-pyridone however, as shown in Figure 4.5.1(b), the Seebeck coefficient increases from $15 \mu \mathrm{V} \mathrm{K}^{-1}$ at $40{ }^{\circ} \mathrm{C}$ and peaks at $16 \mu \mathrm{V} \mathrm{K}^{-1}$ at $120{ }^{\circ} \mathrm{C}$ before decreasing to $14 \mu \mathrm{V} \mathrm{K}^{-1}$ at $165{ }^{\circ} \mathrm{C}$ then increasing exponentially thereafter. The boiling point of NMP is $202{ }^{\circ} \mathrm{C}$, from Figure $4.5 .1 \mathrm{~b}$ ) above $150{ }^{\circ} \mathrm{C}$, there is a dip in Seebeck coefficient which then begins to exponentially increase by remain relatively low above $180^{\circ} \mathrm{C}$. This could be attributed to the fact that the film was annealed at $120^{\circ} \mathrm{C}$ whereby some solvent was not evaporated. This evaporation continues until $180^{\circ} \mathrm{C}$ whereby the sample is still changing. A possible reason for the lack of increase in S indicates that NMP does not affect the oxidation state of PEDOT:PSS which has been linked to improved S. This slight dip in the Seebeck coefficient is attributed to the dip in the PF for 1-methyl-2-pyridone film which is not present for the pristine PEDOT:PSS film. The variation in the electrical conductivity for the 1-methyl-2-pyridone could be attributed to temperature a dependent charge carrier transport. 334,335

Figure 4.5.2 depicts the electrical conductivity of the 4 films derived from the $5 \% \mathrm{v} / \mathrm{v}$ solvent to PEDOT:PSS solution treatment. The electrical conductivity of the dimethyl sulfoxide treated film is $800 \mathrm{~S} \mathrm{~cm}^{-1}$ at $40{ }^{\circ} \mathrm{C}$. This value is consistent and within the range reported in literature. ${ }^{212,299,316,336}$ The electrical conductivity for ethylene glycol is $550 \mathrm{~S} \mathrm{~cm}^{-1}$ at $40{ }^{\circ} \mathrm{C}$ which is also within the range of values reported in literature. ${ }^{337}$ The electrical conductivity value for pristine PEDOT:PSS Figure 4.5.1 (a) used as a control in this study is in line with values observed in literature around $3.8 \mathrm{~S} \mathrm{~cm}^{-1}$ at $40{ }^{\circ} \mathrm{C}^{338}$ As observed in Figure 4.5.2 there is an inverse relationship between the electrical conductivity and the corresponding measurement temperature for three of the films, (ethylene glycol, dimethyl sulfoxide, and 1-methyl-2-pyridone). The inverse relationship suggests that these films do not primarily exhibit a thermally activated/assisted hopping mechanism regarding the charge carrier transport in the temperature range measured. In this thesis, some films will be referred to as (nonthermally assisted/activated hopping) to signify films that have a negative dependence on temperature regarding their electrical conductivity meanwhile films that have a 
positive dependence on temperature will be dubbed (thermally assisted/activated hopping).

a)
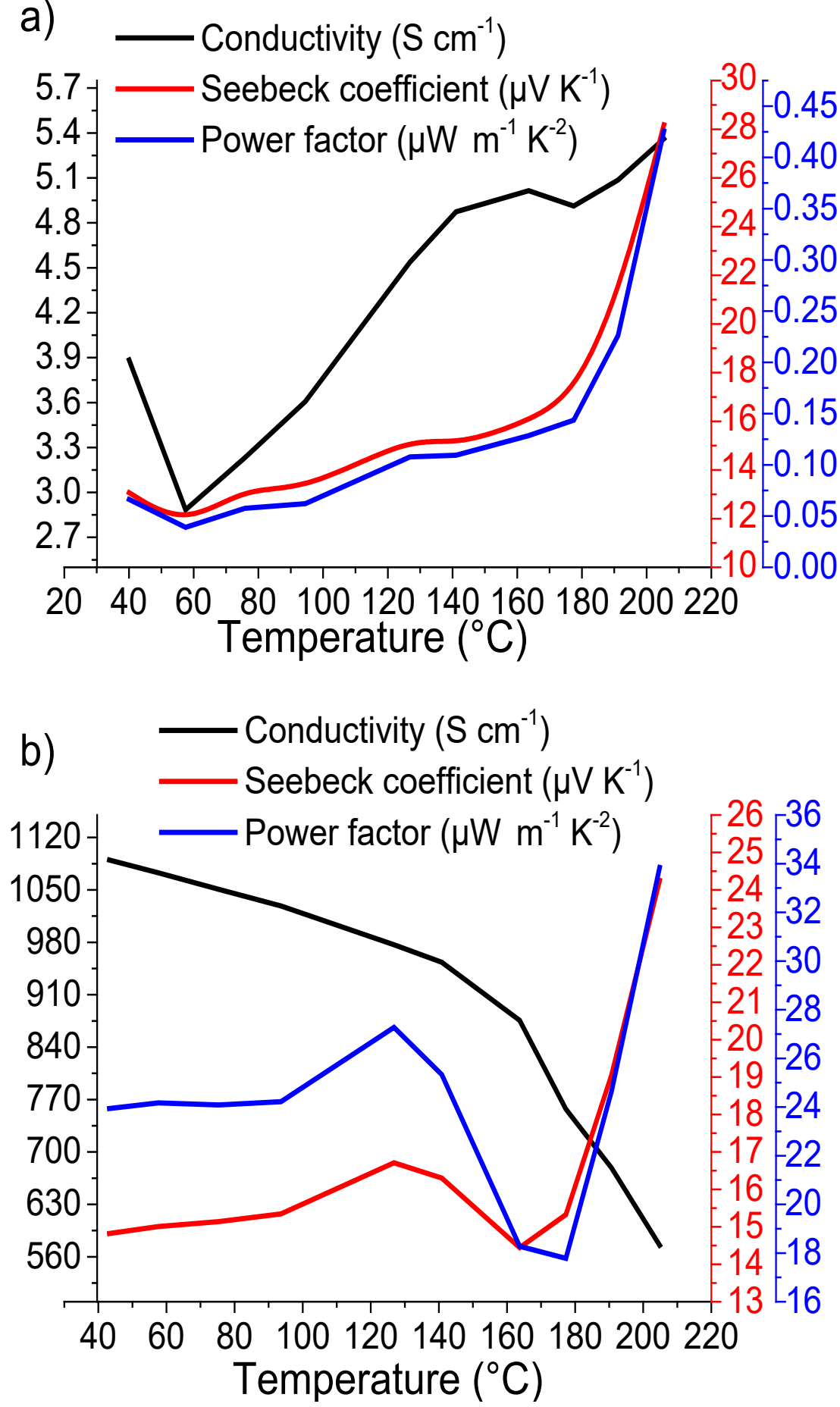
Figure 4.5.1, Seebeck coefficient, electrical conductivity, and Power factor of pristine PEDOT:PSS (a) film and 1-methyl-2-pyridone (b) treated films across a temperature range $40-200^{\circ} \mathrm{C}$.

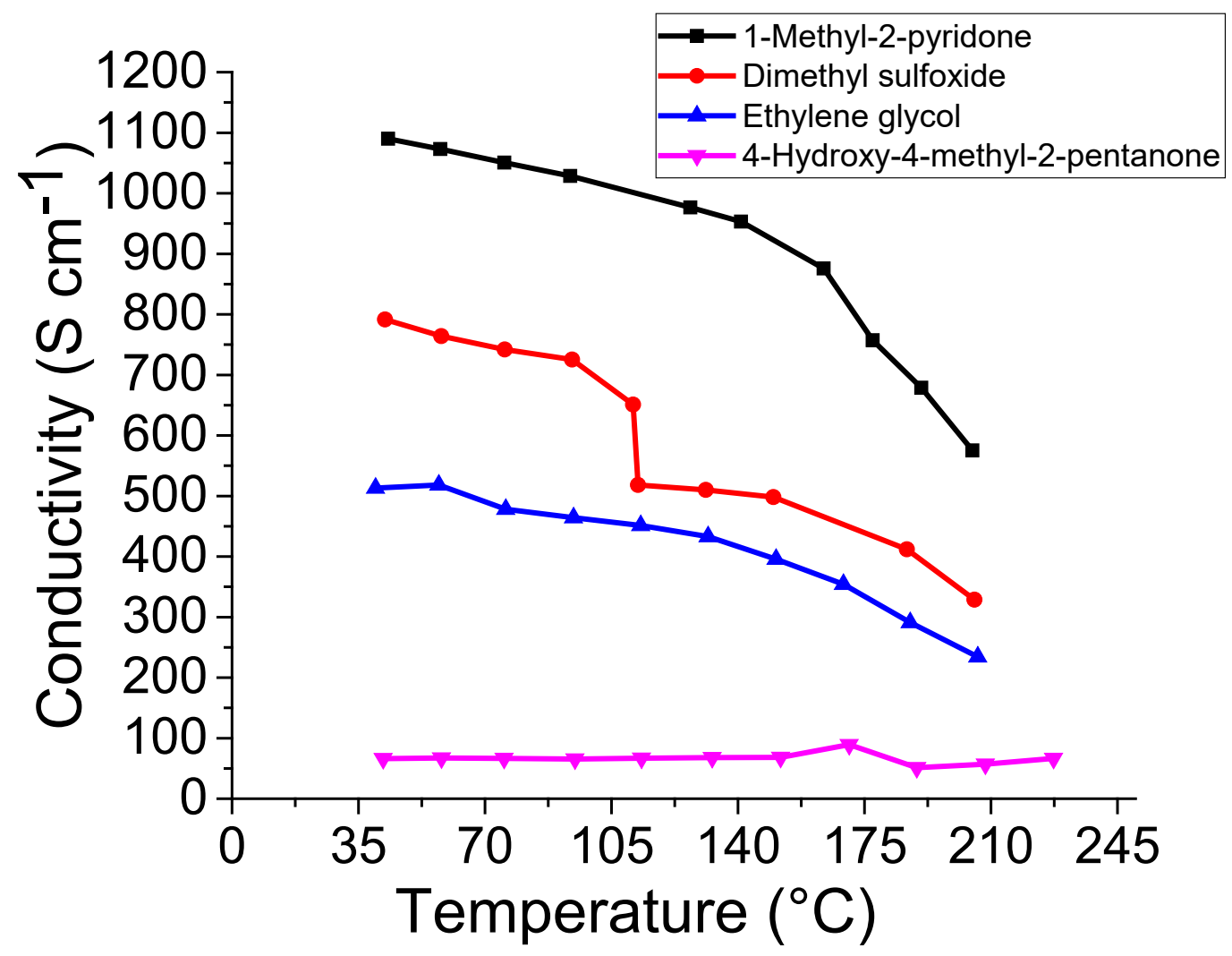

Figure 4.5.2. The electrical conductivity of 4 solvent treated (non-thermally assisted) PEDOT:PSS films across the temperature range $\left(40{ }^{\circ} \mathrm{C}\right.$ to $\left.250{ }^{\circ} \mathrm{C}\right)$ that showed a neutral or negative correlation between electrical conductivity and temperature.

This is a simplified expression used to analyse temperature dependence but it's routed in Motts formalisms for variable range hopping mechanism (VHR). ${ }^{110,220,335,339}$ Considering this, for the remainder this chapter these films will be represented as (nonthermally assisted hopping). In literature such conductivity has been ascribed as metallic conductivity. This is because across $40{ }^{\circ} \mathrm{C}$ to $250{ }^{\circ} \mathrm{C}$ temperature range there is no pattern observed whereby increased temperature yields a higher conductivity. A high $6200 \mathrm{~S} \mathrm{~cm}^{-1}$ PEDOT film was achieved due to solvent shearing exhibiting a metallic conductivity as evidenced by the decrease in conductivity as the temperature increases. ${ }^{341}$ Although it is arguable that the films could be yielding a lower electrical conductivity due to thermal instability, this is an unlikely explanation because studies show that PEDOT:PSS is stable up to $185^{\circ} \mathrm{C} .{ }^{340,342}$ In a study conducted by Caillou et 
al to obtain a relationship between conductivity and temperature, it was discovered the PEDOT:PSS film had an irreversible loss of conductivity above $200{ }^{\circ} \mathrm{C} .{ }^{342}$ Therefore, even if these PEDOT:PSS derivatives may be differently stable to the pristine films presented in the study, it is unlikely that the solvent treated films begin to deform at room temperature upon measurement. It is known that PEDOT:PSS sometimes exhibits metallic type temperature dependent (positive) conductivity or in some cases depending on the doping state exhibit an electrical conductivity that is less affected by temperature at low temperatures (room temperature). ${ }^{342}$ Figure 4.5.3 however shows that the propylene glycol, propylene carbonate, pristine PEDOT:PSS, 1-4 dioxane, and the 1-methyl-2-propanone exhibit a positive linear relationship between the electrical conductivity and the corresponding measured temperature.

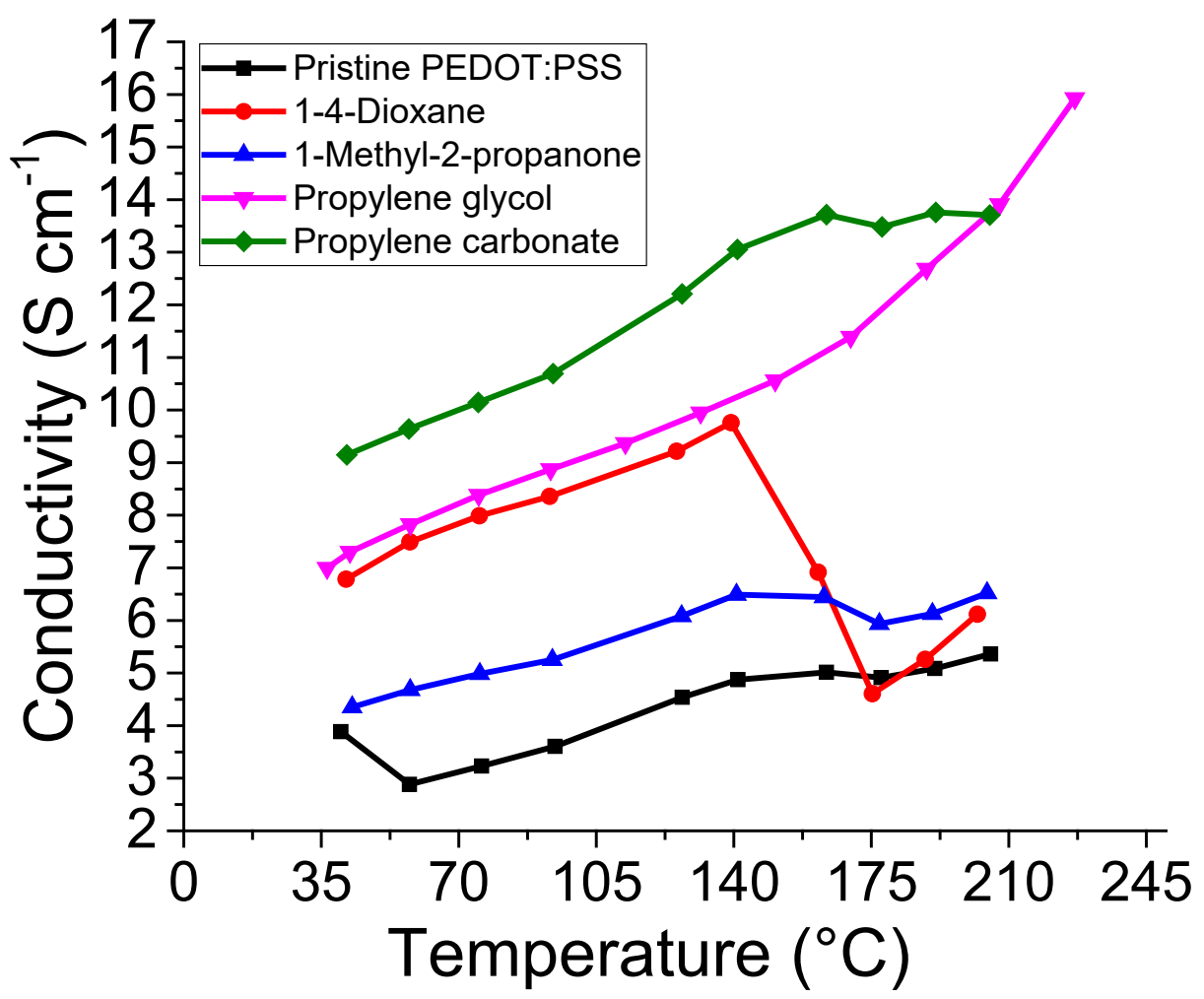

Figure 4.5.3 The electrical conductivity for five solvent treated (thermally assisted hopping) PEDOT:PSS films that showed a positive correlation between electrical conductivity and temperature across the temperature range $\left(40^{\circ} \mathrm{C}\right.$ to $\left.250^{\circ} \mathrm{C}\right)$. 
In this situation the trend suggests a thermally assisted hopping mechanism is a likely partial explanation for the charge carrier transport for these materials. At higher temperatures, the carrier transport is improved as more charge carriers around the Fermi level obtain enough energy to hop between higher energy levels. This leads to a statistically higher chance of the carriers to hop onto the conductive band giving rise to higher conductivity. ${ }^{99}$ The 1-4-dioxane however shows an increase in electrical conductivity up to $140{ }^{\circ} \mathrm{C}$ before decreasing. This dip appears to indicate thermal instability at higher temperatures. ${ }^{342}$ This is unlikely to be due to the 1-4 dioxane degrading into other compounds because its degradation has been shown to occur at temperatures above $350{ }^{\circ} \mathrm{C} .{ }^{345} \mathrm{It}$ is possible then that during film measurement that the solvent is acting on the film however as the temperature increases past $140{ }^{\circ} \mathrm{C}$ the solvent mostly evaporates. It appears that all treated films have either slightly or significantly higher electrical conductivity than the pristine PEDOT:PSS thus giving evidence that solvent treatment is a powerful tool to be utilized in increasing electrical conductivity of PEDOT:PSS. From these 4 films, the propylene carbonate shows the highest electrical conductivity of $8 \mathrm{~S} \mathrm{~cm}^{-1}$ at $40{ }^{\circ} \mathrm{C}$ and increasing up to $13 \mathrm{~S} \mathrm{~cm}^{-1}$ at $210{ }^{\circ} \mathrm{C}$. This is followed by propylene glycol which starts at $6 \mathrm{~S} \mathrm{~cm}^{-1}$ and reaches 13 $\mathrm{S} \mathrm{cm}^{-1}$ at $210{ }^{\circ} \mathrm{C}$ while peaking with $17 \mathrm{~S} \mathrm{~cm}^{-1}$ at $235{ }^{\circ} \mathrm{C}$. The 1-4-dioxane sample shows a similar trend and nearly matches the conductivity values for the propylene glycol except for the observed dip. For the 1-methyl-2-propanone the electrical conductivity is like that of PEDOT:PSS although slightly higher at $5 \mathrm{~S} \mathrm{~cm}^{-1}$ at $40{ }^{\circ} \mathrm{C}$ which increases slightly to $6 \mathrm{~S} \mathrm{~cm}^{-1}$ at $210^{\circ} \mathrm{C}$. As shown in Figure 4.5.2 the film treated with 4-hydroxy-4-methyl-2-propanone shows a stable non-significant change of electrical conductivity. In this film it is difficult to know if it is thermally assisted charge carrier transport as heat appears to have a net neutral effect on the conductivity. The conductivity for this film however is higher than that of the other 4 films shown in Figure 4.5.3 and but lower than all films in Figure 4.5.2. The conductivity does

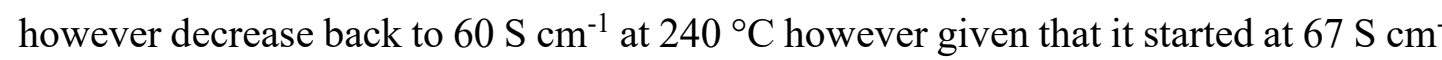
${ }^{1}$ this dip is not significant when compared to the dips of 1-methyl-2-pyridone in which although the electrical conductivity is high, it drops by half at higher temperatures above $200{ }^{\circ} \mathrm{C}$.

Figure 4.5.4 depicts the Seebeck coefficient for the 3 (non-thermally assisted hopping) and 1 neutral film. The positive sign on the Seebeck coefficient is indicative 
of p-type semiconductor characteristics. A general upward trend whereby the Seebeck coefficient is positively correlated with temperature is observed for all films. As shown in Figure 4.5.1 (a) the pristine PEDOT:PSS has slightly fewer fluctuations in Seebeck coefficient and therefore a smoother trend is observed. Although there are some fluctuations in which some films such as ethylene glycol and 4-hydroxy-4-methyl-2propanone.

Furthermore, these apparent fluctuations except for the dimethyl sulfoxide whereby at $118^{\circ} \mathrm{C}$ shows a significant increase in the Seebeck coefficient up to $24 \mu \mathrm{V}$ $\mathrm{K}^{-1}$ are within an acceptable range of error. It is important to note that these apparent fluctuations appear as such due to the small values measured here being nearer to the error amount of the measurement. The small changes in the Seebeck coefficient are largely similar for all films with some exceptions. It was later discovered after many repeats that the ZEM-3 system had difficulty measuring the Seebeck coefficient for many doped samples around $120^{\circ} \mathrm{C}+$ or $-8^{\circ} \mathrm{C}$. An exact answer to elucidate the reason was not realized in this project however considering this, the Seebeck coefficient of PEDOT:PSS doped samples in subsequent chapters was measured at 130 ${ }^{\circ} \mathrm{C}$ instead of $120{ }^{\circ} \mathrm{C}$. Figure 4.5.4 depicts the Seebeck coefficient of the (nonthermally assisted hopping) films across a temperature range of $40{ }^{\circ} \mathrm{C}$ to $240{ }^{\circ} \mathrm{C}$. Figure 4.5.5 shows the Seebeck coefficient against temperature for the thermally assisted hopping films. 


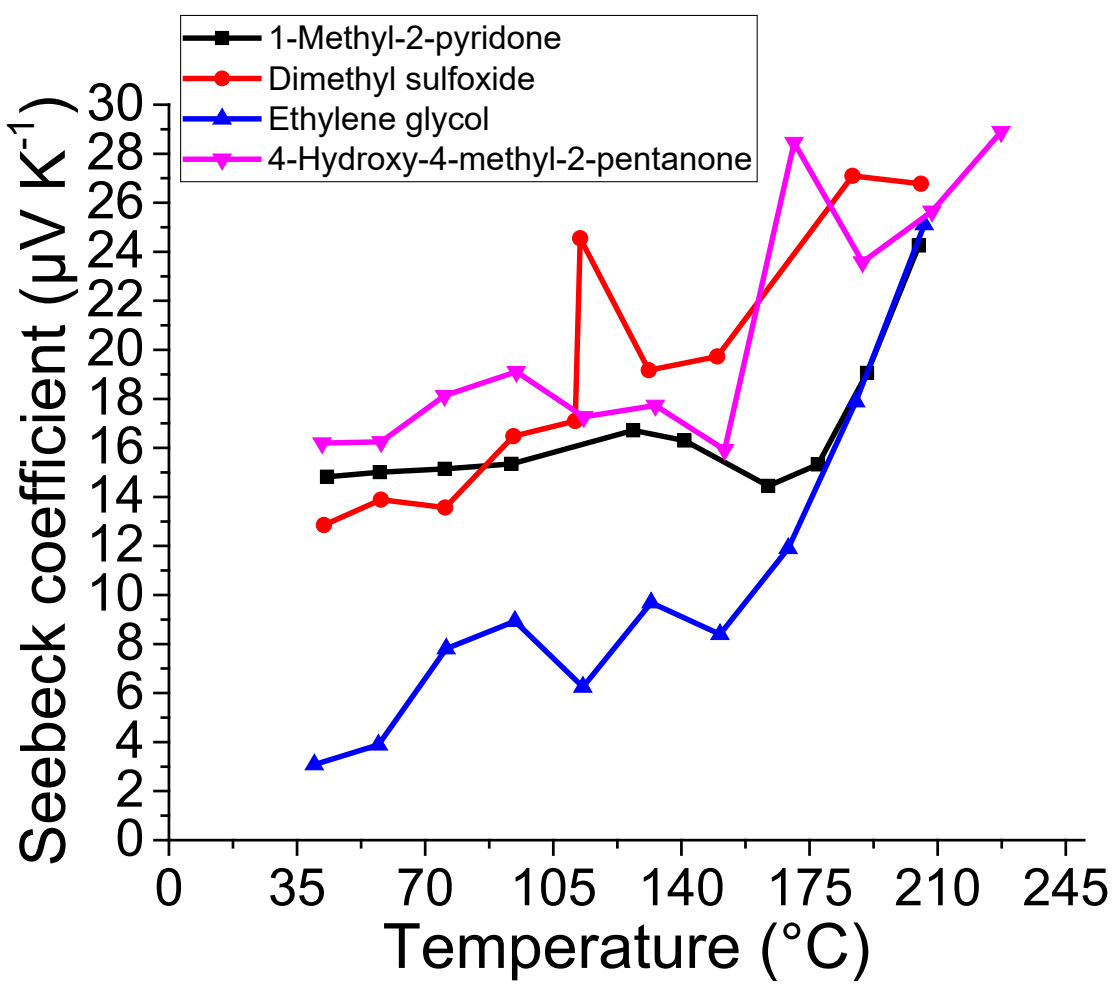

Figure 4.5.4 Shows the Seebeck coefficient at the measured temperature for the films that showed a net positive electrical conductivity to temperature relationship observed in Figure 4.5.3 (thermally assisted hopping).

Figure 4.5.5 shows that there is a general trend in the Seebeck coefficient improving at higher measurement temperatures. Except for propylene carbonate, the remaining films show a Seebeck coefficient within a similar range of each other. A possible reason why propylene carbonate's Seebeck coefficient not rising as much could be linked to its electrical conductivity increasing more than the other films. Comparing the Seebeck coefficient derived from the films in Figure 4.5.4 and 4.5.5 most films appear to not have a Seebeck coefficient significantly affected by solvent treatment relative to the control (pristine PEDOT:PSS). Therefore, solvent doping appears to selectively improve the electrical conductivity with minimal positive effects on the Seebeck coefficient and in some cases minor negative effects on the Seebeck coefficient. Still, as shown it appears solvent treatment may change carrier transport mechanism of PEDOT:PSS. A hypothesis to explain the effect on carrier mechanism by different solvents could be due to the whether the solvent primarily or significantly affects the physical orientation of PEDOT chains through improved carrier mobility which does not affect the Seebeck coefficient directly or if the transport mechanism is 
more significantly affected by electron doping/changing the molecular structure in such a way that the carrier concentration is affected. If the carrier concentration is increased slightly then it may explain the slight decrease in the Seebeck coefficient. Further studies would be required to see if the change or carrier transport mechanism can occur after solvent doping in conjunction with another treatment that improves the Seebeck coefficient. This may prove useful in designing strategies to decouple the electrical conductivity from the Seebeck coefficient.

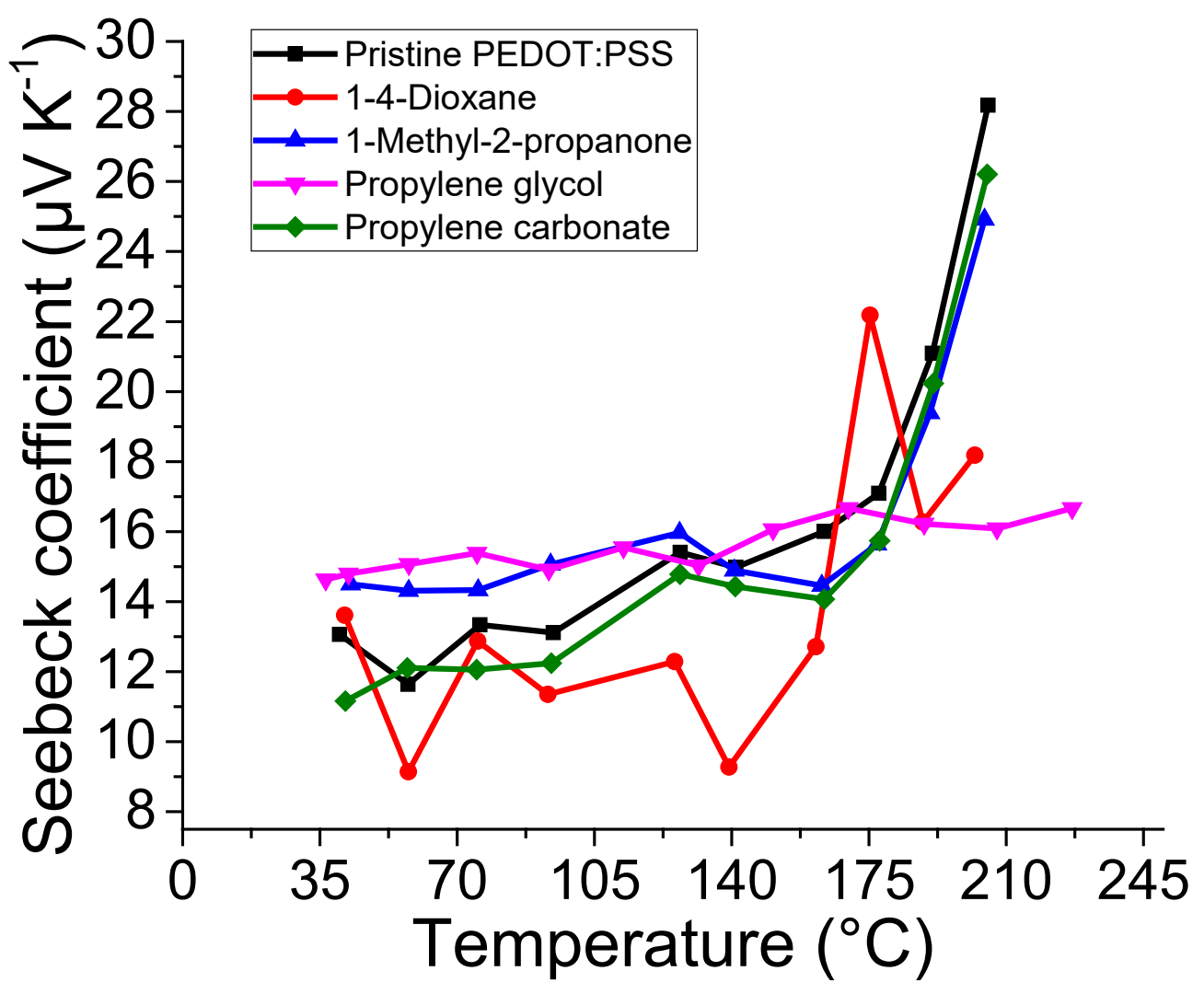

Figure 4.5.5. Seebeck coefficient of the 5 (thermally assisted hopping) films treated with various solvents across a temperature range of $40{ }^{\circ} \mathrm{C}$ to $>200{ }^{\circ} \mathrm{C}$

Figure 4.5.6 (a) and (b) depicts the power factor for all the nine films used in this study. Despite the high electrical conductivity observed for ethylene glycol treated film (Figure 4.5.4), the power factor at the lower temperatures for the film is low. This is due to the low Seebeck coefficient of $3 \mu \mathrm{V} \mathrm{K}^{-1}$ at $40^{\circ} \mathrm{C}$ as shown in figure 4.5.6 (a). Consequently, the power factor for ethylene glycol film is extremely low at $40^{\circ} \mathrm{C}$ with 
a value of $0.5 \mu \mathrm{W} \mathrm{m} \mathrm{m}^{-1} \mathrm{~K}^{-2}$ and only rises above $1 \mu \mathrm{W} \mathrm{m} \mathrm{m}^{-1} \mathrm{~K}^{-2}$ at higher temperatures such as $80{ }^{\circ} \mathrm{C}$ where its $\mathrm{PF}$ is $4 \mu \mathrm{W} \mathrm{m} \mathrm{m}^{-1} \mathrm{~K}^{-2}$. The power factor for ethylene glycol continues to increase and peaks at $215^{\circ} \mathrm{C}$ with a value of $17 \mu \mathrm{W} \mathrm{m} \mathrm{K}^{-1}$.
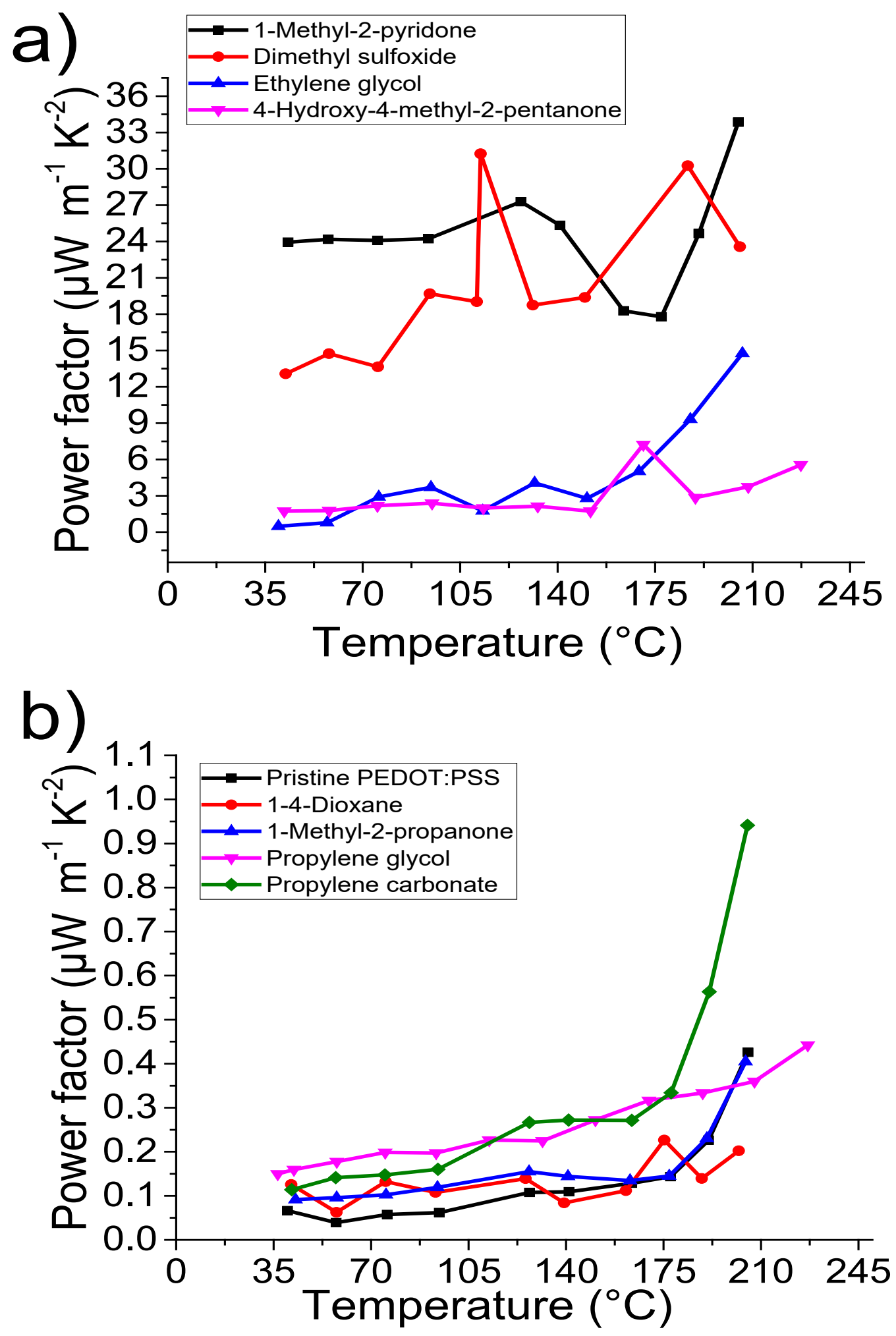
Figure 4.5.6 (a) The Power factor at a given temperature for the (non-thermally assisted hopping films and neutral film), meanwhile (b) corresponds to the power factor for the 5 films with a positively correlated electrical conductivity and measurement temperature (thermally assisted hopping) from $40^{\circ} \mathrm{C}$ to $235^{\circ} \mathrm{C}$.

Because the peak PF is at such a high temperature $\left(215^{\circ} \mathrm{C}\right)$ the viability of the ethylene glycol film as a functional thermoelectric material would be limited since it's been reported that after several repeated thermoelectric runs (cycles) above $200{ }^{\circ} \mathrm{C}$ may lead to some polythiophene chains in PEDOT to undergo a Diels-Alder reaction ( $\mathrm{SO}_{2}$-extrusion). ${ }^{18,342-344}$ It's possible that the $\mathrm{S}$ in some films may increase when undergoing the $\mathrm{SO}_{2}$-extrusion because as the PEDOT units degrade an overall lowering of the carrier concentration would also occur in the bulk film which as expressed in chapter 1.2 equation 4, a lower carrier concentration is related to higher S. This thermal degradation at high temperature invariably limits the operation temperature of PEDOT based films to under $200{ }^{\circ} \mathrm{C}$ and if considering longevity of the film in functional devices perhaps temperatures lower than $185^{\circ} \mathrm{C}$ would be more ideal baseline. Therefore, the ethylene glycol treated film remains less viable as a thermoelectric material than the dimethyl sulfoxide, or 1-methyl-2-pyridone which as evidenced in Figure 4.5.6 (a) whereby they exhibit significantly higher power factors across the measured temperature range.

As shown in Figure 4.5.4 and 4.5.5 the remainder of the other films exhibit a Seebeck coefficient measurement within a similar range of each other. Figure 4.5.5 shows that at $40{ }^{\circ} \mathrm{C}$ the pristine PEDOT:PSS, 1-4-dioxane and dimethyl sulfoxide treated films, all exhibit a Seebeck coefficient of $12 \mu \mathrm{V} \mathrm{K} \mathrm{K}^{-1}$. The Seebeck coefficient of the dimethyl sulfoxide film incrementally rises then slightly larger values can be observed throughout the temperature range whereby it peaks as $27 \mu \mathrm{V} \mathrm{K}^{-1}$ at $215^{\circ} \mathrm{C}$. The Seebeck coefficient of the pristine PEDOT:PSS however peaks slightly higher at the same temperature with a value of $28 \mu \mathrm{V} \mathrm{K}^{-1}$ and 1-4-dioxane peaks at $180^{\circ} \mathrm{C}$ with a value of $23 \mu \mathrm{V} \mathrm{K}^{-1}$. Still, the power factor for the dimethyl sulfoxide film due to its much higher electrical conductivity yields a significantly higher power factor than the pristine PEDOT:PSS or 1-4-dioxane. Therefore, when looking at Figure 4.5.6 the power factor for the dimethyl sulfoxide treated film is shown as $12 \mu \mathrm{W} \mathrm{m}{ }^{-1} \mathrm{~K}^{-2}$ at 40 ${ }^{\circ} \mathrm{C}$, meanwhile the 1-4-dioxane and pristine PEDOT:PSS films are depicted as 0.13 
$\mu \mathrm{W} \mathrm{m} \mathrm{m}^{-1} \mathrm{~K}^{-2}$ and $0.05 \mu \mathrm{W} \mathrm{m} \mathrm{K}^{-1} \mathrm{~K}^{-2}$ respectively. The power factor for the dimethyl sulfoxide treated film peaks at $120^{\circ} \mathrm{C}$ with $33 \mu \mathrm{W} \mathrm{m}{ }^{-1} \mathrm{~K}^{-2}$ showing potential viability to be utilized in a functional thermoelectric device due to it being operable below the degradation temperature range for PEDOT:PSS. ${ }^{18}$ At $40{ }^{\circ} \mathrm{C}$ the propylene glycol film has a Seebeck coefficient of $15 \mu \mathrm{V} \mathrm{K}^{-1}$ which across the measured temperature profile doesn't rise beyond $16 \mu \mathrm{V} \mathrm{K}^{-1}$. Therefore, considering its electrical conductivity as it increases at higher temperatures the power factor starts low at $0.15 \mu \mathrm{W} \mathrm{m}^{-1} \mathrm{~K}^{-2}$ but rises to $0.4 \mu \mathrm{W} \mathrm{m} \mathrm{m}^{-1} \mathrm{~K}^{-2}$ at $230{ }^{\circ} \mathrm{C}$. For the 1-methyl-2-propanone, and propylene carbonate films the power factor is low at $40{ }^{\circ} \mathrm{C}$ observed as $0.13 \mu \mathrm{W} \mathrm{m}{ }^{-1} \mathrm{~K}^{-2}$ but still higher than that of the pristine PEDOT:PSS film showing an increased thermoelectric performance when utilizing dopants. The propylene carbonate film has a much higher PF above $1 \mu \mathrm{W} \mathrm{m}{ }^{-1} \mathrm{~K}^{-2}$ at $200{ }^{\circ} \mathrm{C}$, meanwhile the pristine PEDOT:PSS and 1-methyl2-propanone does not rise higher than $0.4 \mu \mathrm{W} \mathrm{m}^{-1} \mathrm{~K}^{-2}$ at comparable temperatures. The power factor is higher for the propylene carbonate due to it having higher electrical conductivity than the other two films as well as having no significant variation in the Seebeck between the other two films either.

\subsection{Morphology of PEDOT:PSS thin films}

Factors such as morphology, molecular orientation/ molecular chemistry as well stoichiometry between the PEDOT and PSS units in the polymer affect its electrical conductivity and Seebeck coefficient. Because all the solvents used this study improved the electrical conductivity of PEDOT:PSS based thin films albeit by different amounts, an optical microscopy study was conducted to ascertain some origins behind the improvement. The resolution for the micrographs allowed for features up to $1 \mu \mathrm{m}$ to be observed. Because PEDOT:PSS is a conductive nano ink, a scanning electron microscopy would have been able to in theory allow observations of finer details in the tens of nano meter scale, however, it was discovered during the study that features could not be differentiated in PEDOT:PSS based samples unless it was composited by nano particulates such as silver nano particles etc. Because the films in this study did not contain foreign solid particulates the optical micrograph was used instead. 
Figure 4.6.1 is a 2D representation of the structure of PEDOT:PSS. The PSS component is bonded to PEDOT through ionic and inter molecular interactions. In pristine PEDOT:PSS solutions most of the chains are in their coiled state. Figure 4.6.2 shows a diagram representing 3 different molecular orientations of PEDOT and PSS in the polymer. The core shelled conformation can be observed in Figure 4.6.2 (a), it is essentially a three-dimensional micelle like sphere of mixed, but mostly PEDOT core surrounded by a shell of PSS. ${ }^{346-348}$. This leads to a poorly conductive material when processed but due to the PSS's anionic nature allows for the polymer to be soluble in water. In the coiled structure the charge carriers are trapped within and between the polythiophene chains thereby leading to a statistically unfavourable charge carrier hopping to adjacent PEDOT. These transport inhibitions can be denoted as (traps). The insulative PSS region effectively acts as an inhibitor of charger carriers.

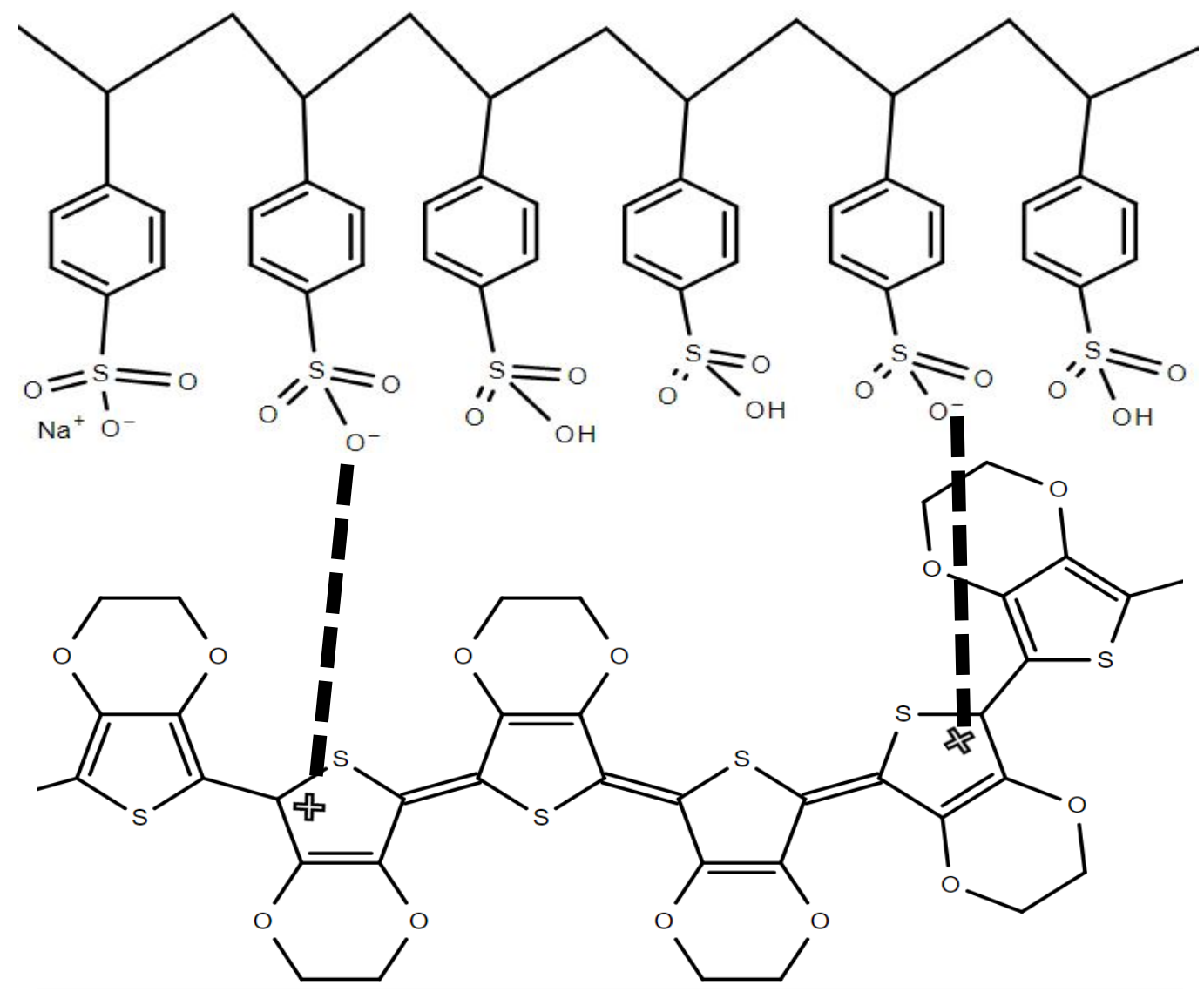


Figure 4.6.1 2D molecular structure of PEDOT and PSS and the subsequent ionic interactions. ${ }^{349}$

\section{PEDOT}

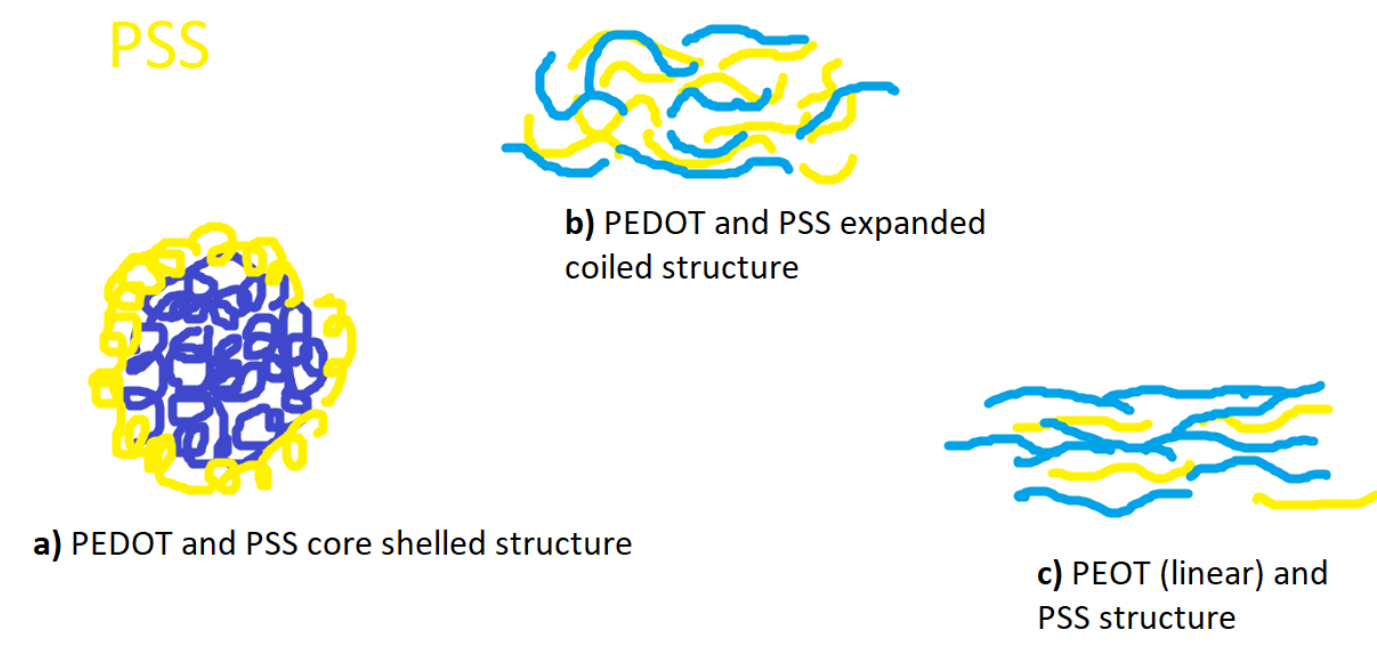

Figure 4.6.2 the different molecular orientations of PEDOT and PSS.

It is reasonable to postulate that the consequences (bundles) of core shelled structure (Figure 4.6.2 (a) ) could be observable as aggregated particulates which can be observed as pockets of defects when PEDOT:PSS is spun on a substrate. By utilizing an optical microscopy, it is possible to see if there is a relationship between number of microscopic defects and the electrical conductivity in the films. Figure 4.6.3 shows the different (molecular) resonance forms of the polythiophene chains in PEDOT. Figure 4.6.3 (a) is the neutral quinoid (b) neutral benzoid, (c) positive polaron, and (d) positive bipolaron. The PEDOT:PSS (Poly(3,4ethylenedioxythiophene)-poly(styrenesulfonate) solution as prepared in the PH1000 formulation used in this study is in the doped (fully oxidised) form (Figure 4.6.3 (a)/(b). In this state the majority of the polythiophene chains are comprised of bipolarons. Because all the PEDOT:PSS based films in this chapter exhibit a p-type characteristic whereby holes are the majority charge carrier as shown with the positive sign corresponding to the as measured Seebeck coefficient in Figure 4.5.6 (a) and (b) the polarons will be denoted with a prefix (positive) as stated in Figure 4.6 .3 (c) and (d). 


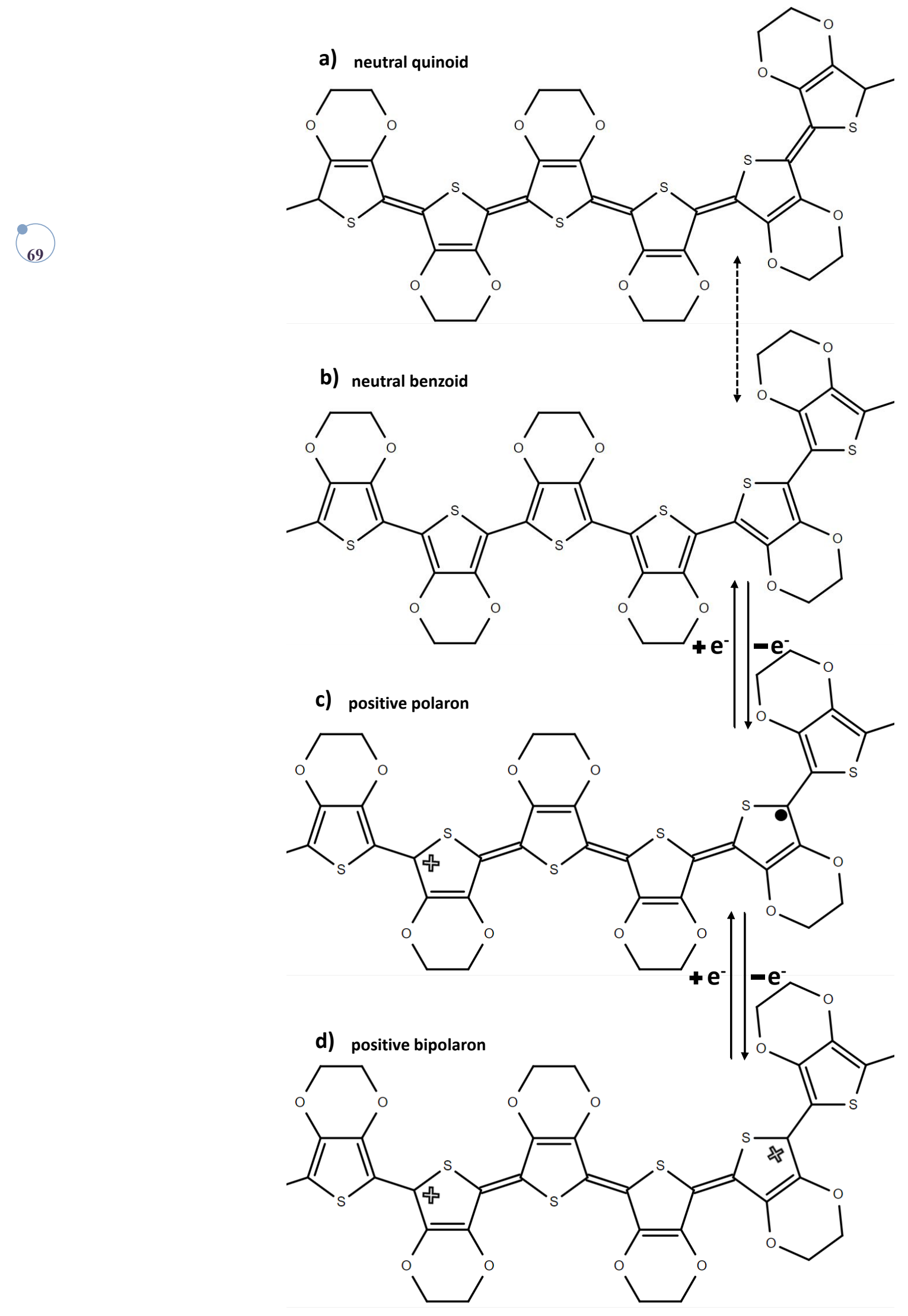


Figure 4.6.3 depicting the resonance forms of Poly(3,4-ethylenedioxythiophene) (PEDOT) thiophene back bone, (a) denotes the quinoid form, meanwhile (b) is the benzoid form. (c) and (d) represent the positive polaron and bipolaron oxidation states, respectively.

Figure 4.6.4 depicts the optical micrograph representing the (thermally assisted hopping) mechanism films.
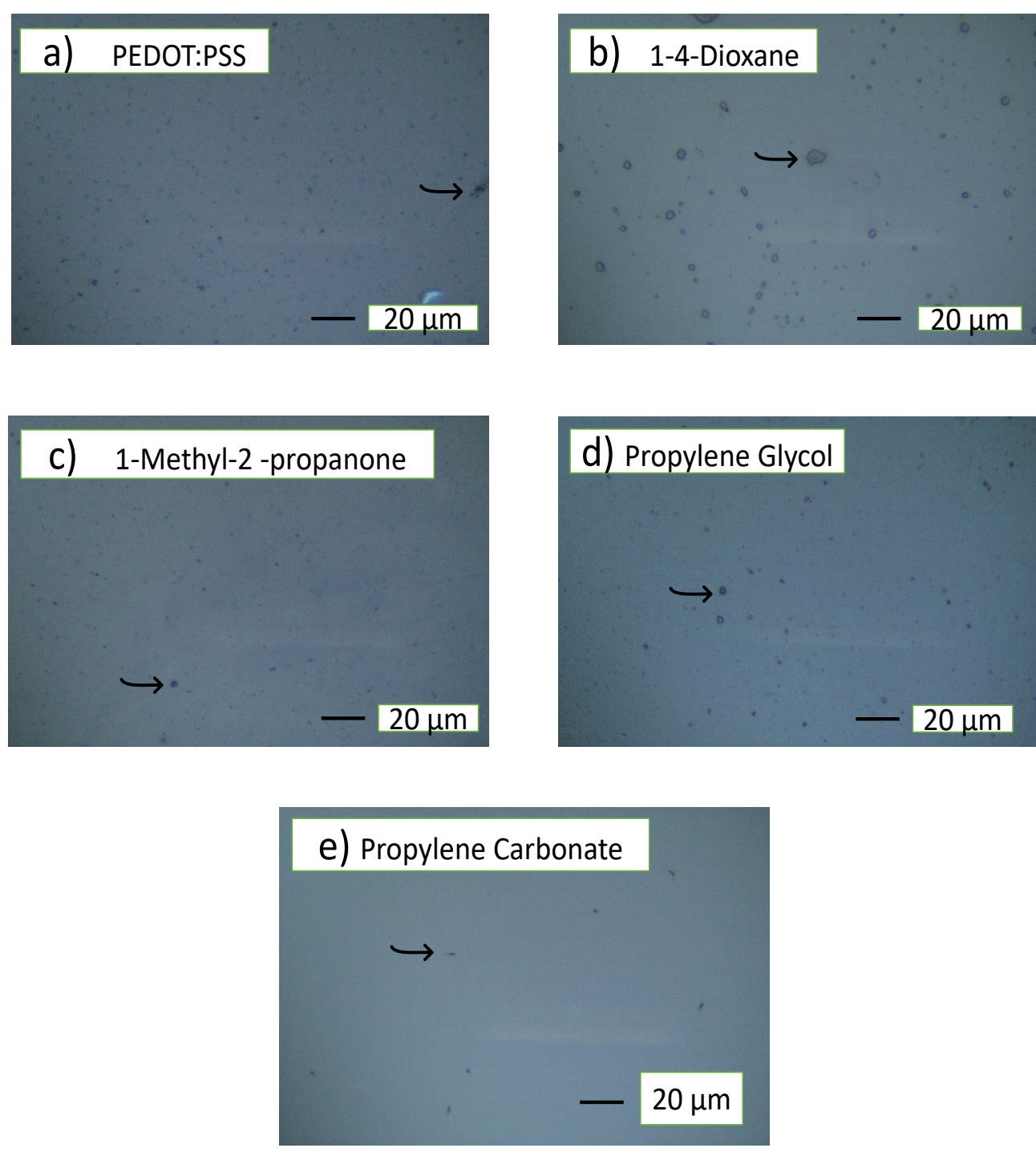

Figure 4.6.4 micrographs of the thermally assisted hopping mechanism films.

A light blue hue is observed for all films. This is typical for (doped) fully oxidised (bi-polaronic or partially oxidised mono-polaronic) films that are not 
composited with other solid particles (please refer to Figure 4.6.3 (c), and (d). It would be expected if the films were heavily reduced (dedoped) for a significantly deeper darker hue to be observed. ${ }^{350,351}$ Although the microscope colour may not represent the real colour of images, a primary image first analysed with a digital camera in a the same room other films were analysed at with the same lighting conditions and then compared for consistency with the images obtained from the microscope camera. The images appeared to be the same contrast. The hue observed in these films gives an inference that none of the films in Figure 4.6.4 are fully reduced (Figure 4.6.3 (a)/(b). Figure 4.6.5 depicts the optical microgram for the (thermally assisted hopping) mechanism films. The films have a blue hue in the same range as the non-thermally assisted films (figure 4.6.4). The colour implies that the films are not in their neutral (reduced) state and therefore inferring the polythiophene are in their fully oxidised or at least it in their partially oxidised state (please see Figure 4.6 .3 (c)/(d). This inference can be confirmed by oxidation state studies via UV-VIS and XPS. As shown in Figure 4.6.4/4.6.5 the black arrows represented in each film depicts structural defects. It is likely that the defects derive from slightly different reasons.
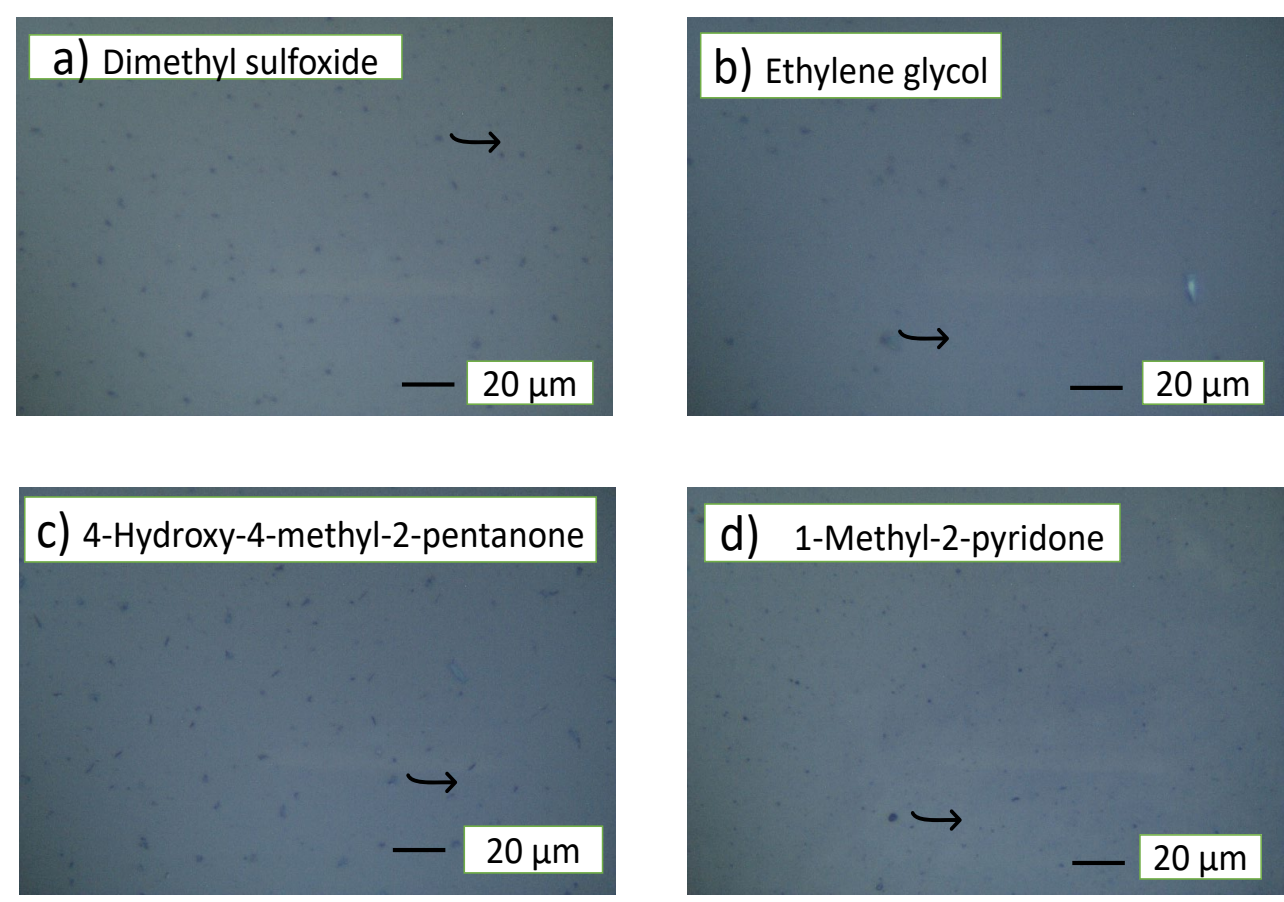

Figure 4.6.5.Micrograph with a 20 m scale bar for the 3 (thermally assisted hopping) and 1 neutral mechanism films. 
As shown in Figure 4.6.4 the original hypothesis was that the defects relating to pristine PEDOT:PSS derives from coiled PEDOT:PSS bundles. Following that logic, the (thicker) sections represent bundles of the film therefore their quantity may help elucidate some surface inhomogeneity. The bundles would then represent aggregated structures of PEDOT:PSS in the core shelled structure that is less conductive than the linear/ expanded coil structures. All films in Figure 4.6.4 except for 1-4-dioxane and to a lesser extent propylene glycol have a similar shape and appearance of defects to pristine PEDOT:PSS thereby indicating a similar defect origin as mentioned for the pristine PEDOT:PSS film. The two films, however, have round doughnut shaped defects exhibiting dark rings and discoloured cores. In these films the likely origin for those defects could derive from incomplete miscibility with the PEDOT:PSS solution. It was observed during mixing that there was some precipitation of clear a liquid on addition of both 1-4-dioxane and propylene glycol to the polymer in the respective vials. Although after shaking the vials briefly caused it to disappeared, it is possible during spin coating that the centrifugal force separated the polymer and the solvent at different rates leading to the ring effect which has been observed in literature. ${ }^{352-355}$ The films in Figure 4.6.5 show similar defect shape and general appearance to pristine PEDOT:PSS. If not for the poor electrical conductivity displayed by the propylene carbonate (Figure 4.6.4) it would be interesting to do further research since it displayed the most (pristine) defect free film owing to extremely low quantity of defects relative to any other film in either Figure 4.64 or 4.6.5 which includes PEDOT:PSS. There are differences in the number of defects at the scale of $<10 \mu \mathrm{m}$ as shown in Table 4.6.1.

Table 4.6.1 The approximated quantity of defects $>1 \mu \mathrm{m}$ in 9 PEDOT:PSS based films in the order of greatest to least as well as the corresponding electrical conductivity. The area is $160 \mu \mathrm{m}$ (length) by $90 \mu \mathrm{m}$ (width). The magnification is x 50.

\begin{tabular}{|l|l|l|l|}
\hline Film & Approximation of & Boiling point of & Electrical \\
& quantity of & solvent in each & conductivity \\
defects per 14400 & film & $\left(\mathrm{S} \mathrm{cm}^{-1}\right)$ at $40^{\circ} \mathrm{C}$ \\
$\mu \mathrm{m}^{2}$ & $\left({ }^{\circ} \mathrm{C}\right)$ & \\
& $(>10 \mu \mathrm{m})$ & & \\
\hline
\end{tabular}




\begin{tabular}{|l|l|l|l|}
\hline pristine PEDOT:PSS & 220 & 100 & 3.9 \\
\hline 1-methyl-2-pyridone & 192 & 250 & 1100 \\
\hline $\begin{array}{l}\text { 4-hydroxy-4-methyl- } \\
\text { 2-Pentanone }\end{array}$ & 120 & 166 & 66 \\
\hline 1-4-dioxane & 120 & 101 & 6.8 \\
\hline propylene glycol & 110 & 188 & 7 \\
\hline $\begin{array}{l}\text { 1-methyl-2- } \\
\text { propanone }\end{array}$ & 105 & 105 & 4.4 \\
\hline dimethyl sulfoxide & 100 & 189 & 792 \\
\hline ethylene glycol & 49 & 198 & 513 \\
\hline propylene carbonate & 9 & 240 & 9 \\
\hline
\end{tabular}

As shown in Table 4.6.1 and Figure 4.6.6 (a) there is no clear or emergent pattern to suggest that the quantity of defects at this scale have a significant effect on the electrical conductivity. The solvent that produced the film with the highest electrical conductivity (1-methyl-2-pyridone) also contained the highest number of defects. Several images were taken for each film and the images that represented the average quantity of defects were used for Table 4.6.1, as well as Figure 4.6.4 and 4.6.5. It is also important to note, the film with the lowest defects had a relatively low electrical conductivity. The lack of a correlation between quantity of defects and conductivity can be explained because the solvents used have different molecular structure thereby leading to different molecular changes in the PEDOT:PSS films that the optical micrograph cannot detect. In a very recent study by Karan et al, it was argued that within a conductive pathway in PEDOT:PSS the fastest pathway was the intra chain transport meanwhile the inter chain transport was the slowest. Although it was thought that defects may represent more of inter chain transport, due to PEDOT polymer chains being a few nano meters in length it is not possible for the optical images to detect the transport mechanism of PEDOT. If so, then it is not surprising that the number of defects was not correlated to the electrical conductivity. Because these films are treated by different solvents, chemical doping appears to have a more significant effect on the film's electrical conductivity than the defects observed in the optical microscopy images presented in the tables 4.6.1 and graphically presented in 
Figure 4.6.6 (a). If this is the case analysis using UV-VIS, Raman spectroscopy and XPS will corroborate the hypothesis.

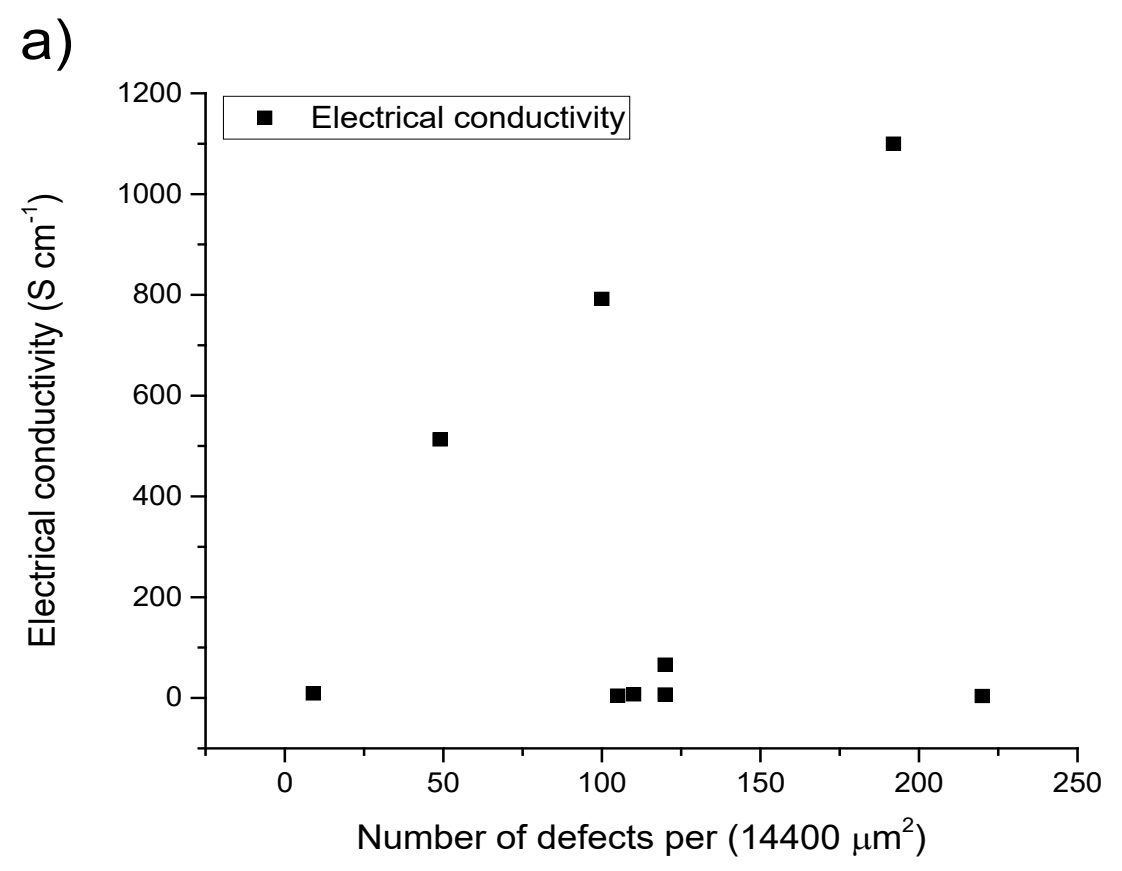

b)

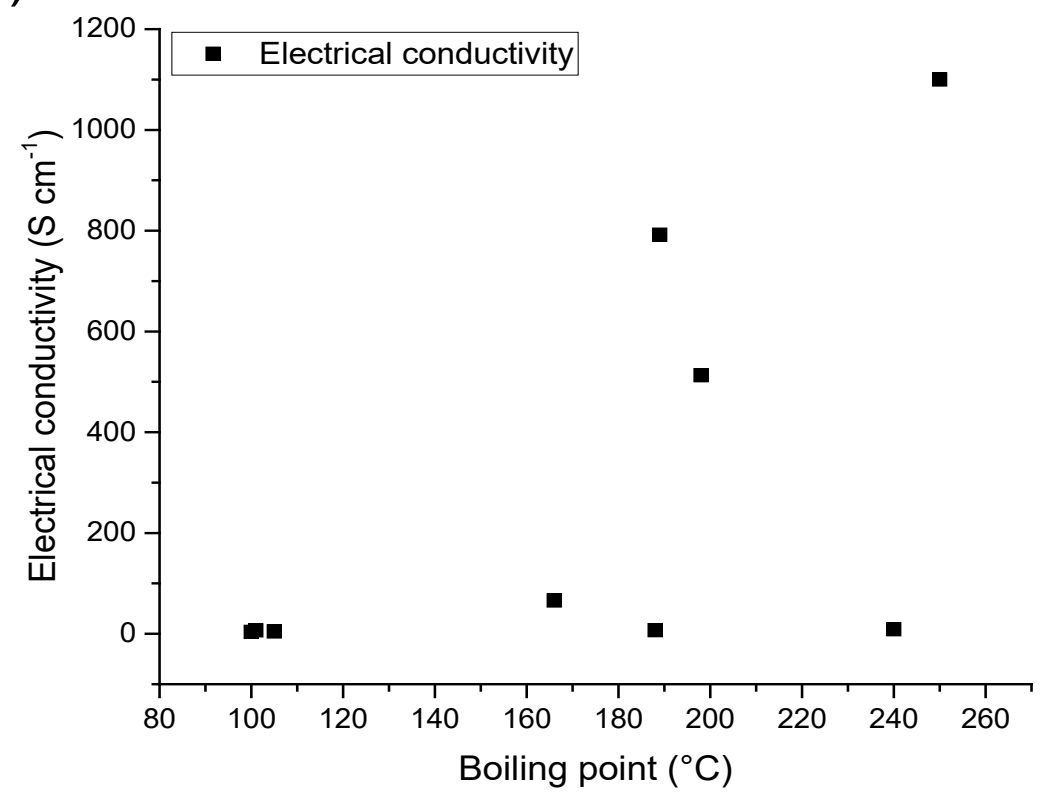

Figure 4.6.6 (a) depicts the electrical conductivity against the number of defects per $14400 \mu^{2}$, meanwhile (b) depicts the electrical conductivity against boiling point from the data in Table 4.6.1. 
Table 4.6.1 shows the boiling points of the various solvents used in this study. Figure 4.6.6 (b) shows the boiling point in relation to the electrical conductivity. It is difficult to argue for a clear relationship between the boiling point of the solvent and the electrical conductivity of the corresponding film. Even though the film with the highest boiling point also had the highest electrical conductivity (1-methyl-2-pyridone); the pattern is offset by propylene carbonate with the second highest boiling point $\left(240^{\circ} \mathrm{C}\right)$ but with only $9 \mathrm{~S} \mathrm{~cm}^{-2}$ and propylene glycol with a high boiling point $\left(188^{\circ} \mathrm{C}\right)$ but relatively low conductivity at $7 \mathrm{~S} \mathrm{~cm}^{-1}$.

\subsection{Molecular effects impacting electrical conductivity and Seebeck coefficient.}

Chemical treatments can induce changes in the oxidation state of polythiophene chains. (Please see Figure 4.6.3 for different oxidation states and molecular orientation of PEDOT). ${ }^{128,194,212,308}$ Subsequently these changes can be studied as mapped responses in the spectra obtained from (Raman, XPS, and UV-VisNIR instruments) to determine what, and to what extent the effects are linked to the improvements in both the Seebeck coefficient and electrical conductivity in the thin films. Figure 4.7.1 depicts the UV-Vis-NIR spectra for the 8 films treated with different solvents as well as the pristine PEDOT:PSS across $300 \mathrm{~nm}$ to $2400 \mathrm{~nm}$. The pristine PEDOT:PSS spectra (Figure 4.7.1 (a) is characteristic of PEDOT:PSS films as seen in literature. ${ }^{338,356}$ The extent of the absorption at each wavelength region $(600$, 900 , and above $1800 \mathrm{~nm}$ ) corresponds to the amount of polythiophene in either neutral, polaronic, or bipolaronic carrier state respectively (please see figure 4.6.3). ${ }^{139}$

As expressed in Figure 4.7.1 (a) there is a high absorption at $(1800 \mathrm{~nm})$ extending into the far NIR for the pristine PEDOT:PSS. This indicates that the majority of the polythiophene chains are predominated by bipolarons as charge carriers. The peak at the polaron region $(900 \mathrm{~nm})$ appears to be significantly smaller. This suggests that only a few polythiophene chains are in their polaron oxidation state. In the literature it is usually shown that in cases where the oxidation state changes to form more polarons a corresponding higher Seebeck coefficient is also observed when the 
original of the improvement is due to an oxidation state change. ${ }^{128,357}$ In the polaron peak for the pristine PEDOT the pattern does not deviate from most of the films as show in figure 4.7.1 (a)/(b). If the PEDOT:PSS was in a neutral (reduced) stated it would exhibit a high absorption at around $600 \mathrm{~nm}$ however as depicted in Figure 4.7.1 there is no distinct peak for pristine PEDOT:PSS said region thereby confirming the inferences made by analysing the colour grading of the optical images in chapter 4.6 (Figure 4.6.4/4.6.5).

There are some minor differences in the initial absorbance between the different films thereby indicating slight differences in film thickness as was measured by the Dektak surface profilometer. In this case the films exhibited a thickness between 50 and $60 \mathrm{~nm}$ with most films only having a $5 \mathrm{~nm}$ difference between each other. Since the UV-Vis-NIR spectra were not normalized it is not possible to make absolute inter film quantity comparisons. Still, it is possible to clearly observe when a film has a significant peak at the region of interest and make qualitative comparisons within and between each film. Figure 4.7.1 (a) represents the UV-Vis-NIR spectra for the (thermally assisted hopping) mechanism films. The pristine PEDOT:PSS, 1-4dioxane, 1-methyl-2-propanone exhibit similarly shaped spectrum. The propylene glycol and propylene carbonate however have spectra that deviate from the "usual" PEDOT:PSS spectrum appearance found in literature. ${ }^{171}$ Except for the propylene glycol and propylene carbonate films, a peak at the polaron region is observed for the other three films. The three films exhibit the same intensity at the polaron region as shown in (Figure 4.7.1 (a). The 1-4-dioxane, has the film with a medium bipolaron intensity in relation to the others. Depending on the mechanism of improved Seebeck coefficient, if there was a higher Seebeck coefficient observed in these films due to the solvent treatments, then there would either be a reduced bipolaron region and subsequent increase in the polaron and or neutral region. There are other ways to improve Seebeck coefficient without increasing the polaron region however these would involve ionic accumulation at the surface or nano incorporation of particles of rare earth minerals such as tellurium/selenium which exhibit naturally high Seebeck coefficient. $^{23,141,227,358}$ The Seebeck coefficients as confirmed by analysis of Figure 4.5.5 do not deviate significantly between each film. The film however, with the most irregular Seebeck coefficient pattern is shown for the propylene carbonate and propylene glycol films. In the case of propylene glycol there was no increase in 
Seebeck coefficient across the measured temperature and for propylene carbonate having lowest Seebeck coefficient for any film across most of the temperature range. As shown in Figure 4.7.1 (a) (b) the distinct spectra that the propylene glycol and propylene carbonate films have is significantly different from the both the (thermally assisted) and (non-thermally assisted) hopping films. In conjunction with the corresponding irregular Seebeck coefficient. This infers that the solvent may act on the film in such a way to remove some of the PEDOT regions of the film. 

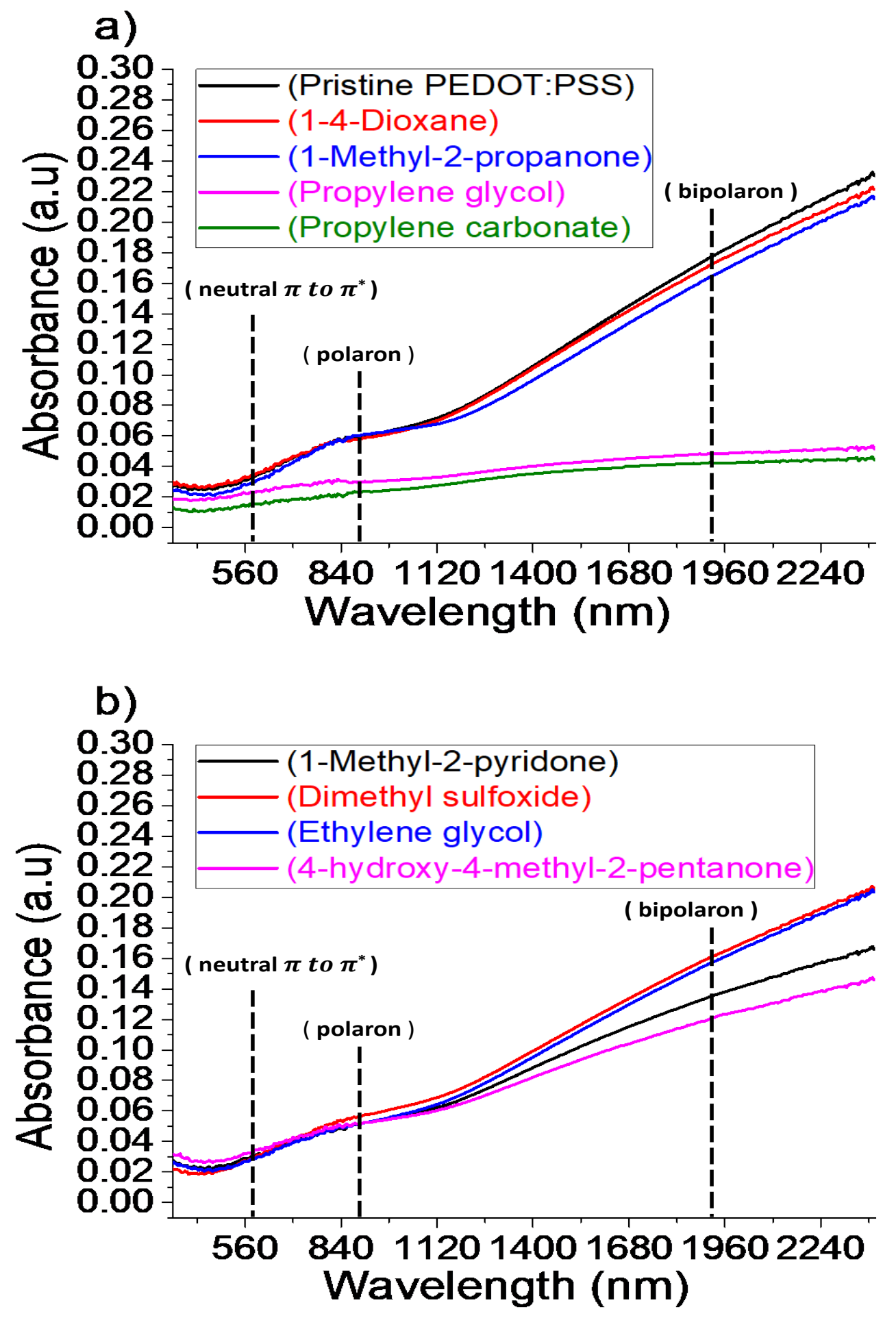

Figure 4.7.1 depicts the UV-Vis-NIR spectra in the range (300-2400 nm) for (a), (thermally assisted hopping,) and (b) (non-thermally assisted hopping) films as well as showing the different molecular state (neutral, polaron, and bipolaron) of the polythiophene indicating majority charger carriers.

As expressed in Figure 4.7.1 (b) are the (non-thermally assisted) hopping mechanism films. Similarly, to the majority of the (thermally assisted) films, the shape $\mathbf{7 8} \mid \mathrm{P}$ a g e 
and pattern of the UV-Vis-NIR spectra appear like the pristine PEDOT:PSS. The most notable film that has a spectrum that deviates from other films is the, 4-methyl-4hydroxy-2-pentanone. The film has the lowest bipolaron peak relative to the other films. Interestingly, it would be expected that if the film were reduced that the polaron region or the neutral region would have an increased peak. It appears however, that the polaron and neutral peak are in the same size as the other films. The film that has the second lowest bipolaron peak is the 1-methyl-2-pentanone. As the Seebeck coefficient did not increase, nor did the UV-Vis-NIR suggest that a usual reduction occurred, several hypotheses may ensue. Firstly, it is still possible that the propylene glycol, propylene carbonate, and to some extent the, 4-methyl-4-hydroxy-2-pentanone films were partially reduced in a way that does not affect the polaron region. This remains a weak argument however because the polaron and neutral regions did not show a subsequent increased intensity. The second hypothesis would be that the three solvent treatments with an emphasis on the propylene glycol and propylene carbonate, and to a much less extent the 4-methyl-4-hydroxy-2-pentanone, induced selective removal of PEDOT from the films.

Figure, 4.7.1 depicts the UV-Vis-NIR for PEDOT:PSS, however in reality the absorbance is formulated by interaction of light with polythiophene molecules with no significant PSS interaction at the range measured. Therefore, if you get a reduced intensity for the 4-methyl-4-hydroxy-2-pentanone's bipolaron region without increasing the polaron region then it could suggest selective PEDOT removal, not typically found in literature. If the PEDOT is selectively removed, then it could suggest either PEDOT degradation or dissolution. (Note: if the UV-Vis-NIR were measured below $180 \mathrm{~nm}$ the PSS region could have been compared to other films to see if it remained unaffected while the PEDOT regions 600-1800 are affected), however, the UV-Vis-NIR used in the study did not have detectors to go below $200 \mathrm{~nm}$. In conjunction, the hypothesis is even stronger for the other two films as the films have a decreased intensity across the whole UV-Vis-NIR range measured. The third hypothesis would be a mixture of any and or all the above. To find out which if at all any of the hypotheses is and or are correct Raman and XPS spectra would need to corroborate the UV-Vis-NIR observations. If the propylene glycol or carbonate treatments do indeed selectively remove PEDOT, further studies into PEDOT dissolution or removal from prepared films may allow researchers to control film 
thickness post fabrication in which it could affect transparency, conductivity, or work function etc. ${ }^{359,360}$
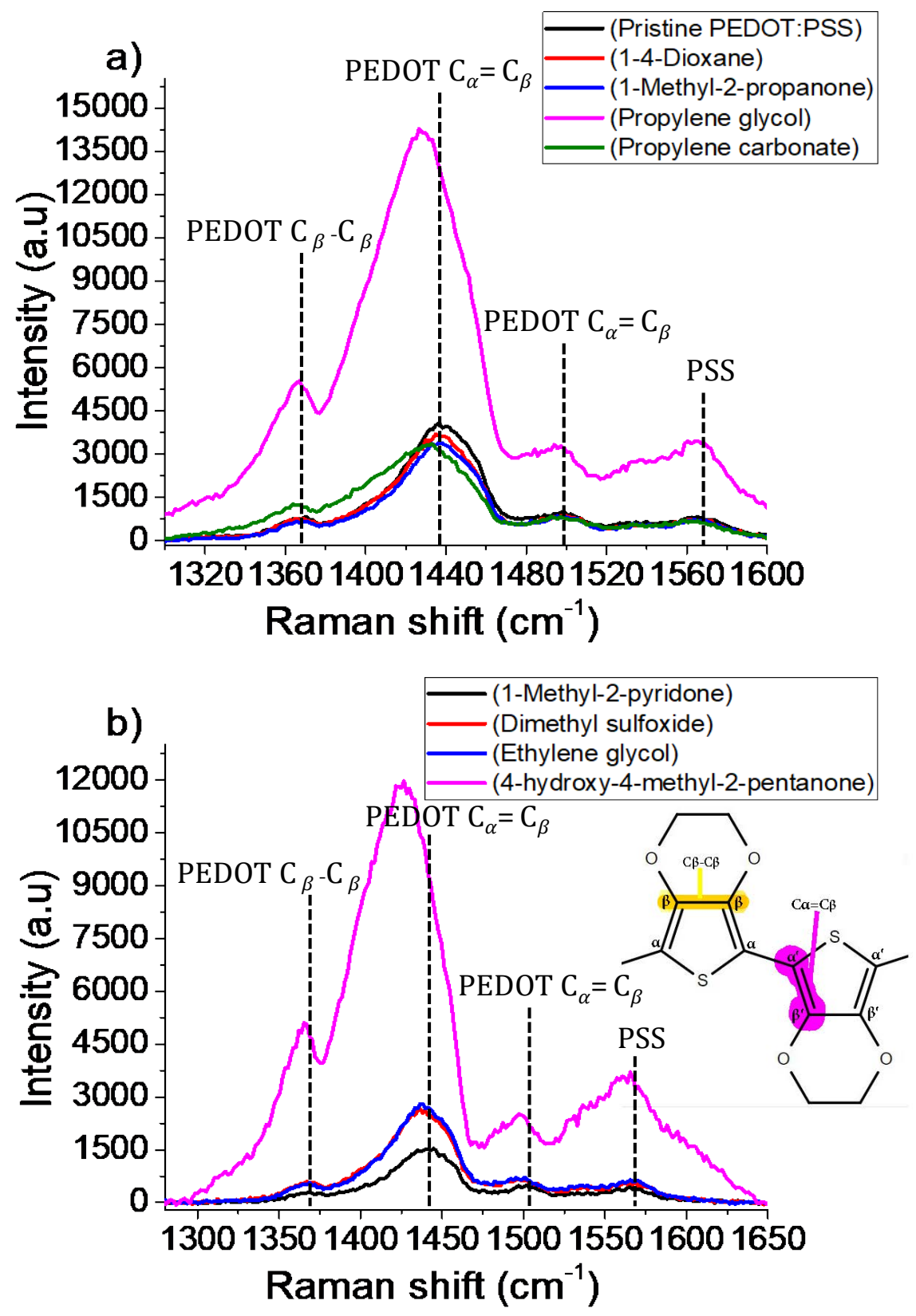

Figure 4.7.3 Raman spectra for the (a) thermally assisted hopping films, and (b) non thermally assisted hopping mechanism films at the area of interest (benzoid to quinoid region).

Present in all films are characteristic peaks and regions that correspond to PEDOT and PSS bond stretching and bending vibrations in respect to Raman spectroscopy. The peak at $1580 \mathrm{~cm}^{-1}$ corresponds to a PSS stretching vibration. The 
one at $1440 \mathrm{~cm}^{-1}$ corresponds to the symmetrical vibrations and finally the peak at $1500 \mathrm{~cm}^{-1}$ depicts the asymmetrical vibrations in PEDOT. Due to the Raman data not being peak fitted or normalized, it is difficult to obtpolyain a quantitative ratio comparison between PEDOT and PSS's region intensity in the films. It is however possible to determine if there is a structural change from benzoid to quinoid in the polythiophene molecular orientation by analysing Raman shift at $1440 \mathrm{~cm}^{-1}$. The quinoid structure is dominated with a more planer structural orientation that allows for better PEDOT stacking therefore it usually denotes improved carrier mobility.

The benzoid form as depicted in in Figure 4.6 .3 (a) expresses the usual conformation of polythiophenes before further treatment post polymer synthesis. The bonds between each monomer are single covalent bonds. In comparison to Figure 4.6.3 (a) the quinoid conformation whereby the monomers are joined by a double bond, the benzoid conformation usually (but not always) suggests a material with lower electrical conductivity. As explained by some researchers, the quinoid conformation may allow for better intra chain charge transport (within a PEDOT chain). ${ }^{298,361,362}$ One particular reason pertains to the fact that in the quinoid conformation, each monomer unit has restricted movement due to monomers being unable to twist with respect to each other due to the double bond, unlike in the single bond benzoid conformation where rotation is only limited by steric hinderances. ${ }^{298,300}$ The quinoid structure is therefore indicative of a PEDOT orientation whereby it's chains are more linear than the benzoid form thereby leading to better $\pi-\pi$ stacking which in turn results in improved crystallinity, thus allowing for a faster charge transport in the chains (please refer to Figure 4.6.2). Another explanation for improved electrical conductivity is explained by a different mechanism in which the selective removal of nonconductive PSS over PEDOT is indicated by a change in the XPS peak ratio between PEDOT and PSS whereby more PEDOT is present at the surface of the film relative to a control (pristine PEDOT:PSS). ${ }^{276,363}$

As shown in Figure 4.7.3 (a) the most notable significant peak intensity at $\left(1440 \mathrm{~cm}^{-1}\right)$ is shown to be produced by the propylene glycol. Although not normalized, it would be expected since all films were measured at the same time frame under the same settings for the intensity to be similarly matched unless a significant chemical change had occurred. It is however not possible to make any meaningful quantification determinations without peak fitting; however, qualitative arguments can 
be made for both extreme differences in intensity and for the chemical shifts. An increased intensity and narrowing of the peak at the $\mathrm{C}_{\alpha}=\mathrm{C}_{\beta}$ position $\left(1440 \mathrm{~cm}^{-1}\right)$ as explained in literature has been linked to dedoping (reduction of the polaron charge on the thiophene rings). The increased intensity and peak narrowing can also be seen at the $\mathrm{C}_{\alpha}=\mathrm{C}_{\beta}$ asymmetric peak at around $1500 \mathrm{~cm}^{-1} \cdot 321,363,364$ In this case however the PSS component has also increased intensity and the peaks have not narrowed relative to other films. The increased PSS Raman peak infer a chemical change to the sulphonic acid groups or increased quantity of PSS. Therefore it less likely that the reason for the observed narrowing and intensified peaks on the symmetrical and asymmetrical $\mathrm{C}_{\alpha}=\mathrm{C}_{\beta}$ is just from the dedoping. This makes it difficult to know if there truly is a dedoping in the case for propylene glycol using Raman spectroscopy alone. (XPS will confirm if dedoping occurred). The spectra for Raman pertaining to propylene glycol does not invalidate the other hypotheses such as PEDOT dedoping. Even if it would be expected for a film with lower PEDOT component to have a lower intensity it's also possible that if the film has also undergone a benzoid to quinoid shift that it would ultimately yield a film with less PSS shielding as the PEDOT chains would adopt a less coiled and a more expanded linear conformation thereby leading to a higher signal even if there is less quantity. Thus, being shielded to a lower extend may cause an increased sensitivity to Raman excitation.

As shown in Figure 4.7.3 (a) at $1440 \mathrm{~cm}^{-1}$ whereby the peak for PEDOT's, benzoid to quinoid shift can be detected whereby a shift below $1434 \mathrm{~cm}^{-1}$ may indicate quinoid vibrational frequency. ${ }^{365} \mathrm{~A}$ significantly strong chemical shift (red-shift) is observed for the propylene glycol film. When compared to the other (thermally assisted mechanism) films as well as the (non-thermally assisted mechanism) films in Figure 4.7.3 (b), it is evident that only propylene glycol and 4-methyl-4-hydroxy-2pentanone showed such an extent of a benzoid to quinoid shift. As shown in Table 4.7.1 the pristine PEDOT:PSS acts as a control. If all films exhibited a benzoid to quinoid shift, then it would be evident with pristine PEDOT:PSS having the most blueshifted wavelength. However, it appears that the 1-methyl-2-pyridone film has a slightly more blue shifted wavelength than pristine PEDOT:PSS and all other films in the study. Interestingly, the 1-methyl-2-pyridone treated film produced the highest electrical conductivity out of all the films in the study. Although it's possible the carrier mobility for this film was also improved via a different mechanism than a 
benzoid to quinoid shift, at the very least due to the Raman shift it the evidence suggests that the film did not have a significant amount of polythiophenes to reorient themselves into a quinoid conformation. Therefore, another mechanism would be required to explain the extremely high electrical conductivity $\left(1100 \mathrm{~S} \mathrm{~cm}^{-1}\right)$ at $40{ }^{\circ} \mathrm{C}$ as shown in Figure 4.5.1 for the sample. As shown in Figure 4.7.3, the intensity of the film does not rise above most films thereby indicating no reduction has taken place. A plausible explanation for the improved conductivity may be a removal of PSS in which the XPS analysis would corroborate. It is also possible that the unique structure of the solvent induces a different mechanism in which further studies would need be conducted. As shown in table 4.7.1 the remaining films showed a red shift with a wavelength higher in energy than pristine PEDOT:PSS. As all films showed an improved electrical conductivity the Raman for these films express that at least partially the reason for the improvement is due to improved carrier mobility via benzoid to quinoid reorientation. As is evident for the dimethyl sulfoxide, ethylene glycol, and 1-methyl-2-propanone the Raman shift is only slight and consistent with literature.

Table 4.7.1 Raman shift of solvent treated films and the control, pristine PEDOT:PSS, at the symmetrical $\mathrm{C}_{\alpha}=\mathrm{C}_{\beta}$ vibration where a benzoid to quinoid shift can be observed with a red shift.

\begin{tabular}{|l|l|}
\hline Film & $\begin{array}{l}\text { Raman shift at benzoid to quinoid region } \\
\left(\text { symmetrical } \mathrm{C}_{\alpha}=\mathrm{C}_{\beta} \text { vibration }\right)\left(\mathrm{cm}^{-1}\right)\end{array}$ \\
\hline pristine PEDOT:PSS & 1440 \\
\hline 1-4-dioxane & 1434 \\
\hline 1-methyl-2-propanone & 1435 \\
\hline 1-methyl-2-pyridone & 1441 \\
\hline dimethyl sulfoxide & 1435 \\
\hline ethylene glycol & 1435 \\
\hline 4-hydroxy-4-methyl-2-pentanone & 1421 \\
\hline propylene glycol & 1426 \\
\hline propylene carbonate & 1432 \\
\hline
\end{tabular}




\subsection{XPS analysis of the PEDOT to PSS ratio}

In literature, it has been shown that XPS can be used to detect the reduction (dedoping) of PEDOT:PSS. If there is reduction taking place on the polythiophene rings, then it would be detectable as a shift towards a lower binding energy (BE) regarding the PEDOT's Sulphur 2p. ${ }^{187,196,298,363}$ It's also been shown that it's possible to tell if PSS, the nonconductive component has been removed from the film and therefore infer some origins behind an improved electrical conductivity. ${ }^{308,324,366}$ This can be done by comparing the ratio of PEDOT's sulphur to PSS's sulphur. This is possible because PEDOT's sulphur and PSS's sulphur are in different chemical environment therefore an XPS measurement can differentiate between them. Figure 4.8.1 depicts the high-resolution Sulphur $2 \mathrm{p}$ Spin-orbital splitting for the pristine PEDOT:PSS. As can be seen, PEDOT's Sulphur (S), has a peak referred to as the $3 / 2$ at $163.9 \mathrm{eV}$ meanwhile the $\frac{1}{2}$ peak is at $165 \mathrm{eV}$. The PSS's S $2 \mathrm{p}$ peak is at a higher BE than PEDOT. PSS can be modelled with only one S Spin-orbital splitting however in this cause (during the modelling with casa XPS software the residual standard deviation was over 2 meaning there was sufficient evidence that the area was based on another Spin orbital splitting not accounted for). ${ }^{196}$ As can be seen the $3 / 2$ peak labelled $\mathrm{PSS}^{-}$is at $168 \mathrm{eV}$ meanwhile the corresponding $1 / 2$ peak is at around $169.23 \mathrm{eV}$. The PSS $^{-}$is bonded either to the residue of $\mathrm{Na}^{+}$or $\mathrm{PEDOT}^{+}$, in which both have lower electrical negativity than the $\mathrm{H}^{+}$. The $3 / 2$ and $\frac{1 / 2}{2}$ peak at $168.4 \mathrm{eV}$ and $169.6 \mathrm{eV}$ respectively correspond to the PSSH. (please refer to Figure 4.6.1). ${ }^{187,196}$

Table 4.8.1 depicts the positions (BE) of each peak shown in Figure 4.8.1 for all films. The change in binding energy $(\triangle \mathrm{BE})$, is also shown, at the furthest column to the right. This column depicts if there has been reduction or oxidation on the thiophene backbone by analysing the change in chemical environment corresponding to PEDOT's S 2 $p_{3 / 2}$ region. The number is formulated by taking PEDOT's BE at the S $2 p_{\text {(3/2) }}$ peak away from the same peak of other films. If the number obtained is positive and larger than 0 then it indicates reduction towards a lower, BE. If the number is negative, then it indicates oxidation. This can infer different things such as bonding changes. In this analysis it will be focused on inferences of reduction or oxidation. As shown in Figure 4.8.2 is the quantified molecular ratio (\%) between PEDOT and PSS for all the 9 films. When analysing both figure 4.8.2 and Table 4.8.1 the pristine 
PEDOT:PSS film has a PEDOT concentration of $44.37 \%$ (please note that the model used to obtain this concentration included the tail towards higher binding energy present in oxidised PEDOT). It is usually seen if removal of PSS is the primary cause of improved electrical conductivity for there to be a substantial $(5 \%)$ or more increase in the amount of PEDOT present in the film relative to PSS. In this case the three films with the highest electrical conductivity was the ethylene glycol, the DMSO, and the 1methyl-2-pyridone films.

The ethylene glycol had the highest PEDOT quantity (46.61\%), meanwhile the 1-methyl-2-pyridone, had $45.22 \%$ and the DMSO had $44.31 \%$. Although not by a substantial amount, the two films with more PEDOT concentration than the pristine PEDOT:PSS film indicates that at least some part of the improvement of the electrical conductivity is linked to the selective removal of PSS from the film. Because the DMSO film was the second most conductive film yet it showed a similar PEDOT concentration to the pristine PEDOT:PSS film, it suggests that other mechanisms were responsible for the improved electrical conductivity such as reorientation of polythiophenes from benzoid to quinoid. Several XPS spectra were taken at different sample locations and for all samples the quantified data is consistent as there was little variation within each sample. It is however, not enough to place the explanation for conductivity improvement regarding the DMSO film on the change from benzoid to quinoid alone because even the films with lower electrical conductivity showed a benzoid to quinoid shift. In conjunction with a recent paper concerning charge transport in which it was argued that charge transport in PEDOT:PSS operates via fast and slow mechanisms in which the solvent induced morphological change may improve grain morphology (detected by AFM) of PEDOT chains to what has been termed as fast transport. ${ }^{361}$ It is therefore thought a morphological change occurred not detected by the optical microscopy is probably a more significant reason for the DMSO's improved conductivity. 


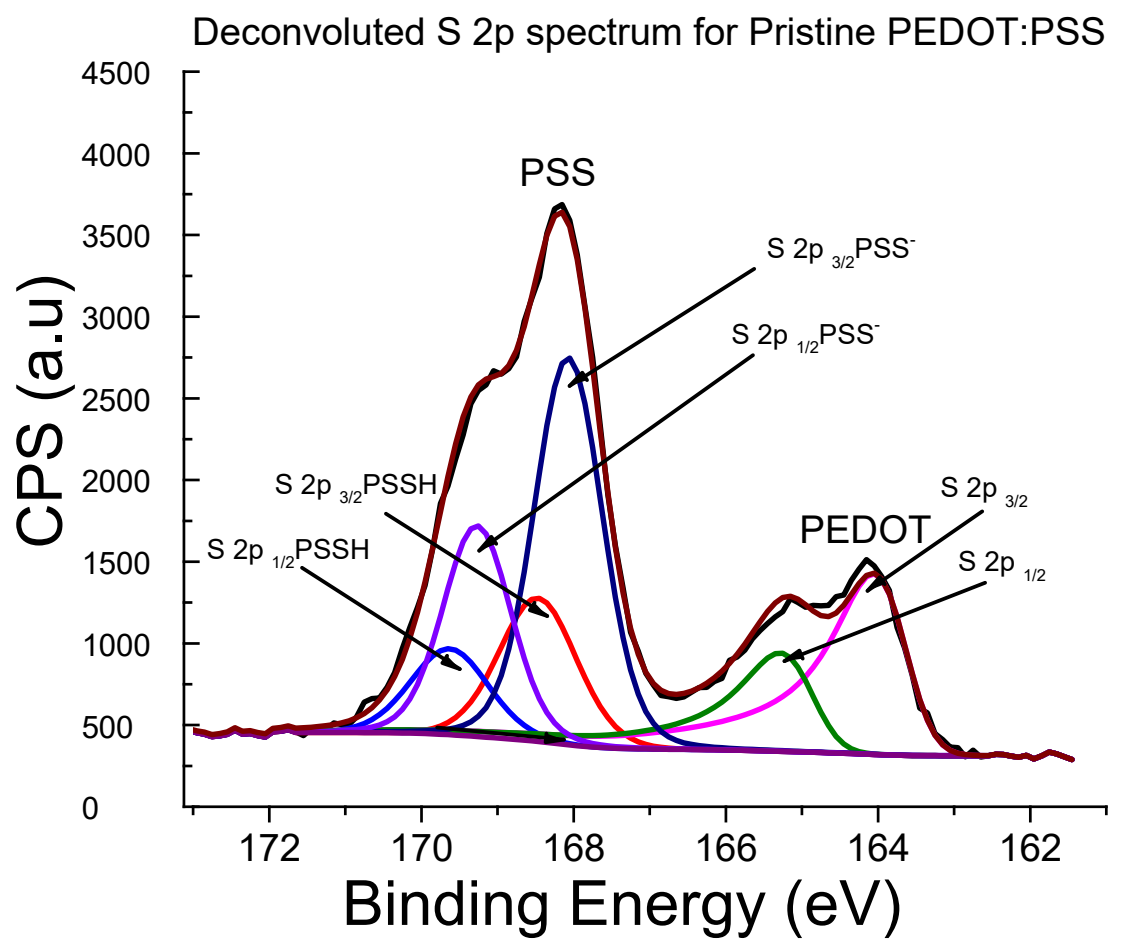

Figure 4.8.1 depicting the Sulphur $2 p(3 / 2)$ and (1/2) Spin-orbital splitting for PEDOT and PSS. The PEDOT region is centred around $164 \mathrm{eV}$ meanwhile the PSS region is around $169 \mathrm{eV}$.

Throughout the research study it was hard to detect relevant PEDOT diffraction peaks using XRD and make meaningful interpretation of the almost always noisy signals therefore non were conducted during this study. More studies would need to be taken to understand the true origin of the improved electrical conductivity. The results however indicate that the improved electrical conductivity via internal treatment of solvent is more complex than just removal of PSS or benzoid to quinoid shift.

A hypothesis was made in chapter 4.7 based on the reduced bipolaron and polaron intensity that the propylene carbonate films have either been reduced and or undergone “selective PEDOT" removal. If One analyses Figure 4.8.2 and looks at Table 4.8.1 it is shown that the PEDOT \% for both films are lower than the pristine PEDOT:PSS films PEDOT \%. The propylene glycol had a PEDOT \% of 40.88 in which compared to the pristine PEDOT:PSS's $44.61 \%$ PEDOT concentration shows a clear reduction in the amount of PEDOT in the film. This is a clear evidence for the hypothesis. The propylene carbonate however did not have its PEDOT \% (43.16) reduced by a substantial amount to make a stronger case for it being the reason for the observed (low absorption on the UV-VIS). Still, it was clear that something unexplained was 
responsible for the appearance of the UV-Vis spectrum being different from other films. Looking at Table 4.8.1 it is shown that the $\Delta \mathrm{BE}$ for propylene carbonate is the most significant $(0.46 \mathrm{eV})$. This is a substantial shift and in conjunction with the low $\mathrm{UV}-\mathrm{V}$ is intensity and the quantification showing reduced PEDOT, gives support to the hypothesis that it was both reduced and PEDOT was selectively removed. With The exception for the 1-4-dioxane film $\Delta \mathrm{BE}-0.08 \mathrm{eV}$, all films have a shift at $0.06 \mathrm{eV}$ or smaller. The small shift is not significant enough to give strong evidence of reduction however that is what the data suggests. The negative $\Delta \mathrm{BE}$ for the 1-4-dioxane suggests that the film was slightly oxidised.

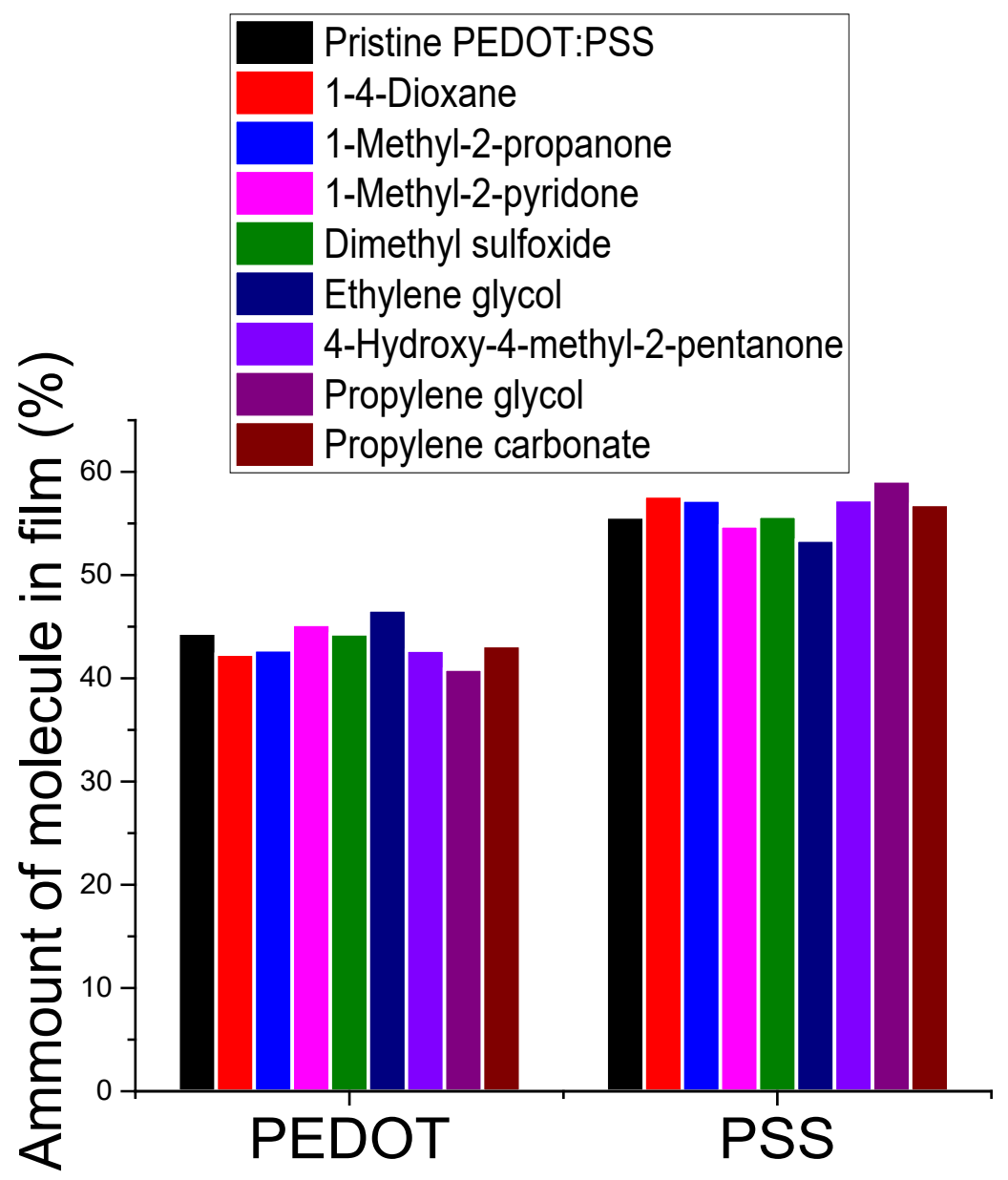

Figure 4.8.2 the molecular percentage ratio between PEDOT and PSS for the 9 films. 
$\mathbf{8 8} \mid \mathrm{P}$ a g e 
Table 4.8.1 quantified data for all films and the relative position of each sub peak component for the S $2 p(3 / 2)$ and (1/2) Spin orbital split

\begin{tabular}{|c|c|c|c|c|c|c|c|}
\hline Film & $\begin{array}{l}\text { Name } \\
\text { of peak }\end{array}$ & $\begin{array}{l}\mathrm{BE} \text { of } \\
\text { position } \\
(\mathrm{eV})\end{array}$ & $\begin{array}{l}\text { concentrat } \\
\text { ion at the } \\
\text { region } \\
(\%)\end{array}$ & $\begin{array}{l}\text { Total } \\
\text { PSS } \\
\text { concentr } \\
\text { ation } \\
(\%)\end{array}$ & $\begin{array}{l}\text { Total PEDOT } \\
\text { concentration } \\
(\%)\end{array}$ & $\begin{array}{l}\text { PEDOT } \\
\text { concentra } \\
\text { tion/PSS } \\
\text { concentra } \\
\text { tion }\end{array}$ & $\begin{array}{l}\triangle B E=\mathrm{BE} \text { of } \\
\text { pristine } \\
\text { PEDOT:PSS- } \\
\mathrm{BE} \text { of solvent } \\
\text { treatment for } \\
\text { the Sulphur } 2 \mathrm{p} \\
3 / 2 \text { peak) }(\mathrm{eV})\end{array}$ \\
\hline \multirow{6}{*}{$\begin{array}{l}\text { 4- } \\
\text { hydroxy- } \\
\text { 4-methyl- } \\
2- \\
\text { pentanone }\end{array}$} & $\begin{array}{l}\mathrm{S} \\
2 \mathrm{p} 3 / 2 \\
\mathrm{PSSH}\end{array}$ & 168.49 & 19.78 & 57.29 & 42.72 & 0.74 & 0 \\
\hline & $\begin{array}{l}\mathrm{S} \\
2 \mathrm{p} 1 / 2 \\
\mathrm{PSSH}\end{array}$ & 169.65 & 11.87 & & & & \\
\hline & $\begin{array}{l}\mathrm{S} \\
2 \mathrm{p} 3 / 2 \\
\mathrm{PEDO} \\
\mathrm{T}\end{array}$ & 163.89 & 28.48 & & & -- & \\
\hline & $\begin{array}{l}\mathrm{S} \\
2 \mathrm{p} 1 / 2 \\
\mathrm{PEDO} \\
\mathrm{T}\end{array}$ & 165.12 & 14.24 & & & -- & \\
\hline & $\begin{array}{l}\text { S } 2 p \\
3 / 2 \\
\text { PSS- }\end{array}$ & 168.09 & 16.6 & & & -- & \\
\hline & $\begin{array}{l}\quad 2 p \\
1 / 2 \\
\text { PSS- }\end{array}$ & 169.29 & 9.04 & & & -- & \\
\hline \multirow[t]{4}{*}{$\begin{array}{l}\text { propylene } \\
\text { carbonate }\end{array}$} & $\begin{array}{l}\mathrm{S} \\
2 \mathrm{p} 3 / 2 \\
\mathrm{PSSH}\end{array}$ & 169.19 & 13.99 & 56.84 & 43.16 & 0.75 & 0.46 \\
\hline & $\begin{array}{l}\mathrm{S} \\
2 \mathrm{p} 1 / 2 \\
\mathrm{PSSH}\end{array}$ & 170.39 & 8.4 & & & -- & \\
\hline & $\begin{array}{l}\mathrm{S} \\
2 \mathrm{p} 3 / 2 \\
\mathrm{PEDO} \\
\mathrm{T}\end{array}$ & 163.44 & 27.95 & & & -- & \\
\hline & $\begin{array}{l}\mathrm{S} \\
2 \mathrm{p} 1 / 2\end{array}$ & 164.67 & 15.21 & & & -- & \\
\hline
\end{tabular}

$\mathbf{8 9} \mid \mathrm{P}$ a g e 


\begin{tabular}{|c|c|c|c|c|c|c|c|}
\hline & $\begin{array}{l}\text { PEDO } \\
\mathrm{T}\end{array}$ & & & & & & \\
\hline & $\begin{array}{l}\text { S 2p } \\
3 / 2 \\
\text { PSS- }\end{array}$ & 168.18 & 22.97 & & & -- & \\
\hline & $\begin{array}{l}\text { S } 2 p \\
1 / 2 \\
\text { PSS- }\end{array}$ & 169.38 & 11.48 & & & -- & \\
\hline \multirow[t]{6}{*}{$\begin{array}{l}\text { 1-methyl- } \\
2- \\
\text { pyridone }\end{array}$} & $\begin{array}{l}\mathrm{S} \\
2 \mathrm{p} 3 / 2 \\
\mathrm{PSSH}\end{array}$ & 168.46 & 17.72 & 54.77 & 45.22 & 0.82 & 0.04 \\
\hline & $\begin{array}{l}\mathrm{S} \\
2 \mathrm{p} 1 / 2 \\
\mathrm{PSSH}\end{array}$ & 169.62 & 10.63 & & & -- & \\
\hline & $\begin{array}{l}\mathrm{S} \\
2 \mathrm{p} 3 / 2 \\
\mathrm{PEDO} \\
\mathrm{T}\end{array}$ & 163.86 & 30.15 & & & -- & \\
\hline & $\begin{array}{l}\mathrm{S} \\
2 \mathrm{p} 1 / 2 \\
\text { PEDO } \\
\mathrm{T}\end{array}$ & 165.08 & 15.07 & & & -- & \\
\hline & $\begin{array}{l}\text { S 2p } \\
3 / 2 \\
\text { PSS- }\end{array}$ & 168.06 & 17.11 & & & -- & \\
\hline & $\begin{array}{l}\text { S } 2 p \\
1 / 2 \\
\text { PSS- }\end{array}$ & 169.26 & 9.31 & & & -- & \\
\hline \multirow[t]{4}{*}{$\begin{array}{l}\text { dimethyl } \\
\text { sulfoxide }\end{array}$} & $\begin{array}{l}\mathrm{S} \\
2 \mathrm{p} 3 / 2 \\
\mathrm{PSSH}\end{array}$ & 168.4 & 22.86 & 55.69 & 44.31 & 0.79 & 0.01 \\
\hline & $\begin{array}{l}\mathrm{S} \\
2 \mathrm{p} 1 / 2 \\
\mathrm{PSSH}\end{array}$ & 169.56 & 13.72 & & & -- & \\
\hline & $\begin{array}{l}\mathrm{S} \\
2 \mathrm{p} 3 / 2 \\
\mathrm{PEDO} \\
\mathrm{T}\end{array}$ & 163.89 & 29.54 & & & -- & \\
\hline & $\begin{array}{l}\mathrm{S} \\
2 \mathrm{p} 1 / 2 \\
\text { PEDO } \\
\mathrm{T}\end{array}$ & 165.12 & 14.77 & & & -- & \\
\hline
\end{tabular}

90|P a g e 


\begin{tabular}{|c|c|c|c|c|c|c|c|}
\hline & \begin{tabular}{|lr}
$\mathrm{S} \quad 2 \mathrm{p}$ \\
$3 / 2$ \\
PSS-
\end{tabular} & 168 & 12.74 & & & -- & \\
\hline & \begin{tabular}{|lr} 
S $\quad 2 p$ \\
$1 / 2$ \\
PSS-
\end{tabular} & 169.2 & 6.37 & & & -- & \\
\hline \multirow{6}{*}{$\begin{array}{l}\text { 1-methyl- } \\
2- \\
\text { propanon } \\
\text { e }\end{array}$} & \begin{tabular}{|l|}
$\mathrm{S}$ \\
$2 \mathrm{p} 3 / 2$ \\
$\mathrm{PSSH}$
\end{tabular} & 168.51 & 20.21 & 57.27 & 42.75 & 0.74 & 0.06 \\
\hline & \begin{tabular}{|l}
$\mathrm{S}$ \\
$2 \mathrm{p} 1 / 2$ \\
$\mathrm{PSSH}$
\end{tabular} & 169.67 & 12.13 & & & -- & \\
\hline & \begin{tabular}{|l|}
$\mathrm{S}$ \\
$2 \mathrm{p} 3 / 2$ \\
$\mathrm{PEDO}$ \\
$\mathrm{T}$
\end{tabular} & 163.84 & 27.68 & & & -- & \\
\hline & \begin{tabular}{|l|}
$\mathrm{S}$ \\
$2 \mathrm{p} 1 / 2$ \\
$\mathrm{PEDO}$ \\
$\mathrm{T}$
\end{tabular} & 165.07 & 15.07 & & & -- & \\
\hline & \begin{tabular}{|l|}
$\mathrm{S} \quad 2 \mathrm{p}$ \\
$3 / 2$ \\
PSS-
\end{tabular} & 168.11 & 16.14 & & & -- & \\
\hline & \begin{tabular}{|l} 
S $2 p$ \\
$1 / 2$ \\
PSS-
\end{tabular} & 169.31 & 8.79 & & & -- & \\
\hline \multirow[t]{4}{*}{$\begin{array}{l}\text { ethylene } \\
\text { glycol }\end{array}$} & \begin{tabular}{|l}
$\mathrm{S}$ \\
$2 \mathrm{p} 3 / 2$ \\
$\mathrm{PSSH}$
\end{tabular} & 168.49 & 15.61 & 53.39 & 46.61 & 0.87 & 0.06 \\
\hline & \begin{tabular}{|l}
$\mathrm{S}$ \\
$2 \mathrm{p} 1 / 2$ \\
$\mathrm{PSSH}$
\end{tabular} & 169.65 & 9.36 & & & -- & \\
\hline & \begin{tabular}{|l|}
$\mathrm{S}$ \\
$2 \mathrm{p} 3 / 2$ \\
$\mathrm{PEDO}$ \\
$\mathrm{T}$
\end{tabular} & 163.84 & 30.18 & & & -- & \\
\hline & \begin{tabular}{|l}
$\mathrm{S}$ \\
$2 \mathrm{p} 1 / 2$ \\
$\mathrm{PEDO}$ \\
$\mathrm{T}$
\end{tabular} & 165.07 & 16.43 & & & -- & \\
\hline
\end{tabular}

91| P a g e 


\begin{tabular}{|c|c|c|c|c|c|c|c|}
\hline & $\begin{array}{l}\text { S } 2 p \\
3 / 2 \\
\text { PSS- }\end{array}$ & 168.09 & 18.4 & & & -- & \\
\hline & $\begin{array}{l}\text { S 2p } \\
1 / 2 \\
\text { PSS- }\end{array}$ & 169.29 & 10.02 & & & -- & \\
\hline \multirow[t]{6}{*}{$\begin{array}{l}\text { propylene } \\
\text { glycol }\end{array}$} & $\begin{array}{l}\mathrm{S} \\
2 \mathrm{p} 3 / 2 \\
\mathrm{PSSH}\end{array}$ & 168.45 & 24.14 & 59.11 & 40.88 & 0.69 & 0.06 \\
\hline & $\begin{array}{l}\mathrm{S} \\
2 \mathrm{p} 1 / 2 \\
\mathrm{PSSH}\end{array}$ & 169.61 & 14.48 & & & -- & \\
\hline & $\begin{array}{l}\mathrm{S} \\
2 \mathrm{p} 3 / 2 \\
\mathrm{PEDO} \\
\mathrm{T}\end{array}$ & 163.84 & 26.47 & & & -- & \\
\hline & $\begin{array}{l}\mathrm{S} \\
2 \mathrm{p} 1 / 2 \\
\mathrm{PEDO} \\
\mathrm{T}\end{array}$ & 165.06 & 14.41 & & & -- & \\
\hline & $\begin{array}{l}\text { S 2p } \\
3 / 2 \\
\text { PSS- }\end{array}$ & 168.05 & 13.27 & & & -- & \\
\hline & $\begin{array}{l}\text { S } 2 p \\
1 / 2 \\
\text { PSS- }\end{array}$ & 169.25 & 7.22 & & & -- & \\
\hline \multirow[t]{5}{*}{$\begin{array}{l}1-4- \\
\text { dioxane }\end{array}$} & $\begin{array}{l}\mathrm{S} \\
2 \mathrm{p} 3 / 2 \\
\mathrm{PSSH}\end{array}$ & 168.47 & 20.25 & 57.67 & 42.33 & 0.73 & -0.08 \\
\hline & $\begin{array}{l}\mathrm{S} \\
2 \mathrm{p} 1 / 2 \\
\mathrm{PSSH}\end{array}$ & 169.63 & 12.15 & & & -- & \\
\hline & $\begin{array}{l}\mathrm{S} \\
2 \mathrm{p} 3 / 2 \\
\text { PEDO } \\
\mathrm{T}\end{array}$ & 163.98 & 28.22 & & & -- & \\
\hline & $\begin{array}{l}\mathrm{S} \\
2 \mathrm{p} 1 / 2 \\
\mathrm{PEDO} \\
\mathrm{T}\end{array}$ & 165.21 & 14.11 & & & -- & \\
\hline & $\begin{array}{l}\text { S } 2 p \\
3 / 2 \\
\text { PSS- }\end{array}$ & 168.07 & 16.36 & & & -- & \\
\hline
\end{tabular}




\begin{tabular}{|c|c|c|c|c|c|c|c|}
\hline & $\begin{array}{l}\text { S } 2 p \\
1 / 2 \\
\text { PSS- }\end{array}$ & 169.27 & 8.91 & & & -- & \\
\hline \multirow[t]{6}{*}{$\begin{array}{l}\text { pristine } \\
\text { PEDOT:P } \\
\text { SS }\end{array}$} & $\begin{array}{l}\mathrm{S} \\
2 \mathrm{p} 3 / 2 \\
\mathrm{PSSH}\end{array}$ & 168.45 & 19.47 & 55.62 & 44.37 & 0.79 & 0 \\
\hline & $\begin{array}{l}\mathrm{S} \\
2 \mathrm{p} 1 / 2 \\
\mathrm{PSSH}\end{array}$ & 169.61 & 11.68 & & & -- & \\
\hline & $\begin{array}{l}\mathrm{S} \\
2 \mathrm{p} 3 / 2 \\
\mathrm{PEDO} \\
\mathrm{T}\end{array}$ & 163.9 & 28.73 & & & -- & \\
\hline & $\begin{array}{l}\mathrm{S} \\
2 \mathrm{p} 1 / 2 \\
\mathrm{PEDO} \\
\mathrm{T}\end{array}$ & 165.08 & 15.64 & & & -- & \\
\hline & $\begin{array}{l}\text { S } 2 p \\
3 / 2 \\
\text { PSS- }\end{array}$ & 168.05 & 15.84 & & & -- & \\
\hline & $\begin{array}{l}\text { S } 2 p \\
1 / 2 \\
\text { PSS- }\end{array}$ & 169.25 & 8.63 & & & -- & \\
\hline
\end{tabular}

\subsection{Conclusion}

All films that were treated with a polar solvent exhibited an improved electrical conductivity. The DMSO, as well as propylene glycol, propylene carbonate and 4hydroxy-4-methyl-2propanone, could be linked to a benzoid to quinoid structural change to explain some of the improved conductivity as depicted by the Raman spectra. According to the UV-Vis data it suggests that most films were heavily doped (bipolaronic) except for, propylene glycol, and propylene carbonate. The inference here is that propylene glycol and propylene carbonate can be explored further to observe effects it has on PEDOT:PSS as potential solvents or chemical agents to study PEDOT degradation mechanisms. The propylene glycol film exhibited the most significant PEDOT removal. For the 1-methyl-2-pyrrolidinone which exhibited the highest conductivity meanwhile having no benzoid to quinoid structural change must be explored further to elucidate mechanics behind electrical conductivity 
improvement. The XPS showed it has slightly increased PEDOT \% than the pristine PEDOT:PSS film but by a negligible factor therefore not being a viable reason to explain the improvement. It is possible that the improvement would be detected by XRD or AFM. 


\section{Chapter 5 Enhanced Electrical Conductivity and Seebeck Coefficient in PEDOT:PSS via a Two-Step Ionic liquid and NaBH4 Treatment.}

\subsection{Introduction}

As shown in literature it is possible to significantly increase the electrical conductivity of PEDOT:PSS via treatment with polar solvents, acids and salt solutions. ${ }^{128,209}$ Studies have yielded conductivities of 620, 640, 800, 1300 and $1900 \mathrm{~S}$ $\mathrm{cm}^{-1}$ by treating PEDOT:PSS with dimethyl sulfoxide (DMSO), ethylene glycol, polyethylene glycol, methanol and formic acid, respectively. ${ }^{210,211}$ Solvent treatments, however, do not significantly affect the Seebeck coefficient of PEDOT:PSS as discovered in chapter 4 and seen in literature. ${ }^{315,337}$ As explored in chapter 4 , several solvents were utilized as secondary dopants for PEDOT:PSS. It was discovered that the electrical conductivity improved for all samples treated with a solvent. It was also observed however, that the Seebeck coefficient was not significantly affected by the treatments. Because of this, the PF seen for all films across the temperature profile (40 ${ }^{\circ} \mathrm{C}$ to $>200{ }^{\circ} \mathrm{C}$ ) was higher than the pristine PEDOT:PSS however, less than $33 \mu \mathrm{W}$ $\mathrm{m}^{-1} \mathrm{~K}^{-2}$. It is also evident that the treatment method also has an influence on thermoelectric performance. For example, DMSO (internal treatment) of the PEDOT:PSS suspension yields a PF of $18.2 \mu \mathrm{W} \mathrm{m} \mathrm{m}^{-1} \mathrm{~K}^{-2}$ (as was observed in the PF for films in chapter 4 Figure 4.5.6 (a) at around room temperature). This is contrasted by post treatment of the PEDOT:PSS film by DMSO which gives a PF of $30.1 \mu \mathrm{W}$ $\mathrm{m}^{-1} \mathrm{~K}^{-2}$ at around room temperature. ${ }^{212}$

In 2007, the first study to determine the effects of ionic liquid (IL) treatment on the electrical conductivity of PEDOT:PSS was employed by utilizing five different ionic liquids. ${ }^{216}$ In the study, 1-butyl-3-methylimidazolium bromide (BMIM:Br), 1benzyl-3-methylimidazolium chloride (BzMIM:Cl), 1-butyl3-methylimidazolium tetrafluoroborate (BMIM:BF4) and 1-butyl-1-methylpyrrolidium chloride (BMPro: $\mathrm{Cl}$ ) and 1 ethyl-3-methylimidazolium chloride (EMIM:CL) were employed. 
The BMIM:BF 4 treated film achieved the highest conductivity of $136 \mathrm{~S} \mathrm{~cm}^{-1}$. More recently, ${ }^{217}$ significantly higher electrical conductivity has been shown by controlling the molecular ordering in PEDOT:PSS chains by using 1-ethyl-3-methylimidazolium tetracyanoborate (EMIM:TCB), achieving an electrical conductivity of $2103 \mathrm{~S} \mathrm{~cm}^{-1}$. PEDOT:PSS-ionic liquid composites have also exhibited resistance to mechanical deformation while maintaining high performance. ${ }^{218}$ These properties of PEDOT:PSS-ionic liquid composites make them particularly suitable candidates for printing onto flexible substrates and textiles because they can withstand shape deformation that could occur in wearable electronics. It has also been shown that ionic liquid treatments may improve environmental stability of PEDOT:PSS. In a study by Kyaw et al, it was discovered that under harsher conditions $\left(70{ }^{\circ} \mathrm{C}\right.$ and $\left.75 \%\right)$ for over 480 hours that the pristine PEDOT:PSS film lost $77 \%$ of its electrical conductivity meanwhile the ionic liquid films lost only $22 \%$ of the initial electrical conductivity. ${ }^{367}$

Whilst solvent and ionic liquid treatments leave Seebeck coefficient largely unchanged, treatment with base solutions and reducing agents can tune the oxidation state of PEDOT and thus the Seebeck coefficient can be increased. ${ }^{368} \mathrm{NaOH}$ solution treatment was been used to increase the Seebeck coefficient from $12.6 \mu \mathrm{V} \mathrm{K}^{-1}$ to 54 $\mu \mathrm{V} \mathrm{K}^{-1}$ in pristine PEDOT:PSS, however, the treatment lead to a decrease in electrical conductivity from $837 \mathrm{~S} \mathrm{~cm}^{-1}$ to $0.04 \mathrm{~S} \mathrm{~cm}^{-1}$. Only dual solvent/base treatment by mixing a DMSO and $\mathrm{NaOH}$ produced a significantly improved PF reaching $33 \mu \mathrm{W}$ $\mathrm{m}^{-1} \mathrm{~K}^{-2} \cdot{ }^{368}$ Seebeck coefficients of $104 \mu \mathrm{V} \mathrm{K}{ }^{-1}$ have been achieved in $\mathrm{NaBH}_{4}$ treated PEDOT:PSS, but resulted in a reduction of the electrical conductivity from 716 to 11 $\mathrm{S} \mathrm{cm}^{-1}{ }^{139}$ Hydrazine has also been utilized and a PF of $112 \mu \mathrm{W} \mathrm{m} \mathrm{K}^{-1} \mathrm{~K}^{-2}$ has been achieved by treating the PEDOT:PSS with a hydrazine in DMSO solution yielding an electrical conductivity of $578 \mathrm{~S} \mathrm{~cm}^{-1}$ and Seebeck coefficient was $67 \mu \mathrm{V} \mathrm{K}^{-1}{ }^{209}$

To the best of our knowledge, prior to this experimental work there had not been investigations looking at the effects of a reducing agent treatment on a thin film PEDOT:PSS-ionic liquid composite. In this study 1-Ethyl-3-methylimidazolium bis(trifluoromethyl sulfonyl)imide (EMIM:TFSI): PEDOT:PSS composite is synthesized at different concentrations of EMIM:TFSI relative to PEDOT:PSS and the subsequent post treatment with $\mathrm{NaBH}_{4}$-DMSO solution. It was observed in the PEDOT:PSS ionic liquid composite a superior electrical conductivity and unaffected Seebeck coefficient. This gave evidence to suggest that the TFSI anion did not reduce 
the polythiophene chains. The reducing agent $\mathrm{NaBH}_{4}$ is utilized as a post treatment to tune the oxidation state and improve the Seebeck coefficient. This study also explores the mechanisms responsible for the improved electrical conductivity and Seebeck coefficient.

\subsection{Fabrication of PEDOT:PSS EMIM:TFSI Composite Films}

\subsubsection{Preparation of EMIM:TFSI films}

A $1 \mathrm{~mm}$ thick plain nonconductive glass was cut into $2.2 \mathrm{~cm}$ by $2.2 \mathrm{~cm}$ pieces and washed in a Helmanax solution. The glass substrates were then rinsed with deionised water and subsequently rinsed with acetone and then isopropanol. The glass was then dried in a stream of nitrogen gas. The PEDOT:PSS dispersion $(3000 \mu \mathrm{L})$ was placed into $7 \mathrm{~mL}$ glass vials. Aliquots of $15,30,45,60$ and $75 \mu \mathrm{L}$ of 1-Ethyl-3methylimidazoliumbis(trifluoromethyl sulfonyl)imide (EMIM:TFSI) were added to separate vials to give $0.5 \%, 1 \%, 1.5 \%, 2 \%$ and $2.5 \%(\mathrm{v} / \mathrm{v})$ dispersions. The vials were shaken using a vortex mixer then heated at $120{ }^{\circ} \mathrm{C}$ for $3 \mathrm{~min}$. Then $300 \mu \mathrm{L}$ of the PEDOT:PSS/EMIM:TFSI solutions were then pipetted onto a pre-cleaned glass substrate and spin-coated at $2000 \mathrm{rpm}$ with an acceleration speed of $2000 \mathrm{rpm} \cdot \mathrm{s}^{-1}$ for $30 \mathrm{~s}$, then annealed at $120{ }^{\circ} \mathrm{C}$ in air for $10 \mathrm{~min}$. The films produced were $50-70 \mathrm{~nm}$, as determined by profilometry.

\subsubsection{Fabrication of PEDOT:PSS EMIM:TFSI-NaBH ${ }_{4}$ Composite Films}

To make the $1 \%$ (w/v) $\mathrm{NaBH}_{4}$-DMSO reducing agent solution, $20 \mathrm{mg}$ of $\mathrm{NaBH}_{4}$ was dissolved in $2 \mathrm{~mL}$ of DMSO by shaking it using a vortex mixer and heating at 120 ${ }^{\circ} \mathrm{C}$ for $5 \mathrm{~min}$. Then $200 \mu \mathrm{L}$ of the $\mathrm{NaBH}_{4}$ solution was drop cast on to the PEDOT:PSSEMIM:TFSI films at room temperature for $1 \mathrm{~min}$ and then rinsed with acetone. The films were then dried in dry nitrogen gas before annealing at $120^{\circ} \mathrm{C}$ for $5 \mathrm{~min}$. The films thickness remained relatively unchanged after $\mathrm{NaBH}_{4}$ treatment $(55-65 \mathrm{~nm})$. 


\subsection{Results and Discussion}

\subsubsection{Thermoelectric Performance of PEDOT:PSS EMIM:TFSI}

\section{Composite Films}

Figure 5.3.1 shows the thermoelectric properties of PEDOT:PSS and EMIM:TFSI treated PEDOT:PSS films based on the solution $\mathrm{v} / \mathrm{v}$ percentage from which they were produced. 

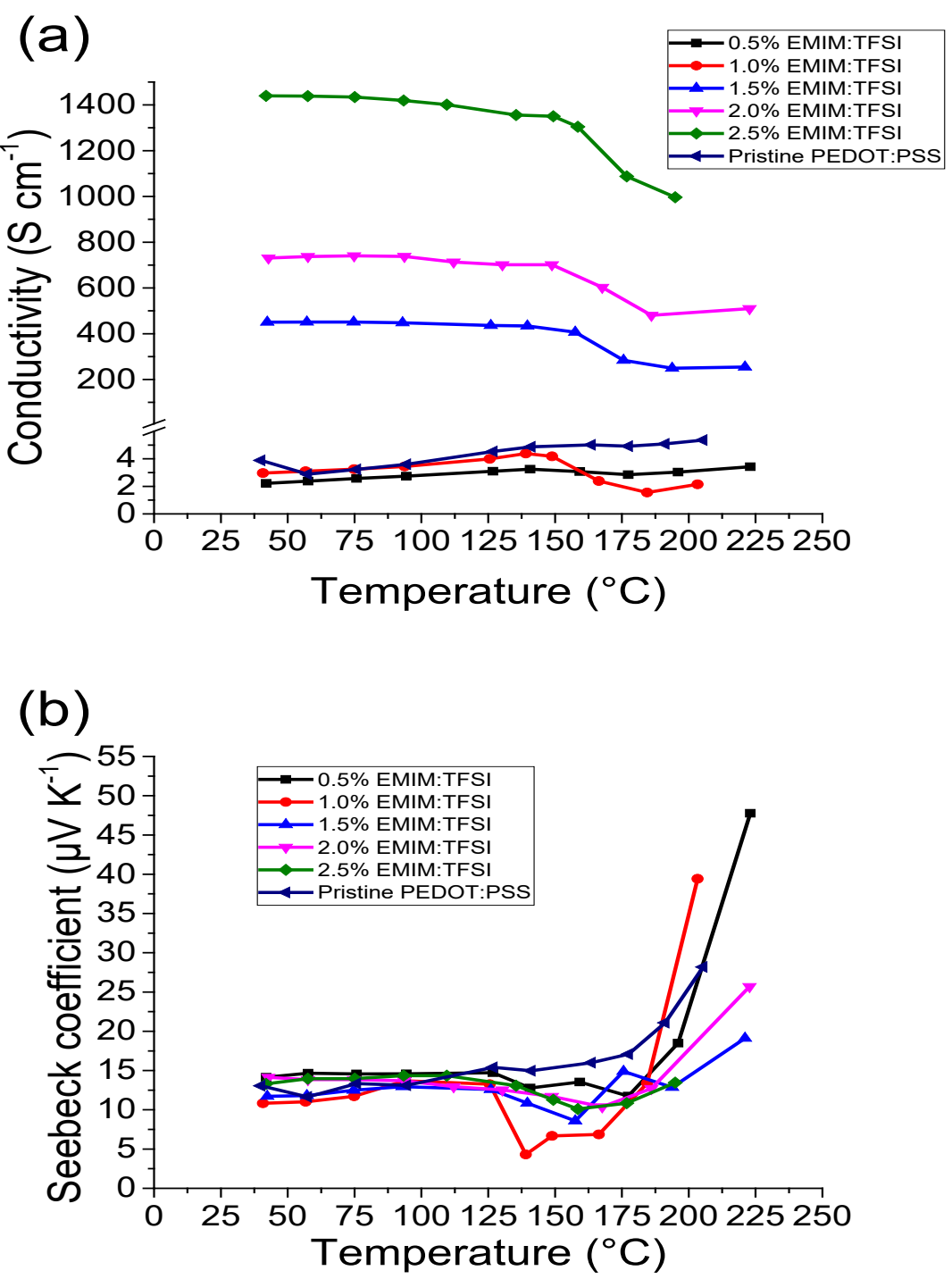

(c)

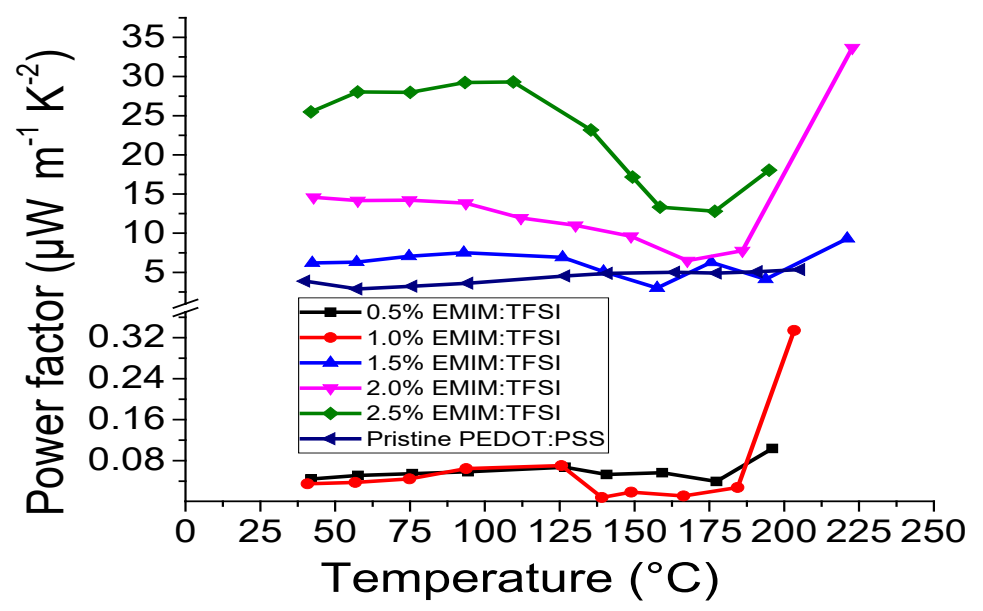

Figure 5.3.1. Thermoelectric properties of 1-Ethyl-3-methylimidazolium bis(trifluoromethylsulfonyl)imide (EMIM:TFSI) treated films, a) the electrical conductivity, b) Seebeck coefficient and c) power factor. 
As observed in Figure 5.3.1 (a), when EMIM:TFSI treated films were manufactured from $\mathrm{v} / \mathrm{v} \%$ solutions $>1.5 \%$, there is a significant increase in electrical conductivity.

The concentration based increment in electrical conductivity is also observed in other PEDOT:PSS ionic liquid composites. ${ }^{369}$ For the pristine PEDOT:PSS film, its electrical conductivity at $40{ }^{\circ} \mathrm{C}$ was measured as $3.4 \mathrm{~S} \mathrm{~cm}^{-1}$ which is in line with literature values. ${ }^{368,370}$ When EMIM:TFSI was added into PEDOT:PSS solutions at $0.5 \%$ and $1 \%$, there were no significant changes to the electrical conductivities of the films.

The 1.5\% EMIM:TFSI film, however, exhibited an increase in electrical conductivity to $451 \mathrm{~S} \mathrm{~cm}^{-1}$. When compared to other ionic liquids in literature such as (BMIM:TFB with $136 \mathrm{~S} \mathrm{~cm}^{-1}$ and BMIM:CL with $49 \mathrm{~S} \mathrm{~cm}^{-1}$ ) at the same concentrations, the value obtained in this study is higher $\left(451 \mathrm{~S} \mathrm{~cm}^{-1}\right)$ is higher. ${ }^{216}$ EMIM:TCB however, yields a slightly higher electrical conductivity at the same concentration $\left(500 \mathrm{~S} \cdot \mathrm{cm}^{-1}\right) .{ }^{369}$

When increasing the concentration of EMIM:TFSI to $2.0 \%$ the electrical conductivity at $40{ }^{\circ} \mathrm{C}$ increased further to $737 \mathrm{~S} \mathrm{~cm}^{-1}$ and further still to $1439 \mathrm{~cm}^{-1}$ for the $2.5 \%$ treatment. Compared to internal solvent treatments such as DMSO (100-600 $\left.\mathrm{S} \mathrm{cm}^{-1}\right),{ }^{179,337,371}$ and ethylene glycol (400-1000 $\left.\mathrm{S} \mathrm{cm}^{-1}\right)^{211,337}$ the 2.5\% EMIM:TFSI is highly far more conductive. EMIM:TCB, in comparison, can yield conductivities of up to $2104 \mathrm{~S} \mathrm{~cm}^{-1}$. 337,369

Whilst EMIM:TFSI treated films in comparison yield a slightly lower electrical conductivity at $40{ }^{\circ} \mathrm{C}$, when the temperature is elevated the electrical conductivity for $\geq 1.5 \%$ EMIM:TFSI films exhibit a lower drop in electrical conductivity. Although the electrical conductivity improves significantly with the addition of EMIM:TFSI, it was not possible to increase the concentration beyond $2.5 \%$ due to the formation of coagulates which impeded spin coating. It is however observed that if the film synthesis conditions are changed then it is possible to make a film with $\geq 13 \%$ EMIM:TFSI treatment. For example, when one wants to make a $\geq 13 \%$ EMIM:TFSI treated film then one would need to introduce the EMIM:TFSI to the film and spin it almost immediately without ultrasonicating it or heating it to aid in mixing. However, this would lead to inhomogeneous films thereby making it hard to control film production. 
There is no clear pattern observed in Figure 5.3.1 (b) showing that EMIM:TFSI treatment significantly affects (improves or reduces) the Seebeck coefficient of the composite films throughout majority of the temperature profile measured. This suggest that EMIM:TFSI does not reduce polythiophene films. Figure 5.3.1 (c) shows that the resultant power factors for the 1.5, 2.0 and 2.5\% EMIM:TFSI films are significantly higher than that of the pristine PEDOT:PSS across the temperature range studied. The PF for the pristine PEDOT:PSS at $40{ }^{\circ} \mathrm{C}$ is $0.04 \mu \mathrm{W} \mathrm{m}^{-1} \mathrm{~K}^{-2}$ and peaks at $0.1 \mu \mathrm{W} \mathrm{m}{ }^{-1}$ $\mathrm{K}^{-2}$ at $177{ }^{\circ} \mathrm{C}$. The power factor for the $2.5 \%$ EMIM:TFSI at $40{ }^{\circ} \mathrm{C}$ however, is much higher $\left(28 \mu \mathrm{W} \mathrm{m} \mathrm{m}^{-1} \mathrm{~K}^{-2}\right)$. This value is comparable to optimized post DMSO and ethylene glycol treatments. ${ }^{337}$ The PF reaches an optimum of $29 \mu \mathrm{W} \mathrm{m}{ }^{-1} \mathrm{~K}^{-2}$ at 109 ${ }^{\circ} \mathrm{C}$. The 1.5 and $2.0 \%$ films show a PF of $6 \mu \mathrm{W} \mathrm{m}{ }^{-1} \mathrm{~K}^{-2}$ and $14 \mu \mathrm{W} \mathrm{m}{ }^{-1} \mathrm{~K}^{-2}$, respectively at $40{ }^{\circ} \mathrm{C}$, whilst the 0.5 and $1 \%$ EMIM:TFSI films exhibit a similar PF to pristine PEDOT:PSS due to their equally low electrical conductivity and their corresponding negligible increases in Seebeck coefficients.

\subsubsection{Post Treatment of EMIM:TFSI Films with $\mathrm{NaBH}_{4}$}

Five PEDOT:PSS/EMIM:TFSI composite films were post treated with a $1 \% \mathrm{w} / \mathrm{v}$ solution of $\mathrm{NaBH}_{4}$ in DMSO for $1 \mathrm{~min}$ at room temperature plus a control treatment of pure DMSO. Figure 5.3.2.1 shows thermoelectric properties of the control films meanwhile Figure 5.3.2.2 represents the thermoelectric properties of the 5 PEDOT:PSS/EMIM:TFSI composites. 


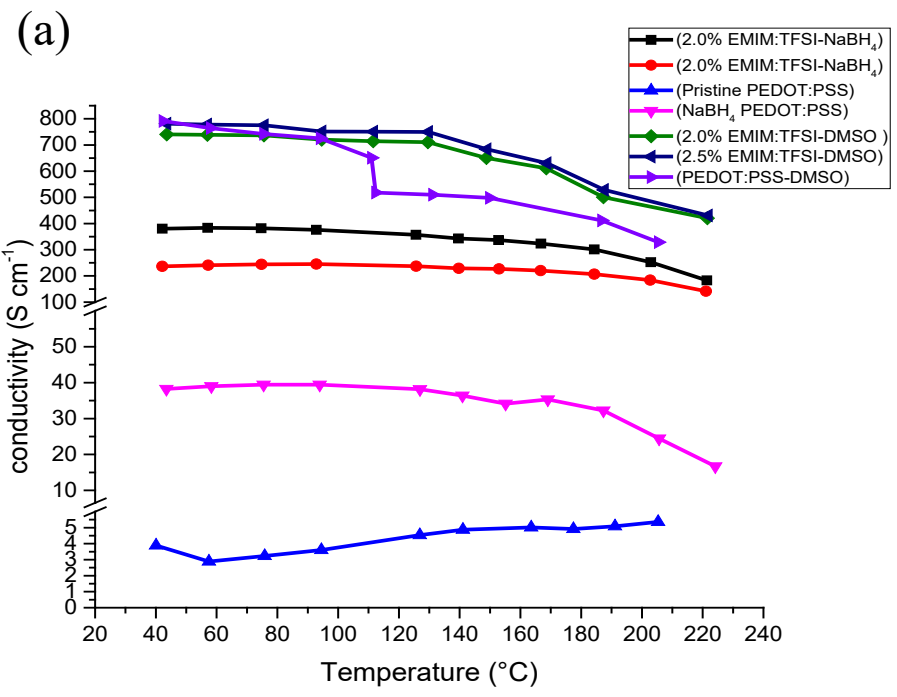

(b)

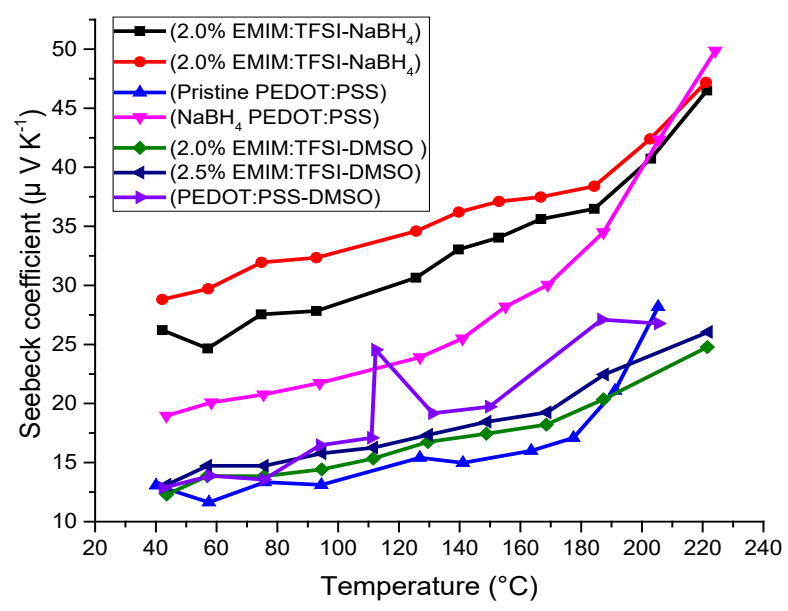

(c)

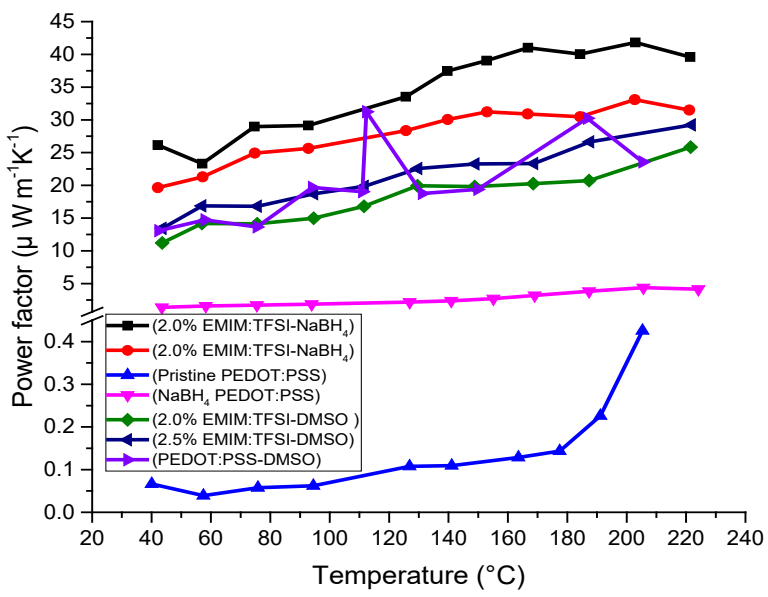


Figure 5.3.2.1 depicting thermoelectric data of EMIM:TFSI, EMIM:TFSI-NABH $\mathrm{H}_{4}$ and DMSO controls.

Because literature shows that DMSO treatments improves electrical conductivity it was necessary to run controls with films treated only with DMSO post treatment to ascertain to what extent it may have been involved in improving the electrical conductivity of the $\mathrm{NaBH}_{4}$ films as well as the Seebeck coefficient. ${ }^{179,209,372}$ The pure DMSO treatment on the films also served as a control to compare if it would further improve the electrical conductivity when treated on the PEDOT/EMIM:TFSI composites. As observed in Figure 4.3.1 (a) the 2 and 2.5\% EMIM:TFSI films have an electrical conductivity of 737 and $1439 \mathrm{~S} \mathrm{~cm}^{-1}$ respectively. When both films are treated with a pure DMSO solution as shown in Figure 5.3.2.1 (a) neither the 2 nor 2.5\% EMIM:TFSI films exhibit an electrical conductivity above $800 \mathrm{~S} \mathrm{~cm}^{-1}$. This shows that further electrical conductivity via DMSO post treatment is not possible on the EMIM:TFSI films. As observed in Figure 5.3.2.1 (b), the presence of DMSO is not responsible for the improved Seebeck coefficient in the films treaded with $\mathrm{NaBH}_{4}$ solution. Films treated with DMSO post treatment but without $\mathrm{NaBH}_{4}$ treatment do not have improved Seebeck coefficient.

Figure 5.3.2.2 (a) shows that $\mathrm{NaBH}_{4}$ treatment reduces the expected electrical conductivity of the EMIM:TFSI composite films. The highest electrical conductivity observed for the EMIM:TFSI-NaBH 4 films was $380 \mathrm{~S} \mathrm{~cm}^{-1}$ at $40{ }^{\circ} \mathrm{C}$ for the $2.0 \%$ films. Although it is expected for the $2.5 \%$ film to have higher electrical conductivity, this can be explained due to the treatment mechanism. When treated with the reducing agent the kinetics may vary slightly due to difficulty control redox of thiophene molecules, hence why the small difference in electrical conductivity between the $2.0 \%$ and $2.5 \%$ film. The $1 \% \mathrm{DMSO}_{\mathrm{NaBH}}$ solution is a strong reducing agent and thereby further studies using a weaker reductant may potentially yield more control regarding the extent of reduction to better optimise the trade-off between loss of electrical conductivity and improved Seebeck coefficient.

Figure 5.3.2.2 (a) depicts the Seebeck coefficient data for the $\mathrm{NaBH}_{4}$ treated films. pristine PEDOT:PSS treated with $\mathrm{NaBH}_{4}$ has a Seebeck coefficient of $20 \mu \mathrm{V} \mathrm{K}{ }^{-1}$, which is similar to values seen in the literature. ${ }^{373}$ This is double that of untreated EMIM: PEDOT:PSS composite films, as shown in this study and elsewhere by 
others. ${ }^{372,374,375}$ It is of note that the electrical conductivity for the $\mathrm{NaBH}_{4}$ treated PEDOT:PSS film (please see Figure 5.2.2.1 (a) is higher than that of the pristine PEDOT:PSS. This is due to DMSO in the $\mathrm{NaBH}_{4}$ solution.

The 1\% EMIM:TFSI, $\mathrm{NaBH}_{4}$ treated film (Figure 5.3.2.2 (b) exhibits the least significant increase in Seebeck coefficient across the temperature range, as it starts at $9.7 \mu \mathrm{V} \mathrm{K}^{-1}$ at $40{ }^{\circ} \mathrm{C}$ and reaches a Seebeck coefficient of $42 \mu \mathrm{V} \mathrm{K}^{-1}$ at $200{ }^{\circ} \mathrm{C}$. This is significantly higher (across the temperature range), however, than the non- $\mathrm{NaBH}_{4}$ treatment sample (Figure 5.3.1 (b). A more pronounced increase in the Seebeck coefficient across the temperature range is observed for the $0.5 \% \mathrm{EMIM}_{\mathrm{TFSI} \mathrm{NaBH}}$ film, which begins at $22 \mu \mathrm{V} \mathrm{K}^{-1}$ at $40{ }^{\circ} \mathrm{C}$ and steadily rises to $56 \mu \mathrm{V} \mathrm{K}^{-1} 220{ }^{\circ} \mathrm{C}$. A yet more pronounced increase in the Seebeck coefficient is seen in the 1.5, 2.0 and 2.5\% EMIM:TFSI NaBH 4 composite films. The highest Seebeck coefficient observed from all the films across the temperature range is attributed to the 2.5\% EMIM:TFSI $\mathrm{NaBH}_{4}$. At $40{ }^{\circ} \mathrm{C}$ the film exhibits a Seebeck coefficient of $30 \mu \mathrm{V} \mathrm{K}^{-1}$ which increases to a maximum of $33 \mu \mathrm{V} \mathrm{K}^{-1}$ above $200{ }^{\circ} \mathrm{C}$. It is of note that the presence of the EMIM:TFSI in $\mathrm{NaBH}_{4}$ treated PEDOT:PSS films, causes a larger increase in the Seebeck coefficient compared to PEDOT:PSS films treated with $\mathrm{NaBH}_{4}$ alone.

The optimal PF (Figure 5.3.2.2 (c) is seen for 2\% EMIM:TFSI-NaBH 4 which is at $40{ }^{\circ} \mathrm{C}$ is $26 \mu \mathrm{W} \mathrm{m}{ }^{-1} \mathrm{~K}^{-2}$ and reaches a maximum of $42 \mu \mathrm{W} \mathrm{m}{ }^{-1} \mathrm{~K}^{-2}$ at $200{ }^{\circ} \mathrm{C}$. The $\mathrm{PF}$ is comparable to the 2.5\% EMIM:TFSI (Figure 4.3.1 (c) at lower temperatures, however above $120^{\circ} \mathrm{C}$ the $\mathrm{PF}$ is significantly higher. This shows the potential to further increase the thermoelectric performance of EMIM:TFSI ionic liquid films with reduction via $\mathrm{NaBH}_{4}$. 


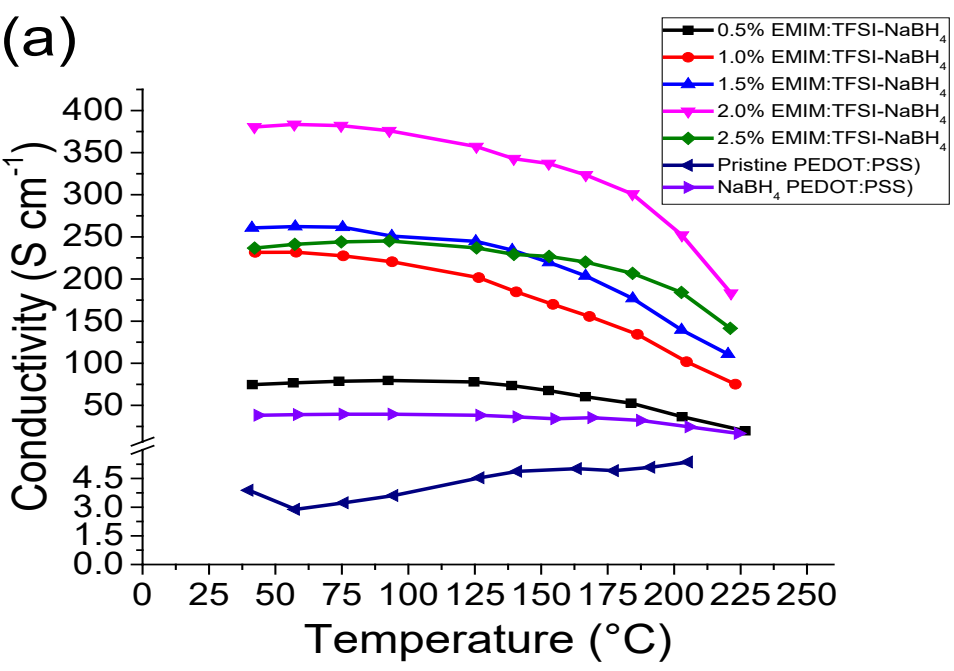

(b)
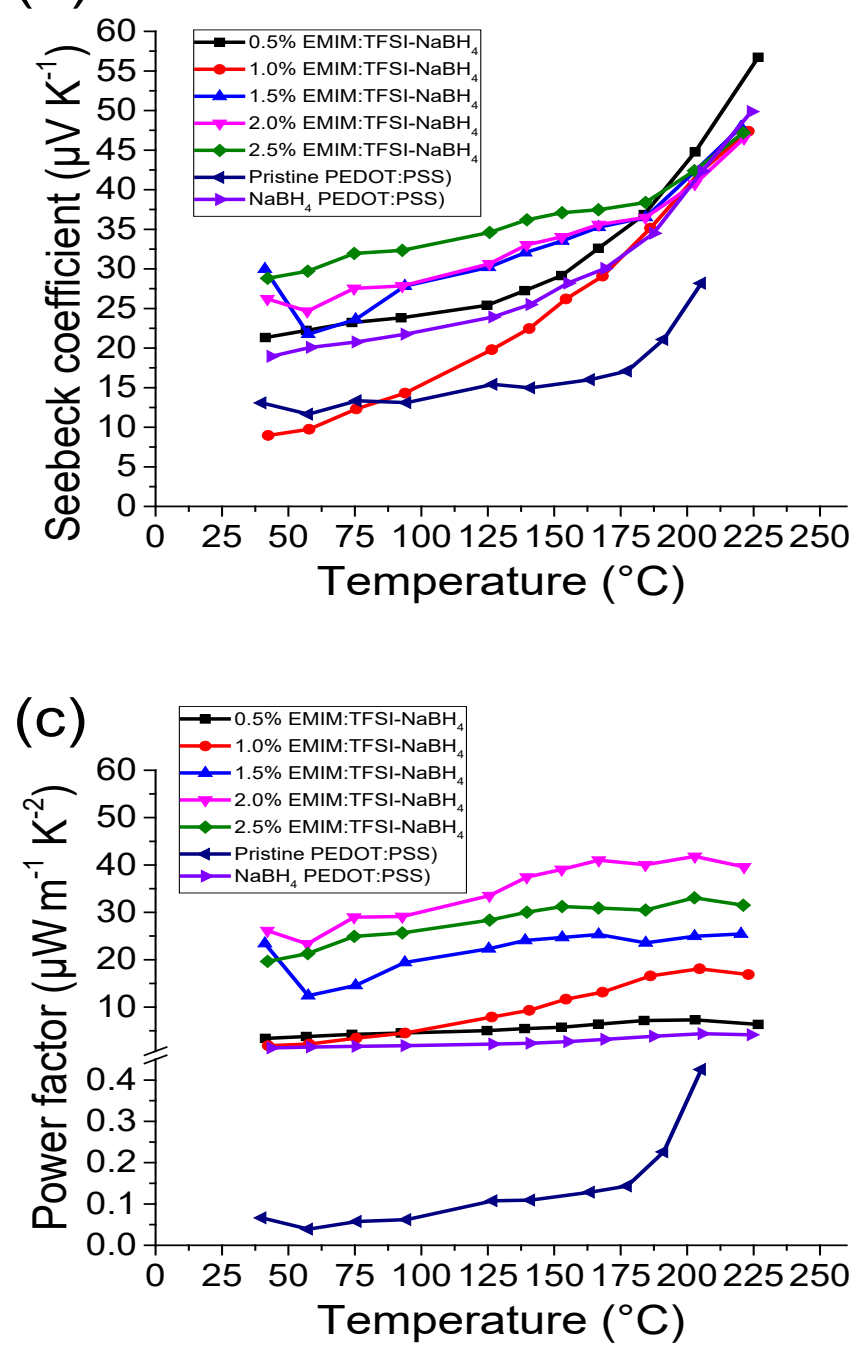

Figure 5.3.2.2 Thermoelectric data for EMIM:TFIS films treated with the 1\% $\mathrm{w} / \mathrm{v}$ $\mathrm{NaBH}_{4}$ solution, (a) denotes the electrical conductivity, (b) depicts the Seebeck coefficient and (c) the power factor. 


\subsubsection{Morphology of PEDOT/EMIM:TFSI composites}

Figure 5.3.3.1 depicts the optical micrograph for all films studied in this chapter.
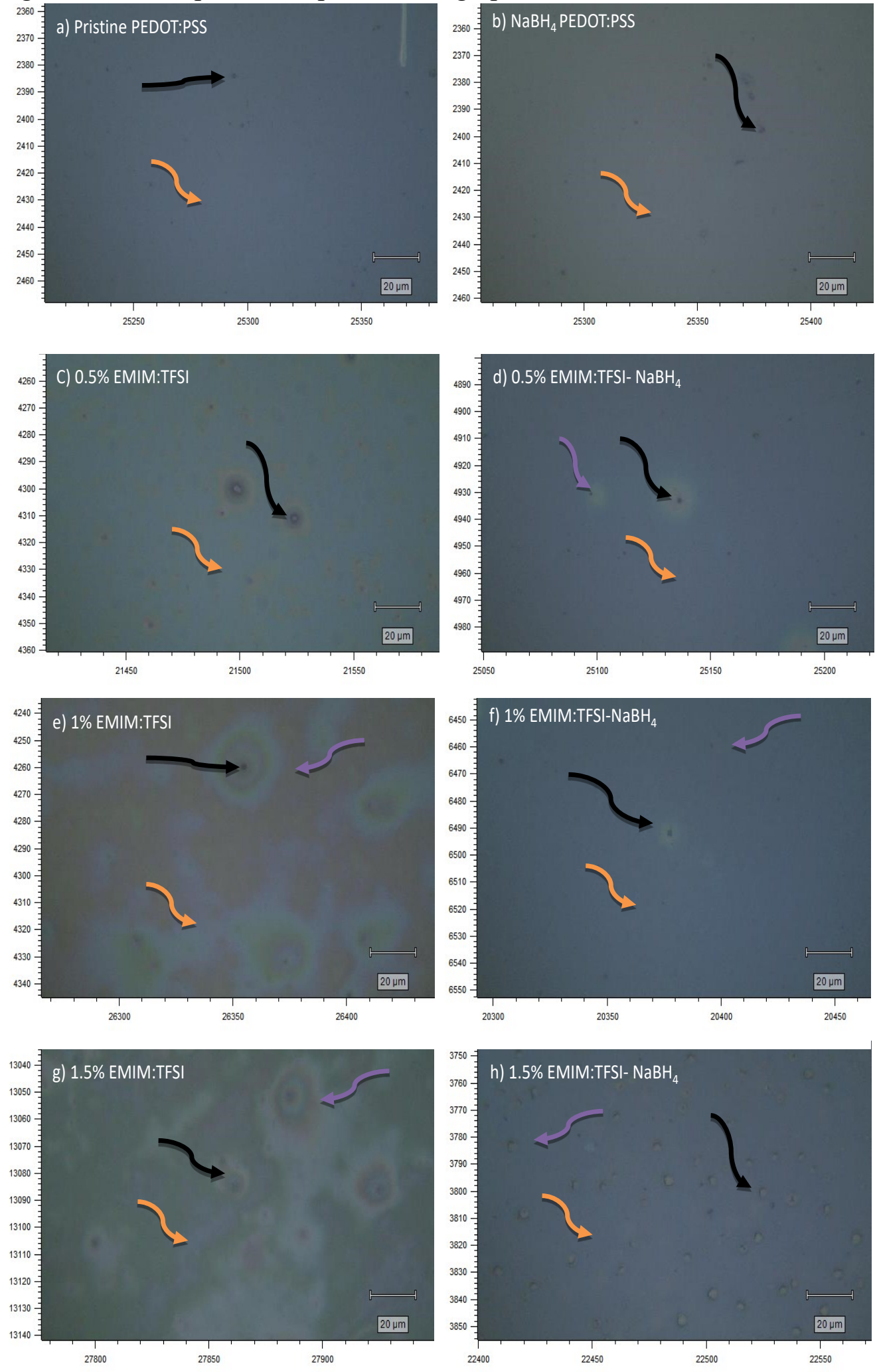

$\mathbf{1 0 6} \mid \mathrm{P}$ a g e 

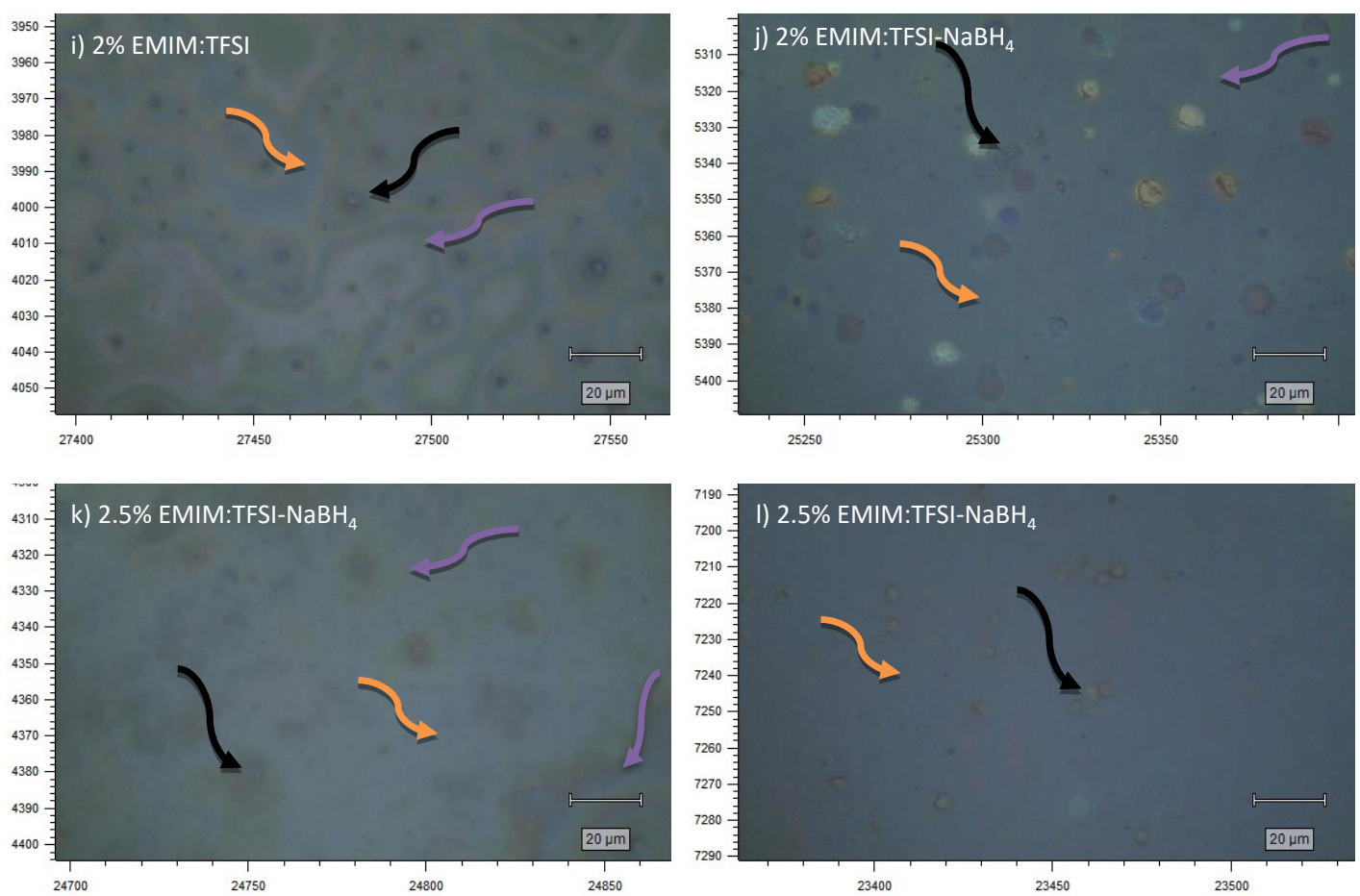

Figure 5.3.3.1 depicting the optical micrographs for the Controls (a), pristine PEDOT:PSS, and (b), the NaBH 4 PEDOT:PSS film. The EMIM:TFSI treated films are represented as (c), (e), (i) and (k). The EMIM:TFSI-NaBH 4 treated films are represented as $(d),(f),(j)$ and $(l)$.

Key

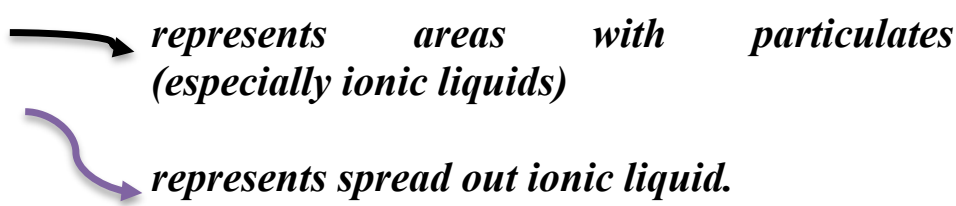

represents area of PEDOT:PSS

When comparing the controls (a) and (b) to (c) the 0.5\% EMIM:TFSI, it is evident there is an emergence of new circular rings. As one observes the remaining EMIM:TFSI treated samples, not only are the rings in all the films but there is also a general increment in the quantity of the rings. The size however of the rings are different ranging from $<5 \mu \mathrm{m}$ to $>15 \mu \mathrm{m}$. As the rings are not present in the controls it is reasonable to deduce that they represent ionic liquid dense areas. These areas are probably brought about by phase separation of the PEDOT:PSS solution and ionic liquid during spin coating via the centrifugal force. 
The presence of identifiable ionic liquid spots indicate that the films have ionic liquid residues. This is not surprising as the boiling point of EMIM:TFSI is around $190{ }^{\circ} \mathrm{C}$ depending on the grade. In reference to the annealing conditions $\left(<120^{\circ} \mathrm{C}\right)$ it makes sense. When comparing the EMIM:TFSI (left hand side) samples to their $\mathrm{NaBH}_{4}$ counterparts (right hand side) of figure, there is a clear qualitative difference. The colour of the film's changes to a bluish hue. It is possible that the difference can be due in part to an ionic liquid absorption or a difference in thickness between the two types of films. This change to a blueish hue however more likely indicates a redox change within the thiophene molecules as was discussed in chapter $4.6 .^{348,376}$ This is supported by other studies that report a deeper blue hue on reduction of PEDOT:PSS films. ${ }^{351,377}$ Still, it appears as though it is not just reduction taking place with the DSMO-NaBH 4 treatment as the area dubbed as (spread out ionic liquid) decreases substantially. For example, when comparing, the 2.5\% EMIM:TFSI to the $2.5 \%$ EMIM:TFSI-NaBH 4 there is a larger area of what is dubbed in the key (represents area of PEDOT:PSS). This indicates that post DMSO-NaBH4 treatment also washes off at least "some" surface ionic liquid however it is argued that a significant amount is removed.

\subsubsection{Mechanisms for the Improved Electrical Conductivity of EMIM:TFSI and EMIM:TFSI $\mathrm{NaBH}_{4}$ Films}

The improved electrical conductivity in the EMIM:TFSI and EMIM:TFSI-NaBH 4 films relative to pristine PEDOT:PSS can be explained by 2 mechanisms. The first mechanism is well established for polar solvents and arises from the conformation change in the PEDOT:PSS back bone from a coiled benzoid structure to a linear/expanded coil quinoid structure as was explored in chapter 4. Figure 5.3.4.1 depicts the different PEDOT conformations. ${ }^{181,298,372,378}$ The interaction among linear PEDOT chains will understandably be stronger than coiled chains. ${ }^{379}$ The stronger interactions have been explained to form better $\pi-\pi$ stacking thereby improving the charge carrier transport channels. ${ }^{181,361}$ These linear chains therefore would lead to more ordered intra chain transport. Raman spectroscopy can be utilized to indicate which structure (benzoid or quinoid) is dominant in the thiophene backbone via the position of the symmetrical stretching of the $\mathrm{C}_{\alpha}=\mathrm{C}_{\beta}$ typically found around $1440 \mathrm{~cm}^{-1}$ 
(please refer to Figure 4.7.3 (C)). ${ }^{300,380} \mathrm{~A}$ red shift which is linked to a benzoid to quinoid shift was observed for all EMIM:TFSI composite films as seen in Figure 5.3.4.2 (a) as well as for the EMIM:TFSI-NaBH 4 composite films as expressed in Figure 5.3.4.2 (b). The EMIM:TFSI-NaBH 4 films presented in Figure 5.3.4.2 (b) exhibit the most significant red shift. This is most likely due to the $\mathrm{NaBH}_{4}$ treatment. Figure 5.3.4.3 depicts the controls necessary to determine if the DMSO is responsible for the further red shift observed or if the significant red shift is due to DMSO-NaBH 4 treatment. Figure 5.3.4.3 shows 2.0\% and 2.5\% EMIM:TFSI-DMSO, films with a less

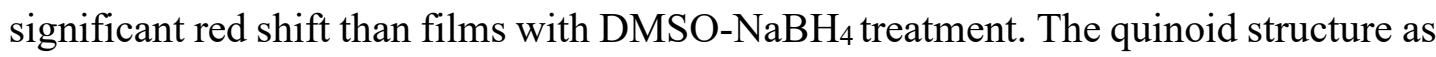
shown in Figure 5.3.4.1 (a) has restricted rotation around the $\mathrm{sp}^{2}$ carbon double bond on the thiophene backbone thereby adopting a planer structure with more crystalline packing relative to the benzoid (Figure 5.3.4.1 (b) structure in which more thiophene units adopt a helical structure with lower molecular ordering that impedes hopping of charge carriers (polarons) within and between the polymer chains. ${ }^{33,34}$

Despite the significant red shift (indicating improved carrier mobility) in $\mathrm{NaBH}_{4}$ films, the conductivity in these films is lower than for the EMIM:TFSI films due to reduced charge carriers (shown later in the UV-Vis-NIR). pristine PEDOT:PSS is included in Figure 5.3.4.2 as a baseline. The peaks at $1365 \mathrm{~cm}^{-1}$ and $1500 \mathrm{~cm}^{-1}$ correspond to the $\mathrm{C}_{\beta}-\mathrm{C}_{\beta}$ inter ring stretching vibration and the asymmetrical $\mathrm{C}_{\alpha}=\mathrm{C}_{\beta}$ vibrations, respectively. ${ }^{300,380}$ PSS bond stretching and bending vibrations can be seen at $1580 \mathrm{~cm}^{-1}$. 


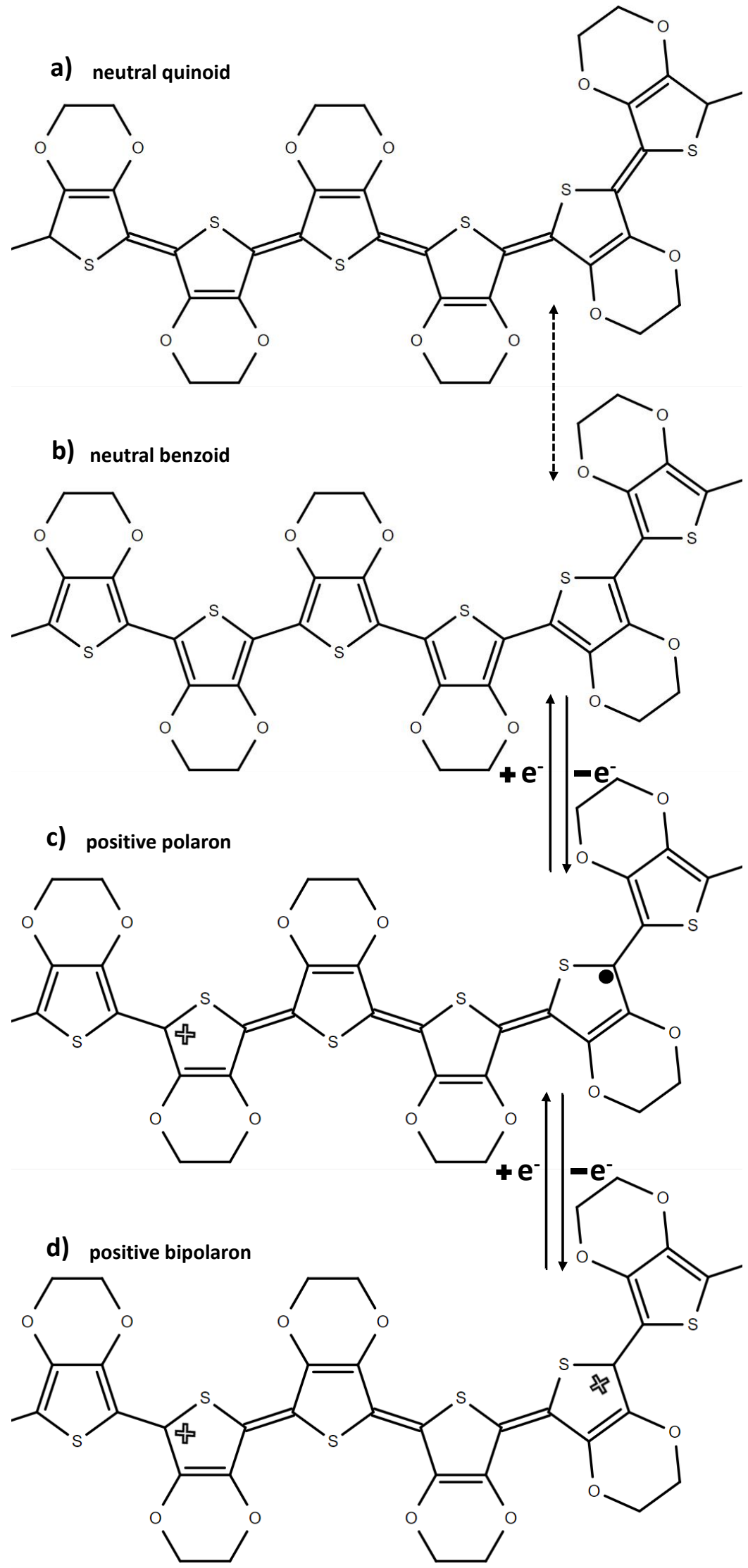

Figure 5.3.4.1. The resonance forms of Poly(3,4-ethylenedioxythiophene) (PEDOT) thiophene back bone, (a) denotes the quinoid form, meanwhile (b) is the benzoid form. (c) and (d) represent the positive polaron and bipolaron phase, respectively. 

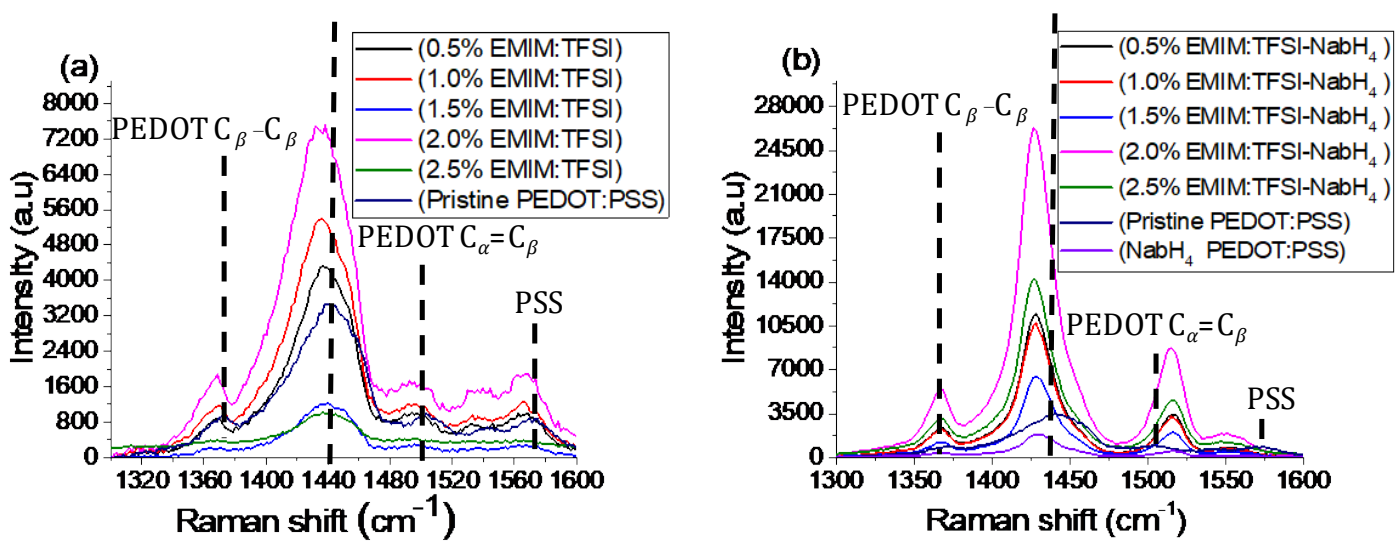

Figure 5.3.4.2 Raman spectroscopy of all films in this study, (a) denotes the EMI:TFSI treated films from $0 \%$ to $2.5 \%$ concentrations, meanwhile (b) corresponds to the $\mathrm{NaBH}_{4}-\mathrm{DMSO}$ solution treatment on the $0 \%$ to $2.5 \%$ EMIM:TFSI films.

The improved electrical conductivity is also in part due to a second mechanism attributed to a selective removal of PSS over PEDOT. Internal treatment with ionic liquids on PEDOT:PSS where PSS is selectively removed over PEDOT leads to PEDOT chain reorientation to a more conductive linear form. ${ }^{216,369,381,382}$ This selective PSS removal is evidenced by XPS quantification shown in Figure 5.3.4.4. The detailed quantification can be seen in Table 5.3.4.1.

The peak intensity of PSS is roughly $12 \%$ more than PEDOT in pristine PEDOT:PSS, which is similar to published results. ${ }^{383,384}$ Please note during quantification the PEDOT section has an asymmetric tail towards the higher binding energy depicting the polaron and bipolaron thiophene molecules therefore in reality the concentration of PEDOT after peak integration is higher than the visual intensity. In the $2.5 \%$ EMIM:TFSI-NaBH 4 treatment the relative intensity ratio between PEDOT and PSS is seen to be significantly increased in favour of PEDOT.

Figure 5.3.4.5 depicts the high-resolution Sulphur 2p (S 2p) spectra for the 2.5\% EMIM:TFSI (a), pristine PEDOT:PSS, (b), and the 2.5\% EMIM:TFSI-NaBH 4 (d) film with peak fitting in a range consistent with literature values. ${ }^{309,385,386387}$ Due to the sulphite sulphur in PSS being bonded to highly electronegative oxygen atoms, its peak is at a higher binding energy than the thiophene sulphur in PEDOT which is bonded 
to the less electronegative $\mathrm{sp}^{2}$ hybridized carbons. ${ }^{388,389}$ TFSI is bonded to carbon with 3 electronegative fluorides attached which leads to the sulfonyl sulphur being at a higher still binding energy, as seen in Figure 5.3.4.5 (a) and literature. ${ }^{390}$

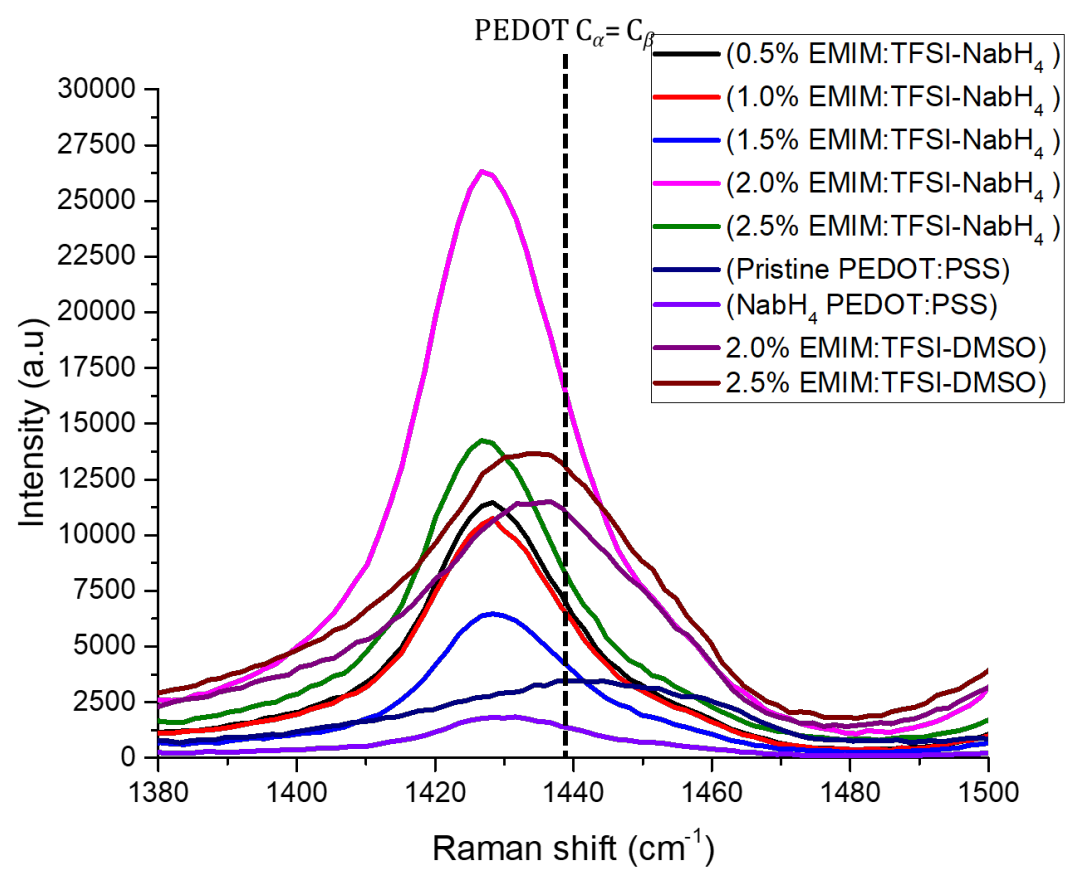

Figure 5.3.4.3 Raman spectra depicting ionic liquid films treated with DMSO, post treatment without $\mathrm{NaBH} 4$ to show that $\mathrm{NaBH} 4$ presence is the major cause for the increased red shift. 


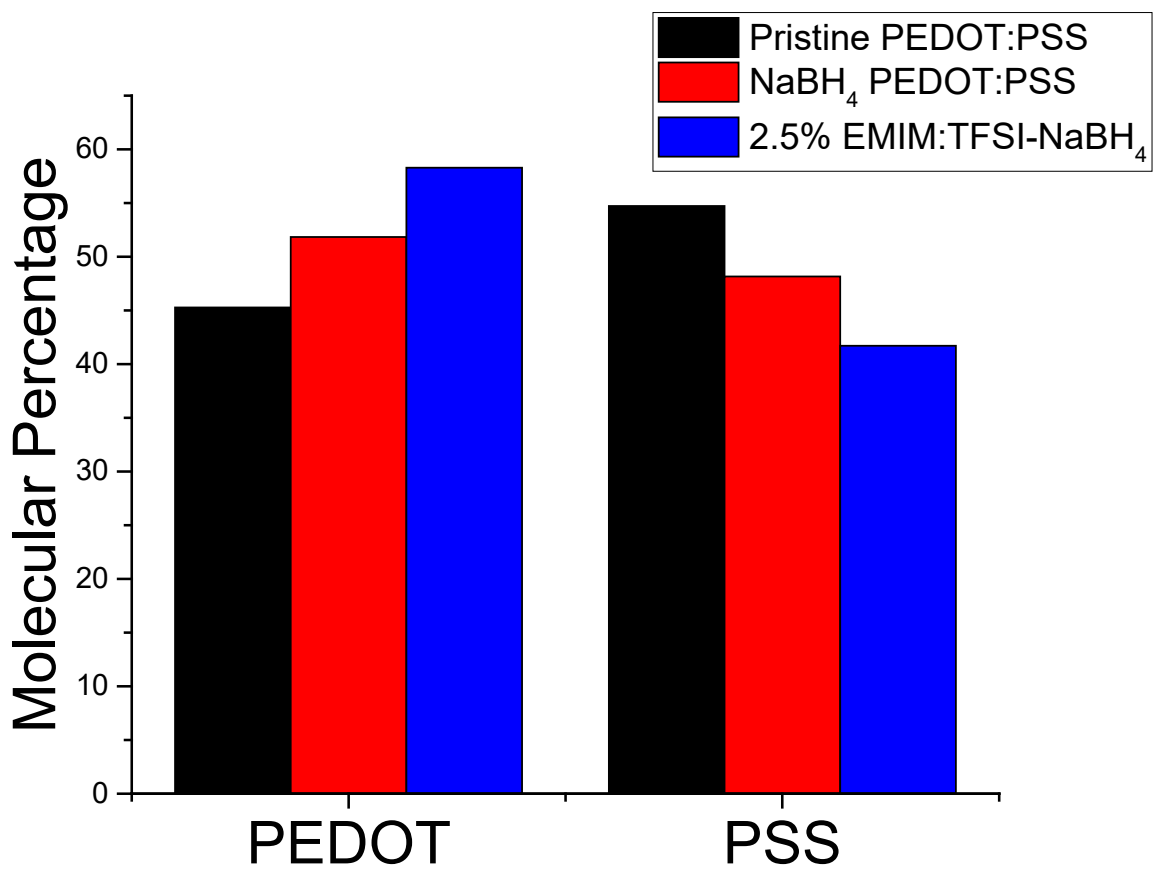

Figure 5.3.4.4 Quantified molecular percentage ratio between PEDOT and poly(4styrenesulfonate) (PSS) in relation to the pristine PEDOT:PSS, the $\mathrm{NaBH}_{4}$

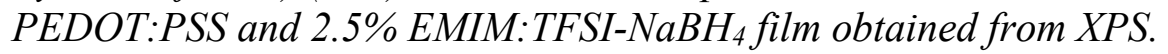



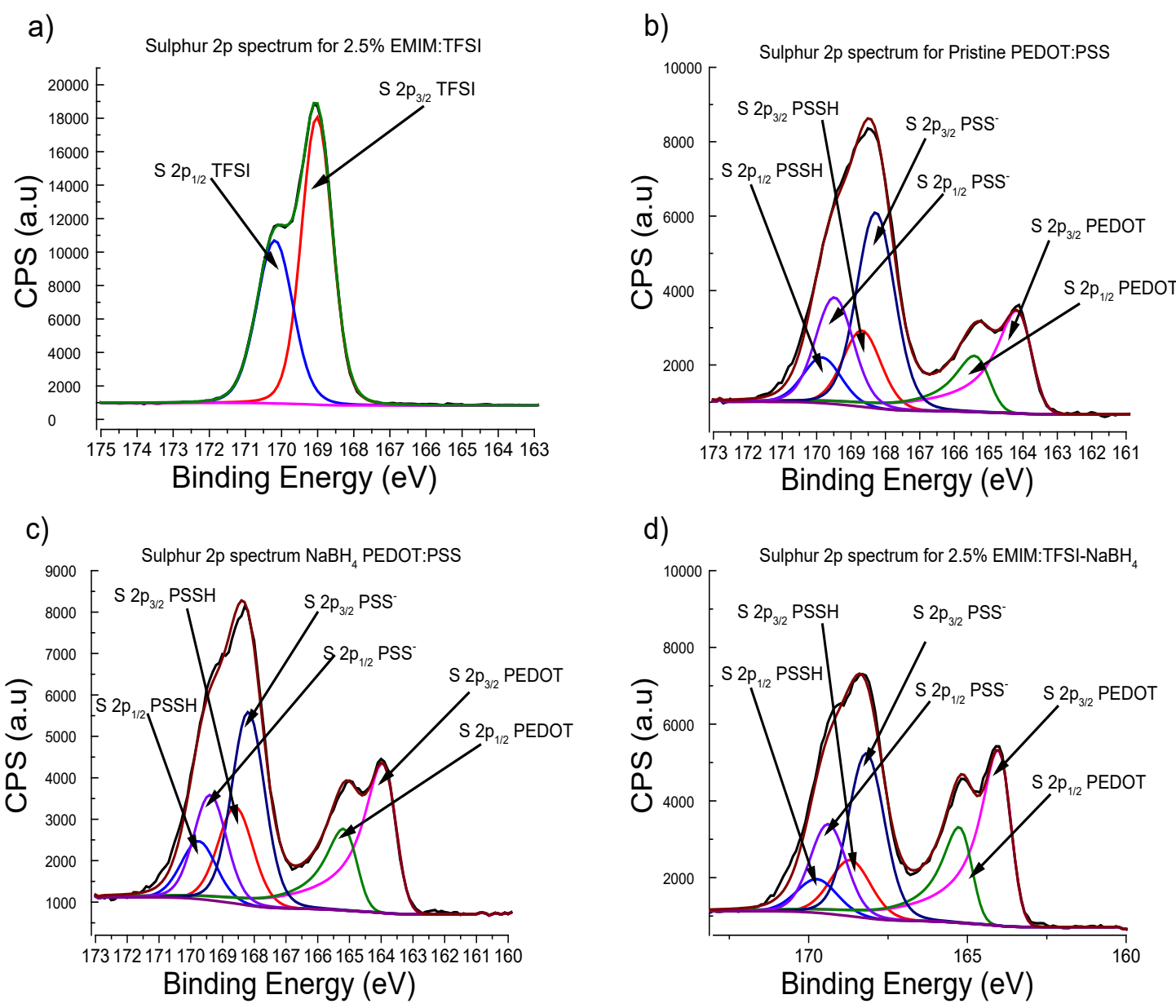

Figure 5.3.4.5 depicting S 2p spectra for the (2\% EMIM:TFSI film (a), whereby there are no signals for PEDOT or PSS thereby indicating the surface of the film is covered in ionic liquid. (b) Depicts the spectrum for pristine PEDOT:PSS whereby PEDOT and PSS regions are labelled appropriately. (c) Depicts the $\mathrm{NaBH}_{4}$ PEDOT:PSS film whereby some increased PEDOT intensity is observed relative to PSS due to removal of PSS via DMSO. (d) Corresponds to the proxy used for the $2.5 \%$ EMIM:TFSI film whereby it is shown that after being washed with reducing agent treatment in DMSO the TFSI is washed off the surface of the film. It is also shown that it is PEDOT concentration is even higher than for the $\mathrm{NaBH}_{4}$ PEDOT:PSS film thereby indicating EMIM:TFSI treatment has significant effect at selectively removing PSS. 
Table 5.3.4.1 depicting the quantification data for the 2.5\% EMIM:TFSI, pristine PEDOT:PSS, 2.5\% EMIM:TFSI NaBH4, and the $\mathrm{NaBH}_{4}$ PEDOT:PSS film with each films corresponding releative peak positions.

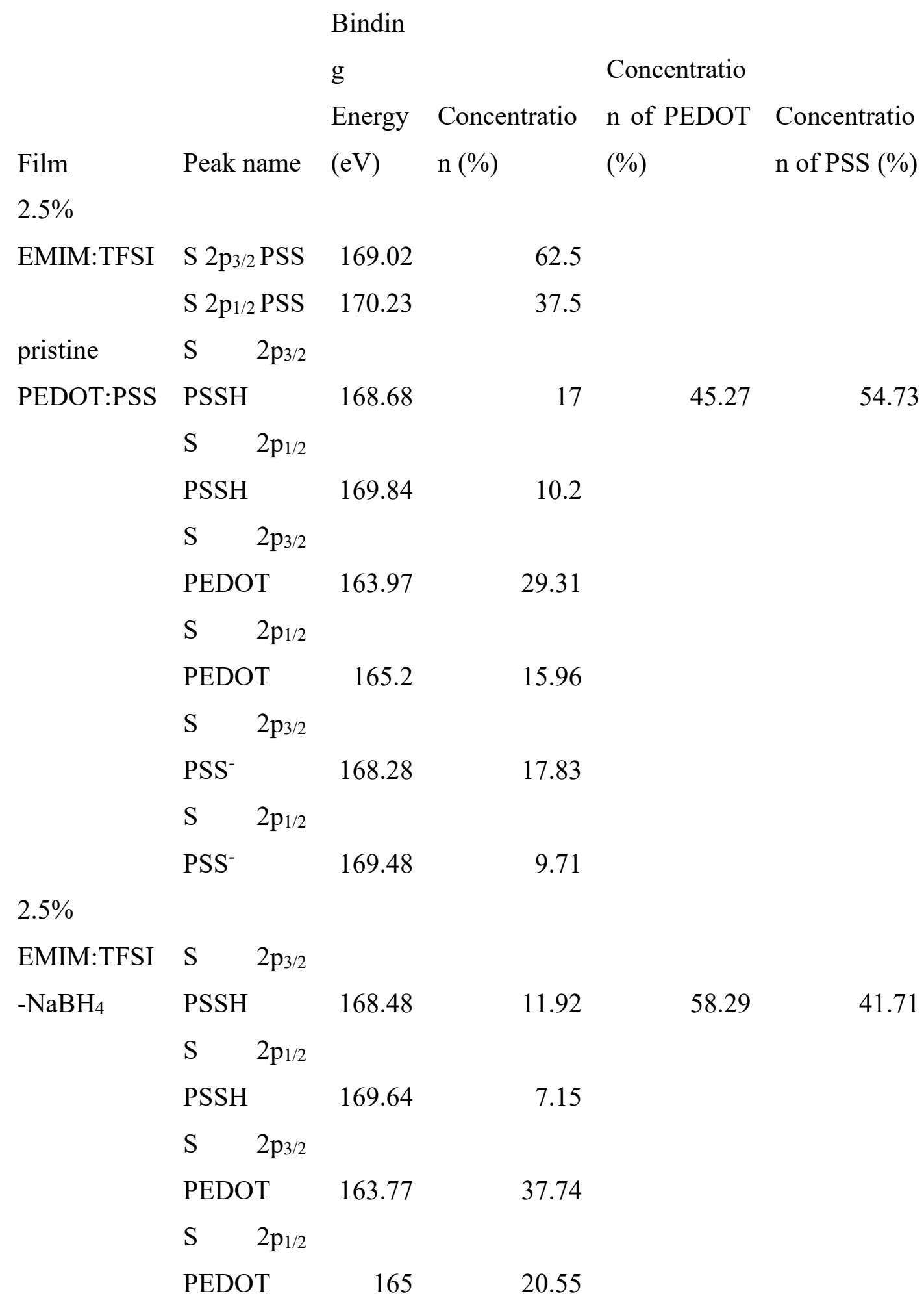




\begin{tabular}{|c|c|c|c|c|c|c|}
\hline & S & $2 p_{3 / 2}$ & & & & \\
\hline & $\mathrm{PSS}^{-}$ & & 168.08 & 14.66 & & \\
\hline & $\mathrm{S}$ & $2 \mathrm{p}_{1 / 2}$ & & & & \\
\hline & $\mathrm{PSS}^{-}$ & & 169.28 & 3.37 & & \\
\hline $\mathrm{NaBH}_{4}$ & S & $2 \mathrm{p}_{3 / 2}$ & & & & \\
\hline PEDOT:PSS & PSSH & & 168.59 & 17.3 & 51.83 & 48.17 \\
\hline & S & $2 \mathrm{p}_{1 / 2}$ & & & & \\
\hline & PSSH & & 169.75 & 10.38 & & \\
\hline & S & $2 \mathrm{p}_{3 / 2}$ & & & & \\
\hline & $\mathrm{PEDO}^{-}$ & & 163.77 & 33.56 & & \\
\hline & $\mathrm{S}$ & $2 \mathrm{p}_{1 / 2}$ & & & & \\
\hline & PEDO' & & 165.00 & 18.27 & & \\
\hline & S & $2 \mathrm{p}_{3 / 2}$ & & & & \\
\hline & $\mathrm{PSS}^{-}$ & & 168.19 & 13.27 & & \\
\hline & S & $2 \mathrm{p}_{1 / 2}$ & & & & \\
\hline & $\mathrm{PSS}^{-}$ & & 169.39 & 7.23 & & \\
\hline
\end{tabular}

As shown in Table 5.3.4.1 and Figure 5.3.4.5 (a), the 2.5\% EMIM:TFSI film shows no PEDOT or PSS peak. The peaks at binding energies of 169.02 and $170.23 \mathrm{eV}$ correspond to the TFSI sulphur spin-orbital coupling ( $\left.\mathrm{S} 2 \mathrm{p}_{3 / 2}\right)$ and ( $\left.\mathrm{S} 2 \mathrm{p}_{1 / 2}\right)$ respectively. ${ }^{391}$ XPS has a penetration depth of approximately $10 \mathrm{~nm}$, which shows at least the surface of the $60 \mathrm{~nm}$ EMIM:TFSI composite films were covered with the TFSI anion. Therefore, to determine the effects of EMIM:TFSI treatment on the PEDOT to PSS ratio, the quantified data for the $2.5 \%$ EMIM:TFSI-NaBH 4 can be used as a proxy for the $2.5 \%$ EMIM:TFSI film because they have the same chemical composition with the exception of that the TFSI anion has been washed away from the surface by the $\mathrm{NaBH}_{4}$ treatment thereby leading to PEDOT and PSS peaks being observed and disappearing of TFSI peaks.

To determine that the effects on the PEDOT to PSS ratio are not from DMSO which is present in the $\mathrm{NaBH}_{4}$ solution, a $\mathrm{NaBH}_{4}$ treated pristine film is shown in Figure 5.3.4.5 (c). This is because DMSO is known to induce selective removal of PSS in literature. ${ }^{22,392}$ EMIM:TFSI-NaBH 4 films are shown to have a higher proportion of 
PEDOT than solely $\mathrm{NaBH}_{4}$ treated films, therefore it can be concluded that EMIM:TFSI selectively removes PSS over PEDOT.

\subsubsection{Mechanisms for the Improved Seebeck Coefficient of EMIM:TFSI NaBH 4 Films}

An increase in the Seebeck coefficient of PEDOT:PSS can often be attributed to a reduction of carrier concentration. ${ }^{128,209,393}$ Treatment with $\mathrm{NaBH}_{4}$ results in a redox reaction with PEDOT:PSS as expressed in the equation: ${ }^{394}$

$$
2 \mathrm{NaBH}_{4}+\mathrm{PEDOT}^{2+}+2 \mathrm{PSS}^{-} \rightarrow 2 \mathrm{NaPSS}+\mathrm{PEDOT}+2 \mathrm{BH}_{3}+\mathrm{H}_{2}
$$

As evidenced by Figure 5.3.4.2 the reduced carrier concentration via $\mathrm{NaBH}_{4}$ treatment (Figure 5.3.4.2 (b) can be observed by a reduction of the width and increase in the intensity corresponding to the symmetric and asymmetric $\mathrm{C}_{\alpha}=\mathrm{C}_{\beta}$ stretching of the Raman spectra relative to the films not treated with $\mathrm{NaBH}_{4}$ (Figure 5.3.4.2 (a). This however does not increase the intensity of the PSS region thereby inferring less significant change in the chemical environment. ${ }^{395}$

The redox reaction can result in the change of the resonance form of the PEDOT which can be evidenced by a change in the UV-Vis-NIR absorption spectrum. Figure. 5.3.5.1 shows the UV-Vis-NIR spectra of a $\mathrm{NaBH}_{4}$ treated film in relation to pristine PEDOT:PSS. The $\mathrm{NaBH}_{4}$ film shows an emergence of a polaron peak at $900 \mathrm{~nm}$ (see also Figure 5.3.4.1) and a reduction in the peak extending into the NIR compared to pristine PEDOT:PSS, indicating a reduction in the quantity of bipolarons (see also Figure 5.3.4.1 (d) and an increase of the quantity of polarons in the sample. ${ }^{128,139,209,396}$ This explains the increase of Seebeck coefficient seen in Figure 5.3.2.2 (b) for $\mathrm{NaBH}_{4}$ treated PEDOT:PSS films. A study utilizing EMIM:DCA discovered that DCA had a reducing effect thereby increasing the Seebeck coefficient by reducing the charge carrier concentration. ${ }^{197}$ Figure 5.3.1 reveals however, that the Seebeck coefficient is not significantly affected by EMIM:TFSI treatment. This could be due to the TFSI anion being unstable after donating an electron. This is consistent with the UV-VisNIR spectra as depicted in Figure 5.3.5.1, which shows that the 2.5\% EMIM:TFSI film has no emergence of a polaron peak at $900 \mathrm{~nm}$ or a reduction of the bipolaron region in the NIR. 2.5\% EMIM:TFSI-NaBH 4 film, however, shows a more distinct peak in 
the polaron region and a more reduced bipolaron section compared to the $\mathrm{NaBH}_{4}$ treated pristine PEDOT:PSS films, indicating a stronger reduction in those films. This observation is supported by the larger Seebeck coefficients observed in $\mathrm{NaBH}_{4}$ treated EMIM:TFSI films, as seen in Figure 5.3.2.2 (b).

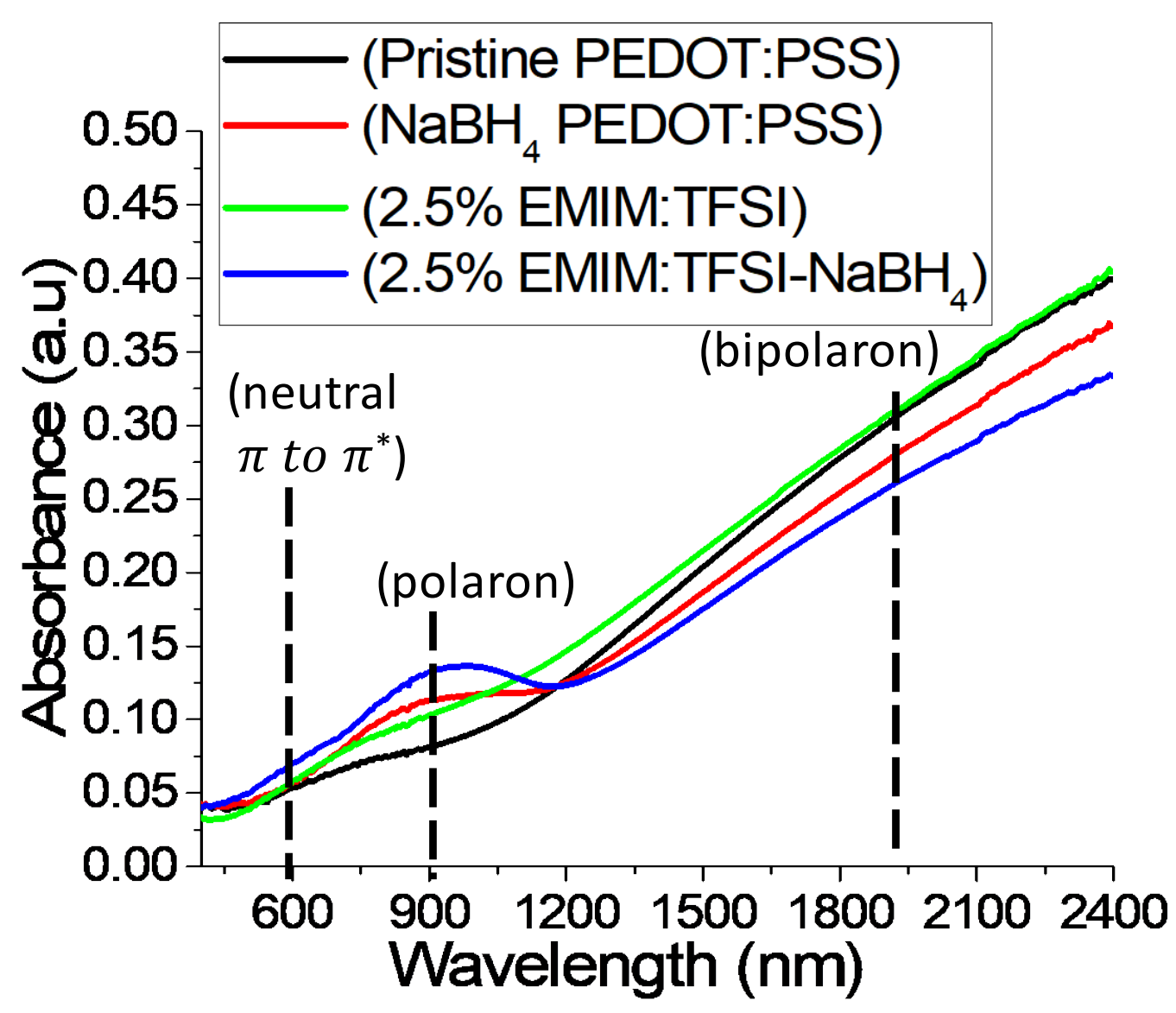

Figure 5.3.5.1 UV-VIS depicting absorbance pristine PEDOT:PSS, NaBH4 PEDOT:PSS, 2.5\% EMIM:TFSI and 2.5\% EMIM:TFSI-NaBH films. $^{2}$

To understand why the EMIM:TFSI-NaBH 4 films have higher Seebeck coefficient than the $\mathrm{NaBH}_{4}$ PEDOT:PSS, it's important to note that whilst the ionic liquid has been confirmed to not affect the Seebeck coefficient, the ionic liquid treatment causes phase separation and the subsequent removal of PSS from the polymer. Under the same conditions when post treated with $\mathrm{NaBH}_{4}-\mathrm{DMSO}$ solution, the reducing agent is in the presence of a higher concentration of PEDOT chains thereby making reduction more effective, due to lower PSS concentration. This consequently leads to a higher Seebeck coefficient.

Figure 5.3.5.2 depicts the overlaid XPS spectra for pristine PEDOT:PSS, 2.5\% EMIM:TFSI $\mathrm{NaBH}_{4}$ and $\mathrm{NaBH}_{4}$ PEDOT:PSS. The XPS data shows a chemical shift 
in the PEDOT region for the $\mathrm{NaBH}_{4}$ treated films to a lower binding energy showing reduction of some thiophene units from bipolarons to polarons has occurred, which agrees with the UV-VIS-NIR observations. Figure 5.3.5.2 in conjunction with Table 5.3.4.1 and Figure 5.3.4.5 show the PEDOT spin-orbital sulphur coupling for the pristine PEDOT:PSS (S 2p $\mathrm{p}_{3 / 2}$ ) shifts from a binding energy of $163.97 \mathrm{eV}$ to $163.77 \mathrm{eV}$ when treated with $\mathrm{NaBH}_{4}$. The 2.5\% EMIM:TFSI-NaBH 4 film shows the same shift from $163.97 \mathrm{eV}$ for pristine PEDOT:PSS (S 2 $\left.\mathrm{p}_{3 / 2}\right)$ to $163.77 \mathrm{eV}$. A similar shift can be observed for the $\left(\mathrm{S}_{2} \mathrm{p}_{1 / 2}\right)$ peaks. This shift is also observed in literature for reducing agent treatment on PEDOT:PSS. ${ }^{209,373}$

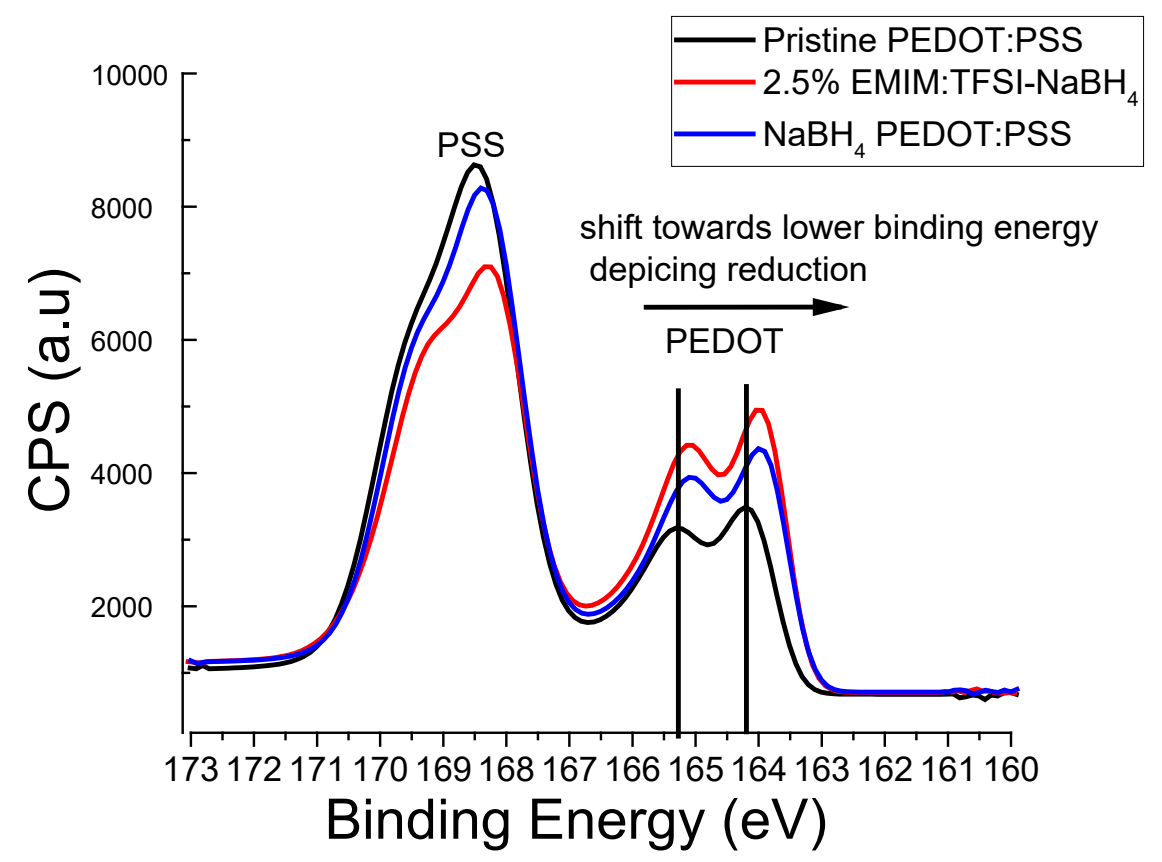

Figure 5.3.5.2 Overlaid XPS spectra showing a lower binding energy shift for $\mathrm{NaBH}_{4}$ treated films relative to pristine PEDOT:PSS indicating reduction.

\subsection{Conclusions}

A thin film PEDOT: PSS ionic liquid composite utilizing the ionic liquid EMIM:TFSI was synthesized with superior thermoelectric properties to pristine PEDOT:PSS. The ionic liquid significantly improved the electrical conductivity from $3 \mathrm{~S} \mathrm{~cm}^{-1}$ to 1439 $\mathrm{S} \mathrm{cm}^{-1}$ for the $2.5 \%$ EMIM:TFSI film thereby leading to a PF of $28 \mu \mathrm{W} \mathrm{m}{ }^{-1} \mathrm{~K}^{-2}$ at 40 ${ }^{\circ} \mathrm{C}$. A further post treatment with $\mathrm{NaBH}_{4}$-DMSO solution was used to improve the Seebeck coefficient which lead to an increase in the PF for most of the films treated 
with this solution relative to the ones treated with only the ionic liquid. At above 140 ${ }^{\circ} \mathrm{C}$ the $2.0 \%$ EMIM:TFSI $\mathrm{NaBH}_{4}$ film was optimized to a $\mathrm{PF}$ of $40 \mu \mathrm{W} \mathrm{m}{ }^{-1} \mathrm{~K}^{-2}$, meanwhile the $2.5 \%$ EMIM:TFSI $\mathrm{NaBH}_{4}$ was optimized to $33 \mu \mathrm{W} \mathrm{m}^{-1} \mathrm{~K}^{-2}$ at the same temperature.

This simple two-step process is fully solution processable and shows the potential for further optimization of the PF of PEDOT:PSS ionic liquid composites by fine tuning the concentration of the $\mathrm{NaBH}_{4}-\mathrm{DMSO}$ solution and finding more precise ways to control the redox of PEDOT films to minimize the depletion of electrical conductivity. Raman showed that there was a conformational change from benzoid to quinoid. XPS confirmed a second mechanism for improved electrical conductivity via a phase separation and the selective removal of PSS. The improved Seebeck coefficient was confirmed to be due to the post treatment with $\mathrm{NaBH}_{4}$-DMSO solution, however, it was discovered that the ionic liquid treated films had higher Seebeck coefficient. The study proved that this discrepancy was due to the ionic liquid removing PSS and exposing the $\mathrm{NaBH}_{4}$-DMSO solution to more PEDOT chains, hence causing further reduction. The UV-VIS and XPS provided evidence for a reduction in charge carriers from bipolarons to polarons with the $\mathrm{NaBH}_{4}-\mathrm{DMSO}$ solution treatment. Changing the synthesis method whereby the coagulation of the solution of PEDOT:PSS ionic liquid does not occur until a higher ionic liquid concentration may prove helpful in optimizing the electrical conductivity of these films, as the results showed a positive correlation between concentration of ionic liquid and electrical conductivity. 
$121 \mid \mathrm{P}$ a g e 


\section{Chapter 6 Synthesis of N-type SWNT- PEDOT:PSS composites}

\subsection{Abstract}

An extremely fast and efficient synthetic route to a novel n-type SWNT-PEDOT:PSS composite was realized through solvothermal treatment with the reducing agent, tetrabutyle ammonium hydroxide (TBAOH) at $120{ }^{\circ} \mathrm{C}$ for 40 seconds. It was discovered by Raman spectroscopy, that films exhibiting n-type characteristics had a corresponding red shift at the $\mathrm{G}^{+}$band. This infers n-type characteristics via electron injection into the carbon nanotubes. It was also discovered that only films that underwent reduction of thiophenes became n-type via analysis of control films without the TBAOH treatment.

\subsection{Introduction}

Due to the difficulty in obtaining stable n-type PEDOT:PSS semiconductors the research lags in comparison to the p-type semiconductor. ${ }^{141}$ In research due to issues pertaining to air stability it's also hard to develop stable n-type organic thermoelectrics with high power factor. Recent studies however have found several synthetic routes where carbon nanotubes can be imbedded within the PEDOT:PSS matrix. ${ }^{234}$ Following a subsequent treatment with certain electron donor molecules such as organic, inorganic bases, or primary amines, n-type characteristics can be observed and deduced through the sign change from the Seebeck coefficient from positive (denoting p-type) to negative (denoting n-type). It has been shown in literature that compositing SWNT into PEDOT:PSS polymer matrix that the thermal conductivity largely remains small, thereby making the SWNT-PEDOT:PSS composites suitable candidates for thermoelectric applications. 


\subsection{Objective and overview}

In this study a concentration of between 5 and $80 \% \mathrm{v} / \mathrm{v}$ SWNT to PEDOT:PSS is explored with the aim of synthesizing a novel thin film n-type SWNT-PEDOT:PSS composite. The origin of the $n$ type Seebeck coefficient is understood through XPS and Raman studies.

\subsection{Materials and methods}

The generalized materials synthesis is described in chapter 3.1 and 3.2.1. A more specific method is expressed in this chapter for the novel n-type SWNTPEDOT:PSS composites. There are three sets of materials used in this study. The first set of films are six films denoting some fundamental controls to derive origins of $n$ type characteristics. The second set correspond to the concentration dependent n-type films denoted as SWNT-PEDOT:PSS-conc and have been treated with TBAOH. The third is the control of p-type films denoted SWNT-PEDOT:PSS-cont, these are made in the same way as the SWNT-PEDOT:PSS-conc films but without the TBAOH treatment.

\subsubsection{Synthesis of the fundamental controls}

a) Six films denoted with a number from 1-6 are utilized as initial controls. The 1-pristine PEDOT:PSS film is composed of a pristine PEDOT:PSS film synthesized as shown in (chapter 3.2.1).

b) The 2-PEDOT:PSS-DMSO film is composed of $5 \% \mathrm{v} / \mathrm{v}$ DMSO treatment on pristine PEDOT:PSS solution and spun as shown in chapter 3.2.1.

c) The 3-pristine PEDOT:PSS-DMSO-TBAOH film is composed of a 5\% v/v DMSO treatment as well as a corresponding TBAOH treatment. After a 2-PEDOT:PSS-DMSO is spun it is then subjected to a post treatment with $\mathrm{TBAOH}$ for 40 seconds at $120{ }^{\circ} \mathrm{C}$ and subsequently washed with isopropyl alcohol 3 times (to quench reduction) and dried in nitrogen stream for 1 minute. 
d) The $4-100 \%$ SWNT-DMSO film is composed of a $5 \%$ DMSO $v / v$ to the SWNT solution (Please refer to chapter 3.1.3) spin coated as explained in chapter 3.2.1.

e) The 5-100\% SWNT-DMSO-TBAOH film is composed of the same way as the $4-100 \%$ SWNT-DMSO with except for an additional TBAOH post treatment as expressed for the 3-pristine PEDOT:PSSDMSO-TBAOH film.

f) The $6-100 \%$ SWNT film is a composed of a pristine SWNT solution spun (please refer to chapter 3.2.1).

\subsubsection{Synthesis of n-type SWNT-PEDOT:PSS-conc films.}

g) Films are denoted as (SWNT-PEDOT:PSS-conc), due to having varying concentration of SWNTs present in each film. Please refer to Figure 6.4.3.1 for pictographic representation of the synthesis.

h) $2000 \mu \mathrm{L}$ of PEDOT:PSS and $100 \mu \mathrm{L}$ of DMSO (5\% v/v) solutions were placed in each of $5(7 \mu \mathrm{L})$ clear glass vials. The vials were subsequently mixed with a specified amount of SWNT solution and labelled with respect to the concentration (v/v) of SWNT $(5,10,20,40$, and $80 \%)$. For example, $200 \mu \mathrm{L}$ of the SWNT solution was mixed with $2000 \mu \mathrm{L}$ of PEDOT:PSS to make the $10 \%$ SWNT composite. This process was repeated for the remaining 4 vials and mixed for 5 minutes using mechanical mixer (vortex).

i) The solutions were then spin coated and annealed (please refer to chapter 3.2.1)

j) After annealing the 5 films were maintained on the hot plate at $120^{\circ} \mathrm{C}$. Subsequently $200 \mu \mathrm{L}$ of TBAOH was pipeted onto each film for 40 seconds.

k) The films were washed with isopropyl alcohol 3 times and then gently dried under nitrogen stream for 1 minute.

1) There was only slight variation in film thickness with each film ranging between 50 and $60 \mathrm{~nm}$ as determined by Dektak surface profilometry. 


\subsubsection{Control for origin of n-type, films dubbed (SWNT- PEDOT:PSS-cont)}

a) The methods from chapter 6.4.2 (h, i, and $\mathrm{k}$ and $\mathrm{l})$ were repeated. The films were maintained on a hot plate for 40 seconds at $120{ }^{\circ} \mathrm{C}$ and a subsequent washing with isopropyl alcohol was conducted as well as drying in a nitrogen stream for 1 minute for consistency. The films, however, were not post treated with $\mathrm{TBAOH}$.

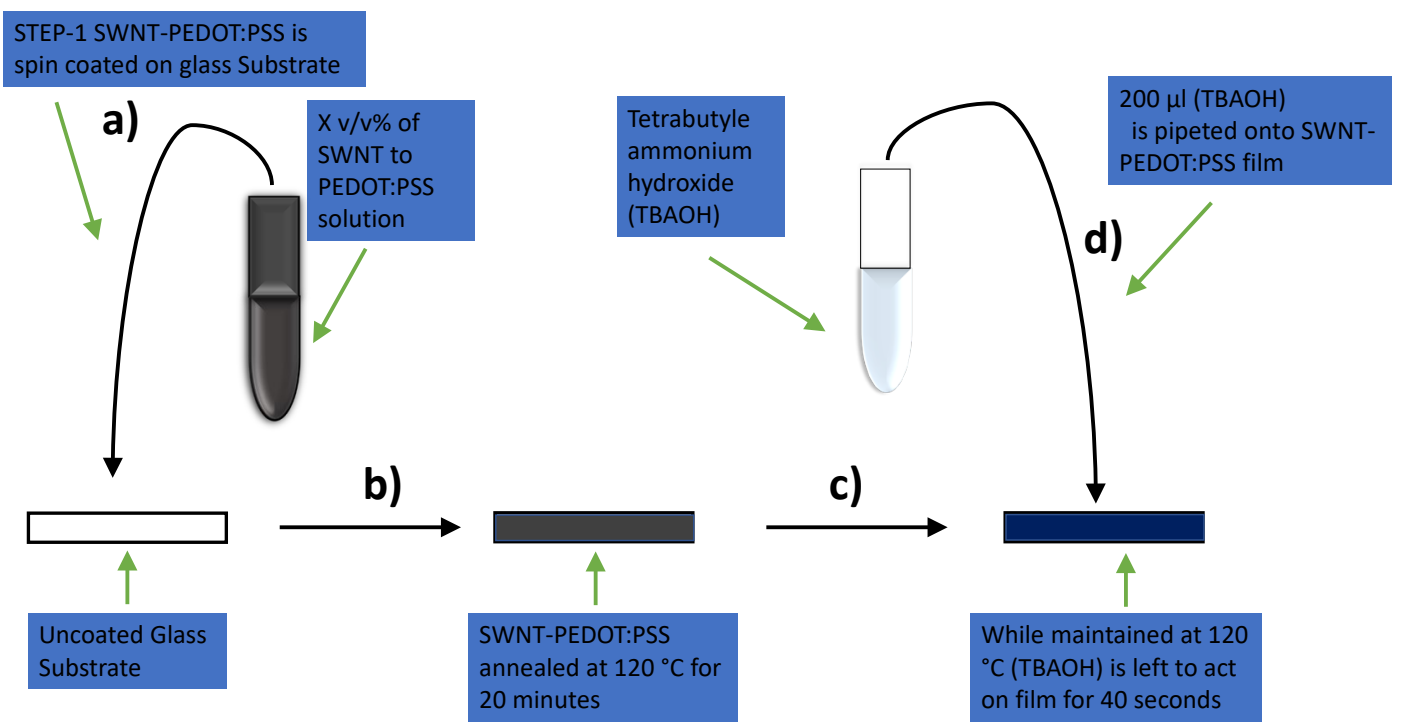

Figure 6.4.3.1 depicting the preparation of the concentration dependent SWNTPEDOT:PSS-conc films. X refers to a particular $v / v$ concentration. For example, the $5 \% v / v$ is $X \% v / v$.

\subsection{Results and discussion}

\subsubsection{Preliminary experimental considerations}

Figure 6.5.1.1 depicts the thermoelectric properties 5 out of 6 control films, namely, 1-pristine PEDOT:PSS, 2-pristine PEDOT:PSS-DMSO, 3-pristine PEDOT:PSS-DMSO-TBAOH, 4-100\% SWNT-DMSO, and 5-100\% SWNT-DMSOTBAOH. The 6-100\% SWNT films was "too" poorly conducting to yield any reliable thermoelectric measurements. It is of note that said thermoelectric properties of 
pristine PEDOT:PSS-TBAOH film ( whereby a pristine PEDOT:PSS is post treated with $\mathrm{TBAOH}$ ), could not be measured due to being "too" poorly conductive (conductivity was outside the range of measurement the ZEM-3 system could reliably elucidate). It was also observed that the Seebeck coefficient could not be reliably determined either.

As shown in Figure 6.5.1.1 (a) the electrical conductivity of 1-pristine PEDOT:PSS film is (3-4 $\left.\mathrm{S} \mathrm{cm}^{-1}\right)$ meanwhile the 2-pristine PEDOT:PSS-DMSO film is $\left(800 \mathrm{~S} \mathrm{~cm}^{-1}\right)$ as observed in literature. ${ }^{179,299,337}$ Both films exhibit a positive Seebeck coefficient (indicating p-type behaviour) in the same range $\left(12-14 \mu \mathrm{V} \mathrm{K}^{-1}\right.$ ) as expressed in Figure 6.5.1.1 (b)., ${ }^{299,337,397}$ the 3-pristine PEDOT:PSS-DMSO-TBOAH film has an electrical conductivity of $3.4 \mathrm{~S} \mathrm{~cm}^{-1}$ at $40{ }^{\circ} \mathrm{C}$. The $4-100 \%$ SWNT-DMSO has a moderately high electrical conductivity of $500 \mathrm{~S} \mathrm{~cm}^{-1}$ at the same temperature. The film's Seebeck coefficient (Figure 6.5.1.1 (b), is relatively high at $27 \mu \mathrm{V} \mathrm{K}^{-1}$ (positive sign indicating p-type characteristics) ${ }^{136,161,319}$ at the same temperature. The 5-100\% SWNT-DMSO-TBAOH however, has a negative Seebeck coefficient (indicating n-type characteristics) ${ }^{398,399}$ of $-25 \mu \mathrm{V} \mathrm{K}^{-1}$ at $40^{\circ} \mathrm{C}$. The film has the highest electrical conductivity of $1750 \mathrm{~S} \mathrm{~cm}^{-1}$ at $40{ }^{\circ} \mathrm{C}$ reaching $2250 \mathrm{~S} \mathrm{~cm}^{-1}$ at $110{ }^{\circ} \mathrm{C}$. In conjunction with electrical conductivity the power factor (Figure 6.5.1.1 (c) is $96 \mu \mathrm{W}$ $\mathrm{m}^{-1} \mathrm{~K}^{-2}$ at $40^{\circ} \mathrm{C}$.

As shown from Figure 6.5.1.1 (c) the power factor corresponding to 1-pristine PEDOT:PSS and 3-pristine PEDOT:PSS-DMSO-TBAOH is extremely low $(<0.17$ $\mu \mathrm{W} \mathrm{m}^{-1} \mathrm{~K}^{-2}$ ) at $40{ }^{\circ} \mathrm{C}$. The 2-pristine PEDOT:PSS-DMSO and 4-100\% SWNT-DMSO film have a significantly higher power factor of $12 \mu \mathrm{W} \mathrm{m}{ }^{-1} \mathrm{~K}^{-2}$ and $38 \mu \mathrm{W} \mathrm{m} \mathrm{K}^{-1} \mathrm{~K}^{-2}$ respectively at $40{ }^{\circ} \mathrm{C}$.

Due to the pristine PEDOT:PSS film treated with TBAOH having a significantly reduced electrical conductivity that led to unreliable electrical conductivity and Seebeck coefficient measurements by the ZEM-3 system, DMSO was used to treat all SWNT-PEDOT:PSS-conc and SWNT-PEDOT:PSS-cont films to improve initial conductivity high enough that after TBAOH it would still be measurable as indicated by the thermoelectric properties of the (3-pristine PEDOT:PSS-DMSO-TBAOH) film.

The preliminary experiments involved using the $\left(1 \% \mathrm{w} / \mathrm{v} \mathrm{NaBH}_{4}-\mathrm{DMSO}\right)$ solution utilized in chapter 5 to treat the PEDOT:PSS-ionic liquid composites on the SWNT-PEDOT:PSS films however n-type characteristics were not observed 
consistently. TBAOH was selected as treatment agent due to producing n-type characteristics on SWNT-PEDOT:PSS films consistently after several repeats.

a)

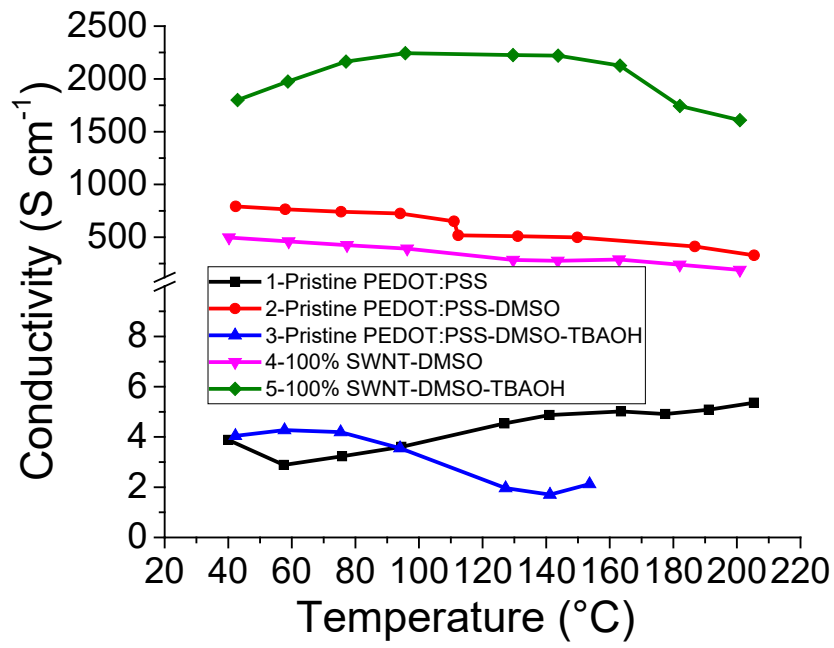

b)
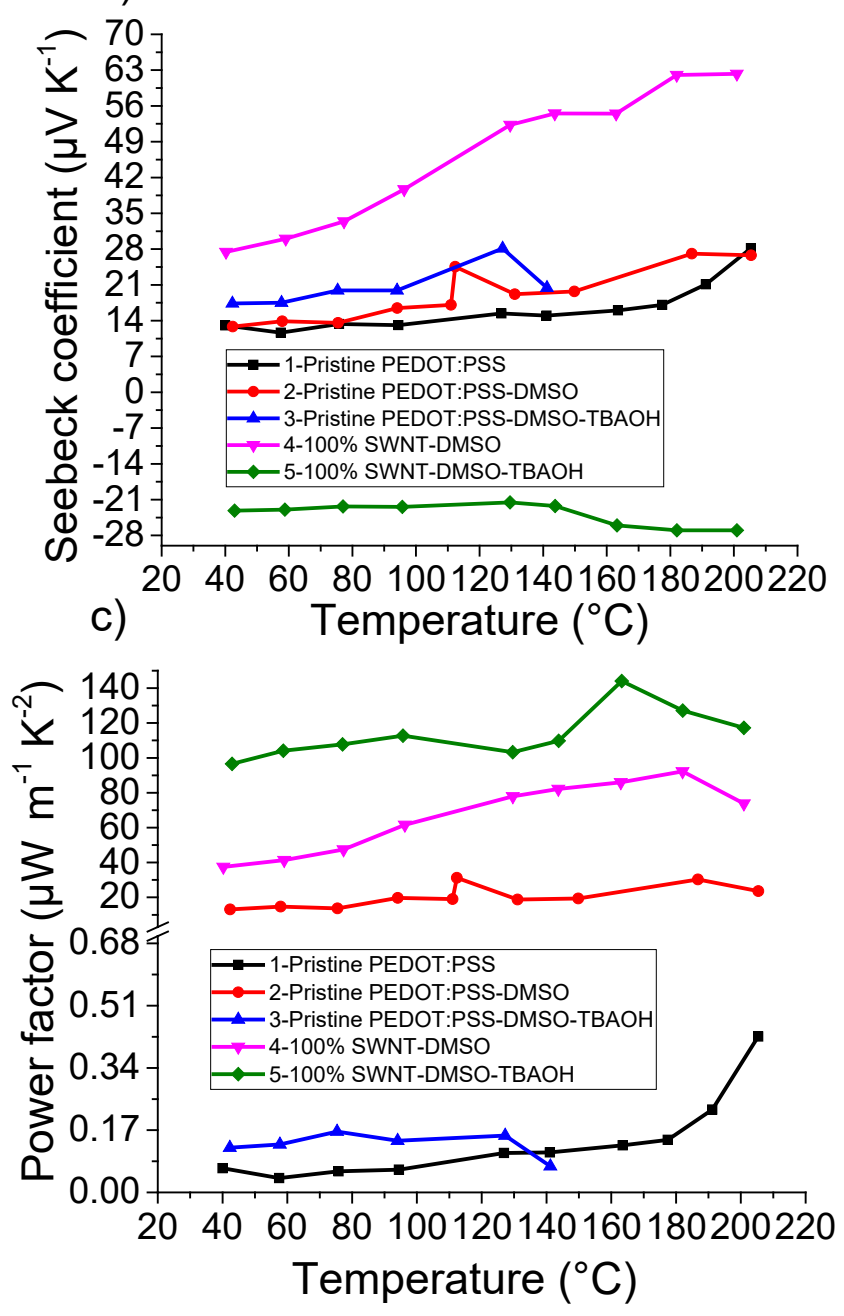
Figure 6.5.1.1 The thermoelectric properties 5 of possible 6 controls. (a), Depicts the electrical conductivity against temperature, meanwhile (b) represents the Seebeck coefficient against increasing temperature and (c) the Power factor against an increasing temperature profile.

\subsubsection{Thermoelectric properties of SWNT-PEDOT:PSS-cont films}

Figure 6.5.2.1 as shown below represents the electrical conductivity of SWNTPEDOT:PSS composites (internally treated with 5\% v/v DMSO). As expressed in the Figure 6.5.2.1 all films depicted in (a) (SWNT-PEDOT:PSS-cont) have significantly higher electrical conductivity than those in (b) (SWNT-PEDOT:PSS-conc). As shown in Figure 6.5.2.1 (a) the film with the highest electrical conductivity is the $80 \%$ SWNT-PEDOT:PSS-cont whereby at $40{ }^{\circ} \mathrm{C}$ the electrical conductivity is $1760 \mathrm{~S} \mathrm{~cm}^{-}$ 1. The high electrical conductivity can be explained by synergistic effects after DMSO is involved in partial PSS removal causing a reorientation of PEDOT chains from coiled shell structures to more expanded coil and linear structures and the subsequent improved $\pi-\pi$ stacking between PEDOT chains and SWNTs. ${ }^{400-402}$

The film with the second highest electrical conductivity Figure 6.5.2.1 (a) is the $40 \%$ SWNT-PEDOT:PSS-cont film at $40{ }^{\circ} \mathrm{C}$. At higher temperatures however, the film's electrical conductivity intersects with the $10 \%$ SWNT-PEDOT:PSS-cont film which in from $80^{\circ} \mathrm{C}$ has a higher temperature than the $40 \%$ SWNT-PEDOT:PSScont film. As was discussed in chapter 4, the idea of (non-thermally assisted hopping $)^{132,220,257}$ was used described electrical conductivities that were not improved by temperature. In this cause the SWNT-PEDOT:PSS-cont films do not exhibit positive thermal dependence on the electrical conductivity. In fact, (except for the $10 \%$ SWNT-PEDOT:PSS-cont) as the temperature increases the electrical conductivity decreases for the remaining films depicted in Figure 6.5.2.1 (a). In Figure 6.5.2.1 (a) the two films with the lowest electrical conductivities are the $5 \%$ SWNTPEDOT:PSS-cont and $20 \%$ SWNT-PEDOT:PSS-cont . Although the two films with the highest SWNT concentration had the highest electrical conductivity, films with lower than $40 \%$ SWNT content appear to not follow a concentration dependent electrical conductivity pattern. This may be because there could be minimum SWNT 
concentration needed to build effective $\pi-\pi$ bridges between itself and disconnected PEDOT chains. ${ }^{401}$

The electrical conductivity of the films of interest (treated with TBAOH) are shown in Figure 6.5.2.1 (b). There is no consistent carrier mechanism that describes all films (thermally or non-thermally assisted hopping). This is because some films such as (80\% SWNT-PEDOT:PSS-conc) exhibit improved electrical conductivity with increased temperature meanwhile some exhibit the inverse trend (5\% SWNTPEDOT:PSS-conc). 


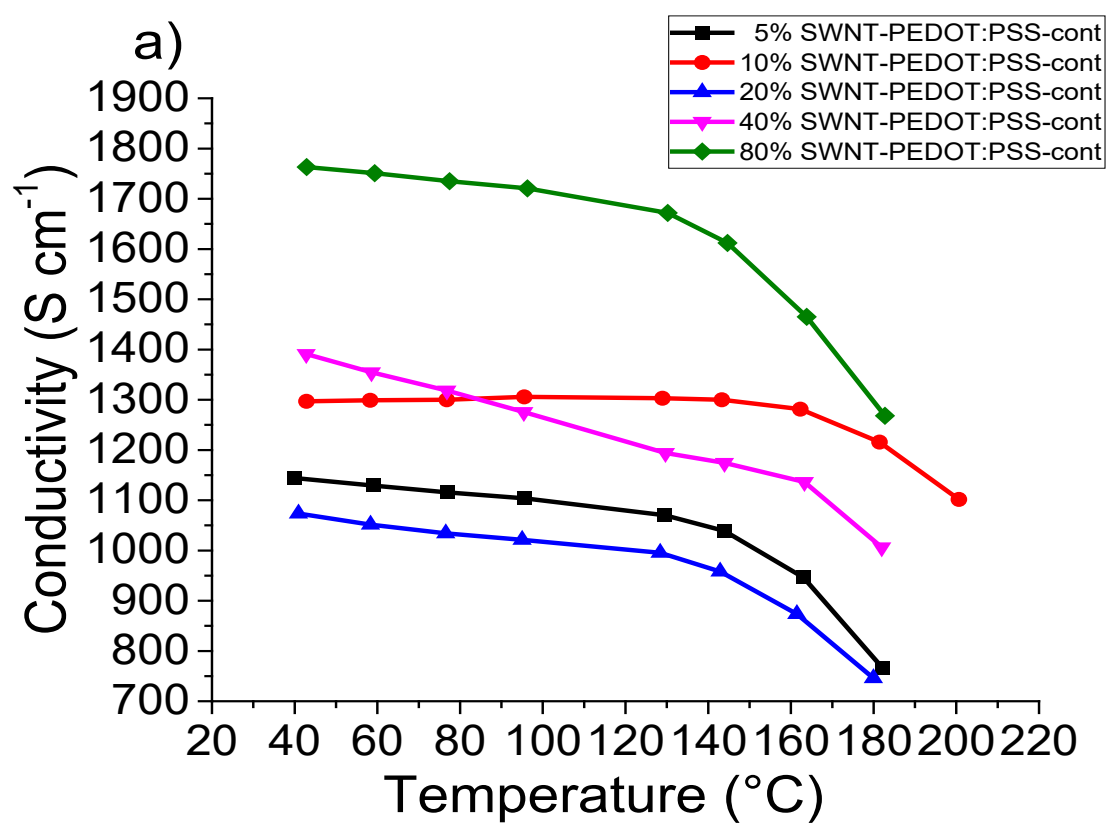

b)

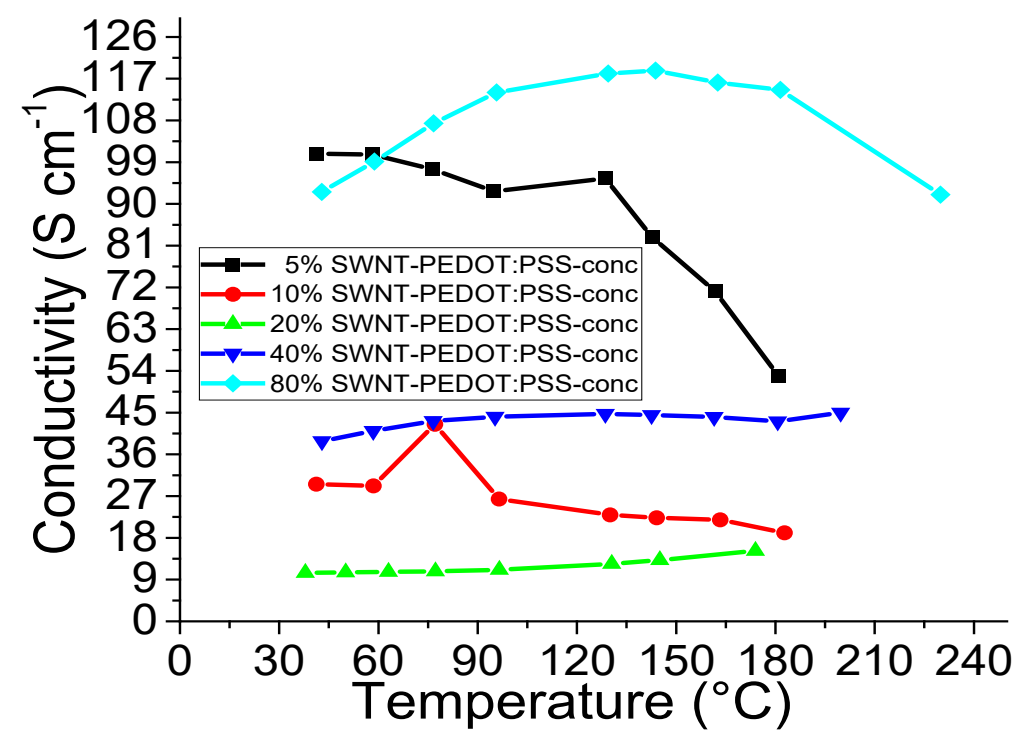

Figure 6.5.2.1 Represents the electrical conductivity against a temperature profile for the SWNT-PEDOT:PSS films. (a) Depicts the SWNT-PEDOT:PSS-cont films (without TBAOH) treatment meanwhile (b) depicts the SWNT-PEDOT:PSS-conc films (with TBAOH) treatment.

The $5 \%$ SWNT-PEDOT:PSS-conc showed the highest electrical conductivity for the set of films $\left(100 \mathrm{~S} \mathrm{~cm}^{-1}\right)$ at $40{ }^{\circ} \mathrm{C}$. However, across the whole temperature profile the $80 \%$ SWNT-PEDOT:PSS-conc film had a much higher electrical conductivity which peaked as $\left(120 \mathrm{~S} \mathrm{~cm}^{-1}\right)$ at $150{ }^{\circ} \mathrm{C}$. The film with the lowest electrical conductivity was the $20 \%$ SWNT-PEDOT:PSS-conc which at $40^{\circ} \mathrm{C}$ was 10 
$\mathrm{S} \mathrm{cm}^{-1}$ which although higher than that of the 1-pristine PEDOT:PSS film as shown in Figure 6.5.1.1 (a) did not increase significantly across the temperature profile.

Figure 6.5.2.2 shows the Seebeck coefficient across a temperature profile $40{ }^{\circ} \mathrm{C}$ - 240 ${ }^{\circ} \mathrm{C}$ for (a) SWNT-PEDOT:PSS-cont and (b) SWNT-PEDOT:PSS-conc films. All films shown in Figure 6.5.2.1 (a), exhibit a p-type characteristic as confirmed by the positive Seebeck coefficient. ${ }^{161,319,403}$ There appears however, to be no complete relationship between the concentration of the SWNT in the film and the corresponding value of the Seebeck coefficient. The $5 \%$ SWNT-PEDOT:PSS-cont film has the lowest Seebeck coefficient ( $<9 \mu \mathrm{V} \mathrm{K}^{-1}$ ) however the $10 \%$ SWNT-PEDOT:PSS-cont film is shown to have the highest Seebeck coefficient ( $>16 \mu \mathrm{V} \mathrm{K}^{-1}$ ) with the $80 \%$ and $40 \%$ SWNTPEDOT:PSS-cont films having a slightly lower values of Seebeck coefficients between $\left(13-14 \mu \mathrm{V} \mathrm{K}^{-1}\right)$ at $40{ }^{\circ} \mathrm{C}$. This is unlike the electrical conductivity trend for the films whereby there was some relationship between the concentration of SWNT and the electrical conductivity especially for the higher concentrations as shown in Figure 6.5.2.1 (a). For the SWNT-PEDOT:PSS-conc films however as depicted in Figure 6.5.2.2 (b) it is clear that some films exhibit n-type ${ }^{399}$ characteristics. 3 SWNTPEDOT:PSS-conc films expressed a negative Seebeck coefficient. It is of note that the films with n-type characteristics at $40^{\circ} \mathrm{C}$ were the 3 films with the highest SWNT concentrations. Namely, the 20, 40 and $80 \%$ SWNT-PEDOT:PSS-conc films. The 40\% SWNT-PEDOT:PSS-conc exhibited the largest in magnitude negative Seebeck coefficient $\left(-38 \mu \mathrm{V} \mathrm{K}^{-1}\right)$ relative to any film in the study. The 80 and $20 \%$ SWNTPEDOT:PSS-conc films had lower Seebeck coefficients of $24 \mu \mathrm{V} \mathrm{K}^{-}$and $28 \mu \mathrm{V} \mathrm{K}^{-1}$. From the results it appears that under the treatment conditions $20 \% \mathrm{SWNT} \mathrm{v} / \mathrm{v}$ to PEDOT:PSS solution is required to induce n-type characteristics. The Seebeck coefficient is competitive with values obtained from literature on SWNT doping. ${ }^{295,404}$ Interestingly there is a sign change from $\mathrm{p}$ - to $\mathrm{n}$ - type that is temperature dependent for the 10\% SWNT-PEDOT:PSS-conc film. The film's Seebeck coefficient is $<9 \mu \mathrm{V} \mathrm{K}{ }^{--}$ ${ }^{1}$ at $40{ }^{\circ} \mathrm{C}$ meanwhile at $75{ }^{\circ} \mathrm{C}$ it is $-1 \mu \mathrm{V} \mathrm{K}^{-1}$. The Seebeck coefficient of the film continues to become larger in magnitude and remains negative across temperature range and peaks as $-16 \mu \mathrm{V} \mathrm{K}^{-1}$ at $195^{\circ} \mathrm{C}$. The 3 films that had an electrical conductivity showing some thermal dependence (thermally assisted hopping) mechanism as seen in Figure 6.5.2.1 (b) were also the films with the n-type Seebeck coefficient at $40{ }^{\circ} \mathrm{C}$.

\section{$131 \mid \mathrm{P}$ a g e}


a)

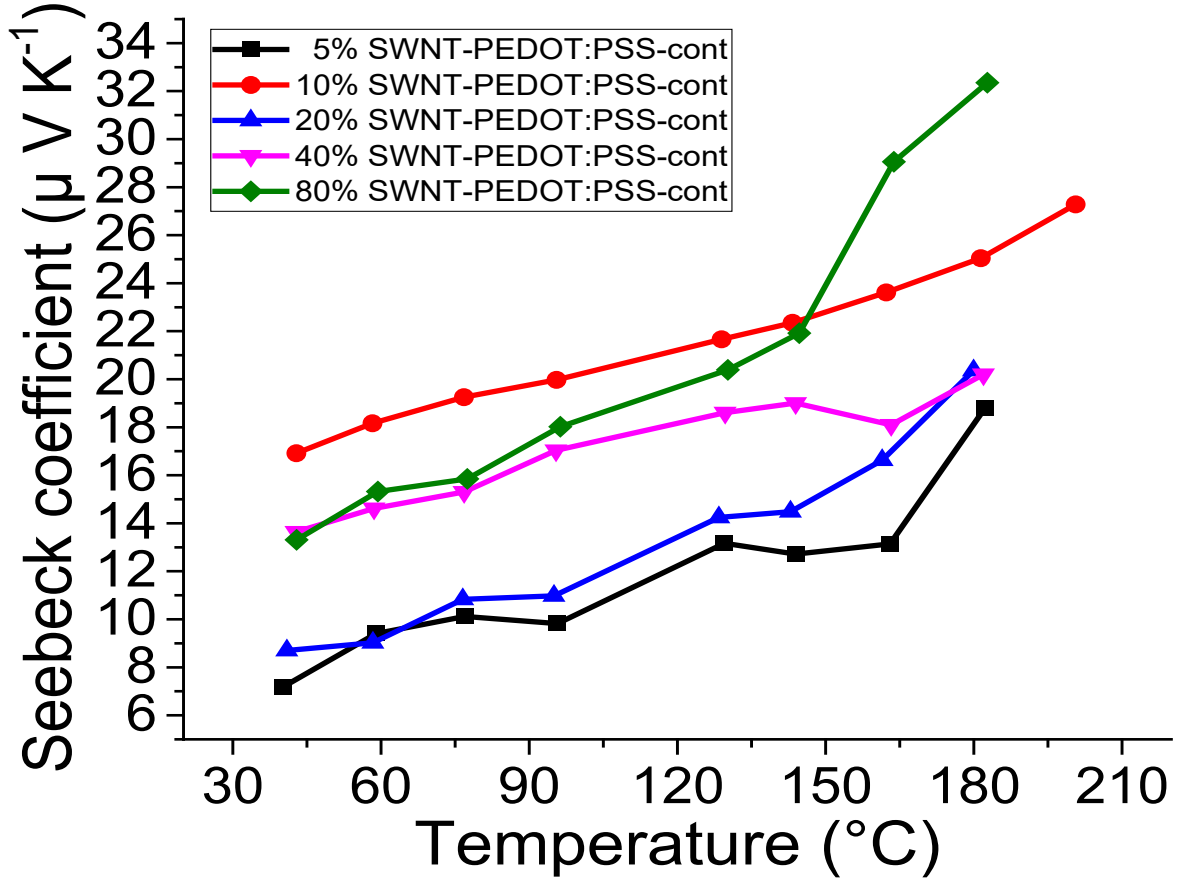

b)

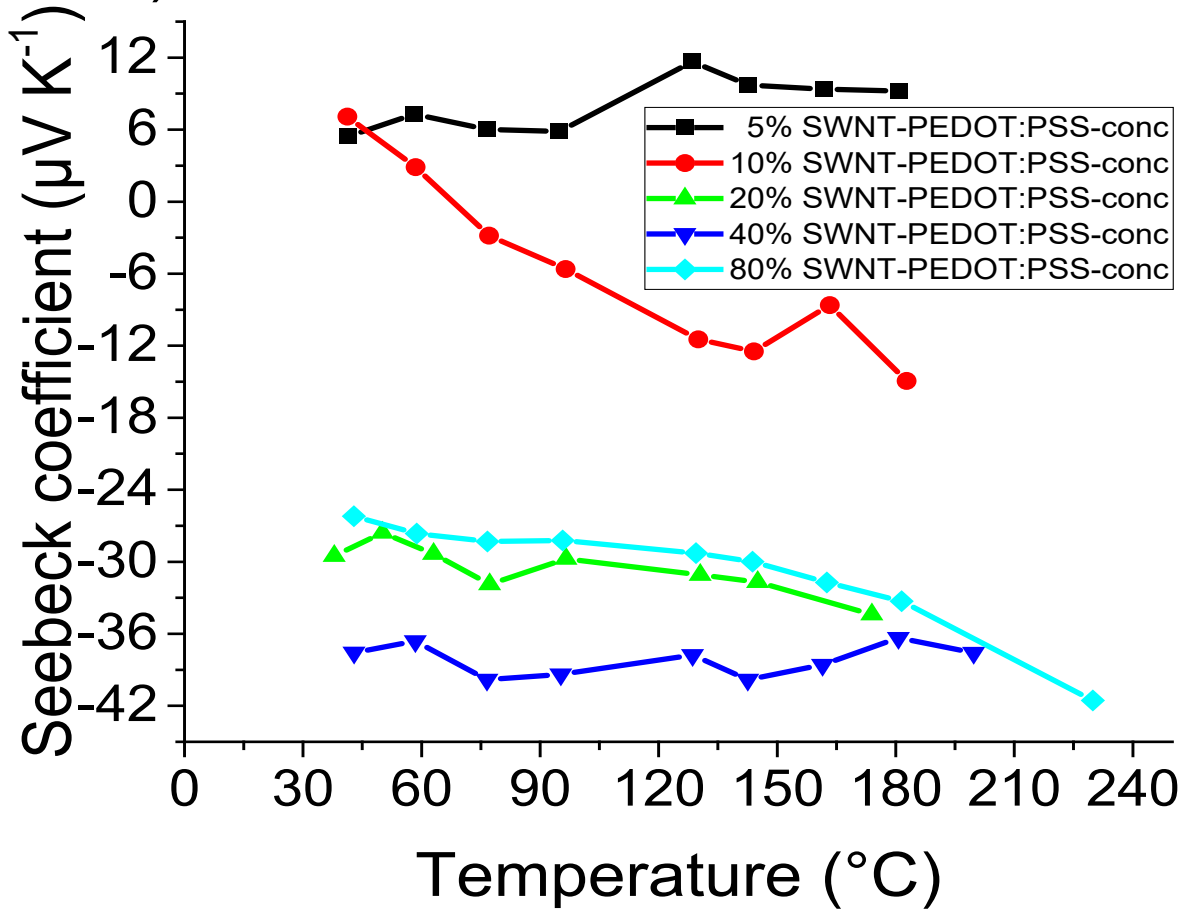

Figure 6.5.2.2 depicting the Seebeck coefficient across a temperature range $40{ }^{\circ} \mathrm{C}$ $240{ }^{\circ} \mathrm{C}$, for (a) the SWNT-PEDOT:PSS-cont (not TBAOH treated) and (b) the SWNTPEDOT:PSS-conc (TBAOH treated) films. 
Because the 3-pristine PEDOT:PSS-DMSO-TBAOH film (Figure 6.5.1.1 (b) did not develop n-type characteristics yet the 5-100\% SWNT-DMSO-TBAOH film did as shown by the negative Seebeck coefficient, in conjunction with the n-type characteristics present in the SWNT-PEDOT:PSS-conc films(Figure 6.5.2.2 (b), it can be deduced that under the treatment conditions (chapter 6.4) that TBAOH is indeed an $\mathrm{n}$ dopant. It is also conclusive that SWNT presence is required to make the PEDOT films n-type. In a recent study ${ }^{405}$ however, it was shown than a PEDOT only n-type thermoelectric film could be synthesized. It was however shown that the film had an extremely low electrical conductivity $\left(0.01 \mathrm{~S} \mathrm{~cm}^{-1}\right)$ and Seebeck coefficient of $<-20$ $\mu \mathrm{V} \mathrm{K}^{-1} .{ }^{405}$ The Seebeck coefficient $\left(-26 \mu \mathrm{V} \mathrm{K}^{-1}\right)$ and electrical conductivity $91 \mathrm{~S} \mathrm{~cm}^{-1}$ at $40{ }^{\circ} \mathrm{C}$ for the $80 \%$ SWNT-PEDOT:PSS-conc film is more negative.

Figure 6.5.2.3 shows the power factor against the temperature it is measured at for the SWNT-PEDOT:PSS-cont (a), and SWNT-PEDOT:PSS-conc (b) films. As is evident almost all the SWNT-PEDOT:PSS-cont films have significantly higher power factors at $40{ }^{\circ} \mathrm{C}$ and across the temperature range than the SWNT-PEDOT:PSS-conc films. For the SWNT-PEDOT:PSS-cont films there is a general upward trend for the power factor as the temperature increases. A similar trend is observed for the SWNTPEDOT:PSS-conc films, except for the 40\% SWNT-PEDOT:PSS-conc film which has a power factor that is largely unchanged across the measured temperature range.

The SWNT-PEDOT:PSS-cont films with the lowest power factor relative to the other films in Figure 6.5.2.3 (a) are 5 and 20\% SWNT-PEDOT:PSS-cont. The power factor for said films resemble the one observed for the $80 \%$ SWNT-PEDOT:PSS-conc film (Figure 6.5.2.3 (b). Because the electrical conductivity was highest for the $80 \%$ SWNT-PEDOT:PSS-cont film followed by the 10 and 40\% SWNT-PEDOT:PSS-cont (Figure 6.5.2.1 (a) it is not surprising that the 80\% SWNT-PEDOT:PSS-cont had the highest power factor for majority of the measured temperature range. It is also of note that the Seebeck coefficient resembles the power factor almost exactly in terms of the order of films. This is because except for the 80\% SWNT-PEDOT:PSS-cont film's electrical conductivity the remaining of the SWNT-PEDOT:PSS-cont films had an electrical conductivity in a similar range of each other. Although the SWNTPEDOT:PSS-cont films acted as controls to compare with the TBAOH treated films 
(SWNT-PEDOT:PSS-conc) films for understanding the origins of n-type characteristics, the (SWNT-PEDOT:PSS-cont) films showed high potential as OHTEGs due to their excellent power factors. The 80\% SWNT-PEDOT:PSS-cont film in particular had a high-power factor of $36 \mu \mathrm{W} \mathrm{m} \mathrm{K}^{-1}$ at $40{ }^{\circ} \mathrm{C}$. Compared to the 1pristine PEDOT:PSS $\left(<0.07 \mu \mathrm{W} \mathrm{m}^{-1} \mathrm{~K}^{-2}\right)$ and 5\% DMSO treated PEDOT:PSS film $\left(<14 \mu \mathrm{W} \mathrm{m}^{-1} \mathrm{~K}^{-2}\right)$ as shown in Figure 6.5.1.1 (c) the 80\% SWNT-PEDOT:PSS-cont film showed greater thermoelectric performance. The 80\% SWNT-PEDOT:PSS-conc had a power factor of around $7 \mu \mathrm{W} \mathrm{m}^{-1} \mathrm{~K}^{-2}$ at $40{ }^{\circ} \mathrm{C}$ meanwhile the $40 \%$ SWNTPEDOT:PSS-conc had a power factor of $5 \mu \mathrm{W} \mathrm{m} \mathrm{m}^{-1} \mathrm{~K}^{-2}$ at the same temperature. When compared to a recent study claiming first n-type PEDOT nanoparticle film with a power factor of $0.0001805 \mu \mathrm{W} \mathrm{m} \mathrm{m}^{-1} \mathrm{~K}^{-2}$ for a $0.1 \mathrm{M}$ dodecyltrimethylammonium bromide (DTAB) treatment the films produced in this chapter were significantly better 
perfoming. ${ }^{405}$

a)

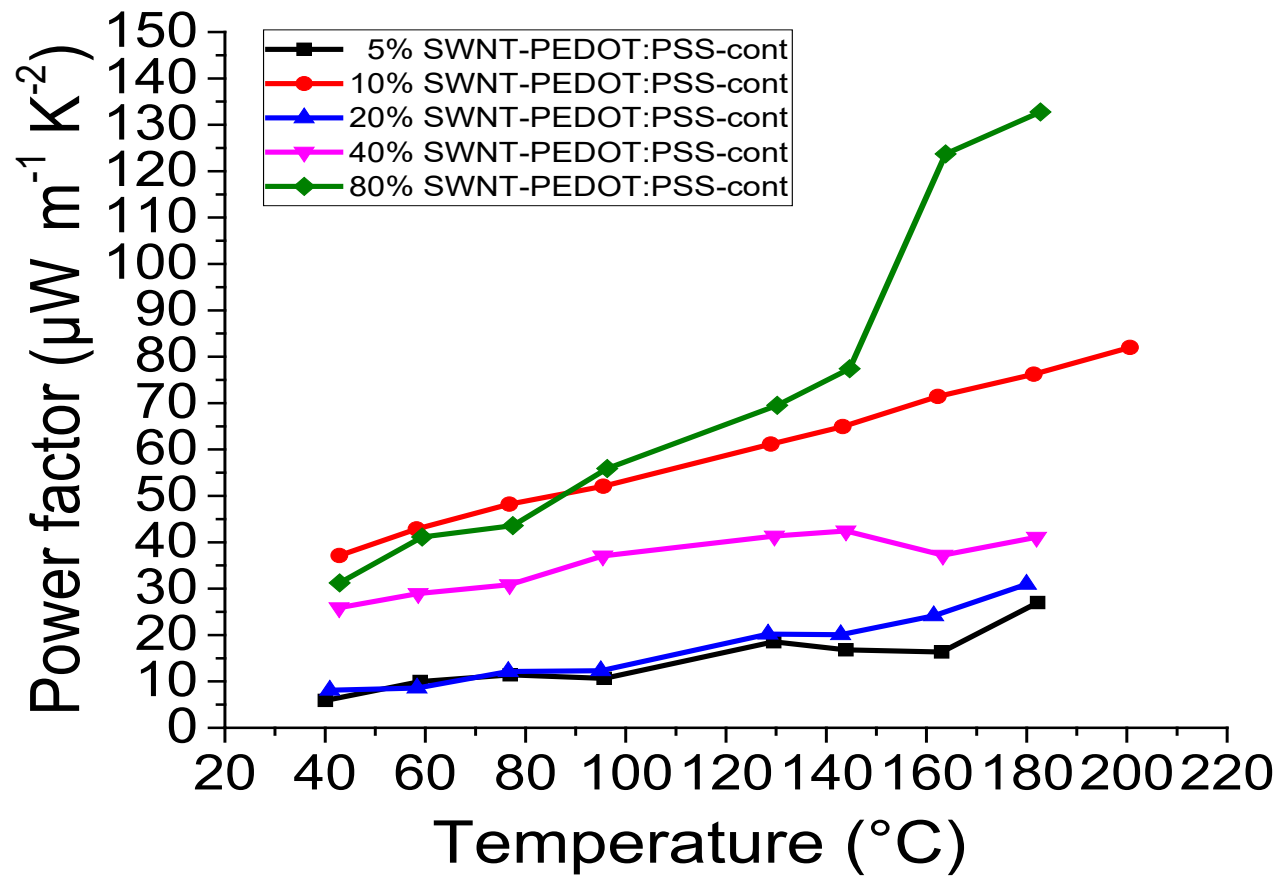

b)

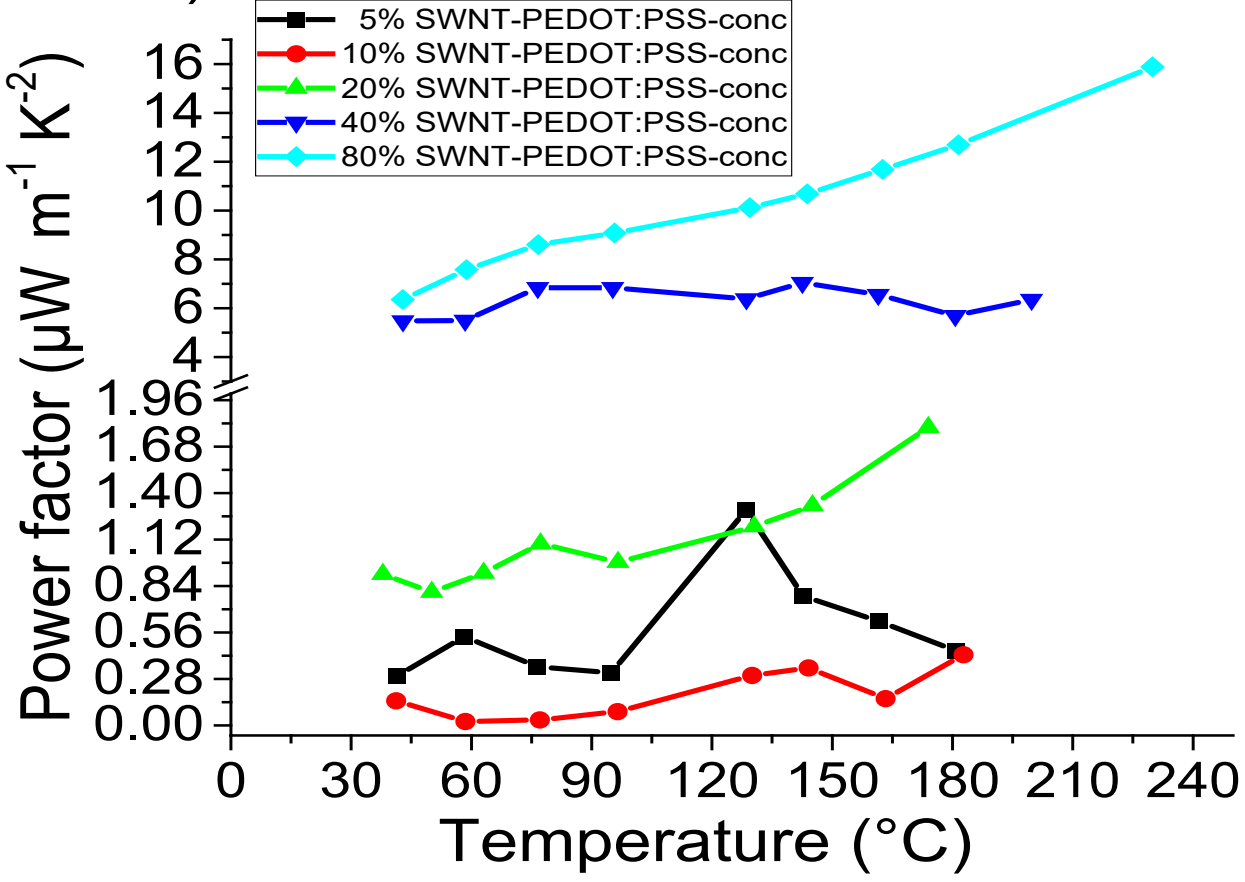


Figure 6.5.2.3 The Power factor across the temperature range of $40^{\circ} \mathrm{C}$ - $240{ }^{\circ} \mathrm{C}$ for the SWNT-PEDOT:PSS-cont films (a) (without TBAOH treatment), and for the SWNTPEDOT:PSS-conc films (b) (with TBAOH treatment).

\subsection{Doping mechanism behind N-type characteristics of SWNT-PEDOT:PSS}

Raman spectroscopy can give useful information regarding the oxidation state of thiophene molecules in PEDOT:PSS as well as indicate the conformation of majority of thiophene molecules (benzoid or quinoid). ${ }^{298,363,406}$ Because of the sensitivity of Raman spectroscopy, it's possible to detect changes in carrier concentration of SWNT via the position and shift of the G band (typically found around $1590 \mathrm{~cm}^{-1}$ depending on sample or chemical environment). ${ }^{137,295}$ This means Raman studies can be utilized to corroborate n-type doping via red shift of the $G$ band. ${ }^{295}$ Figure 6.6.1 (a) shows the Raman active stretching vibrational regions for 3 control PEDOT:PSS samples. The Raman shift at $1365 \mathrm{~cm}^{-1}, 1434 \mathrm{~cm}^{-1}$ and $1505 \mathrm{~cm}^{-}$ ${ }^{1}$ depict the PEDOT inter ring $\left(\mathrm{C}_{\beta}-\mathrm{C}_{\beta}\right)$, symmetrical $\left(\mathrm{C}_{\alpha}=\mathrm{C} \beta\right)$ and asymmetrical $\mathrm{C}_{\alpha}=$ $\mathrm{C}_{\beta}$ stretching vibrations respectively. ${ }^{308,397,406}$ Figure 6.6.1 (b) depicts the Raman shift at the $\mathrm{G}$ band $\left(1590 \mathrm{~cm}^{-1}\right)$ for spectra $3 \mathrm{SWNT}$ control samples.

As exhibited in Table 6.6.1 and shown in Figure 6.1.1 (a) the 1-pristine PEDOT:PSS film (utilized as a reference) has a symmetrical $\mathrm{C}_{\alpha}=\mathrm{C}_{\beta}$ stretching at 1434 $\mathrm{cm}^{-1}$ whereby relative to the other 2 films it's the most blue shifted. ${ }^{298,365,372}$ The 2 pristine PEDOT:PSS-DMSO sample $\left(1430 \mathrm{~cm}^{-1}\right)$ is slightly red shifted and the TBAOH treated (3-pristine PEDOT:PSS-DMSO-TBAOH) film exhibits the most significant red shift $\left(1425 \mathrm{~cm}^{-1}\right)$. The red shifts for the aforementioned samples indicate that both the DMSO treatment and the TBAOH treatment causes some quantity of thiophene molecules to undergo conformational change from benzoid to quinoid whereby the intra chain transport is more ordered due to better $\pi-\pi$ stacking (please see Figure 6.6 .2 (b)/(a). ${ }^{300,337,357,361,372}$ The extent of the red-shift for the TBAOH treated film is more significant $\left(1425 \mathrm{~cm}^{-1}\right)$ than the purely DMSO treated film. As explained in chapter 5.3.4 this is due to the reducing agent indicating reduction of thiophenes. ${ }^{2}$ This is supported by the fact that the width of the peak at the symmetrical $\mathrm{C}_{\alpha}=\mathrm{C}_{\beta}$ region corresponding to the 3-pristine PEDOT:PSS-DMSOTBAOH has more narrow peaks (that are more intense but not shown due to $136 \mid \mathrm{P}$ a g e 
normalized intensities). ${ }^{209,407,408}$ This has been coupled with a blue shifted asymmetrical $\mathrm{C}_{\alpha}=\mathrm{C}_{\beta}$ from around $1505 \mathrm{~cm}^{-1}$ (1-pristine PEDOT:PSS and 2-pristine PEDOT:PSS-DMSO) to $1512 \mathrm{~cm}^{-1}$ for the 3-pristine PEDOT:PSS-DMSO-TBAOH as also observed in literature. ${ }^{409}$

As indicated by the reduction this suggests that some thiophene molecules in response to $\mathrm{TBAOH}$ treatment are reduced to at least the polaron $\left(\mathrm{PEDOT}^{+}\right.$state from the bipolaron PEDOT $^{2+}$ state). Please refer to Figure 6.6.2 (d)/(c). Without UV-Vis data to corroborate the (extent of reduction) it is not possible to know if the films were reduced to the Neutral state $\left(\mathrm{PEDOT}^{\mathrm{O}}\right.$ ) (please refer to Figure 6.6.2 (a).
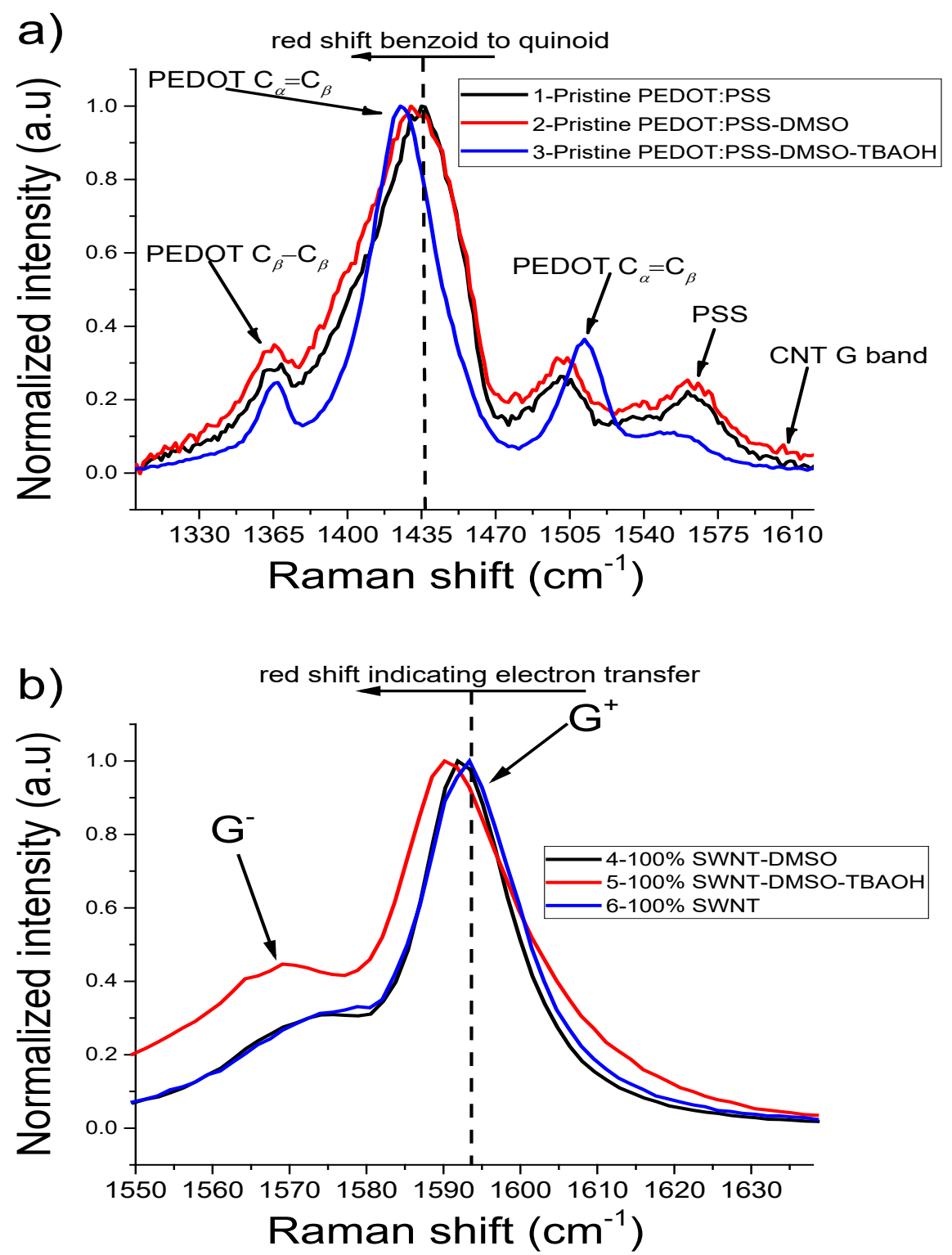
Figure 6.6.1 depicting the Raman spectra for control films labelled with a numerical prefix between 1 and 6. (a) Depicts Raman shift at the benzoid to quinoid region $\left(C_{a}\right.$ $=C \beta)$ for PEDOT:PSS based films meanwhile (b) represents Raman shift at the $G$ band for SWNT based films.

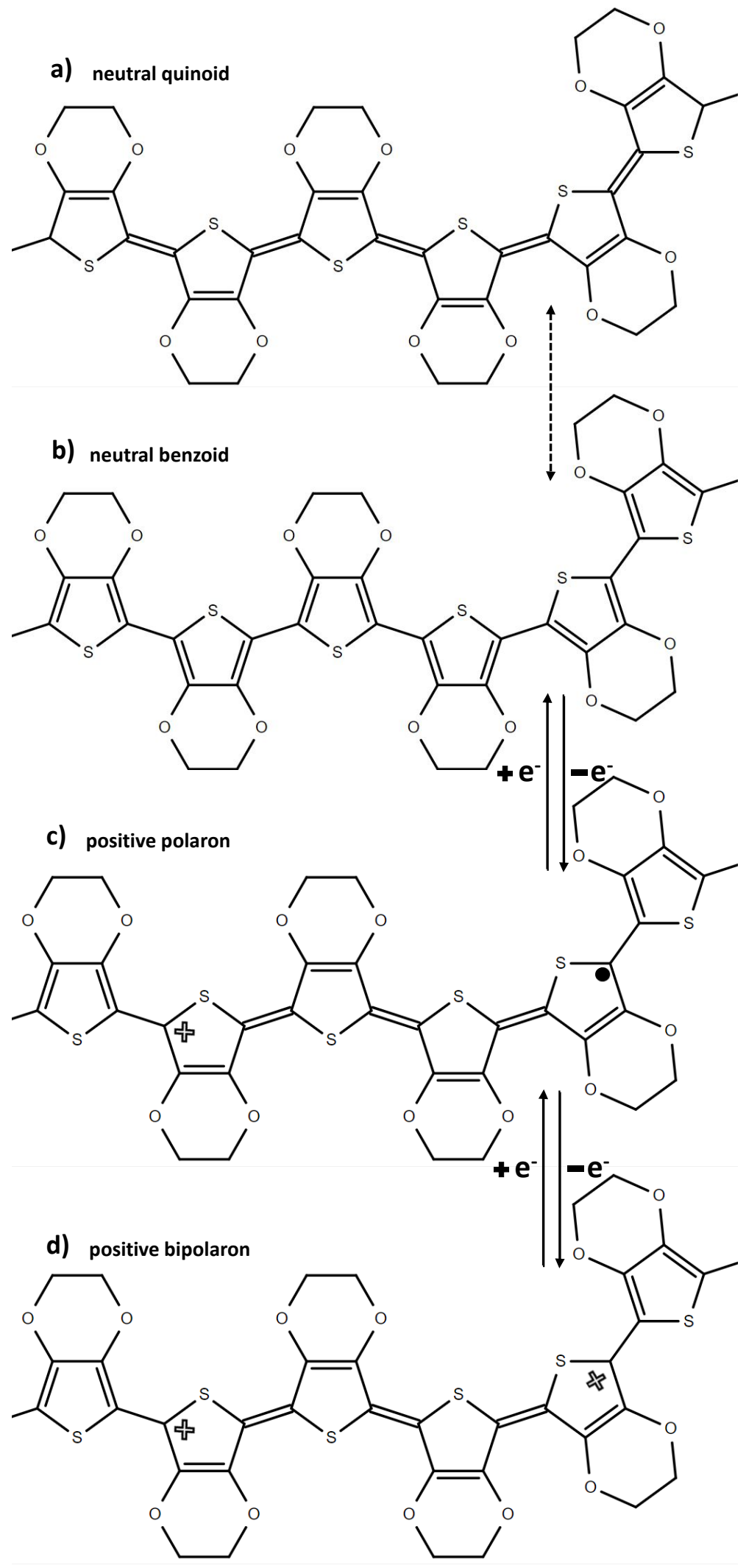


Figure 6.6.2 The resonance forms of Poly(3,4-ethylenedioxythiophene) (PEDOT) thiophene back bone, (a) denotes the quinoid form, meanwhile (b) is the benzoid form. (c) and (d) represent the positive polaron and bipolaron phase, respectively.

It was not possible to obtain UV-Vis-NIR data due to COVID-19 lockdown of the University. Still, it is thought that the films were indeed reduced to the neutral phase as was inferred by the deep blue colour observed during reducing treatment as well as the inability to obtain reliable electrical conductivity measurements on the TBAOH treated pristine PEDOT:PSS.

Figure 6.6.1 (b) shows Raman shift for the $\mathrm{G}^{+}$band at $1593 \mathrm{~cm}^{-1}$ for the 6$100 \%$ SWNT. Because the SWNTs were poorly conducting, it was not possible to get "reliable" thermoelectric measurements utilizing ZEM-3. Still it is known that the SWNTs used were p-type due to the positive sign Seebeck coefficient sign of the 4100\% SWNT-DMSO (Figure 6.5.11 (b) and the SWNT-PEDOT:PSS-cont films (Figure 6.5.2.2 (a). A red shift is observed from $1593 \mathrm{~cm}^{-1}$ to $1592 \mathrm{~cm}^{-1}$ with DMSO treatment for 4-100\% SWNT-DMSO. DMSO is not usually known to inject electrons into SWNT therefore this will need to be explored in future studies. The 5-100\% SWNT-DMSO-TBAOH films exhibits a further red shift to $1590 \mathrm{~cm}^{-1}$ thereby indicating electron transfer to the SWNTs thus explaining n-type characteristics observed in the Seebeck coefficient (Figure 6.5.1.1 (b). ${ }^{410}$ It was discovered that the thermoelectric properties of the 5-100\%-SWNT-DMSO-TBAOH film improved significantly with a corresponding sign change to negative Seebeck coefficient $(-25 \mu \mathrm{V}$ $\mathrm{K}^{-1}$ ) as expressed in Figure 6.5.1.1 (b) and (a) and electrical conductivity of $1750 \mathrm{~S}$ $\mathrm{cm}^{-1}$ at $40{ }^{\circ} \mathrm{C}$.

Figure 6.6.3 (a) depicts the Raman spectra for the 3 SWNT samples meanwhile (b) depicts the SWNTs 2D band for the 3 SWNT samples. As shown in Figure 6.6.3 (a) the G to D ratio is extremely high thereby indicating that the SWNT utilized in this study were of high quality with few defects. After the TBAOH treatment as shown for the $5-100 \%$ SWNT-DMSO-TBAOH film the D band exhibits a red shift like the $\mathrm{G}^{+}$ band for the same film and increased D band intensity. The increased D band intensity infers that there are some defects pertaining to the $\mathrm{sp}^{2}$ carbon on the SWNTs probably due to some adsorption of the $\mathrm{TBA}^{+}$cations altering the charge periodicity induced by dangling bonds along the SWNT structure as was considered in a study utilizing quaternary nitrogen for n-type doping on carbon nanotube samples. ${ }^{295}$ (Not shown), N 
1s spectrum established the presence of nitrogenous species on films treated with TBAOH).

Figure 6.6.4 represents the normalized overlaid Raman spectra for the SWNTPEDOT:PSS-conc (DMSO and, TBAOH treated), and the SWNT-PEDOT:PSS-cont (DMSO treated) films. When analysing the films, (please refer to Table 6.6.1), it is evident that the SWNT-PEDOT:PSS-conc films have width shortening (and intensity but not shown due to normalized films) at the symmetrical $\mathrm{C}_{\alpha}=\mathrm{C}_{\beta}$ vibration region with a corresponding red shift relative to the SWNT-PEDOT:PSS-cont films. The asymmetrical $\mathrm{C}_{\alpha}=\mathrm{C}_{\beta}$ stretching vibration is around $1502-1505 \mathrm{~cm}^{-1}$ for the SWNTPEDOT:PSS-cont films., ${ }^{2,372}$ There is a blue shift observed for all the SWNTPEDOT:PSS-conc films. The blue shift in conjunction with the width shortening indicates reduction (de-doping of thiophenes chains in PEDOT). ${ }^{350,368,411}$ When comparing the red shifts for the SWNT-PEDOT:PSS-cont films relative to the (baseline) 1-pristine PEDOT:PSS film it's evident that due to the DMSO treatment that it's indicated that the SWNT-PEDOT:PSS-cont films had some thiophenes undergo conformational change from benzoid to quinoid (please see Figure 6.6.2 (b)) and (a). The Raman shift for the symmetrical $\mathrm{C}_{\alpha}=\mathrm{C}_{\beta}$ is at $1435 \mathrm{~cm}^{-1}$ for the 1-pristine PEDOT:PSS. In relation to the SWNT-PEDOT:PSS-cont films, the film with the smallest shift is the $10 \%$ SWNT-PEDOT:PSS-cont film $\left(1433 \mathrm{~cm}^{-1}\right)$ meanwhile the most significant shift is attributed to the 40\% SWNT-PEDOT:PSS-cont film (1425 $\left.\mathrm{cm}^{-1}\right)$. All SWNT-PEDOT:PSS-conc films have a Raman shift at $1425 \mathrm{~cm}^{-1}$ which is more so than all the SWNT-PEDOT:PSS-cont films except for the $40 \%$ SWNTPEDOT:PSS-cont film. This in conjunction with the width shortening as well as the blue shifted asymmetrical $\mathrm{C}_{\alpha}=\mathrm{C}_{\beta}$ indicates that the SWNT-PEDOT:PSS-conc films have undergone thiophene reduction. ${ }^{350,368,411}$ Comparing the SWNT-PEDOT:PSScont films to the 1-pristine PEDOT:PSS and 2-pristine PEDOT:PSS-DMSO films the spectra have similar symmetrical $\mathrm{C}_{\alpha}=\mathrm{C}_{\beta}$ shift (please see Table 6.6.1) and a similar width at the asymmetrical $\mathrm{C}_{\alpha}=\mathrm{C}_{\beta}$ peaks. The peak position for the asymmetrical $\mathrm{C}_{\alpha}=$ $\mathrm{C}_{\beta}$ is in a similar region around $1505 \mathrm{~cm}^{-1}$. This indicates that the SWNT-PEDOT:PSScont films did not undergo thiophene reduction.

When analysing Figure 6.6.4 it is evident that there is a peak present for all films at the G band. All SWNT-PEDOT:PSS-conc films exhibit a red shift for the $\mathrm{G}^{+}$ to $1589 \mathrm{~cm}^{-1}$ relative to the $6-100 \%$ SWNT film $\left(1593 \mathrm{~cm}^{-1}\right)$. This indicates n-type 
doping as corroborated by the Seebeck coefficient for the films (Figure 6.5.2.2 (b). ${ }^{412}$ There is a similar red shift to $1589 \mathrm{~cm}^{-1}$ in relation to the SWNT-PEDOT:PSS-cont. The inference of this shift is unknown since DMSO is not typically known to inject electrons into the SWNT.

To understand the origin of the n-type doping it was evident that $\mathrm{TBAOH}$ treatment $(5-100 \%$ SWNT-DMSO-TBAOH) induced n-type characteristics as evidenced by the sign change to negative on the Seebeck coefficient (Figure 6.5.1.1 (b). The 3-pristine PEDOT:PSS-DMSO-TBAOH film did not undergo sign change to indicate n-type doping (please see Figure 6.5.1.1 (b). Therefore, it is evident that the presence of SWNTs is necessary to dope the films under the conditions utilized in this study (chapter 6.4.1). It is however noted that it has been shown in a recent paper by Collins et al proved that n-type doping on non-composited PEDOT is possible through thiophene reduction with dodecyltrimethylammonium bromide (DTAB) treatment. ${ }^{405}$ The films produced by the research study however had an extremely low electrical conductivity $\left(0.005 \mathrm{~S} \mathrm{~cm}^{-1}\right)$ for $0.1 \mathrm{M}$ dodecyltrimethylammonium bromide (DTAB) doping which decreased with higher concentration of dopant. Therefore it's noted that it's not possible to say that PEDOT films can "only" be made n-type via interaction with foreign materials such as $\mathrm{SWNT}^{234}$ or $\mathrm{Cl}^{-}$anions adsorbed on the films surface. ${ }^{141}$ Still, it was also noted in the preliminary results that it was not possible to reliably measure thermoelectric properties of a pristine PEDOT:PSS treated with TBAOH due to an extremely low electrical conductivity. In this study it was shown that electron injection into SWNT occurs whereby the increased electron density within the SWNTs interacts with PEDOT through $\pi-\pi$ interactions as evidenced by the change in the $\mathrm{G}$ band after SWNTs being composited with PEDOT (please see Table 6.6.1). Therefore, under the constraints of this study it is indicated that the PEDOT:PSS films that had n-type characteristics induced (Figure 6.4.2.2 (b) is due to both the concentration of SWNT in conjunction with reduction of thiophene molecules. The reduction of thiophene molecules reduces the carriers in the PEDOT films from PEDOT $^{2+}$ (bipolarons) to $\mathrm{PEDOT}^{+}$(polarons). Without UV-Vis-NIR data it's not possible to be conclusive however due to the significant shift towards lower BE in the Sulphur $2 \mathrm{p}_{3 / 2}$ for the SWNT-PEDOT:PSS-conc films (see Table 6.6.4) from $163.9 \mathrm{eV}$ to as low as low as $163.43 \mathrm{eV}$ for the $80 \% \mathrm{SWNT}$-PEDOT:PSS-conc film thereby leading to $\Delta \mathrm{BE}$ 
of $0.47 \mathrm{eV}$ it is suggested that SWNT-PEDOT:PSS-conc films underwent reduction to the Neutral thiophene $\left(\mathrm{PEDOT}^{\circ}\right)$. When compared 2.5\% EMIM:TFSI-NaBH 4 shown in chapter 5 Table 5.3.4.1 the shift for the 80\% SWNT-PEDOT:PSS-conc which shifted from $163.97 \mathrm{eV}$ for pristine PEDOT:PSS to $163.77 \mathrm{eV}$ thereby making a $\triangle \mathrm{BE}$ of $0.2 \mathrm{eV}$ which was indicated as $\mathrm{PEDOT}^{2+}$ to $\mathrm{PEDOT}^{+}$with the UV-Vis spectrum for the film (chapter 5 Figure 5.3.5.1).

It's thought that the SWNT-PEDOT:PSS-conc films became n-type unlike the SWNTPEDOT:PSS-cont films due to two factors, the extent of reduction to at least polaron but probably neutral phase, as well as having SWNTs in the film. The reason why the n-type characteristics induced were concentration dependent whereby only the $20 \%$ SWNT-PEDOT:PSS-conc film developed these characterises infers that the SWNT are necessary to hold or transport the negative charge. In literature it's known that PEDOT and CNTs can undergo $\pi-\pi$ interactions. ${ }^{400-402}$ Once the concentration is enough to overcome the thiophenes (p-type carriers) concentration due to adequate $\pi-\pi$ interactions between PEDOTs reduced carriers and SWNTs increased (n-type carriers) then the n-type characteristics are observed in the bulk film. This explains why the SWNT-PEDOT:PSS-cont films which did not undergo reduction as evidenced via the Raman larger width (relative to the SWNT-PEDOT:PSS-conc) films at the symmetrical $\mathrm{C}_{\alpha}=\mathrm{C}_{\beta}$ or blue shift at the asymmetrical $\mathrm{C}_{\alpha}=\mathrm{C}_{\beta}$ region did not develop 
a) Raman spectra depicting the SWNT $G$ and D bands

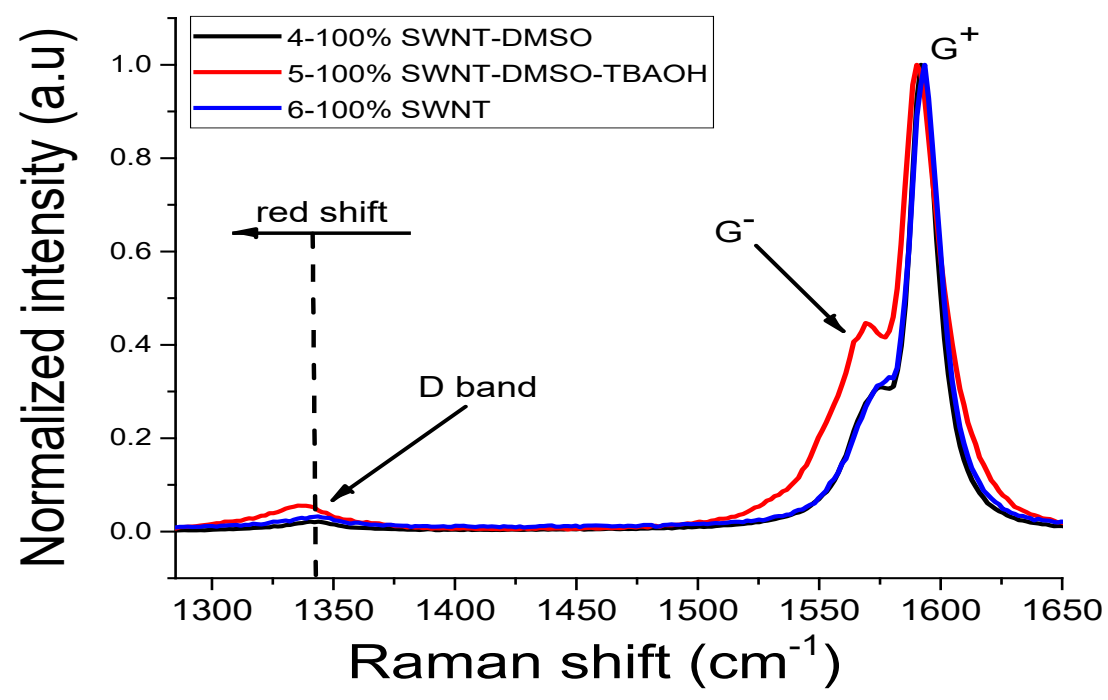

b) Raman spectra depicting SWNT 2D band

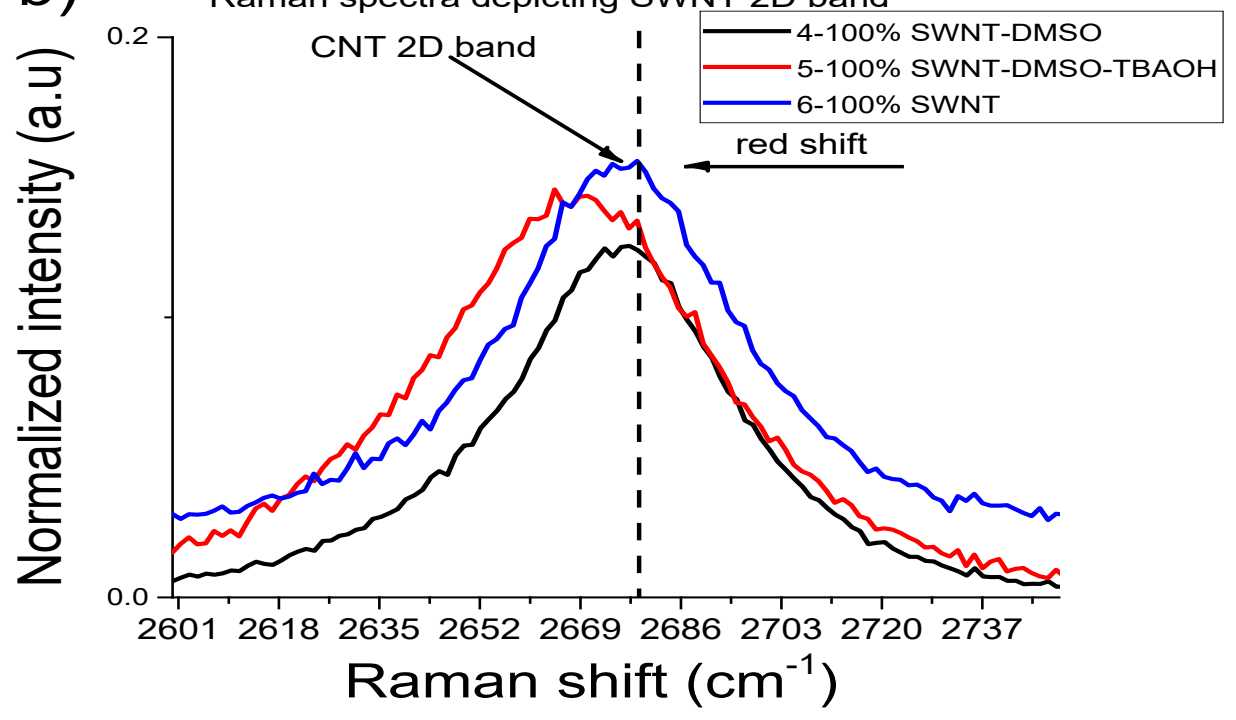

Figure 6.6.3 depicting the SWNT based controls in which the (a) depicts the D band's red shift as well as the $G$ to $D$ ratio of carbon nanotubes and (b) depicts the $2 D$ band and the corresponding red shift at the region.

Table 6.6.1 depicting the Raman shifts for the 1-6 films (fundamental controls), the SWNT-PEDOT:PSS-conc(DMSO and TBAOH treated) and SWNT-PEDOT:PSScont (DMSO treated) films as well as the XPS BE for PEDOT's Sulphur $2 \mathrm{p}_{3 / 2}$ region

\begin{tabular}{|l|l|l|}
\hline Film & $\begin{array}{l}\text { Raman shift at the specified } \\
\text { region }(\mathrm{cm}-1)\end{array}$ & $\begin{array}{l}\text { XPS binding energy } \\
(\mathrm{BE}) \text { for the PEDOT } \\
\text { Sulphur 2 } \mathrm{p}_{3 / 2} \text { peak }(\mathrm{eV})\end{array}$ \\
\hline
\end{tabular}




\begin{tabular}{|c|c|c|c|}
\hline & $\begin{array}{l}\mathrm{C}_{\alpha}=\mathrm{C}_{\beta} \text { benzoid to } \\
\text { quinoid }\end{array}$ & $\begin{array}{l}\mathrm{G}^{+} \\
\text {band }\end{array}$ & \\
\hline 1-pristine PEDOT:PSS & 1435 & & 163.90 \\
\hline $\begin{array}{l}\text { 2-pristine PEDOT:PSS- } \\
\text { DMSO }\end{array}$ & 1430 & & 163.84 \\
\hline $\begin{array}{l}\text { 3-pristine PEDOT:PSS- } \\
\text { DMSO-TBAOH }\end{array}$ & 1425 & & \\
\hline $4-100 \%$ SWNT-DMSO & & 1592 & \\
\hline $\begin{array}{ll}5-100 \% & \text { SWNT-DMSO- } \\
\text { TBAOH } & \end{array}$ & & 1590 & \\
\hline $6-100 \%$ SWNT & & 1593 & \\
\hline $\begin{array}{l}5 \% \text { SWNT-PEDOT:PSS- } \\
\text { conc }\end{array}$ & 1425 & 1589.8 & 163.60 \\
\hline $\begin{array}{l}10 \% \quad \text { SWNT- } \\
\text { PEDOT:PSS-conc }\end{array}$ & 1425 & 1589.8 & 163.64 \\
\hline $\begin{array}{l}20 \% \quad \text { SWNT- } \\
\text { PEDOT:PSS-conc }\end{array}$ & 1425 & 1589.8 & 163.57 \\
\hline $\begin{array}{l}40 \% \quad \text { SWNT- } \\
\text { PEDOT:PSS-conc }\end{array}$ & 1423 & 1589.8 & 163.59 \\
\hline $\begin{array}{l}80 \% \quad \text { SWNT- } \\
\text { PEDOT:PSS-conc }\end{array}$ & 1425 & 1589.8 & 163.43 \\
\hline $\begin{array}{l}\text { 5\% SWNT-PEDOT:PSS- } \\
\text { cont }\end{array}$ & 1431 & 1589.8 & \\
\hline $\begin{array}{l}10 \% \quad \text { SWNT- } \\
\text { PEDOT:PSS-cont }\end{array}$ & 1433 & 1589.2 & \\
\hline $\begin{array}{l}20 \% \quad \text { SWNT- } \\
\text { PEDOT:PSS-cont }\end{array}$ & 1430 & 1589.8 & \\
\hline $\begin{array}{l}40 \% \quad \text { SWNT- } \\
\text { PEDOT:PSS-cont }\end{array}$ & 1425 & 1589.2 & \\
\hline $\begin{array}{l}80 \% \quad \text { SWNT- } \\
\text { PEDOT:PSS-cont }\end{array}$ & 1431 & 1589.8 & \\
\hline
\end{tabular}



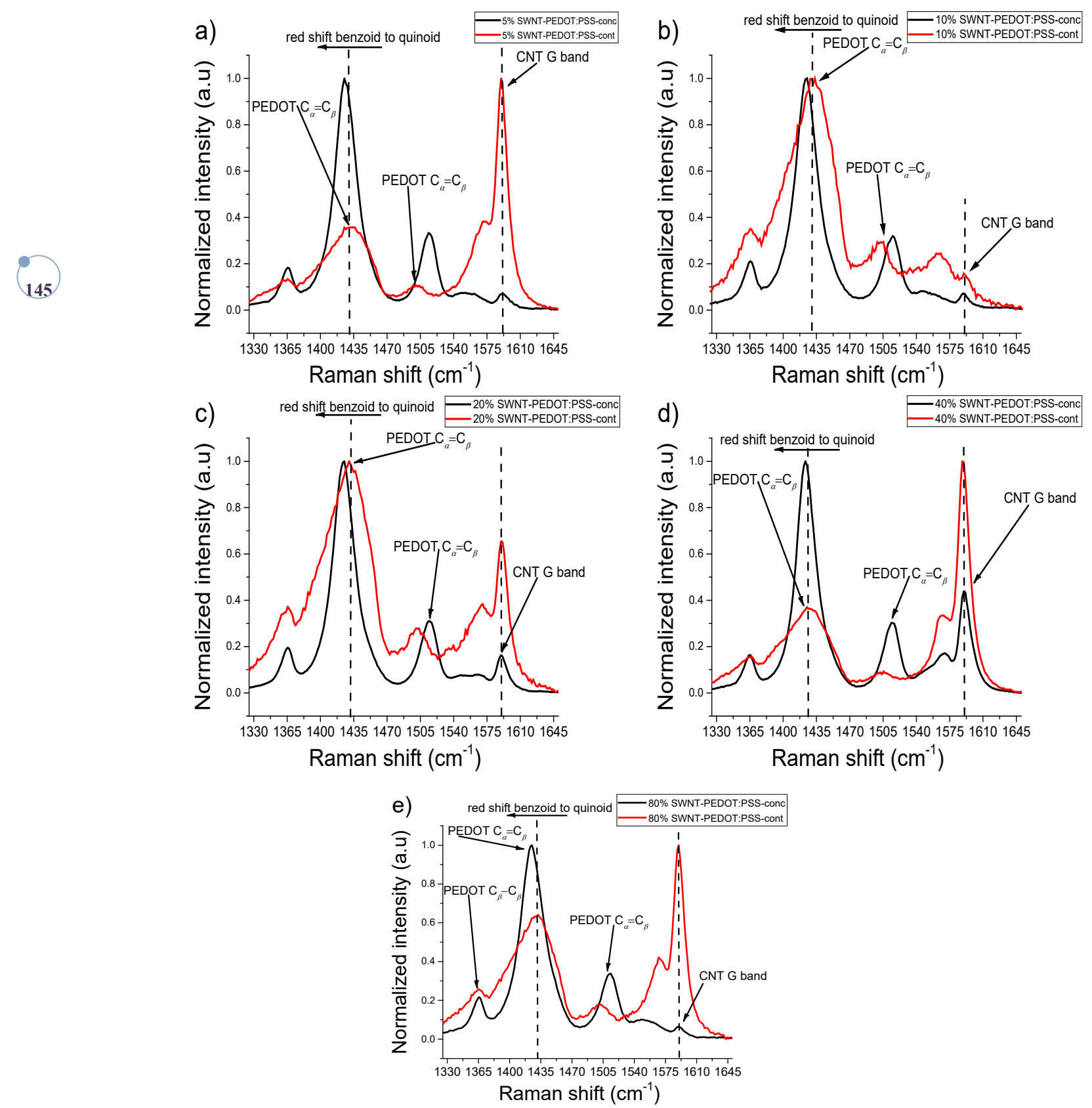

Figure 6.6.4 Depicts the overlaid Raman spectra for the for the SWNT-PEDOT:PSSconc and SWNT-PEDOT:PSS-cont films, (named within each overlaid spectra as appropriate). 


\subsection{Conclusion}

n-type SWNTs were synthesized by an extremely fast solvo thermal treatment method utilizing tetrabutyle ammonium hydroxide, (TBAOH) at $120^{\circ} \mathrm{C}$ for 40 seconds. It was also realized a method utilizing a concentration of $20 \% \mathrm{v} / \mathrm{v}$ SWNT, and $5 \% \mathrm{v} / \mathrm{v}$ DMSO to PEDOT:PSS with the TBAOH treatment at the temperature and time that an n-type SWNT-PEDOT:PSS composites can be synthesized. The n-type characteristics in the SWNT films were shown to be due to electron injection into the SWNT, as evidenced by the red shift at the G band. It was also realized that the SWNT-PEDOT:PSS-conc films that became n-type was due to two factors. First, the presence and reduction of SWNT particles via TBAOH as well as reduction of the p-type bipolaron carriers to at least polarons. The extent of the importance of the concentration of SWNT in the films determining the n-type characteristics is most probably due to the necessity of SWNT's n-type characteristics outweighing the p-type carriers in the thiophenes. The findings of this study will undoubtably influence more research into n-type PEDOT:PSS studies. Further studies looking at UV-Vis-NIR as well as full XPS characterisation of the films will be required to make more in depth analysis including measuring changes in the Fermi level in the n-type films. More studies to improve the Power factor would be useful as well as making module with p-and n-type PEDOT:PSS. 


\section{Chapter 7 Towards decoupled Seebeck coefficient from electrical conductivity}

\subsection{Abstract}

A ternary SWNT-PEDOT:PSS-EMIM:TFSI composite was synthesized exhibiting a high-Power factor of $236 \mu \mathrm{W} \mathrm{m}{ }^{-1} \mathrm{~K}^{-2}$ at $140{ }^{\circ} \mathrm{C}$. Studies revealed this was achieved due to the simultaneously improved high electrical conductivity and Seebeck coefficient of $3665 \mathrm{~S} \mathrm{~cm}^{-1}$ and $22 \mu \mathrm{V} \mathrm{K}^{-1}$ respectively. XPS studies showed that films post treated with ethylene glycol or the guanidinium iodide solution (GuI) caused significant phase separation between PEDOT and PSS and thereby resulted in selective removal of PSS. It was also evidenced a new peak towards lower binding energy indicating presence of Sulphur in a lower oxidation state $\left(\mathrm{S}^{2-}\right)\left(\mathrm{SH}_{2}\right)$. It was also revealed via UV-Vis-NIR and XPS spectra that a redox reaction in the thin film occurred whereby some PEDOT ${ }^{2+}$ molecules were reduced to $\mathrm{PEDOT}^{+}$due to the $\mathrm{I}^{-}$ thus partially explaining the increased Seebeck coefficient. It is argued that the guanidinium ion acted on the films by disrupting the ionic interaction between $\mathrm{PEDOT}^{+}$and $\mathrm{PSS}^{-}$and caused significant phase separation and acting as a catalyst for desulphonation of the sulfonate group pertaining to PSS . Finally, it is thought that the reason for the simultaneous improvement in both electrical conductivity and Seebeck coefficient is possibly due to the guanidinium ions that were shown to be adsorbed on the films may be involved in cation $-\pi$ intereactions thereby leading to overall increased bulk carrier concentration despite losing some thiophene carriers (bipolarons). 


\subsection{Introduction}

The pristine PEDOT:PSS films have been shown to have electrical conductivity of $<10 \mathrm{~S} \mathrm{~cm}^{-1}$ and Seebeck coefficient of $<15 \mu \mathrm{V} \mathrm{K} \mathrm{K}^{-1}$ at room temperature. ${ }^{188,337}$ Hence, for organic and hybrid thermoelectric generators (OHTEGs) to be effective in practical application they must exhibit a high thermoelectric efficiency which is described by the dimensionless Figure of merit, ZT. $^{89,155,220,222,337,402,413}$

$$
Z T=\frac{S^{2} \sigma \mathrm{T}}{\kappa}
$$

Whereby $\sigma$ is the electrical conductivity, $\mathrm{S}$ is the Seebeck coefficient, $\mathrm{T}$ is the absolute temperature, and $\kappa$ is the thermal conductivity. ${ }^{17,148,155,219,227} \kappa$ can be controlled by using a nanostructured phonon scattering polymer matrix. Although single walled carbon nanotubes (SWNT) exhibit high $\kappa$ It's been shown even at high carbon nanotube loading $(80 \% \mathrm{w} / \mathrm{w}) \kappa$ remains relatively low $\left(0.2-0.6 \mathrm{~W} \mathrm{~m}^{-1} \mathrm{~K}^{-1}\right)$ in Poly $(3,4-$ ethylenedioxythiophene)poly(4-styrenesulfonate) PEDOT:PSS-SWNT composites. ${ }^{67,197,207}$ The Seebeck coefficient can be further enunciated by applying it to Boltzmann transport theory as expressed in equation 2. Equation 3 denotes the relationship between charge carrier mobility and concentration to electrical conductivity.

$$
S=\frac{8 \pi^{2} k_{B}^{2}}{3 e h^{2}} m * T\left(\frac{\pi}{3 n}\right)^{\frac{2}{3}}
$$




$$
\sigma=n e \mu
$$

As contained in equation (2) and (3), $k_{B}$ is the Boltzmann constant, e conforms to the carrier charge, $\mathrm{h}$ corresponds to the Planck's constant, meanwhile, $m$ is the effective mass of the charge carrier, $n$ denotes the carrier concentration, and $\mu$ the carrier mobility. ${ }^{75,76}$ As evident, the Seebeck coefficient is inversely proportional to carrier concentration of which is positively proportional to electrical conductivity. Therefore, to improve both $\mathrm{S}$ and $\sigma$ to maximise power factor an improved carrier mobility would be required to offset the reduced carrier concentration.

$$
P F=S^{2} \sigma
$$

The PF of PEDOT:PSS nanocomposites can be improved via several strategies. In a study by Lee et al an ethylene glycol post treatment on a PEDOT:PSS/CNT composite was employed to yield a high PF of $151 \mu \mathrm{W} \mathrm{m} \mathrm{m}^{-1} \mathrm{~K}^{-2}$. 145 The performance of the composite was derived from the intrinsically high conductivity of the carbon nanotube and the morphological change induced by selective removal of PSS due to the ethylene glycol treatment which further improved the electrical conductivity with slight but not significant reduction in the Seebeck coefficient for the $20 \%$ CNT to PEDOT:PSS composite. ${ }^{145}$

Other methods involve multiple steps with variations of internal solvent treatment followed by post (synthesis of nanocomposite) treatment with reducing solutions to optimise PF. ${ }^{149,219,227,229,275}$ A recent study by Liu et al achieved a record high PF by incorporating SWNTs within PEDOT:PSS. As the SWNT were added to the polymer matrix up to $50 \%$ by weight, both (S) and ( $\sigma$ ) increased and a power factor of $190 \mu \mathrm{W}$ $\mathrm{m}^{-1} \mathrm{~K}^{-2}$ was obtained. ${ }^{71}$ There was a further increase in the electrical conductivity in 
different set of films prepared with DMSO treatment inducing a morphological change to improve carrier mobility. The Seebeck coefficient although increased with carbon nanotube content was not affected by DMSO treatment. This led to a PF of $270 \mu \mathrm{W}$ $\mathrm{m}^{-1} \mathrm{~K}^{-2}$. The optimised film with DMSO treatment and a further $0.1 \mathrm{M} \mathrm{NaOH}$ post treatment led to a high $\mathrm{PF}$ of $526 \mu \mathrm{W} \mathrm{m} \mathrm{m}^{-1} \mathrm{~K}^{-2} \cdot{ }^{71}$ It is known that reducing PEDOT molecules leads to improved Seebeck coefficient as shown in several studies. ${ }^{139,209,217,368}$ It's however acknowledged in literature that reducing agents usually have negative impact on the electrical conductivity. ${ }^{350,409}$

In other work a triple post treatment was conducted on PEDOT:PSS to yield a high PF of $141 \mu \mathrm{W} \mathrm{m}{ }^{-1} \mathrm{~K}^{-2}$ at room temperature. ${ }^{276}$ This was due to the fine tuning of oxidation level through reducing agent $\left(\mathrm{NaBH}_{4}\right)$ which improved the Seebeck coefficient to 28 $\mu \mathrm{V} \mathrm{K}^{-1}$ and treatments with Formamide $\left(\mathrm{CH}_{3} \mathrm{NO}\right)$ and sulfuric acid $\left(\mathrm{H}_{2} \mathrm{SO}_{4}\right)$ which improved electrical conductivity to $1786 \mathrm{~S} \mathrm{~cm}^{-1} .^{276}$

There is effectively a trade-off whereby the reduced carrier concentration leads to reduced electrical conductivity (please refer to equations (2, and 3). Moreover, it's been shown in some studies that exposure to air causes reduced PEDOT films to rapidly oxidise thus though time a reduction of the Seebeck coefficient is observed. ${ }^{139}$ A recent study however by Kee et al. ${ }^{218}$ has shown that treatments with ionic liquids can improve stability of de-doped films under ambient air and light via ionic interaction between ionic liquid counter ions and reduced PEDOT molecules. ${ }^{218}$

Moreover, these ionic liquid composites have been shown to significantly improve $(\sigma)$ without reducing $(\mathrm{S})$ and in some cases improve both the $(\mathrm{S})$ from $(\sigma)$ via post treatment. ${ }^{356,357,382,414,415}$ Significantly higher electrical conductivity has been shown by controlling molecular ordering in PEDOT:PSS chains by using 1-ethyl-3methylimidazolium tetracyanoborate (EMIM:TCB), achieving an electrical conductivity of $2103 \mathrm{~S} \mathrm{~cm}^{-1} .^{217}$ PEDOT:PSS-ionic liquid composites have also exhibited resistance to mechanical deformation while maintaining high performance thereby making them candidates for applications in flexible thermoelectrics. ${ }^{218,368}$

Ionic liquids treatments utilizing lithium, (trifluoromethane) sulfonimideimide $\left(\mathrm{LI}^{+} \mathrm{TFSI}^{-}\right)$on PEDOT:PSS at room temperature have improved $(\sigma)$ up to $1000 \mathrm{~S} \mathrm{~cm}^{-}$ ${ }^{1}$ without affecting $\mathrm{S}$, yielding a high $\mathrm{PF}$ of $75 \mu \mathrm{W} \mathrm{m} \mathrm{m}^{-1} \mathrm{~K}^{-2}$ due to an optimised concentration 60 wt.\% LI $^{+}$TFSI $^{-}$to PEDOT:PSS). ${ }^{416}$ Although TFSI $^{-}$has a lone pair of 
electrons on the nitrogen it may be stable to the extent proving difficult for an electron on the lone pair to be donated to the thiophene backbone and cause a reduction of the bipolarons. As observed in the UV-Vis-NIR spectra of the films treated with EMIM:TFSI in chapter 5 there were no signs of bipolaron reduction. In a study by Liu et al. ${ }^{416}$ it was also shown that the TFSI didn't cause a reduction of bipolarons as evidenced by the lack of improvement in the Seebeck coefficient. In a recent study it was discovered that the Seebeck coefficient and electrical conductivity could be improved by an ionic liquid treatment on PEDOT:PSS with a component that can reduce the coulombic interaction between PEDOT and PSS thus selectively remove PSS meanwhile another component reduces thiophene molecules to improve S. ${ }^{359}$

Guanidinium iodide is a salt . In this study it was dissolved in ethylene glycol. It has not been reported as a dopant in PEDOT:PSS thermoelectrics however in literature its effects as a protein denaturant is known whereby it disrupts the hydrogen bonding between base pairs. ${ }^{417,418}$ Guanidinium iodide and other guanidinium derivatives have been studied in cases for improving stability of perovskite solar cells as seen in literature literature. ${ }^{419-423}$ In a recent study by Hong et al, ${ }^{424}$ it was shown that guanidinium iodide treatment on perovskite film surface improved both the moisture stability as well as efficiency from $19 \%$ to $20 \%$. In this study guanidinium Iodide is used as the treatment agent to simultaneously improve $S$ and $(\sigma)$ due to reducing properties of the conjugate base $\mathrm{I}^{-}$and the ability of the guanidinium Ion to disrupt the coulombic interaction between PSS and PEDOT thereby leading to selective PSS removal from film. ${ }^{425-427}$ 


\subsection{Objective and overview}

\section{Key definitions}

Primary doping: the definition used to describe the procedure employed to synthesise PEDOT:PSS from EDOT and $\mathrm{PSS}^{-}$with $\mathrm{FeCl}_{3}$ in which the product PEDOT:PSS (PEDOT is oxidised and majority of charge it's carriers are (bipolaronic) $\mathrm{PEDOT}^{2+} \cdot 428$

Secondary doping: used by MacDiarmid and Epstein to state an inert or otherwise doping mechanism that does not necessarily change the oxidation state of the bulk material. Some examples of such doping include majority of the solvent treatment observed in literature as well as some ionic liquid treatments such as EMIM:TFSI on PEDOT:PSS. ${ }^{196,429,430}$

Dedoping; used to describe reducing some of the thiophene rings. Some examples include using a reducing agent treatment on PEDOT:PSS. ${ }^{127,128,195}$

The $2.5 \%$ EMIM:TFSI treatment as shown in chapter 5 significantly increased the electrical conductivity of PEDOT:PSS from $3 \mathrm{~S} \mathrm{~cm}^{-1}$ to $1439 \mathrm{~S} \mathrm{~cm}^{-1}$ at $40{ }^{\circ} \mathrm{C}$. When post treated with the $1 \mathrm{w} / \mathrm{v} \% \mathrm{NaBH}_{4}$-DMSO reducing agent there was a trade-off between the now reduced electrical conductivity and the significantly improved Seebeck coefficient leading to a power factor of $33 \mu \mathrm{W} \mathrm{m} \mathrm{m}^{-1} \mathrm{~K}^{-2}$ at the same temperature. However, to transcend this trade-off it is pertinent that redox of thiophenes is controlled as to yield a desired carrier concentration whereby the Seebeck coefficient improves significantly without significant loss in the electrical conductivity. As stated in the paper published from the results in chapter 5, it was discovered that a concentration in the range of $1-2.5 \mathrm{v} / \mathrm{v} \%$ EMIM:TFSI to PEDOT:PSS may be used to improve the electrical conductivity without negatively affecting the Seebeck coefficient thereby leading to PFs that are significantly higher than the pristine film. ${ }^{2}$ Attempts to further enhance the PF was realized however it was discovered that the reducing agent $\left(1 \mathrm{w} / \mathrm{v} \% \mathrm{NaBH}_{4}\right)$ invariably reduced the electrical conductivity when it was utilized to improve the Seebeck coefficient. The following line of logic was utilized to develop the procedure expressed in chapter 7.4. 
1. EMIM:TFSI can significantly improve the electrical conductivity however has no effect on the Seebeck coefficient. $^{338}$

2. EMIM:TFSI has been shown to improve the electrical conductivity of CNTs in literature. ${ }^{431}$

3. Dedoping the PEDOT:PSS via reducing agent such as $\mathrm{NaBH}_{4}$ can increase the Seebeck coefficient however a reduction of the electrical conductivity is observed. ${ }^{408}$

4. Treating SWNTs with reducing agents have been shown in literature to improve the electrical conductivity. ${ }^{295,432,433}$

5. Therefore, if utilizing a dedopant that which can donate electrons to the SWNT-PEDOT:PSS composite to improve the Seebeck coefficient meanwhile itself being adsorbed onto the film to maintain overall surface charge carriers, then perhaps the cost on the electrical conductivity would not be observed.

6. In this chapter it is reported several novel composites as well as a solvothermal treatment method for dedoping thiophene rings of PEDOT:PSS using a protein denaturant guanidinium iodide (GuI) to induce desired oxidation state. Moreover, presented in this study is the synthesis of a novel PEDOT:PSS-EMIM:TFSI-SWNT composite and a subsequent post treatment with GuI solution to significantly improve the Power factor without exhibiting a trade-off between Seebeck coefficient and electrical conductivity.

7. 


\subsection{Materials and methods}

Please refer to chapter 3.2. for a list of all materials used.

\subsubsection{Synthesis of guanidinium iodide solution (GuI solution)}

$60 \mathrm{mg}$ of guanidinium iodide solid was placed in a $7 \mathrm{~mL}$ glass vial and mixed with $1000 \mu \mathrm{L}$ ethylene glycol solution and stored in a cool dry place. (After an hour complete dissolution was observed).

\subsubsection{Synthesis of PEDOT:PSS-EMIM:TFSI composites}

$3000 \mu \mathrm{L}$ of PH1000 PEDOT:PSS was placed in a $7 \mathrm{~mL}$ glass vial in the presence of $65 \mu \mathrm{L}$ of EMIM:TFSI which was mixed using a vortex mechanical mixer in ultra-sonic water bath for 30 minutes.

\subsubsection{Synthesis of SWNT-PEDOT:PSS composites}

$3000 \mu \mathrm{L}$, of PH1000 PEDOT:PSS solution was placed in a $7 \mathrm{~mL}$ glass vial and mixed with $1200 \mu \mathrm{L}$ of the $0.5 \%$ SWNT solution (forming a $40 \% \mathrm{v} / \mathrm{v}$ solution mixture) using vortex mechanical mixer and a subsequent (30-minute ultrasonic water bath) at room temperature.

\subsubsection{Synthesis of SWNT-PEDOT:PSS-EMIM:TFSI composites}

$3000 \mu \mathrm{L}$ of PH1000 PEDOT:PSS was mixed with $65 \mu \mathrm{L}$ of EMIM:TFSI and a subsequent $1200 \mu \mathrm{L}$ of $0.5 \%$ SWNT in a $7 \mathrm{~mL}$ glass vial was mixed with the vortex mechanical mixer and subjected to ultrasound treatment for 30 minutes.

\subsubsection{Spin coating of thin film composites}

$1 \mathrm{~mm}$ thick plain nonconductive glass was cut into $2.2 \mathrm{~cm}$ by $2.2 \mathrm{~cm}$ pieces and washed in a Helmanax solution. The glass substrates were then rinsed with deionised water and subsequently rinsed with acetone and then isopropanol for at least 3 times. The glass was then dried in a stream of nitrogen gas. $256 \mu \mathrm{L}$ of the appropriate solutions were then pipetted onto a pre-cleaned glass substrate and spin-coated at $2000 \mathrm{rpm}$ with an acceleration of $2000 \mathrm{rpm} \mathrm{s}^{-1}$ for 30 seconds. The films were then annealed at $154 \mid \mathrm{P}$ a g e 
$120^{\circ} \mathrm{C}$ in air (fume hood) for 15 minutes resulting in films between $50-70 \mathrm{~nm}$ thick, as determined by Dektak surface profilometry.

\subsubsection{Post treatment of thin film composite with guanidinium iodide solution}

For each composite film at $160{ }^{\circ} \mathrm{C} 100 \mu \mathrm{L}$ of the as prepared GuI solution was pipetted on the surface of the film and allowed to react for 10 minutes, (it was observed that majority of solution had evaporated therefore another $100 \mu \mathrm{L}$ GuI solution was placed on the surface of the film at the same conditions. For the controls $(\mathrm{CX})$, a pristine ethylene glycol solution was used as the post treatment agent and the pristine PEDOT:PSS (FC1) remained without any post treatment.

\subsection{Results and discussion}

\subsubsection{Thermoelectric properties of fundamental control composites}

To determine the origin of the improved thermoelectric properties of the GuI treated films as well as other composites developed in this study several controls were conducted. There were two sets of controls. Figure 7.5.1 shows the thermoelectric properties of the $1^{\text {st }}$ set of controls (FCX where X refers to a number between 1 and 4 depicting systematic variations within the sample composition across a measured temperature range. As shown in Figure 7.5.1 (a) the electrical conductivity of the FC1 pristine PEDOT:PSS film is within values exhibited in literature. ${ }^{29,361,408}$ When the PEDOT:PSS film is composited with the SWNT, the electrical conductivity is not improved. This is most likely due to the SWNTs in this study being intrinsically poorly conductive. ${ }^{145}$ Across the temperature range measured, both the FC1 pristine PEDOT:PSS and FC2 SWNT-PEDOT:PSS films have low electrical conductivity that do not exceed $8 \mathrm{~S} \mathrm{~cm}^{-1}$. At $40{ }^{\circ} \mathrm{C}$ both films previously mentioned have an electrical conductivity of around $3.4 \mathrm{~S} \mathrm{~cm}^{-1}$. The FC3 PEDOT:PSS-EMIM:TFSI was made by a $2 \% \mathrm{v} / \mathrm{v}$ EMIM:TFSI to PEDOT:PSS solution. ${ }^{2}$ The question one may ask is if the $2.5 \%$

$\mathbf{1 5 5} \mid \mathrm{P}$ a g e 
EMIM:TFSI film as exhibited in the results from chapter 5 had superior electrical conductivity than the 2\% EMIM:TFSI treatment film then why was later treatment described utilized for this study? The reason becomes clear when conducting the preliminary experiments. It was observed that when creating the ternary (SWNTPEDOT:PSS:EMIM:TFSI) composites, that solution aggregation would occur on addition of either the ionic liquid or SWNT to the PEDOT:PSS-Y solution where $\mathrm{Y}$ is EMIM:TFSI or SWNT. Therefore, a compromise to utilize the $2 \% \mathrm{v} / \mathrm{v}$ EMIM:TFSI to PEDOT treatment was accepted. As shown within Figure 7.5.1 (a) the FC3 PEDOT:PSS-EMIM:TFSI film has a much higher electrical conductivity above $700 \mathrm{~S}$ $\mathrm{cm}^{-1}$ at $40{ }^{\circ} \mathrm{C}$ than the previous $\mathrm{FC} 1$ or FC2 films previously discussed. The $700 \mathrm{~S} \mathrm{~cm}^{-}$ ${ }^{1}$ mentioned is similar to published results for high performing ethylene glycol and DMSO treatments. ${ }^{337,372,434}$ The FC4 SWNT-PEDOT:PSS-EMIM:TFSI film had a significantly higher electrical conductivity than all films in Figure 7.5.1 (a) across the whole temperature range. At $40{ }^{\circ} \mathrm{C}$ the electrical conductivity for the film was $1540 \mathrm{~S}$ $\mathrm{cm}^{-1}$. As the temperature increased the electrical conductivity reduced slightly until a minimum of $1230 \mathrm{~S} \mathrm{~cm}^{-1}$ at $183^{\circ} \mathrm{C}$. Both the FC4 SWNT:PEDOT:PSS-EMIM:TFSI and the FC3 PEDOT:PSS-EMIM:TFSI film would be considered (non-thermally assisted hopping) films due to their lack of increased conductivity at higher temperatures. ${ }^{435-437}$ However, for the FC1 pristine PEDOT:PSS film and the FC2 SWNT-PEDOT:PSS film it's not clear how temperature affects charge carrier. This is because for the pristine PEDOT:PSS film there is a small albeit gradual increase in electrical conductivity from $3.4 \mathrm{~S} \mathrm{~cm}^{-1}$ at $40{ }^{\circ} \mathrm{C}$ to $5.4 \mathrm{~S} \mathrm{~cm}^{-1}$ at $180^{\circ} \mathrm{C}$. For the SWNTPEDOT:PSS there is an increase however there are also dips at various temperatures therefore a generalized pattern cannot confirm a thermally assisted hopping for the entire temperature range measured.

Figure 7.5.1 (b) depicts the Seebeck coefficient for the fundamental Controls (FCX) films across the temperature range $\left(40{ }^{\circ} \mathrm{C}\right.$ to $\left.<200{ }^{\circ} \mathrm{C}\right)$. All films exhibit p-type characteristics as expressed by the positive sign of the Seebeck coefficient. ${ }^{438,439}$ The pristine PEDOT:PSS film exhibits a Seebeck coefficient of $13 \mu \mathrm{V} \mathrm{K}^{-1}$ at $40{ }^{\circ} \mathrm{C}$ which slowly increases to $18 \mu \mathrm{V} \mathrm{K}^{-1}$ at $180{ }^{\circ} \mathrm{C}$ thereby showing a positive relationship between temperature and Seebeck coefficient. ${ }^{403,440,441}$ The FC3 PEDOT:PSSEMI:TFSI film's Seebeck coefficient shows a steady but gradual decrease from $14 \mu \mathrm{V}$ 
$\mathrm{K}^{-1}$ at $40{ }^{\circ} \mathrm{C}$ to $10 \mu \mathrm{V} \mathrm{K}^{-1}$ at $170{ }^{\circ} \mathrm{C}$ then a slight increase to $12 \mu \mathrm{V} \mathrm{K}^{-1}$ at $190{ }^{\circ} \mathrm{C}$. In conjunction with Figure 7.5.1 (a) for the FC3 PEDOT:PSS-EMIM:TFSI film's electrical conductivity and Seebeck coefficient, both show a negative correlation with temperature. The FC4 SWNT-PEDOT:PSS-EMIM:TFSI film showed a positive correlation between the Seebeck coefficient and temperature. The film with the highest Seebeck coefficient was the SWNT-PEDOT:PSS film with a Seebeck coefficient of $20 \mu \mathrm{V} \mathrm{K}^{-1}$ at $40^{\circ} \mathrm{C}$ which gradually increases to an optimum of $29 \mu \mathrm{V} \mathrm{K}^{-1}$ at $160{ }^{\circ} \mathrm{C}$.

As shown in Figure 7.5.1 (c) the film with the highest power factor was the FC4 SWNT-PEDOT:PSS-EMIM:TFSI in which at $40^{\circ} \mathrm{C}$ the power factor was $22 \mu \mathrm{W}$ $\mathrm{m}^{-1} \mathrm{~K}^{-2}$ gradually rising to $50 \mu \mathrm{W} \mathrm{m} \mathrm{m}^{-1} \mathrm{~K}^{-2}$ at $130^{\circ} \mathrm{C}$, then decreasing slightly and finally reaching a maximum of $57 \mu \mathrm{W} \mathrm{m} \mathrm{K}^{-1}$ at $180{ }^{\circ} \mathrm{C}$. The reason for the considerably higher power factor relative to all other films was primarily due to its increase in the Seebeck coefficient, $\left(11 \mu \mathrm{V} \mathrm{K}^{-1}\right.$ at $40{ }^{\circ} \mathrm{C}$ rising to $18 \mu \mathrm{V} \mathrm{K}^{-1}$ at $\left.130{ }^{\circ} \mathrm{C}\right)$; coupled with its extremely high electrical conductivity $\left(1430 \mathrm{~S} \mathrm{~cm}^{-1}\right.$ at $\left.130^{\circ} \mathrm{C}\right)$. Although the $\mathrm{FC} 2$ SWNT-PEDOT:PSS film had the most notable Seebeck coefficient, significantly higher than all other films in Figure 7.5.1 (b), its power factor was only slightly higher than pristine PEDOT:PSS due to its extremely low electrical conductivity. Therefore, because the FC3 PEDOT:PSS-EMIM:TFSI film had high electrical conductivity and a Seebeck coefficient not significantly lower than other films, its peak power factor was $15 \mu \mathrm{W} \mathrm{m}{ }^{-1} \mathrm{~K}^{-2}$ at $40{ }^{\circ} \mathrm{C}$ which slowly reduced across the temperature range following the same pattern as it is electrical conductivity (Figure 7.5.1 (a)). 
a)
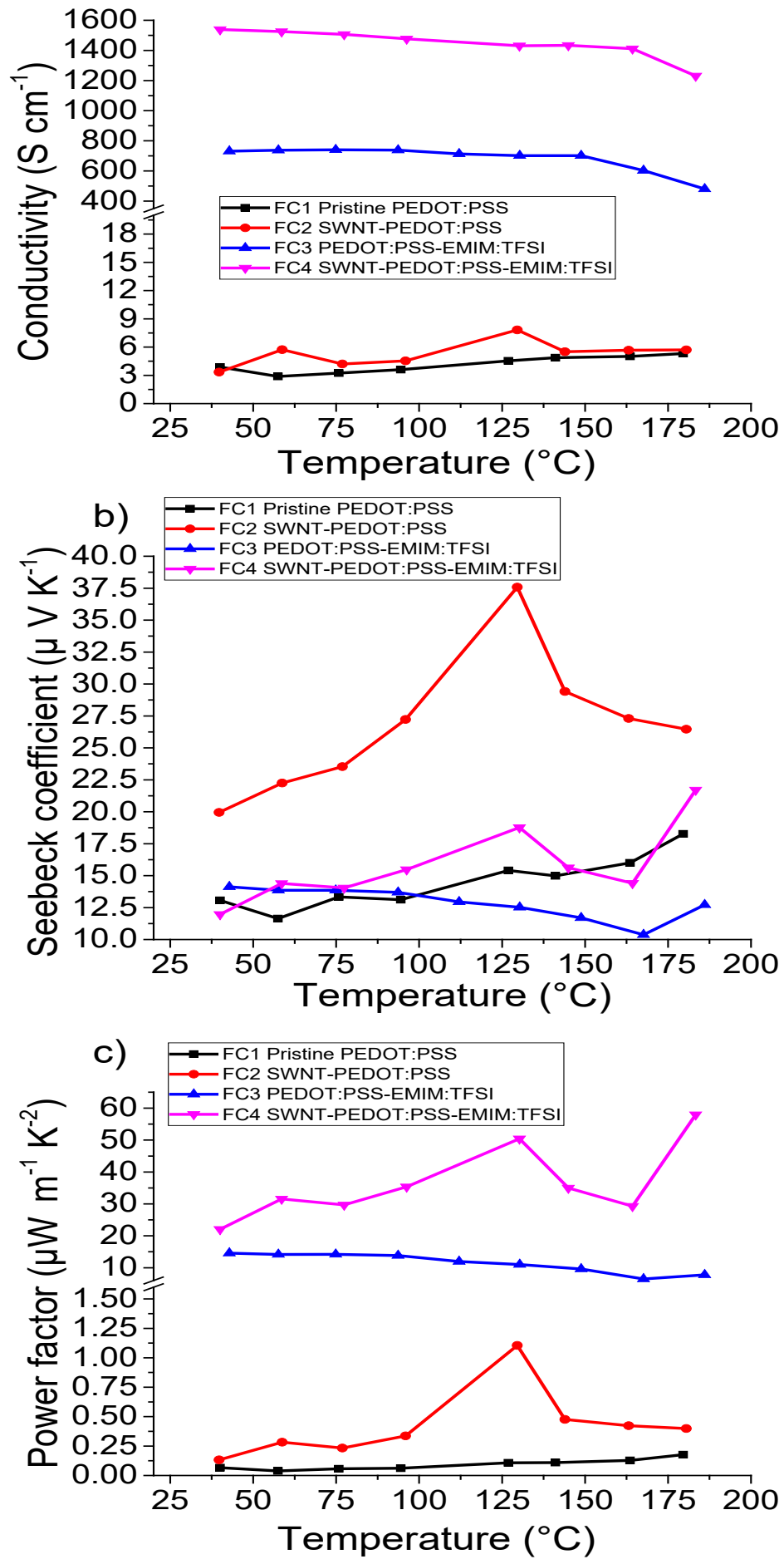

Figure 7.5.1, depicting the electrical conductivity (a) Seebeck coefficient (b) and Power factor (c) for the Fundamental Controls (FCX) films against a temperature range $\left(40^{\circ} \mathrm{C}-200^{\circ} \mathrm{C}\right)$. 


\subsubsection{Thermoelectric properties of guanidinium iodide treated films.}

Figure 7.5.2.1 shows the as measured electrical conductivity against an increasing temperature profile for the (CX, (a) and SX films (b), whereby X depicts a number between 1 and 4 in which it represents different sample composition within each set (C or S). The CX films were synthesized in the same manner except for the post treatment. The solvent used for the SX films was ethylene glycol. In other words, the CX films were post treated with ethylene glycol that did not contain GuI thus differentiating them from the SX films to function as controls. Figure 7.5.1 (a) shows the electrical conductivity against temperature for the FCX films (FC1-FC4). The FC1FC4 films were a novel class of materials synthesized in the same manner as the CX films except they were not post treated with ethylene glycol. The FCX films acted as a fundamental control set to deduce the effects, if any on the Seebeck coefficient and or electrical conductivity introduced to the films, with the ethylene glycol and guanidinium iodide post treatment.

Figure 7.5.2.1 (a) and (b) shows a general trend whereby for all films there is an inverse relationship between the electrical conductivity and the temperature it is measured at. Because the increase in temperature does not lead to an increased electrical conductivity, both the CX and SX films may be characterised as having a carrier transport propagated via (non-thermally assisted hopping), in some cases dubbed (thermally independent hopping) to describe transport not significantly improved by temperature as discussed in chapter 1.4. ${ }^{99,132,220,408,442}$ The $\mathrm{C} 1$ pristine PEDOT:PSS-EG, C2 SWNT-PEDOT:PSS-EG, and C3 PEDOT:PSS-EMIM:TFSI$\mathrm{EG}$, show a gradual decrease in electrical conductivity until around $130{ }^{\circ} \mathrm{C}$ where the decrease occurs more rapidly. For the C4 SWNT-PEDOT:PSS-EMIM:TFSI-EG film there is a gradual decrease in conductivity until a slightly higher temperature of 160 ${ }^{\circ} \mathrm{C}$ where the conductivity deteriorates more rapidly. For the (SX) films, all samples including the S4 SWNT-PEDOT:PSS-EMIM:TFSI-GuI film exhibit the rapid decrease in conductivity at the same point $\left(130^{\circ} \mathrm{C}\right)$. The relevance of the temperature the films show drastic decrease in conductivity may be pertinent when making a $159 \mid \mathrm{P}$ a g e 
functional device to determine thermal operating windows. ${ }^{443-445}$ Further studies to see if films can withstand multiple temperature cycles would be needed in future studies. The general trend is that the films with higher electrical conductivity have a more rapid decrease within the temperature range. The $\mathrm{CX}$ film with the highest electrical conductivity was the C4 SWNT-PEDOT:PSS-EMIM:TFSI-EG film in which at $40^{\circ} \mathrm{C}$ it was $2239 \mathrm{~S} \mathrm{~cm}^{-1}$.

Compared to one of the most conductive ionic liquid treatments on PEDOT:PSS using 1-ethyl-3-methylimidazolium tetracyanoborate (EMIM:TCB), (2103 $\left.\mathrm{S} \mathrm{cm}^{-1}\right)$, the $\mathrm{C} 4$ film was more conductive. ${ }^{217}$ When compared to some of the highest conductive PEDOT:PSS films using $\mathrm{H}_{2} \mathrm{SO}_{4}$ treatment resulting in $2673 \mathrm{~S} \mathrm{~cm}^{-}$ 1319,446 and record high $4600 \mathrm{~S} \mathrm{~cm}^{-1125,195,446}$ however the $\mathrm{C} 4$ film is not as conductive. When compared to its equivalent without the ethylene glycol treatment the (F4 SWNTPEDOT:PSS-EMIM:TFSI) film with conductivity of $1540 \mathrm{~S} \mathrm{~cm}^{-1}$ at the same temperature it is clear that the C4 SWNT-PEDOT:PSS-EMIM:TFSI-EG film is significantly more conductive. With a conductivity difference of around $699 \mathrm{~S} \mathrm{~cm}^{-1}$ it is evident that the ethylene glycol treatment positively affects the electrical conductivity of the SWNT-PEDOT:PSS-EMIM:TFSI composite. A Paper by Lee et $a l .{ }^{145}$ Showed that ethylene glycol when treated on a PEDOT:PSS/CNT composite improved the electrical conductivity from $<10 \mathrm{~S} \mathrm{~cm}^{-1}$ to slightly $<800 \mathrm{~S} \mathrm{~cm}^{-1}$. It was observed however that ethylene glycol treatment reduced the $\mathrm{S}$ of the composites. Figure 7.5.2.2 shows the Seebeck coefficient against a temperature profile for the $\mathrm{CX}$ (a) and SX (b) films. The Seebeck coefficient of the S4 SWNT-PEDOT:PSSEMIM:TFSI-GuI film is higher than both the $\mathrm{C} 4$ and FC4 equivalent. This is expected since it was discovered that the GuI treatment improved the Seebeck coefficient of the films. However, unlike the paper that found slight decrease in the Seebeck coefficient for ethylene glycol treatment on PEDOT:PSS/CNT films. ${ }^{145}$ in this case there is no net significant difference between the Seebeck coefficient of the C4 and FC4 SWNTPEDOT:PSS-EMIM:TFSI films as shown by Figure 7.5.1 (b) and 7.5.2.2 (b).

There is a clear pattern observed between the FCX, CX, and SX films' electrical conductivity. When comparing each composite to its molecular equivalent, the CX version is more conductive than its FCX form, meanwhile the SX form is more so conductive. For example, the FC3 PEDOT:PSS-EMIM:TFSI film's conductivity at 
$40^{\circ} \mathrm{C}$ is $731 \mathrm{~S} \mathrm{~cm}^{-1}$ meanwhile for the $\mathrm{C} 3$ film it is $880 \mathrm{~S} \mathrm{~cm}^{-1}$, and $\mathrm{S} 3\left(1146 \mathrm{~S} \mathrm{~cm}^{-1}\right)$ at the same temperature. This hierarchy in conductivity gives evidence that the ethylene glycol plays a role in improving the electrical conductivity when treated to all films. It is also evident that the GuI plays a significant role in improving the electrical conductivity. It appears that the films with SWNT composition benefits from the greatest enhancement in electrical conductivity with regards to EG treatment than the films with EMIM:TFSI, and finally the pristine PEDOT:PSS based films. The SWNT used in the study were poorly conducting. The increase in conductivity from the FC2 to $\mathrm{C} 2$ films after the addition of ethylene glycol significantly improved the electrical conductivity however from all films in the study the SWNT-PEDOT:PSS-X films were the lowest conductivity films. For the FC2 SWNT-PEDOT:PSS film its electrical conductivity was similar to $\mathrm{FC} 1$ pristine PEDOT:PSS at $3 \mathrm{~S} \mathrm{~cm}^{-1}$ at $40{ }^{\circ} \mathrm{C}$, in which after the ethylene glycol treatment it rose to $350 \mathrm{~S} \mathrm{~cm}^{-1}$. After the GuI treatment as shown for the S2 SWNT-PEDOT:PSS-GuI film it improved by a further $38 \mathrm{~S} \mathrm{~cm}^{-1}\left(388 \mathrm{~S} \mathrm{~cm}^{-1}\right)$. The increase was not significant and could have been due to fluctuations however it did follow the same trend as other films. When analysing, the film with the greatest enhancement it is evident from Figure 7.5.1 (a), and 7.5.2.1 (a) and (b) that the FC4, C4, and S4 (SWNT-PEDOT:PSS-EMIM:TFSI-X) films showed the greatest sensitivity to the treatments via significant electrical conductivity improvements. From FC4 at $40{ }^{\circ} \mathrm{C}\left(1540 \mathrm{~S} \mathrm{~cm}^{-1}\right)$ to the $\mathrm{C} 4\left(2240 \mathrm{~S} \mathrm{~cm}^{-1}\right)$ to the final treatment with GuI, leading to an optimised $\left(3665 \mathrm{~S} \mathrm{~cm}^{-1}\right)$. When compared to one of the best conductive PEDOT:PSS films with 74\% CNT loading film post treated with DMSO solvent ( $3800 \mathrm{~S} \mathrm{~cm}^{-1}$ ), the $\mathrm{S} 4$ film is slightly lower. ${ }^{447}$ However it's off note that the loading of CNT in this study was considerably lower at $40 \%$. From analysis of all samples, it is evident that the ethylene glycol treatment is involved in the initial improvement of electrical conductivity, in which films with EMIM:TFSI treatment are affected more strongly. It is also evident that the effect of ethylene glycol favours the conductivity of SWNT treated films to also improve as seen in literature. ${ }^{145}$ Finally, it's clear that there is a synergistic effect whereby the GuI treatment also partakes in improving the electrical conductivity as all films treated with the GuI solution show further improvements on conductivity as evidenced by the difference in conductivity 
between each succession of the treatments on the FC4, C4 and S4. Finding the mechanisms by which the improvement occurs will be explored in chapter 7.5.3 and 7.6.3.

As exhibited by Figure 7.5.2.2 all the CX and SX films exhibit p-type semiconductor behaviour as depicted by the positive sign of the Seebeck coefficient. ${ }^{448-450}$ At $40{ }^{\circ} \mathrm{C}$ as well as across majority of the temperature profile none of the CX films have a higher Seebeck coefficient than the SX films. This trend solidifies that the GuI treatment improves the Seebeck coefficient of PEDOT:PSS and its composites. From Figure 7.5.2.2 (a) when analysing the CX films, there is little variability between the Seebeck coefficient of the films especially at the lower temperatures. Compared to the FCX films each CX equivalent appears to have similarly low Seebeck coefficient at $40{ }^{\circ} \mathrm{C}$ which are comparable to that of the undoped film (FC1 pristine PEDOT:PSS at around $13 \mu \mathrm{V} \mathrm{K}^{-1}+/-1.5$ except for the FC2 SWNTPEDOT:PSS film. 


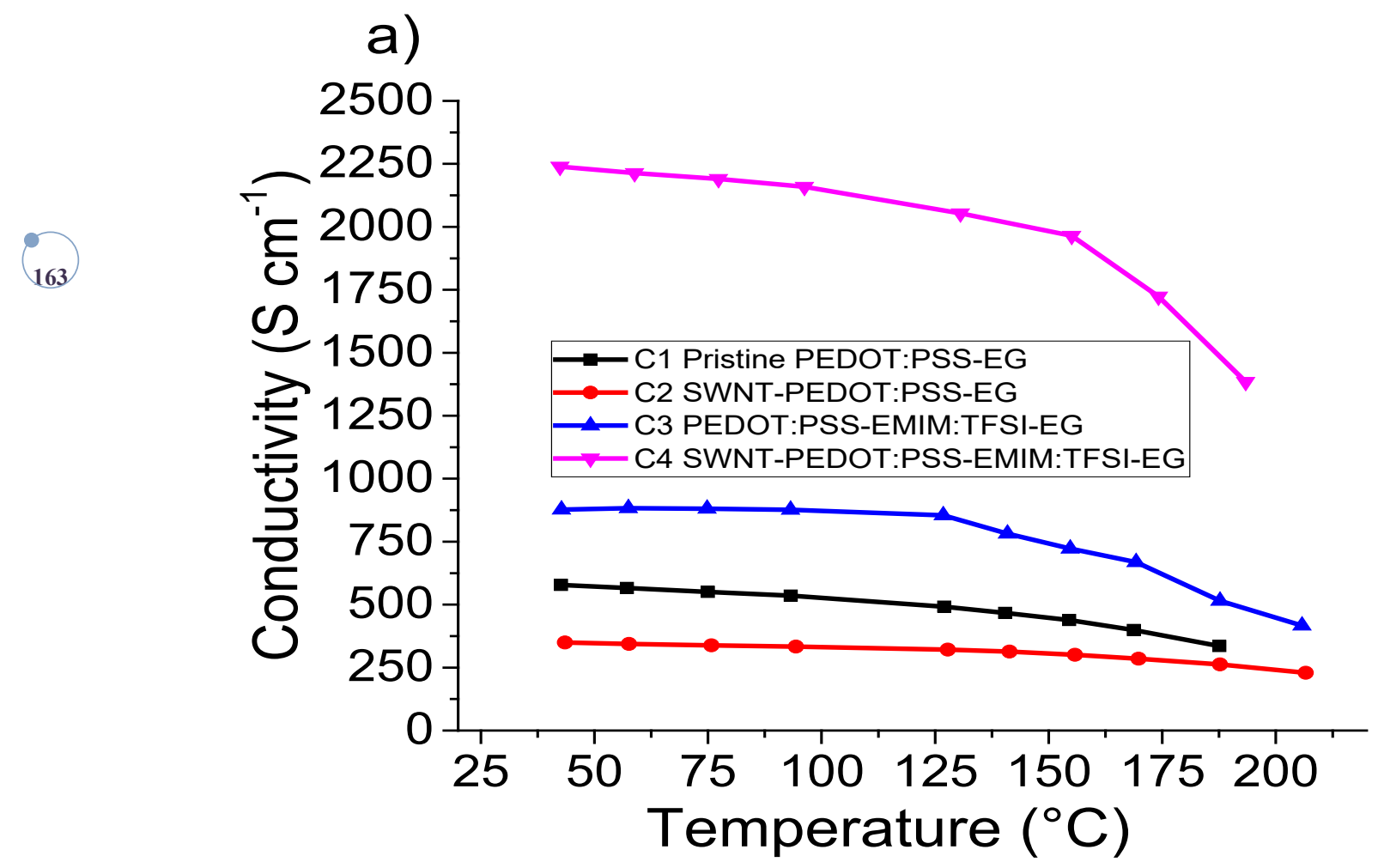

b)

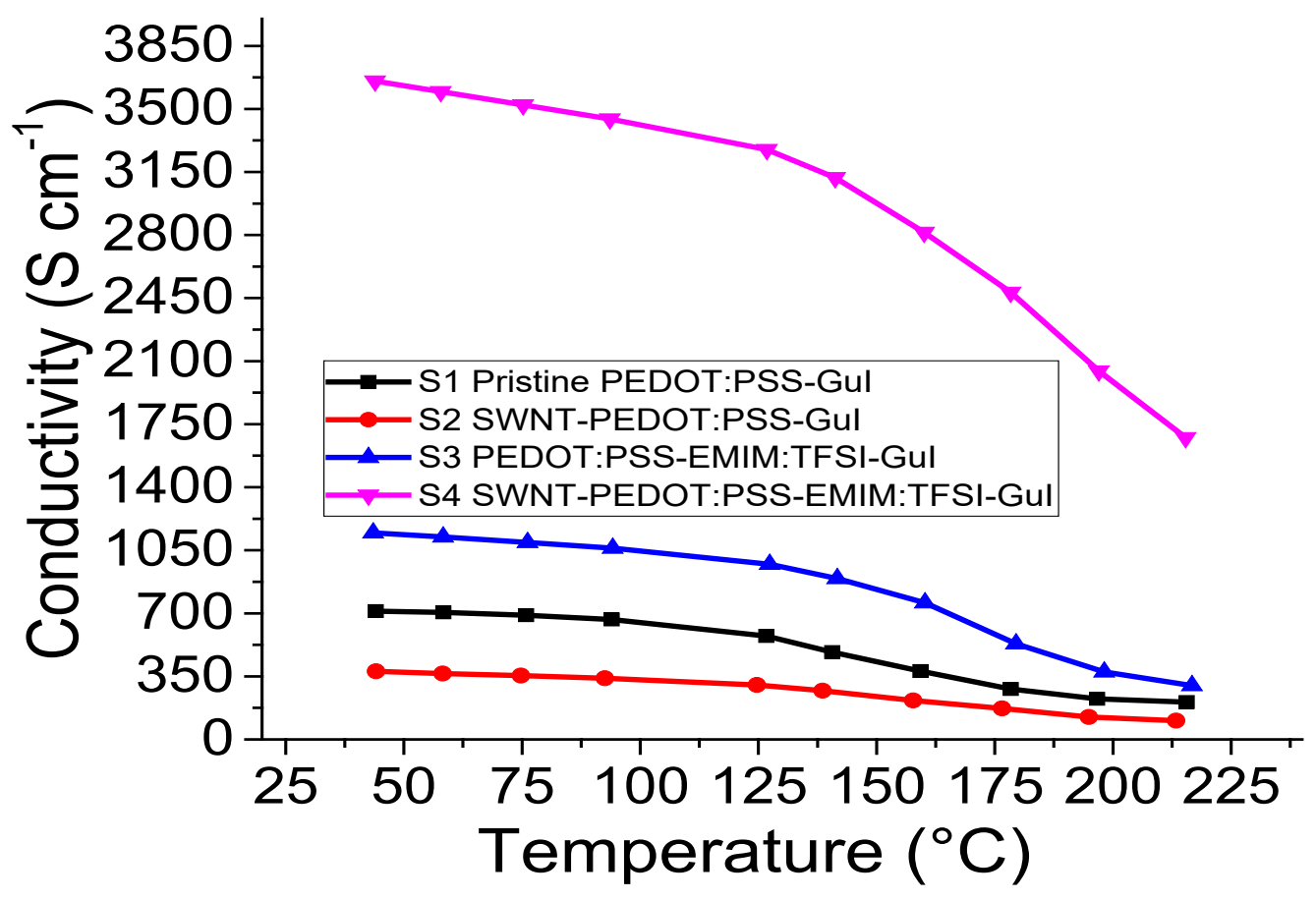

Figure 7.5.2.1 showing the electrical conductivity measured against the temperature profile $\left(40{ }^{\circ} \mathrm{C}\right.$ - $\left.240{ }^{\circ} \mathrm{C}\right)$ for $(\mathrm{a})$, the ethylene glycol controls $(\mathrm{CX})$, and $(b)$. the 
guanidinium iodide treated films whereby guanidinium iodide is dissolved in ethylene $\operatorname{glycol}(S X)$.

The Seebeck coefficient for the FC2 film $\left(20 \mu \mathrm{V} \mathrm{K}^{-1}\right)$ decreased upon ethylene glycol treatment to $13 \mu \mathrm{V} \mathrm{K}^{-1}$ as shown fah or the $\mathrm{C} 2 \mathrm{film}$. This is not unexpected as literature has shown slight decrease in Seebeck coefficient from $37 \mu \mathrm{V} \mathrm{K}^{-1}$ to $<34 \mu \mathrm{V}$ $\mathrm{K}^{-1}$ with ethylene glycol treatment on PEDOT/CNT films. ${ }^{145}$ Still, for the remainder of the films there has been little change to the size of the Seebeck coefficient induced by the ethylene glycol treatment. The only notable difference between the $\mathrm{CX}$ and FCX films is that there is a stronger temperature dependence on the Seebeck coefficient of the CX films. This does infer that the ethylene glycol does change the carrier transport mechanism however it's difficult to make meaningful interpretations without correlating charge carrier mobility and Fermi edge of the composites (beyond scope of this study). ${ }^{58,111,451}$

When analysing Figure 7.5.2.2 (a), the Seebeck coefficient for all CX films is within $13 \mu \mathrm{V} \mathrm{K}^{-1}+/-1.5$ at $40{ }^{\circ} \mathrm{C}$. As shown in Figure 7.5.2.2 (b) the SX films all have a higher Seebeck coefficient than CX films. The lowest Seebeck coefficient for the SX films is the S3 SWNT-PEDOT:PSS-EMIM:TFSI-GuI film in which at $40{ }^{\circ} \mathrm{C}$ is 18.9 $\mu \mathrm{V} \mathrm{K}^{-1}$. The S2 SWNT-PEDOT:PSS-GuI and S1 pristine PEDOT:PSS-GuI films both exhibit a Seebeck coefficient of around $\left(19.8 \mu \mathrm{V} \mathrm{K}^{-1}\right)$.

The highest Seebeck coefficient was attributed to the S4 SWNT-PEDOT:PSSEMIM:TFSI-GuI film which at $40{ }^{\circ} \mathrm{C}$ was recorded as $22 \mu \mathrm{V} \mathrm{K}^{-1}$. Compared to literature the Seebeck coefficient is not high however the improvement is substantial since it has been doubled relative to the FC1 pristine PEDOT:PSS film. In a recent study by Baran et al ${ }^{359}$ the ionic liquid (1- ethyl-3-methylimidazolium-dicyanamide) EMIM:DCI was utilized to as a treatment on PEDOT:PSS. Due to a Seebeck coefficient of $35 \mu \mathrm{V} \mathrm{K}^{-1}$ and a corresponding electrical conductivity of $538 \mathrm{~S} \mathrm{~cm}^{-1}$ a PF of around of $33 \mu \mathrm{W} \mathrm{m}{ }^{-1} \mathrm{~K}^{-2}$ at $123{ }^{\circ} \mathrm{C}$ was obtained. When analysing Figure 7.5.1 (c), and 7.5.2.3 (b) it is clear, with the exception of the FC4 SWNT-PEDOT:PSSEMIM:TFSI film with PF of $50 \mu \mathrm{W} \mathrm{m}{ }^{-1} \mathrm{~K}^{-2}$ at $130{ }^{\circ} \mathrm{C}$; all $\mathrm{CX}$ and $\mathrm{SX}$ films show higher power factor than the remaining FCX films across the temperature range. The reason for the FC4 film's high-power factor is due to its electrical conductivity being the highest regarding the FCX films as well as some CX and SX films. The C4 SWNT- 
PEDOT:PSS-EMIM:TFSI-EG film in turn has the highest power factor for the CX films, as shown with an optimised power factor of $70 \mu \mathrm{W} \mathrm{m}{ }^{-1} \mathrm{~K}^{-2}$ at $130{ }^{\circ} \mathrm{C}$. The film from all the materials with the highest power factor is the S4 SWNT-PEDOT:PSSEMIM:TFSI-GuI in which at the same temperature it is $236 \mu \mathrm{W} \mathrm{m} \mathrm{m}^{-1} \mathrm{~K}^{-2}$. In comparison to films in literature it is a high performing organic thermoelectric. ${ }^{22,232,337}$ In the study whereby ethylene glycol was post treated on a PEDOT:PSS/CNT composites a PF of $151 \mu \mathrm{W} \mathrm{m}^{-1} \mathrm{~K}^{-2}$ was achieved. ${ }^{452}$ 
a)

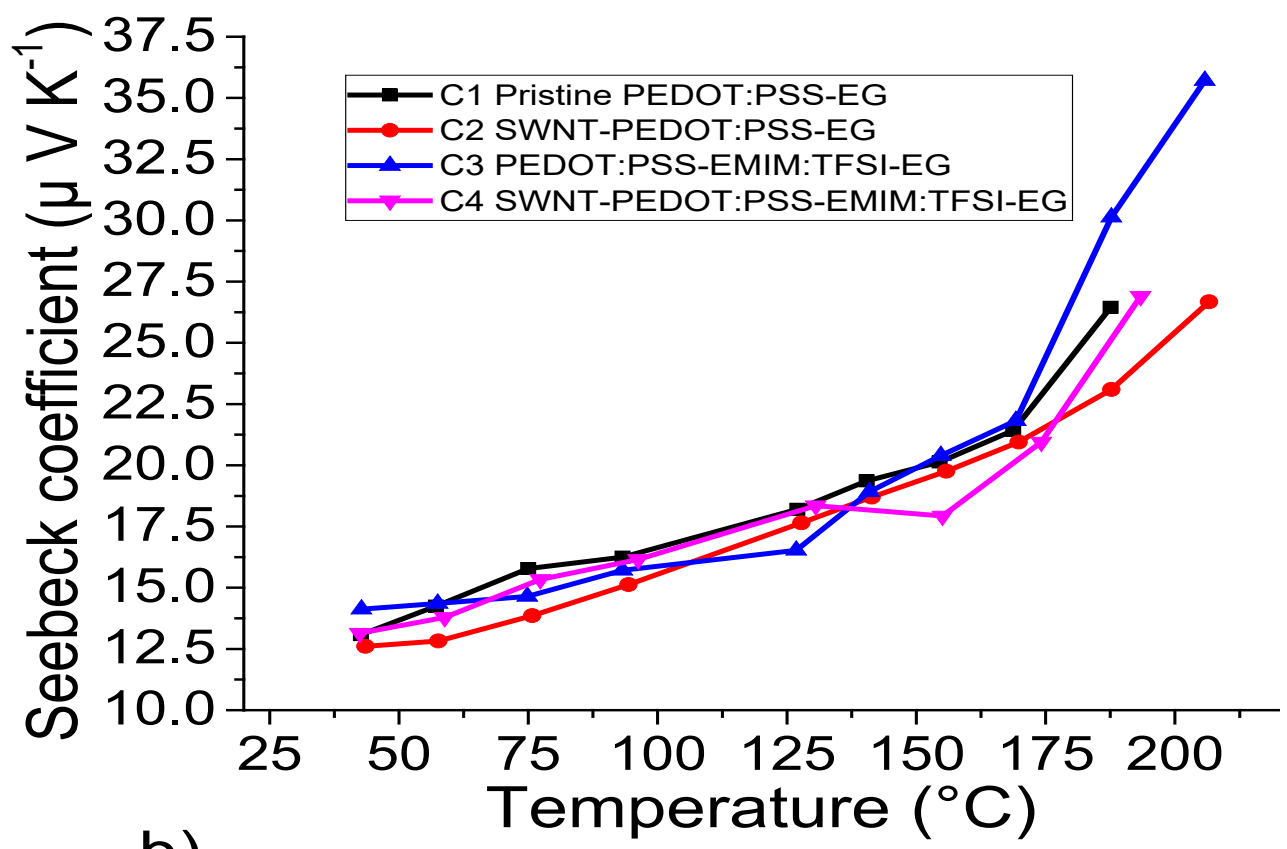

b)

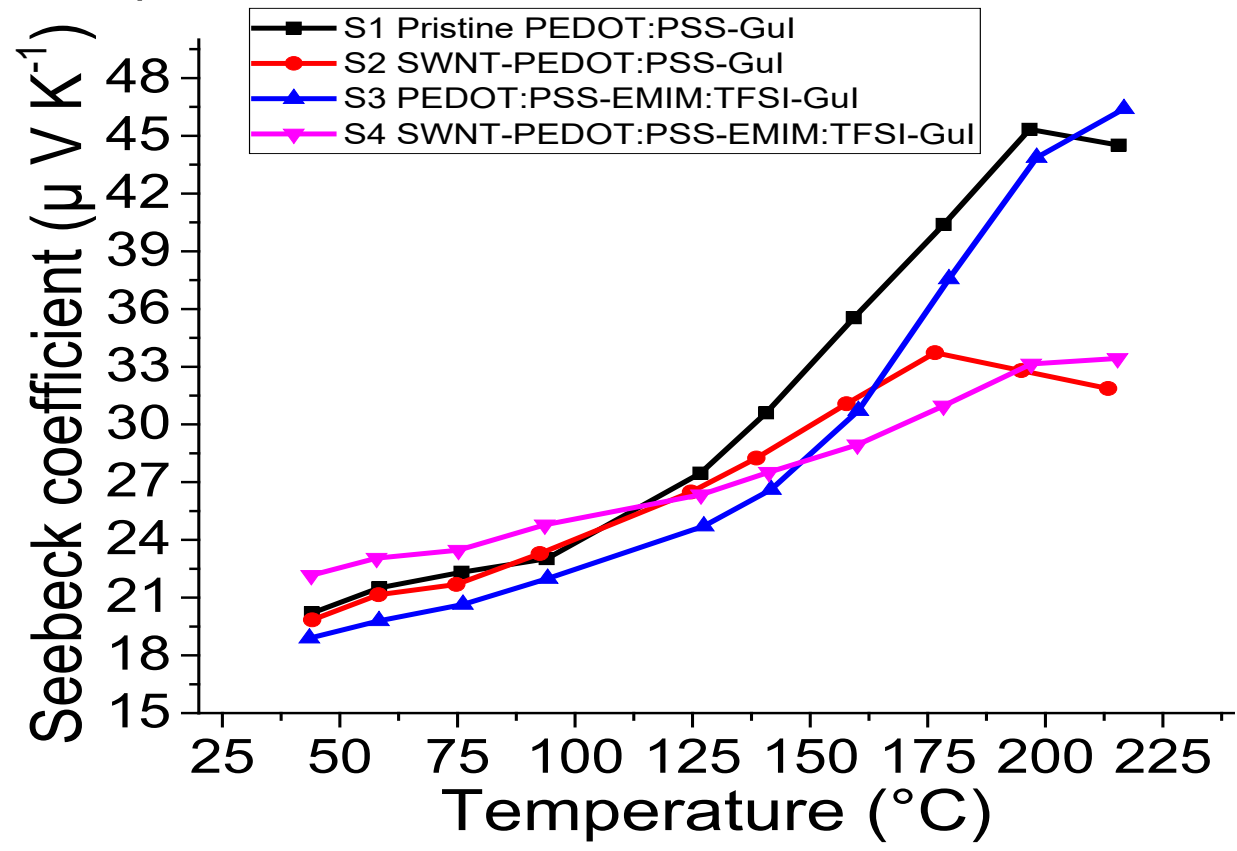

Figure 7.5.2.2 expressing the Seebeck coefficient against a temperature profile (40 ${ }^{\circ} \mathrm{C}-240^{\circ} \mathrm{C}$ ) for (a), the ethylene glycol controls (no guanidinium iodide), and (b). the guanidinium iodide treated films whereby guanidinium iodide is dissolved in ethylene glycol.). 
In a more recent study by $X u$ et al, the ionic liquid 1-butyl-3-methylimidazolium bis (trifluoromethanesulfonyl) imide, (BMIM:TFSI) was used to treat PEDOT:PSS in which the electrical conductivity reached $2783 \mathrm{~S} \mathrm{~cm}^{-1}$ and Seebeck coefficient $14 \mu \mathrm{V}$ $\mathrm{K}^{-1}$ (unaffected) to give a PF of $64 \mu \mathrm{W} \mathrm{m}{ }^{-1} \mathrm{~K}^{-2} \cdot{ }^{367}$ Through base (sodium formaldehyde sulfoxylate) post treatment the PF was optimised to $239 \mu \mathrm{W} \mathrm{m}^{-1} \mathrm{~K}^{-2}$ due to improved $\mathrm{S}\left(61 \mu \mathrm{V} \mathrm{K}^{-1}\right)$ and reduced conductivity $\left(641 \mathrm{~S} \mathrm{~cm}^{-1-}\right)$. Further studies looking into how the concentration of the SWNT, and or concentration of the GuI solution it may be possible to optimise the PF of these novel OHTEG composites developed in this study.

a)

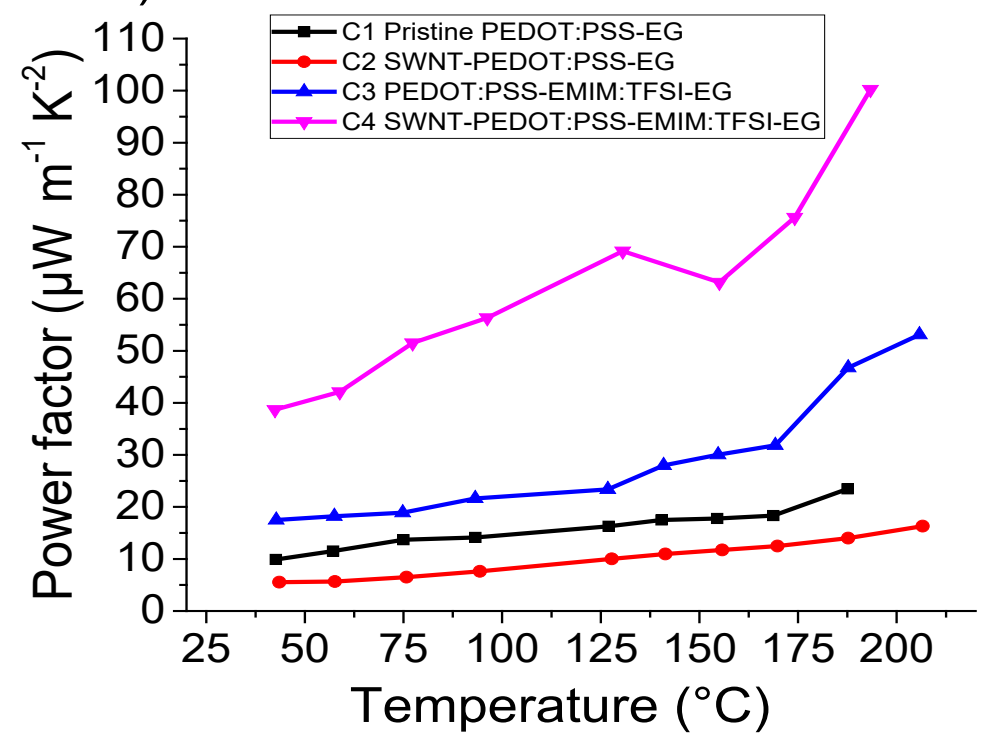

b)

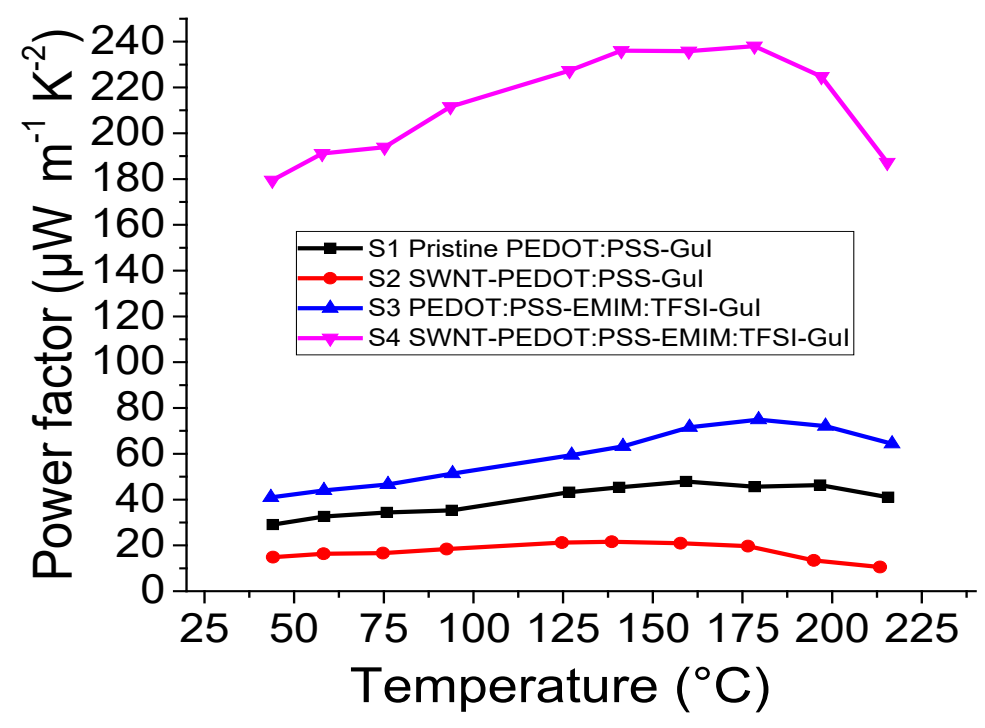


Figure 7.5.2.3 expressing the Power factor against a temperature range $\left(40{ }^{\circ} \mathrm{C}\right.$ - 240 $\left.{ }^{\circ} \mathrm{C}\right)$ for (a), the ethylene glycol controls $(\mathrm{CX})$ and, (b) the guanidinium iodide treated films whereby guanidinium iodide is dissolved in ethylene glycol (SX).

\subsubsection{Raman spectroscopy of the FC1, CX and SX films}

Figure 7.5.3.1 depicts the Raman spectra for the FC1, CX and SX films. As discussed in previous experimental chapters, Raman spectroscopy can be used to study the conformation (benzoid or quinoid) of majority of the polythiophenes in PEDOT:PSS via the position of symmetrical stretching of the $\mathrm{C}_{\alpha}=\mathrm{C}_{\beta}$ typically found around $1440 \mathrm{~cm}^{-1}$ for pristine PEDOT:PSS. ${ }^{298,300,361,372}$ A red shift indicates a conformational change from benzoid to quinoid in which the thiophene molecules adapt a more planer structure due to more restrictive movement along the double bond backbone, meanwhile in the benzoid structure many thiophene molecules adopt a helical structure. ${ }^{299,338,453}$ Figure 7.5.3.2 shows the different conformation of polythiophenes whereby (a) denotes the quinoid and (b) denotes the benzoid structure. ${ }^{453}$ As indicated by the position of the double bond it's understandable that the quinoid structure will have more restrictive rotation thereby leading to more structured and ordered planner structure where the thiophenes will have stronger $\pi-$ $\pi$ interactions and shorter stacking distance thereby leading to improved carrier mobility due to more linear chains. 227,246,357,361

The FC1 film represents the pristine PEDOT:PSS film used as an untreated reference spectra to compare other treated films to. The corresponding Raman shift is seen at $1440 \mathrm{~cm}^{-1}$ as shown in Figure 7.5.3.1 and Table 7.5.3.1. As seen in the Figure and Table, all films exhibit a red shift albeit by different amounts. This indicates that some polythiophenes have re arranged their conformation towards the quinoid structure as depicted in Figure 7.5.3.2 (a). As was discussed in chapter 7.5.2 and specifically from the data exhibited in Figure 7.5.2.1 all films treated with ethylene glycol (CX), as well as all films treated with the GuI solution (SX) showed substantial increase in the electrical conductivity.

In general, it appears that the SX films except for the S1 film have a more significant red shift than CX films. This does indicate that there is a potential that the SX films have more thiophenes molecules in the quinoid conformation however, the red shift 
has also been mapped to include an inference of reduction (dedoping) which is usually coupled with more intense narrow peaks at the symmetrical stretching of the $\mathrm{C}_{\alpha}=\mathrm{C}_{\beta}$ region. In this case it is difficult to make a conclusion that the peak for the SX films have narrowed more than for the CX films unlike was seen for the films treated with $\mathrm{NaBH}_{4}$ in chapter 5.3.4 Figure 5.3.4.2. ${ }^{2}$ Therefore the extent of the Raman shift for the SX films indicates only slight reduction.

As shown in Figure 7.5.3.1 (a) at $1591 \mathrm{~cm}^{-1}$ is a peak labelled CNT G band. In graphitic structures such as SWNT the G band and a corresponding D band is usually found between (1580-1600 $\left.\mathrm{cm}^{-1}\right)$ and (1340-1360 $\left.\mathrm{cm}^{-1}\right)$ respectively. ${ }^{297,454,455}$ The D band is characterized as the disorder band and the higher the ratio between the $\mathrm{G}$ and D the more ordered (less structural defects) the graphitic structures exhibits. ${ }^{456}$ It's also possible to infer if $\mathrm{p}$ or n-type doping has occurred in the SWNT by the shift at the G band whereby a red shift can be linked to an $\mathrm{n}$ type doping (electron injection) and blue shift can represent $\mathrm{p}$ type doping. ${ }^{412,457,458}$ Figure 7.5.3.3 depicts a SWNTPEDOT:PSS film treated with $60 \%$ SWNT instead of the $40 \%$ SWNT concentration used for all other films in this chapter. This is because as shown in Figure 7.5.3.1 at the region labelled CNT G band, the signal is convoluted with the peak region for PSS. 
a) Red shift indicating bezoid to quinoid conformational shift

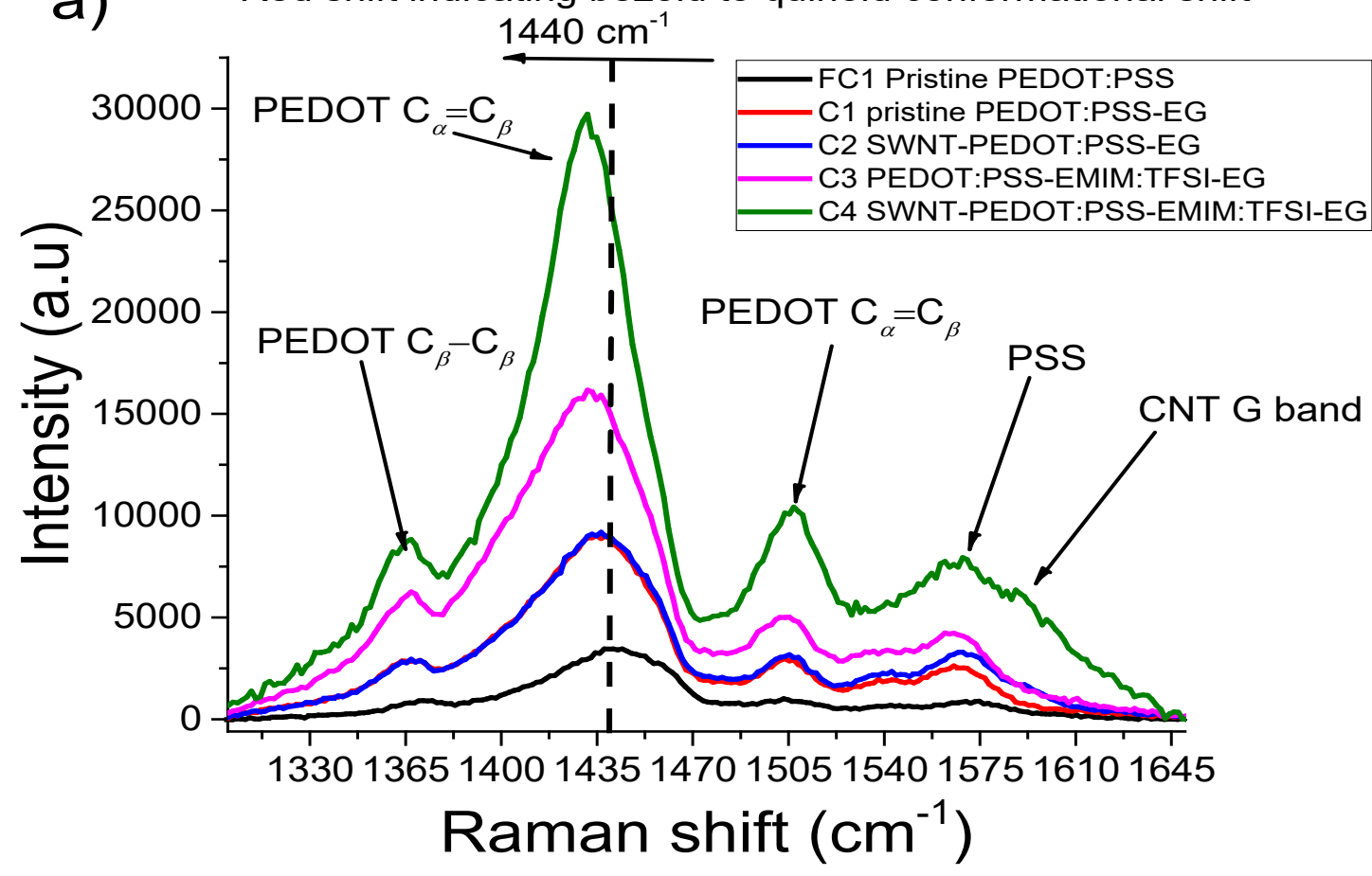

b) Red shift indicating bezoid to quinoid conformational shift

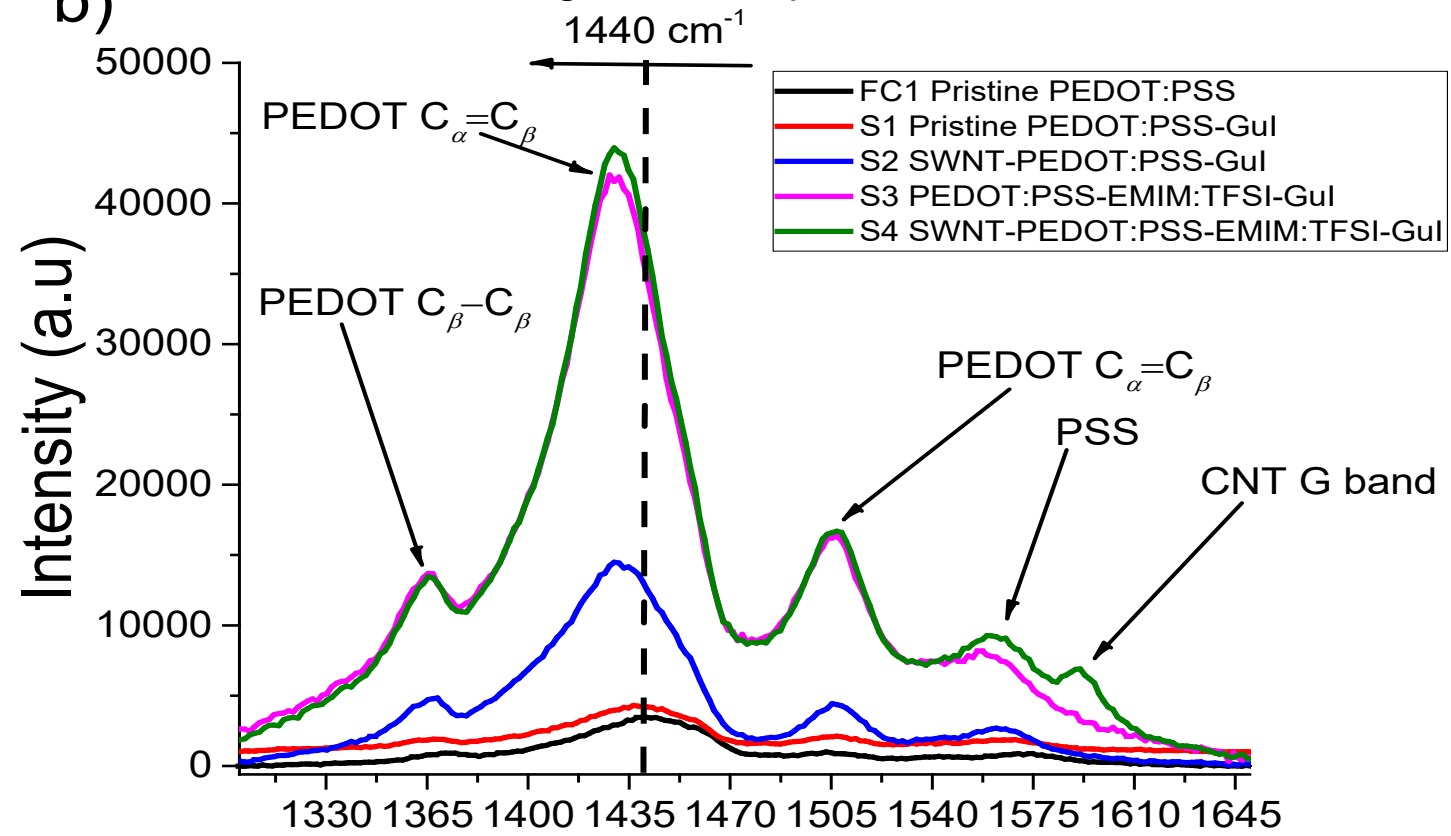

Raman shift $\left(\mathrm{cm}^{-1}\right)$

Figure 7.5.3.1 depicting the benzoid to quinoid shift at $1440 \mathrm{~cm}^{-1}$ for (a) the CX films and (b) the SX films. (FC1 pristine PEDOT:PSS in in both spectra as a baseline for the shift). 
a) neutral quinoid

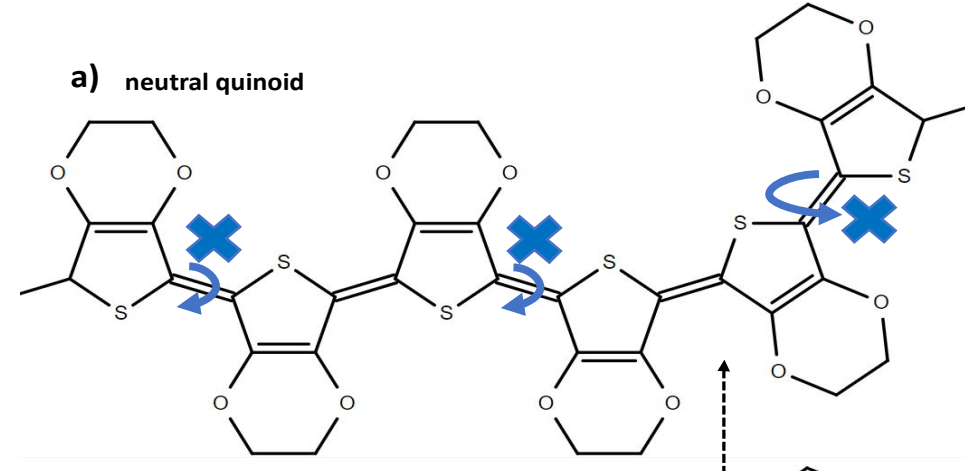

b) neutral benzoid<smiles>Cc1sc(-c2scc3c2OCCO3)c2c1OCCO2</smiles>

c) positive polaron

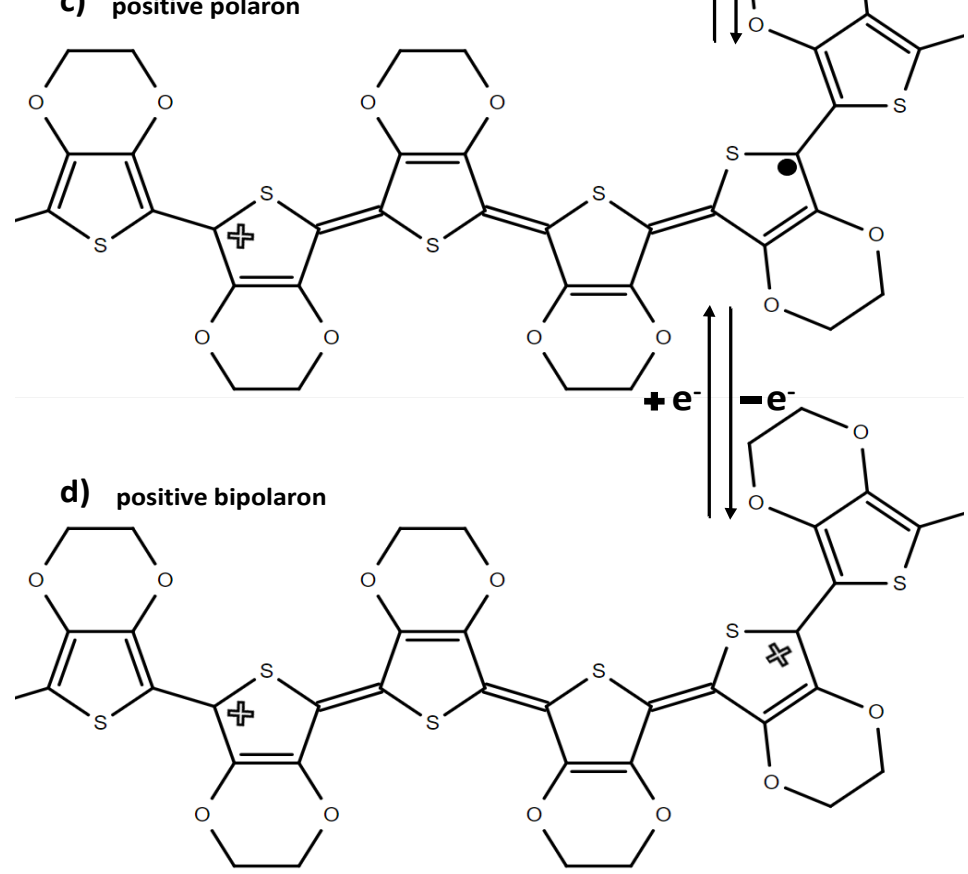

Figure 7.5.3.2 showing different resonance and oxidation state of PEDOT backbone. (a) is the neutral quinoid structure in which rotation of carbon is more restricted meanwhile (b) is the benzoid structure where rotation is allowed. (c) Corresponds to 
the singly positively charged form of PEDOT and (d) the doubly positively charged form.

Table 7.5.3.1 depicting the benzoid to quinoid shift for the FC1, CX and SX films regarding the $C_{\alpha}=C \beta$ symmetric stretching found at $1440 \mathrm{~cm}^{-1}$.

\begin{tabular}{|l|c|}
\hline Film & $\begin{array}{l}\text { Raman shift }\left(\mathrm{cm}^{-1}\right) \text { for the symmetric } \mathrm{C}_{\alpha} \\
=\mathrm{C}_{\beta} \text { stretching }\end{array}$ \\
\hline FC1 pristine PEDOT:PSS & 1440.0 \\
\hline C1 pristine PEDOT:PSS-EG & 1434.8 \\
\hline C2 SWNT-PEDOT:PSS-EG & 1436.4 \\
\hline C3 PEDOT:PSS-EMIM:TFSI-EG & 1431.5 \\
\hline C4 SWNT-PEDOT:PSS-EMIM:TFSI- & 1431.5 \\
EG & 1436.4 \\
\hline S1 pristine PEDOT:PSS-GuI & 1429.8 \\
\hline S2 SWNT-PEDOT:PSS-GuI & 1428.2 \\
\hline S3 PEDOT:PSS-EMIM:TFSI-GuI & 1429.8 \\
\hline S4 SWNT-PEDOT:PSS-EMIM:TFSI- & \\
GuI & \\
\hline
\end{tabular}




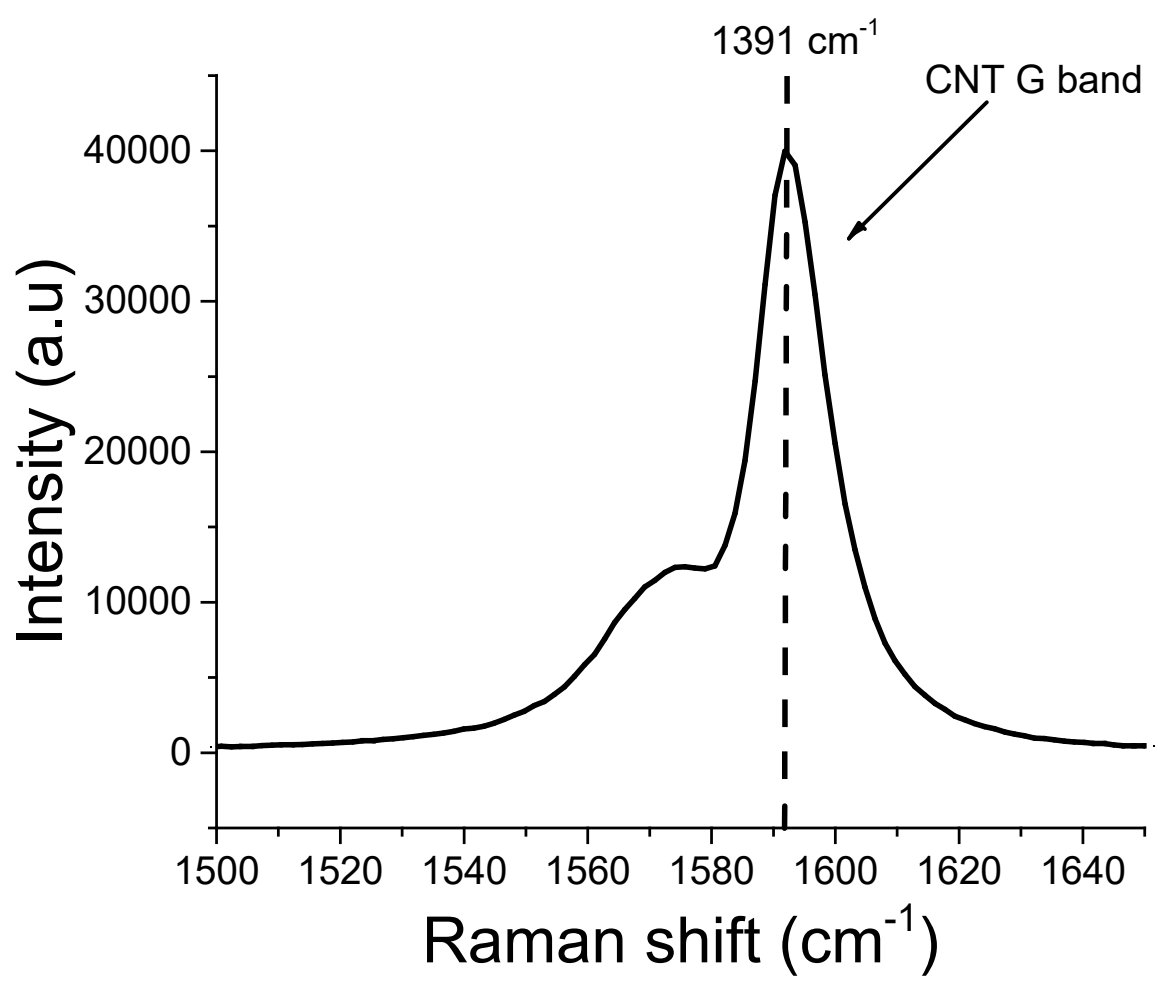

Figure 7.5.3.3 depicting the G band for the SWNT-PEDOT:PSS film with 60\% SWNT content instead of $40 \%$ which is present for the films studied in this section (chapter7.5.3). This is a control to elucidate is $p$ or $n$ doping has occurred.

When analysing Figure 7.5.3.1 (a) and (b), at $1591 \mathrm{~cm}^{-1}$ there is a distinct peak for the S4 films meanwhile the $\mathrm{C} 4$ film has a peak that is less distinct. The presence of the peak indicates the presence of SWNT in the film. ${ }^{412,459,460}$ It is however not possible to tell if the S2 and C2 films had a peak at the area because the signal from the SWNT in those films is small as well as stretching vibrations in the same region as PSS. None of the films without known SWNT content had a distinct or otherwise identifiable peak at around $1591 \mathrm{~cm}^{-1}$. The $\mathrm{D}$ band is typically found at $1340 \mathrm{~cm}^{-1}$ therefore even for the S4 film it is not possible to identify the peak due to it being in the same region as PEDOT'S $\mathrm{C}_{\beta}-\mathrm{C}_{\beta}$ vibration therefore it's not possible to understand the extent of SWNT disorder. ${ }^{456,461,462}$ When comparing the peak position for the S4 film at 1591 $\mathrm{cm}^{-1}$, it is in the same position as the control films peak $\left(1591 \mathrm{~cm}^{-1}\right)$ as shown in Figure 
7.5.3.3. This suggests the guanidinium iodide has not cased significant $\mathrm{p}$ or $\mathrm{n}$ doping that can be detected by a Raman shift at the G band.

\subsubsection{Proposed mechanisms for increased Seebeck coefficient}

To determine some origin behind the improved Seebeck coefficient, UV-Vis-NIR absorption spectra were obtained for the CX and SX films. ${ }^{463-465}$ As shown below in Figure 7.5.4.1 are the UV-Vis-NIR spectra for the CX films depicting the wavelength for thiophene excitation at three distinct regions and an extra region labelled $\mathrm{I}_{3}-{ }^{-466,467}$ Figure 7.5.4.2 depicts the UV-Vis-NIR spectra for the SX films.

In literature a reduction of charge carriers as indicated by an emergence or increased quantity of polarons or neutral thiophene absorption intensity is usually accompanied by improved Seebeck coefficient in PEDOT:PSS based films. ${ }^{128,468-471}$ As shown in Figure 7.5.4.1 The C3 PEDOT:PSS-EMIM:TFSI-EG and C4 SWNT-PEDOT:PSSEMIM:TFSI-EG film exhibited the highest maximum absorbance above $1800 \mathrm{~nm}$. This is most probably due to the films being thicker than the $\mathrm{C} 1$ and $\mathrm{C} 2$. The difference being due to both $\mathrm{C} 3$ and $\mathrm{C} 4$ films having had the ionic liquid treatment which subsequently increased viscosity of solution thereby leading to slightly thicker films when spun. The strong absorption above $1800 \mathrm{~nm}$ and the lack of a distinct absorption at $900 \mathrm{~nm}$ or $600 \mathrm{~nm}$ for all CX films indicates majority of thiophenes have bipolarons as charge carriers. ${ }^{194,308}$ (Please see Figure 7.5.3.2 (d). This suggests that the CX films did not undergo substantial thiophene reduction via ethylene glycol treatment. This is corroborated by the lack of improved Seebeck coefficient for the CX films relative to the FC1 pristine PEDOT:PSS as shown when comparing Figure 7.5.1 (b) and 7.5.2.2 (a). 


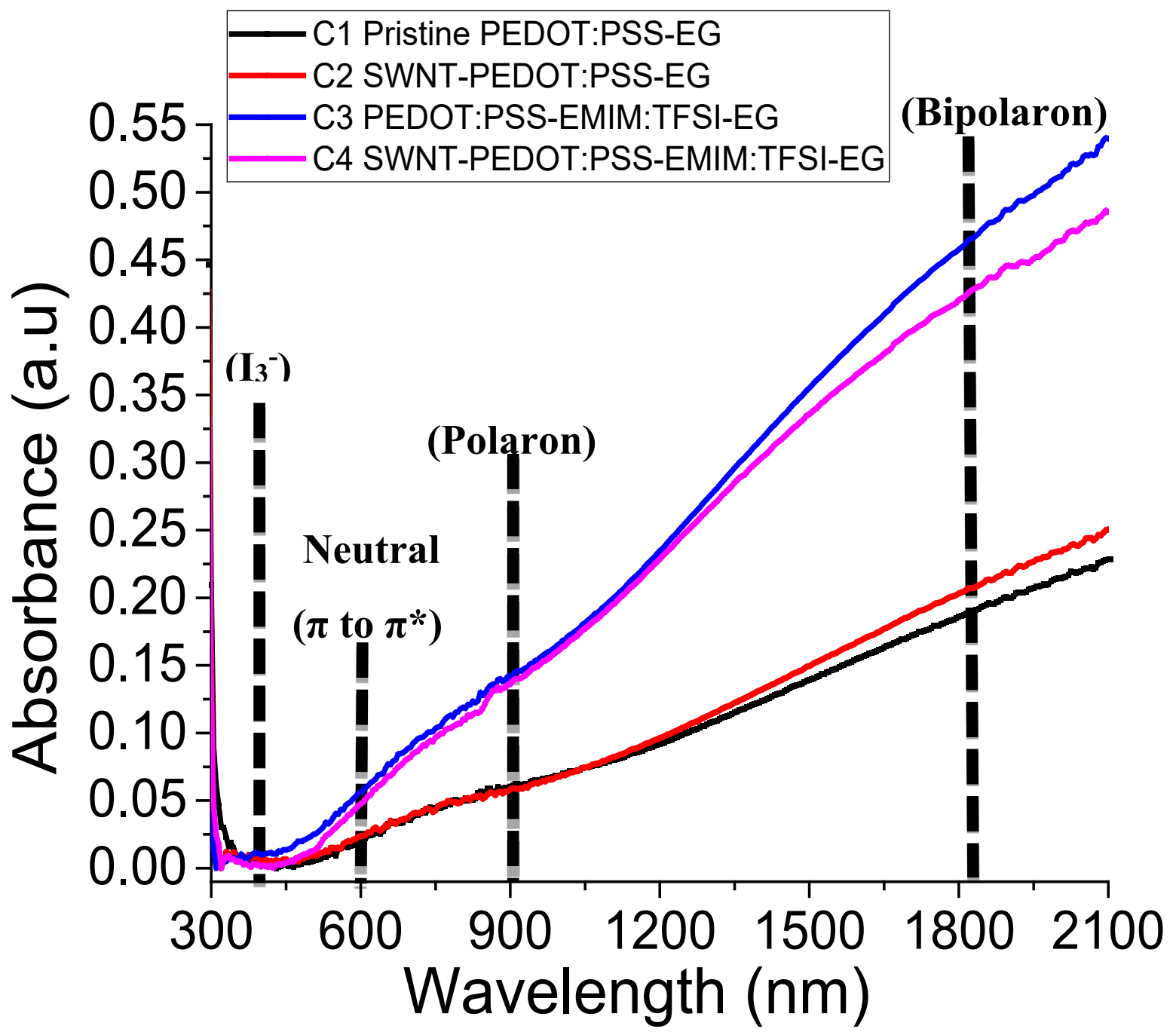

Figure 7.5.4.1 depicting the UV-Vis-NIR spectra for the CX films across $300 \mathrm{~nm}$ to $2100 \mathrm{~nm}$. At around $385 \mathrm{~nm}$ is the suspected absorbance of residual $I_{3}{ }^{-}$after Iodide undergoes a redox reaction with some thiophene molecules. At around $600 \mathrm{~nm}$ is the $\pi$ to $\pi^{*}$ transition (neutral) meanwhile around $900 \mathrm{~nm}$ depicts the singly charged polaron, and above $1800 \mathrm{~nm}$ characterises the absorption for bipolarons in thiophenes backbone. ${ }^{468}$ 


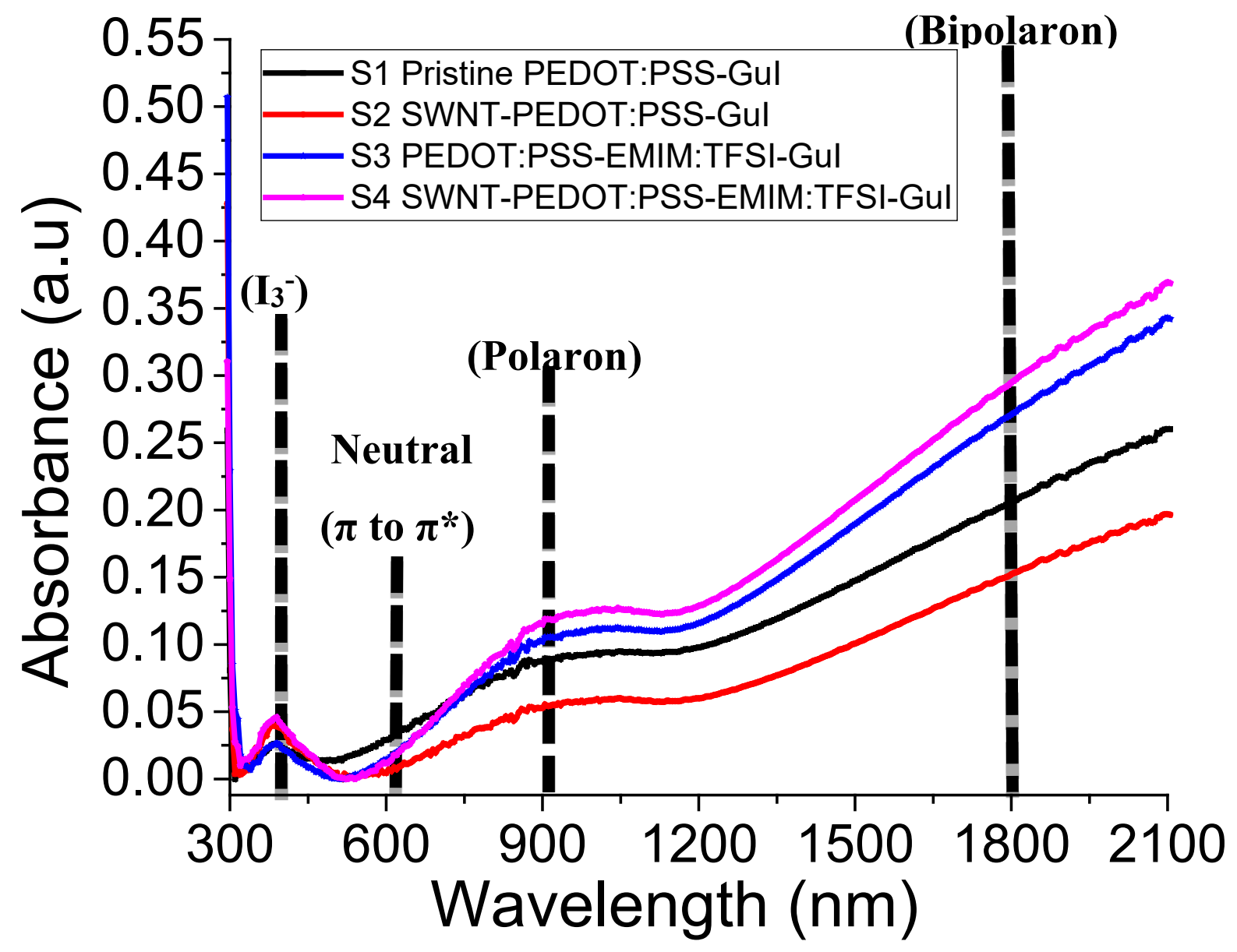

Figure 7.5.4.2 showing the UV-Vis-NIR spectra for the SX films across $300 \mathrm{~nm}$ to 2100 $\mathrm{nm}$. At around $385 \mathrm{~nm}$ is the suspected $\mathrm{I}_{3}^{-}$absorption after Iodide undergoes redox reaction with some thiophene molecules. At around $600 \mathrm{~nm}$ is the $\pi$ to $\pi^{*}$ transition (neutral) meanwhile around $900 \mathrm{~nm}$ depicts the singly charged polaron absorption and above $1800 \mathrm{~nm}$ is the bipolaron absorption on the thiophene backbone. ${ }^{463,472}$

In comparison to the $\mathrm{CX}$ films the, $\mathrm{SX}$ films as depicted in Figure 7.5.4.2 appear to have undergone some thiophene reduction. The S3 and S4 films have the highest absorbance across the measured range and similarly to the $\mathrm{C} 3$ and $\mathrm{C} 4 \mathrm{films}$, both films were slightly thicker than the S1 and S2 films with lower maximum absorbance. ${ }^{473}$ What is notable and different compared to the CX films is the emergence of a distinct peak at $900 \mathrm{~nm}$ for all SX films. ${ }^{128}$ (Please refer to Figure 7.5.3.2 (c) for representation of polaron charge carrier in the thiophene backbone. ${ }^{98,212}$ The emergence of the polaron peak intensity as well as the improved Seebeck coefficient as shown for all SX films (Figure 7.5.2.2 (b)) with the treatment of GuI 
solution indicates that the GuI solution is responsible. It is of note that there is no distinct peak arising for any of the SX films at $600 \mathrm{~nm}$. This means that the films are not reduced to their neutral state thus indicating under the GuI treatment conditions (Please refer to chapter 7.4.6) reduction is stopped at polaron formation.

Consequently, this suggests that the SX films' primary charger carriers are bipolarons (largest relative absorption area in each film) with some thiophene molecules harbouring polaron charge carriers. ${ }^{474,475}$ In reference to chapter 7.2 Equation 2; since each SX film had higher electrical conductivity than the CX equivalent despite the observed reduced charge carriers in the SX films (Figure 7.5.2.2 (b) one of two or an interplay between both possibilities are at hand. The first is that there is significantly improved carrier mobility in the SX films (more than in CX films) thus explaining the improved electrical conductivity despite the loss of charge carriers. This, however, is difficult to confirm due to not having taken Hall effect measurement for bulk carrier mobility of each film. The Raman studies in chapter 7.5.3 Figure 7.5.3.1 indicated improved polymer orientation for better carrier transport for all CX and SX films via observed red shift at the $\mathrm{C}_{\alpha}=\mathrm{C}_{\beta}$ symmetrical stretching vibration. ${ }^{476}$ The second possibility is that the UV-Vis-NIR spectra only indicates reduction of charge carriers along the thiophene backbones and that possibly other charge carriers may be adsorbed on the surface such as residual guanidinium ions. In literature it is shown, surface ion accumulation can contribute in improving Seebeck coefficient and electrical conductivity of PEDOT and other organic films. ${ }^{47-482}$

To illustrate the quantitative change in absorbance based on reduction of thiophene molecules, ${ }^{468}$ Figure 7.5.4.3 (a) represents the UV-Vis-NIR spectra for the C1 pristine PEDOT:PSS-EG and S1 pristine PEDOT:PSS-GuI film. Because both films had similar thicknesses $55 \mathrm{~nm}(\mathrm{~S} 1)$ and $58 \mathrm{~nm}(\mathrm{C} 1)$, it is possible to compare absorbance. As shown the bipolaron region in the $\mathrm{C} 1$ film is the highest region with no distinct intensity at the polaron or neutral region. When the film is treated with GuI however at $900 \mathrm{~nm}$ an appearance of polaron peak intensity emerges with a corresponding loss of intensity at the bipolaron region. ${ }^{315,337,356,483}$. Interestingly at $385 \mathrm{~nm}$ a distinct peak is observed and present for all the SX films (Figure 7.5.4.2 and Figure 7.5.4.3 (b) meanwhile not present in any of the CX films (Figure 7.5.4.1). The $177 \mid \mathrm{P}$ a g e 
peak cannot represent any excited SWNT, or EMIM:TFSI because it is present even for the S1 pristine PEDOT:PSS film. It is also very unlikely for the peak to represent thiophene excitation for a negatively charged species on the thiophene backbone. This is because as concluded from analysis of Figure 7.5.4.2 none of the SX films showed an emergence of a neutral peak intensity at $600 \mathrm{~nm}$. For a film to harbour significant amount of negatively charged carriers it would first require a significant amount of thiophenes to be in the neutral phase. 

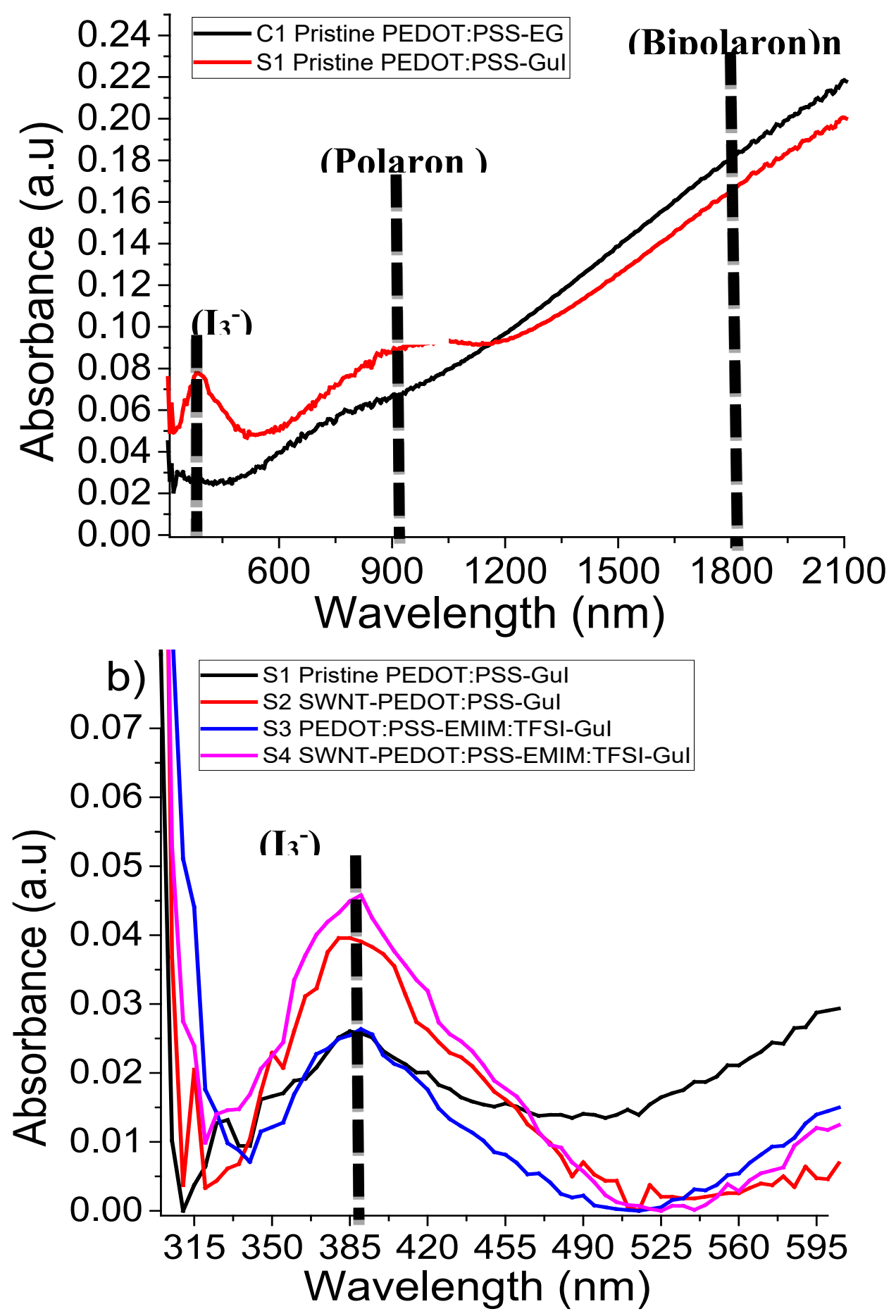

Figure 7.5.4.3 (a) depicting the UV-Vis-NIR spectra for the C1 pristine PEDOT:PSSEG, and S1 pristine PEDOT:PSS-GuI from 300-2100 nm. (b) Depicts the UV-Vis 
spectra for suspected residual $I_{3}^{-}$anions on the film after $I^{-}$anions have undergone a redox reaction with some thiophenes between $(310-500 \mathrm{~nm})$.

The most convincing argument is that the peak at $385 \mathrm{~nm}$ represents residual iodide species adsorbed on the films' surface. It is argued that based on the confirmed thiophene reduction for SX films (Figure 7.5.4.2) the product of the redox reaction $\left(\mathrm{I}_{3}{ }^{-}\right.$ ) is responsible. Equation 5 below depicts the dissociation of GuI to its conjugate acid and base pair. ${ }^{484}$ The guanidinium ion $\left(\mathrm{Gu}^{+}\right)$can donate a $\mathrm{H}^{+}$in a reaction ${ }^{485,486}$ (discussed later in chapter 7.6), meanwhile the $\mathrm{I}^{-}$contributes an electron to reduce a thiophene molecule as seen for Iodine doping on polyaniline. ${ }^{487-491}$ Equation 6 depicts the proposed equation for the redox reaction. ${ }^{492}$

$3 \mathrm{I}^{-}+2 \mathrm{PEDOT}^{2+} \rightarrow \mathrm{I}_{2}+2 \mathrm{PEDOT}^{+}+\mathrm{I}^{-}$

$\mathrm{I}_{2}+\mathrm{I}^{-} \rightarrow \mathrm{I}^{-}$

$2 \mathrm{PEDOT}^{+}+\mathrm{I}_{3}^{-}$

Various iodine species in different oxidation states have been reported in literature between $180 \mathrm{~nm}$ and $500 \mathrm{~nm} .{ }^{493-495}$ Studies looking into the redox reaction between iodine and iodide species have detected $\mathrm{I}^{-}$at around $293 \mathrm{~nm}$, ( $\mathrm{I}_{2}$ around $290 \mathrm{~nm}$ and $460 \mathrm{~nm}$ ) and $\mathrm{I}_{3}{ }^{-}$at around $350 \mathrm{~nm}$ and $366 \mathrm{~nm}$ (slight difference in absorption maxima indicate difference in chemical environment). ${ }^{466,493,496}$ Therefore the peak at $385 \mathrm{~nm}$ isn't too different from $366 \mathrm{~nm}$ and the difference may be due to possibility of the $\mathrm{I}_{3}{ }^{-}$ being in a different chemical environment/ionic interaction with $\mathrm{PEDOT}^{+}$. (Please note it was not possible to get quality data under $280 \mathrm{~nm}$ in the UV-Vis-NIR instrument used therefore complex comparisons between the redox states of iodide was not conducted). For future work it would be interesting to do a kinetic study and measure UV-Vis-NIR between $150 \mathrm{~nm}$ to $2100 \mathrm{~nm}$ for redox and kinetic studies of Iodide thiophene reactions to establish controlled redox to improve Seebeck coefficient for

$\mathbf{1 8 0} \mid \mathrm{P}$ a g e 
optimised PF. ${ }^{497}$ It is of note that $\mathrm{I}_{2}$ treatment even at thermal conditions $160{ }^{\circ} \mathrm{C}$ on PEDOT:PSS did not improve Seebeck coefficient as was explored in some preliminary experiments.

Bellow in Figure 7.5.4.5 (d) is the proposed mechanism of partial reduction (bipolaron to polaron) of polythiophene by the $\mathrm{I}^{-}$anion (see Equation 5 and 6). ${ }^{492}$
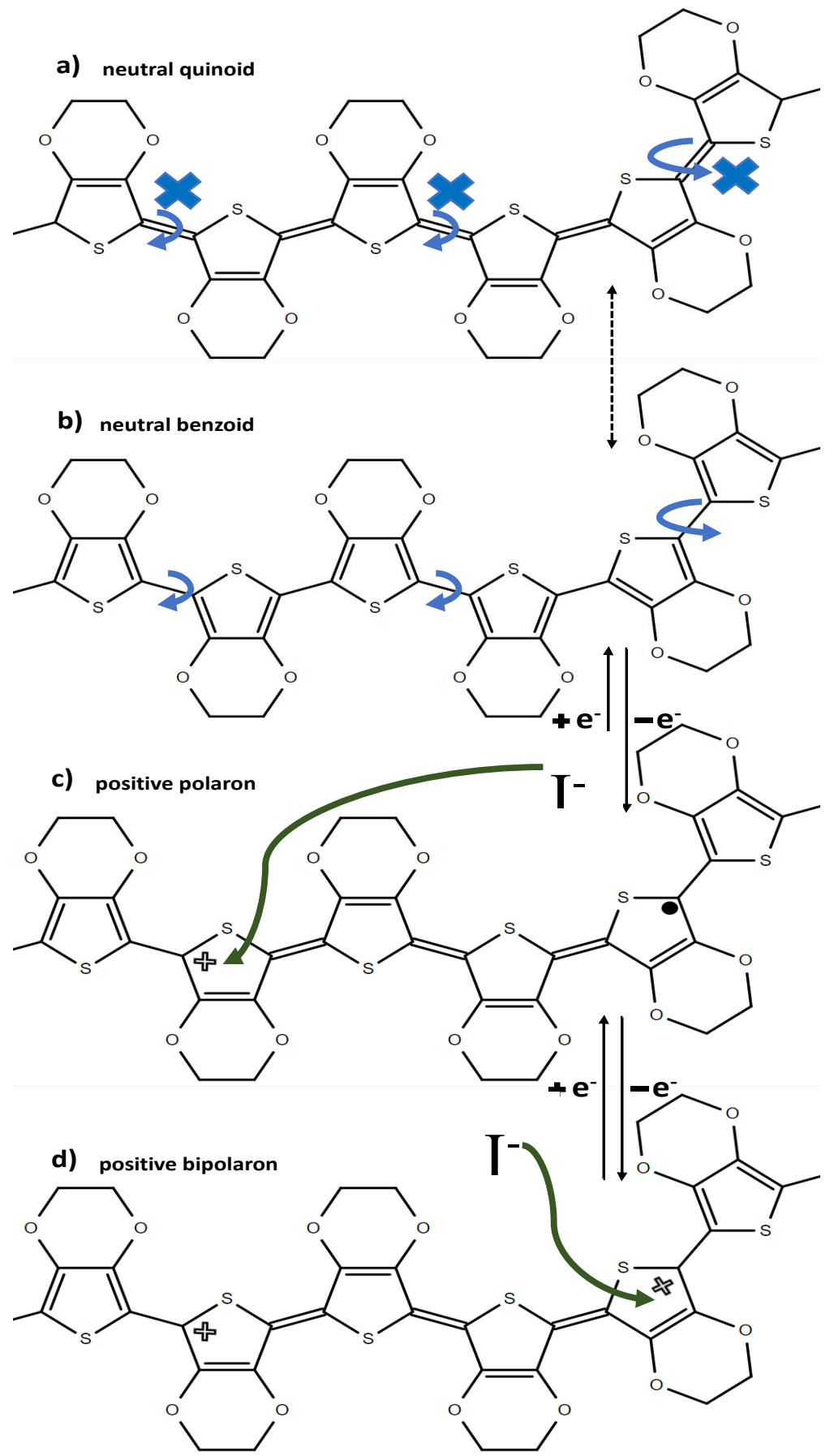
Figure 7.5.4.5 showing different resonance and oxidation state of PEDOT backbone. (a) is the neutral quinoid structure in which rotation of carbon is more restricted meanwhile (b) is the benzoid structure where rotation is allowed. (c) Corresponds to the singly positively charged form of PEDOT and (d) the doubly positively charged form. The I' denotes the Iodide anions in solution and the arrows represent mechanism of reduction on the PEDOT backbone.

The dissociated $\mathrm{I}^{-}$injects an electron in the thiophene backbone to reduce the bipolaron into a polaron (Figure 7.5.4.5 (c). After $2 \mathrm{I}^{-}$species inject $2 \mathrm{e}^{-}$to reduce 2 $\mathrm{PEDOT}^{2+}$ units to $2 \mathrm{PEDOT}^{+}$units, they bond to form $\mathrm{I}_{2}$ whereby the $\mathrm{I}_{2}$ bonds with a non-reacted $\mathrm{I}^{-}$species to form the $\mathrm{I}_{3}{ }^{-}$species observed. ${ }^{492}$ Iodide redox reactions can be seen elsewhere in research. ${ }^{498-502}$ The fact that even at $160{ }^{\circ} \mathrm{C}$ treatment for 20 minutes $\mathrm{I}^{-}$reduction only produced polarons suggests that the limiting factor for further reduction may be concentration. Further studies looking at concentration of GuI solution under same thermal conditions will allow for optimised Seebeck coefficient.

Figure 7.5.4.6 depicts the overlaid XPS spectra for the FC1, CX and SX films. It is extensively seen in literature that a reduction in the polythiophene backbone can be detected via a chemical shift towards lower binding energy (BE) regarding the PEDOT's sulphur 2p Spin-orbital split (S 2p), (typically found around 164 eV). ${ }^{350,365,373,407,503,504}$ As can be seen, there are three distinct regions as labelled. The furthest peak to the left beyond $168 \mathrm{eV}$ corresponds to the PSS's sulfonate and sulfonic acid's S 2 p signal meanwhile the peak around $164 \mathrm{eV}$ corresponds to PEDOT's thiophene S 2p signal. ${ }^{187,196,505}$ Surprisingly, there is also an a peak at $162.5 \mathrm{eV}$ not usually seen in literature entitled $\mathrm{SH}_{2}$ (explored later in chapter 7.6). ${ }^{307,506-510}$ It is evident that the CX and SX films have considerably lower PSS to PEDOT ratio relative to the FC1 pristine PEDOT:PSS film (explored in chapter 7.6).

When analysing Figure 7.5.4.6 and Table 7.5.4.2 it is evident that all SX films exhibit a chemical shift towards a lower BE at the $\mathrm{S} 2 \mathrm{p}$ PEDOT region relative to the control film (FC1). The S2 film has the smallest change in $\mathrm{BE}(\Delta \mathrm{BE})$ of $0.11 \mathrm{eV}$. The S4 SWNT-PEDOT:PSS-EMIM:TFSI-GuI exhibits the furthest shift with a $\Delta \mathrm{BE}$ of $0.29 \mathrm{eV}$ therefore suggesting the thiophene molecules in the film underwent the most significant bipolaron to polaron reduction. (It is also possible that the extent of the shift towards lower BE corresponds to PEDOT bonded to fewer PSS). The second most significant shift was observed for the S1 pristine PEDOT:PSS-GuI film with a $\triangle \mathrm{BE}$ of $0.19 \mathrm{eV}$ in which it was followed by the S3 PEDOT:PSS-EMIM:TFSI-GuI film 
$(\triangle \mathrm{BE} 0.12 \mathrm{eV})$. In conjunction with the UV-Vis-NIR spectra (Figure 7.5.4.2) the shift in $\mathrm{BE}$ confirms the reduction of some thiophene molecules thereby explaining the improvement in the Seebeck coefficient.

As seen in Table 7.5.4.2, some of the CX films have a ( $\Delta \mathrm{BE}$ of $0.09 \mathrm{eV})$. The UVVis-NIR spectra did not indicate reduction with ethylene glycol treatment nor did the Seebeck coefficient improve in the films. The most likely explanation for the CX films' PEDOT S $2 p$ shifts towards lower BE is due to excessive de stripping of PSS under solvo thermal stress. The ethylene glycol treatment was conducted at $160{ }^{\circ} \mathrm{C}$ for 20 minutes whereby the polar solvent was able to interact with PSS's sulphonate groups and reduce the coulombic interaction with PEDOT thereby leading to significant removal of PSS. ${ }^{126,323,329,511,512}$ This contrasts XPS quantification of the ethylene glycol treated film seen in chapter 4.8 Figure 4.8.2 under room temperature whereby only a small amount of PSS was removed from the sample. Subsequently, when PEDOT is bonded to fewer PSS molecules (containing electronegative Oxygen groups), PEDOT's S 2p electrons will require lower kinetic energy to remove an electron from the outer shell. 

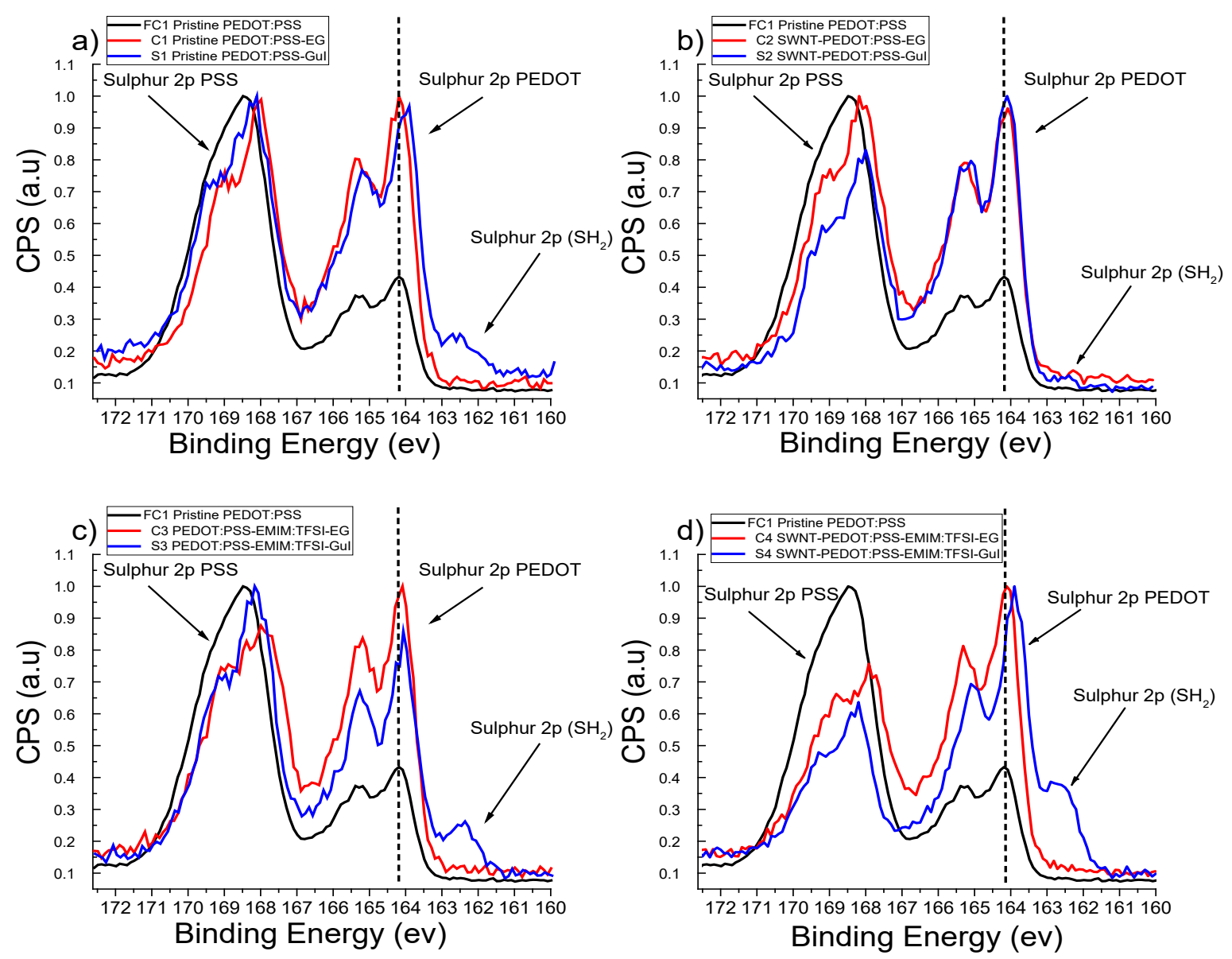

Figure 7.5.4.6 depicting the normalized, overlaid Sulphur $2 p$ spectrum for PEDOT:PSS. The region around $164 \mathrm{eV}$ corresponds to the PEDOT region meanwhile, upwards $168 \mathrm{eV}$ towards higher BE represents the PSS region. The FC1 pristine PEDOT:PSS is present as a control. 
Table 7.5.4.2 depicting the binding energy of the Sulphur 2p (PEDOT) region for the FC1 and all CX and SX films as well as the change in BE $(\triangle \mathrm{BE})$ measured in (eV) corresponding to each film relative to the FC1 pristine PEDOT:PSS.

\begin{tabular}{|l|l|l|}
\hline Film & $\begin{array}{l}\text { BE (eV) position } \\
\text { of PEDOT's S } \\
2 p_{3 / 2}\end{array}$ & $\begin{array}{l}\text { Change in BE }(\Delta \mathrm{BE}) \text { in } \\
(\mathrm{eV}) \text { relative to FC1's S } \\
2 \mathrm{p}_{3 / 2} \text { peak at (164.01) }\end{array}$ \\
\hline FC1 pristine PEDOT:PSS & 164.01 & 0.00 \\
\hline C1 pristine PEDOT:PSS-EG & 163.95 & 0.06 \\
\hline C2 SWNT-PEDOT:PSS-EG & 163.91 & 0.10 \\
\hline C3 PEDOT:PSS-EMIM:TFSI-EG & 163.92 & 0.09 \\
\hline $\begin{array}{l}\text { C4 SWNT-PEDOT:PSS } \\
\text { EMIM:TFSI-EG }\end{array}$ & 163.92 & 0.09 \\
\hline S1 pristine PEDOT:PSS-GuI & 163.82 & 0.19 \\
\hline S2 SWNT-PEDOT:PSS-GuI & 163.90 & 0.11 \\
\hline S3 PEDOT:PSS-EMIM:TFSI-GuI & 163.89 & 0.12 \\
\hline $\begin{array}{l}\text { S4 SWNT-PEDOT:PSS } \\
\text { EMIM:TFSI-GuI }\end{array}$ & 163.72 & 0.29 \\
\hline
\end{tabular}

Figure 7.5.4.7 represents the iodine $3 \mathrm{~d}_{5 / 2}$ and $3 / 2$ spin-orbital splitting with the expected $11.3 \mathrm{eV}$ separation energy. ${ }^{513-517}$ What is evident is that the $\mathrm{S} 1$ film has a small noisy signal for $\mathrm{I}_{3}^{-}$anions. This suggests that smaller quantity of $\mathrm{I}_{3}^{-}$was present in the film compared to the other films. The S3 and S2 films although showing a weak signal appear to have slightly less noisy signals. The S4 exhibited a strong clean signal. The BE for the S4 film's I $3 \mathrm{~d}_{5 / 2}$ and $3 / 2$ peak is $618.6 \mathrm{eV}$ and $630.1 \mathrm{eV}$, respectively. The BE for the $\mathrm{S} 2$ and S3 film's I $3 \mathrm{~d}_{5 / 2}$ and $3 / 2$ peak is the same as the $\mathrm{S} 4$ film within $+/-0.01 \mathrm{eV}$. In literature it is shown that the $\mathrm{I}^{-} \mathrm{BE}$ is often found $>619.1 \mathrm{eV}$ meanwhile for $\mathrm{I}_{3}{ }^{-}$is around $618.7 \mathrm{eV}$ (deviations depend on chemical environment). ${ }^{491,518,519}$ It is still possible that the peak represents $\mathrm{I}^{-}$however it's unlikely because usually $\mathrm{I}^{-}$having a signal at lower BE is usually bonded to a metal cation like coper. ${ }^{513}$ Therefore it's argued that the BE obtained in the XPS spectra suggests that the iodine signal corresponds to the $\mathrm{I}_{3}^{-}$species adsorbed on the film $185 \mid \mathrm{P}$ a g e 
surface thereby corroborating the observed UV-Vis-NIR for the SX films (Figure 7.5.4.3 (b). (Not shown), the CX films had no distinct peaks at the iodide region indicating some contamination.
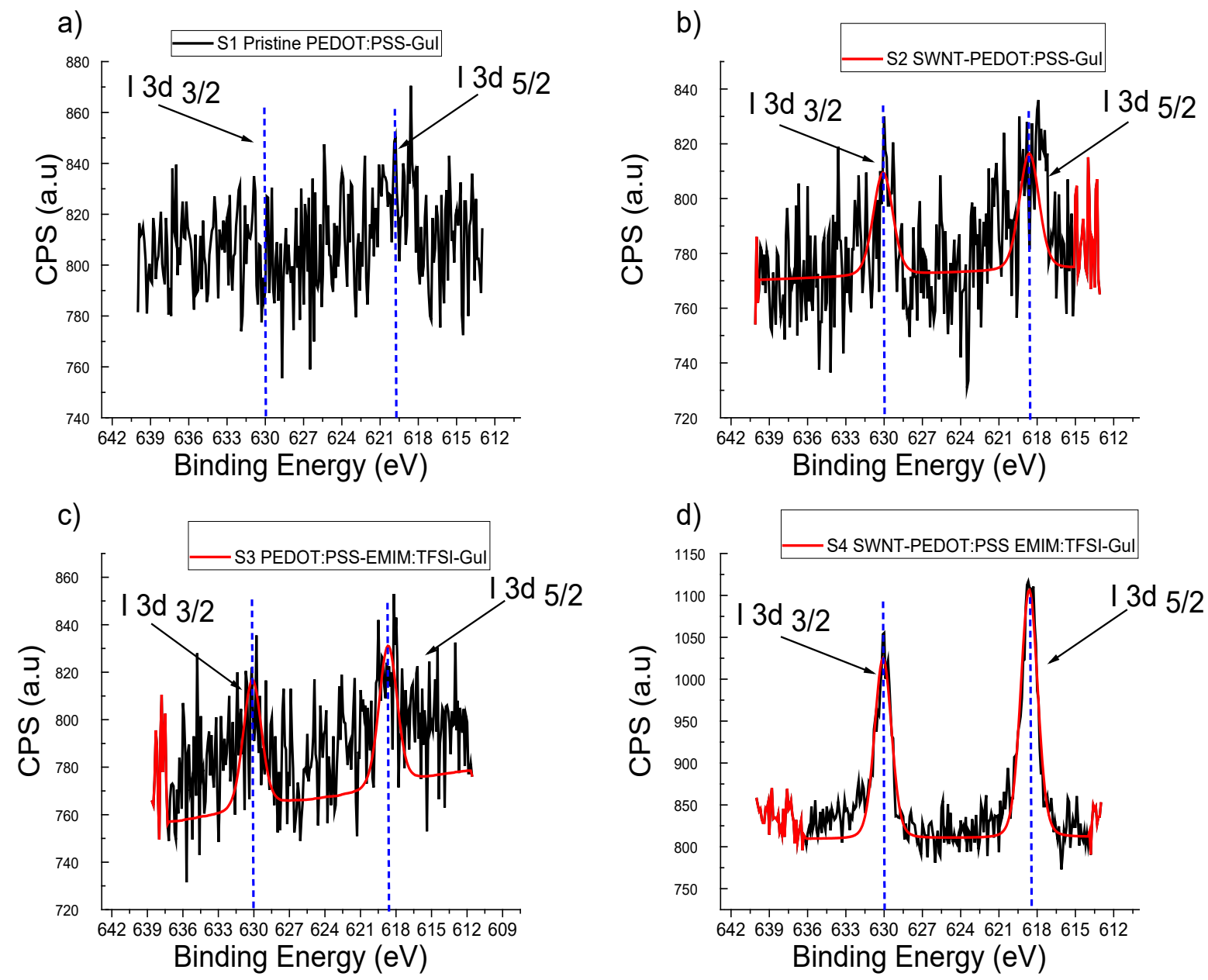

7.5.4.7 depicting the Iodine $3 d$ Spin-orbital splitting for the SX films with the 5/2 peak shown at $618.7 \mathrm{eV}$ and $3 / 2$ around $630 \mathrm{eV}$. 


\subsection{Surface characterization of PEDOT:PSS films using XPS}

\subsubsection{Analysis of Carbon 1s spectra}

XPS wide spectra were obtained for the FC1 and all the CX and SX films (not shown). There are expected elemental peaks based on known materials composition (carbon, oxygen, nitrogen, iodide, and sulphur,). For the samples with no deliberate nitrogen treatment the appearance of small nitrogen peaks $\left(\mathrm{NH}_{3}\right.$ and $\left.\mathrm{NH}_{4}^{+}\right)$can be explained by reaction between atmospheric $\left(\mathrm{NH}_{3}, \mathrm{H}_{2} \mathrm{O}\right.$ and PSS) during the heat treatment as explained by Crispin et al and corroborated by other researchers. ${ }^{196,361,520,521}$ High resolution XPS spectra were obtained for carbon, sulphur, nitrogen, and iodine where relevant for the FC1 pristine PEDOT:PSS, as well as for all CX and SX films. The spectra were charge corrected using sp ${ }^{2} \mathrm{C}$ at $284.8 \mathrm{eV}$ due to material containing nonconductive components (PSS) leading to a compensation by the XPS system. ${ }^{309,522,523}$ (Please refer to chapter 3.3 for detailed methodology).

Figure 7.6.1.1 depicts the carbon 1s spectra (C 1s) for the FC1, C1 and S1 films. As expected, the highest peak intensity was the $\mathrm{sp}^{2} \mathrm{C}$ at $284.8 \mathrm{eV}$ deriving from PEDOTs thiophene ring and contributions from the benzene ring in PSS. ${ }^{309,524,525}$ The peak at $285.6 \mathrm{eV}$ is representative of the $\mathrm{sp}^{3} \mathrm{C}$ as well as $\mathrm{C}-\mathrm{S}$ bonds in both PEDOT and PSS. ${ }^{198,309,526527}$ The peak is at the same position for all three films. The oxyethylene peak at $286.8 \mathrm{eV}$ is present in all films. ${ }^{309,527}$ The broad peak at $288.5 \mathrm{eV}$ has been shown in literature to represent carbonyl groups $(\mathrm{C}=\mathrm{O})$ and carboxylic acids $(\mathrm{O}-\mathrm{C}=\mathrm{O}) .{ }^{526,528-530}$ The region has also been linked to a $\pi-\pi^{*}$ transition PEDOT systems. ${ }^{385,389}$ It's however noted that most peak fitting seen in literature attributes the $\pi-\pi^{*}$ at slighter higher BE around $291 \mathrm{eV}$ in which the FC1 and C1 samples do not have a strong signal however a small peak can be seen on the S1 film. ${ }^{531-533}$ Therefore, it is likely majority of the contribution to the peak labelled $\left(\pi-\pi^{*}\right.$ shakeup/ O-C $\left.=\mathrm{O}\right)$ at around $288.5 \mathrm{eV}$ is from adventitious carbon contamination. ${ }^{534,535}$ 
When comparing Figure 7.6.1.1 (c) and (b) to (a) it is evident that the FC1 film (a) has a lower intensity around $286.6 \mathrm{eV}$. This is due to the presence of a new peak at 286.6 $\mathrm{eV}$ depicting the $(\mathrm{C}-\mathrm{OH})$ bond as seen for the on the $\mathrm{C} 1$ and $\mathrm{S} 1$ films. ${ }^{536-541}$ On further analysis the S1 film appears to have a more intense $(\mathrm{C}-\mathrm{OH})$ peak suggesting more residual ethylene glycol (EG). The origin of the new peak can be explained by understanding the treatment conditions. The CX and SX films were treated with ethylene glycol at $160{ }^{\circ} \mathrm{C}$ for 20 minutes. Although the films were rinsed with water it is logical for there to be residual EG on the film. It is noted that during the EG treatment on the CX films as well as the GuI solution treatment (ethylene glycol is the solvent) on the SX films that (ethylene glycol/water) vapour was observed to evaporate from surface of each film. Because the films were treated at $160{ }^{\circ} \mathrm{C}$ and the boiling point of pure ethylene glycol is $197{ }^{\circ} \mathrm{C}$ the observed evaporation must be explained. ${ }^{542}$ The PEDOT:PSS solution is around 98\% water (refer to chapter 4.4 Figure 4.4.1) therefore it is expected that during annealing for water to evaporate. This, however, only explains part of the observed evaporation because (after 10 minutes) of the $100 \mu \mathrm{L}$ ethylene glycol or GuI solution treatment on each film another $100 \mu \mathrm{L}$ of appropriate solution was placed on the surface of each film for another 10 minutes. It was observed that the second batch of the $100 \mu \mathrm{L}$ mostly evaporated. There was a thin layer of clear liquid was left on the CX films meanwhile GuI treated films exhibited a homogenously spread crystalized (off-whiteish) crystals. 
a)
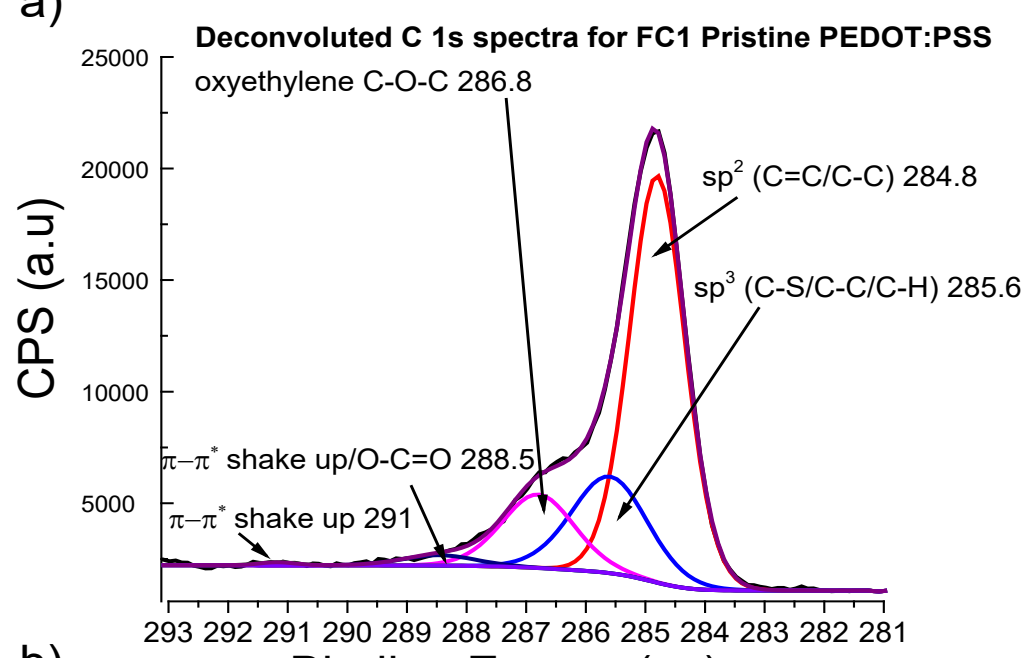

b)
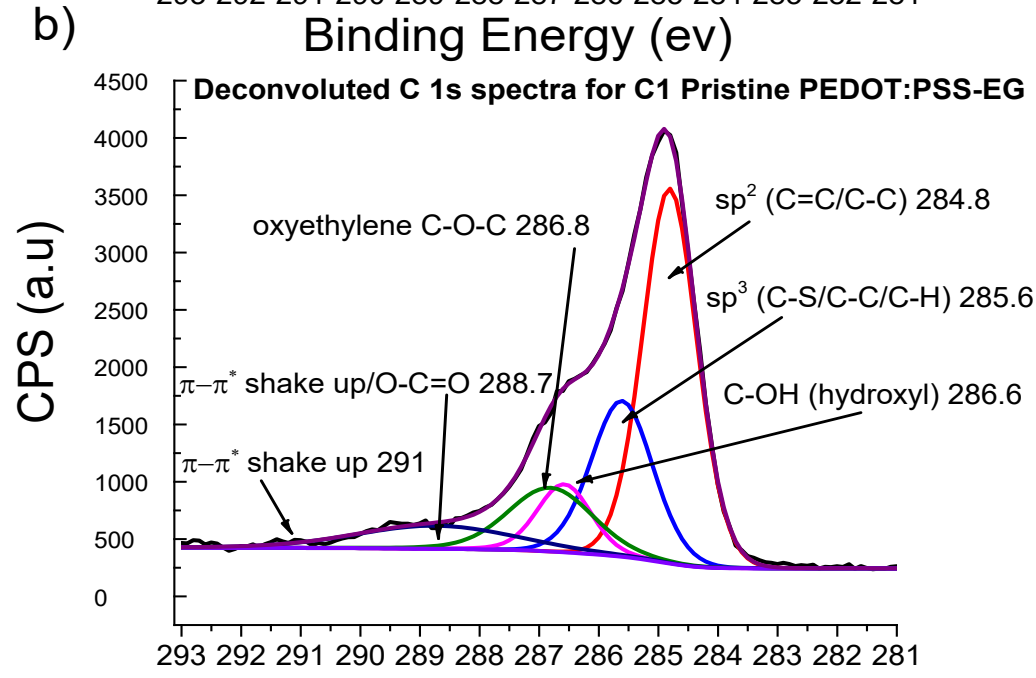

c) Binding Energy (ev)

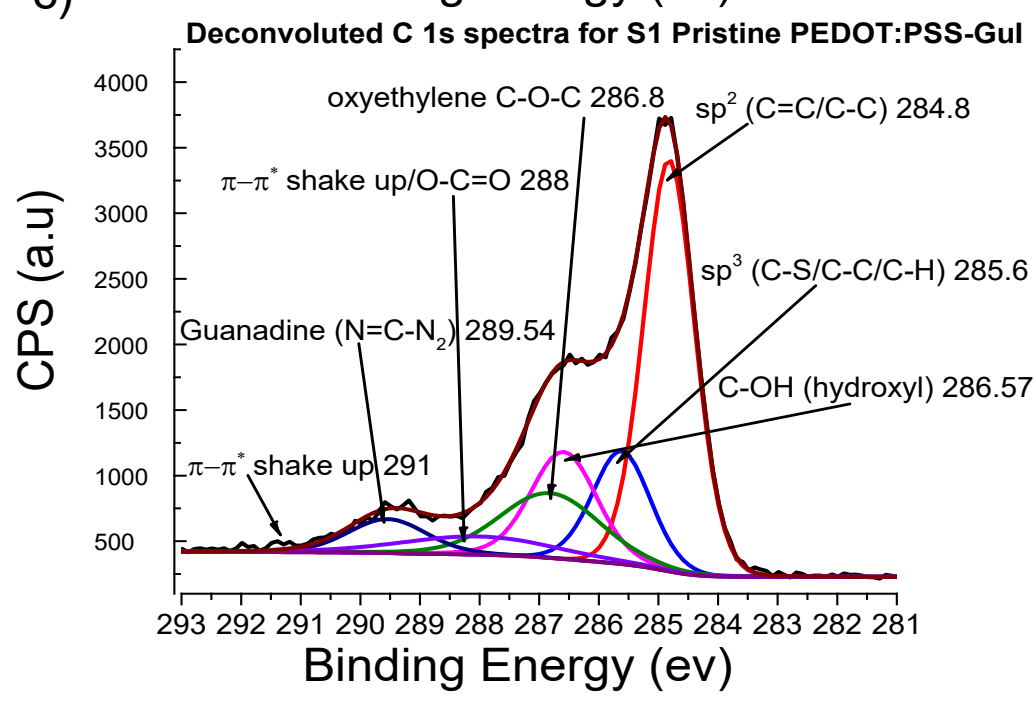


Figure 7.6.1.1 depicting the C 1s deconvoluted peaks for FC1 (a), C1 (b) and S1 films (c).

The crystals were probably guanidinium ions recrystalizing out of solution. After being washed with water several times the crystals appeared to have left the film meanwhile the clear liquid film on the CX film disappeared.

It is argued that because of the ethylene glycol being mixed with water, its vapour pressure was high enough at the surface that at $160{ }^{\circ} \mathrm{C}$ in the fume hood the solvent was able to evaporate. Literature supports the hypothesis as findings show ethylene glycol evaporation and boiling point can be affected (reduced) by lowering its mass fraction relative to water. ${ }^{543}$ In a study by Shibata et al it was reported an increased evaporation rate with decreasing ethylene glycol to water mass fraction. ${ }^{544}$ Another research study determined the relationship between mass fraction of ethylene glycol to water and it's boiling point in which it was shown even at high mass fraction $<0.8$ ethylene glycol to 0.2 water the boiling point was only $120^{\circ} \mathrm{C}$ thereby corroborating the observed evaporation at $160{ }^{\circ} \mathrm{C}$ in this study even though the boiling point of the pure solvent is $197^{\circ} \mathrm{C} .{ }^{542} \mathrm{It}$ is however noted that not "all" the solvent evaporated as all films treated with the ethylene glycol or GuI solution have the $(\mathrm{C}-\mathrm{OH})$ peak (not present in the FC1 control film).

Some of the films (C3 and S3) contain EMIM:TFSI. It was discovered in chapter 5 that the EMIM:TFSI formed a thin layer on the surface of the films it was used as a treatment thereby having XPS signals only showing TFSI $^{-}$anions and no PEDOT or PSS signals. It was also discovered that after treatment with DMSO, it was washed off the surface and PEDOT/PSS peaks were observed in the S 2 p. In Figure 7.6.1.1 there is no distinct peak at $292.4 \mathrm{eV}$ to depict the $\mathrm{CF}_{3}$ peak. ${ }^{390,545}$ This indicates that ethylene glycol and ethylene glycol GuI solution treatment also washed off the EMIM:TFSI from the surface. It is of note that the S $2 p$ peaks for PEDOT and PSS were observed for all EMIM:TFSI treated films that had the subsequent ethylene glycol, or GuI solution treatment as shown in Figure 7.5.4.6. This indicates polar solvents can wash TFSI $^{-}$off the surface of PEDOT:PSS films. There is a distinct peak emergence at $289.5 \mathrm{eV}$ regarding the $\mathrm{S} 1$ film while it cannot be seen in the $\mathrm{FC} 1$ or $\mathrm{C} 1$ films. The peak being at a higher BE indicates that it is due to contributions from the $\mathrm{N}_{2}-\mathrm{C}=\mathrm{N}$ (guanidine/guanidinium) molecule due to $\mathrm{C}$ being bonded to three electronegative $\mathrm{N}$ atoms as seen for $\mathrm{Gu}^{+}$ions in liteature. ${ }^{545-547}$

$190 \mid \mathrm{P}$ a g e 
Figure 7.6.1.2 depicts the $\mathrm{C}$ 1s spectra for the $\mathrm{C} 2$ and $\mathrm{S} 2$ films meanwhile Figure 7.6.1.3 depicts C 1s spectra for the C3 and S3 films. Further below Figure 7.6.1.4 depicts the $\mathrm{C} 1 \mathrm{~s}$ spectra for $\mathrm{C} 4$ and $\mathrm{S} 4$ film. When analysing the spectra there are no significant peak shift between samples regarding the $\left(\mathrm{sp}^{2} \mathrm{C}=\mathrm{C} / \mathrm{C}-\mathrm{C}\right)$ peak at $284.8 \mathrm{eV}, \mathrm{sp}^{3}(\mathrm{C}-\mathrm{S} / \mathrm{C}-\mathrm{C} / \mathrm{C}-\mathrm{H})$ at $285.6 \mathrm{eV}$, or the oxyethylene peak at $286.8 \mathrm{eV}$. A major difference between films however is the intensity of the peaks which indicates quantity of compound structures. Another difference between films is the emergence of the $(\mathrm{C}-\mathrm{OH})$ peak around $286.57 \mathrm{eV}$. The peak intensity is different for some samples thereby indicating different quantity of residual ethylene glycol on the surface. ${ }^{548,549}$ The peak intensity for the $\mathrm{C}-\mathrm{OH}$ bond seems randomized between samples thereby, it is not possible to induce which samples have a higher affinity at adsorbing ethylene glycol via comparisons of intensity. The $\mathrm{C} 3$ and $\mathrm{C} 4$ films have the most distinct and intense peak emergence for the $(\mathrm{C}-\mathrm{OH})$ bond at $286.57 \mathrm{eV}$. This shows the samples had the most significant residual ethylene glycol. Of note, the high-resolution $\mathrm{O}$ 1s spectrum (not shown) for the CX and SX films showed a distinct intense peak at the region for $(\mathrm{C}-\mathrm{OH})$ which was not present for the $\mathrm{FC1}$ film thereby confirming the observations seen in the carbon peaks.

As can be seen in Figure 7.6.1.2, 7.6.1.3 and 7.6.1.4 (b) at around $289 \mathrm{eV}$ there is a distinct peak present in the S2-S4 films that is not present for the $\mathrm{C} 2-\mathrm{C} 4$ films.

As proposed in relation to the S1 film (Figure 7.6.1.1) (b) the peak was credited to be the result of guanidinium/guanidine compounds adsorbed on the surface. ${ }^{545,546}$ (Please refer to Figure 7.6.2.1) showing the guanidinium oxidation states. ${ }^{485,550,551}$ The oxidation state is dependent on the $\mathrm{pH}$, temperature or any redox reactions it undergoes. ${ }^{550,552}$ The designation of the peak around $289 \mathrm{eV}$ to guanidinium/Guanidine is corroborated by the presence of the nitrogen $1 \mathrm{~s}$ peak in the range reported for guanidinium compounds (Figure 7.6.2.2). ${ }^{546}$ Licence et al conducted a study to determine the charge distribution within different guanidinium ionic liquids. ${ }^{546}$ It was determined that between 288.6-289 eV the peak for the carbon with 3 electronegative nitrogen atoms was found as was seen in this study. 


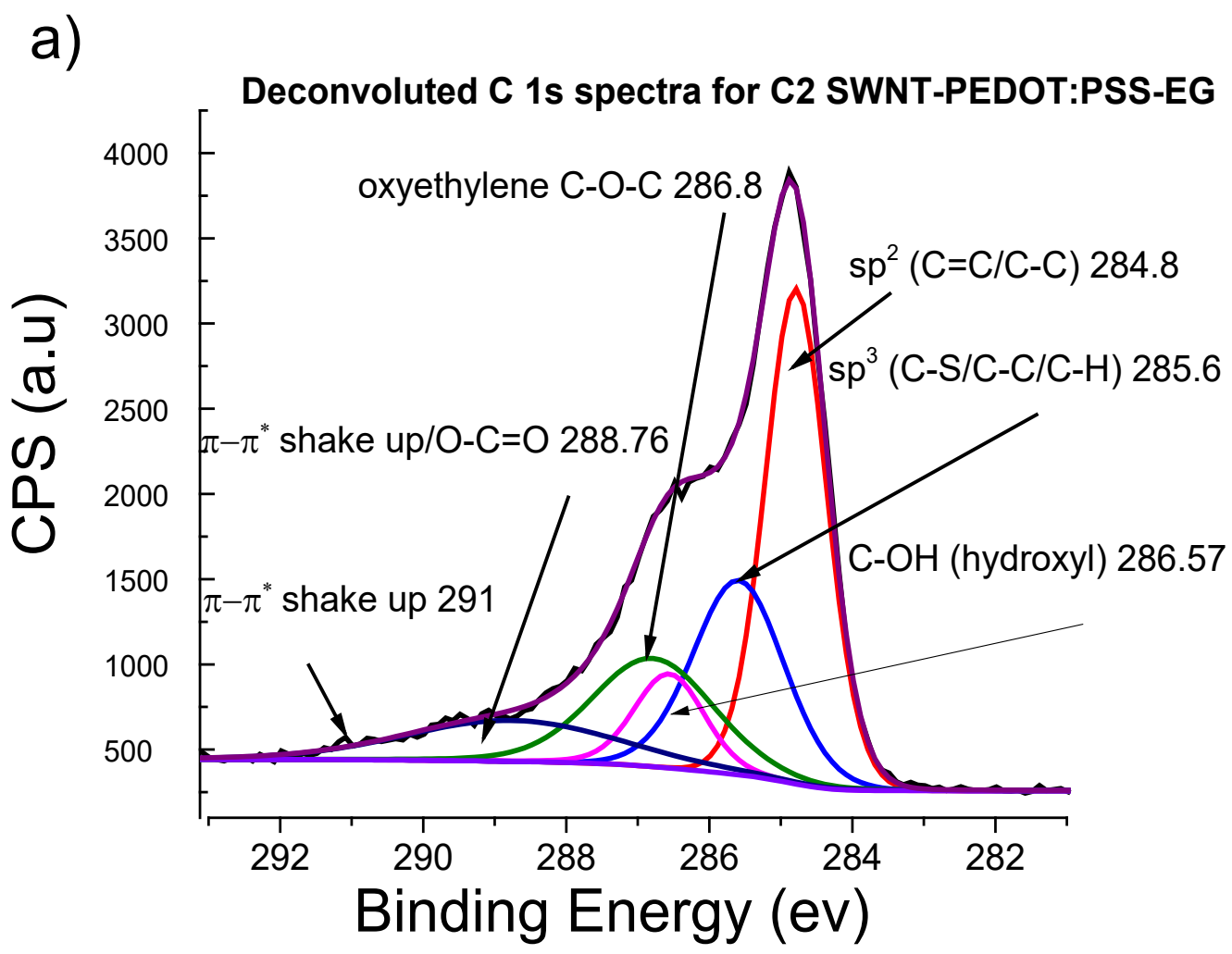

b)

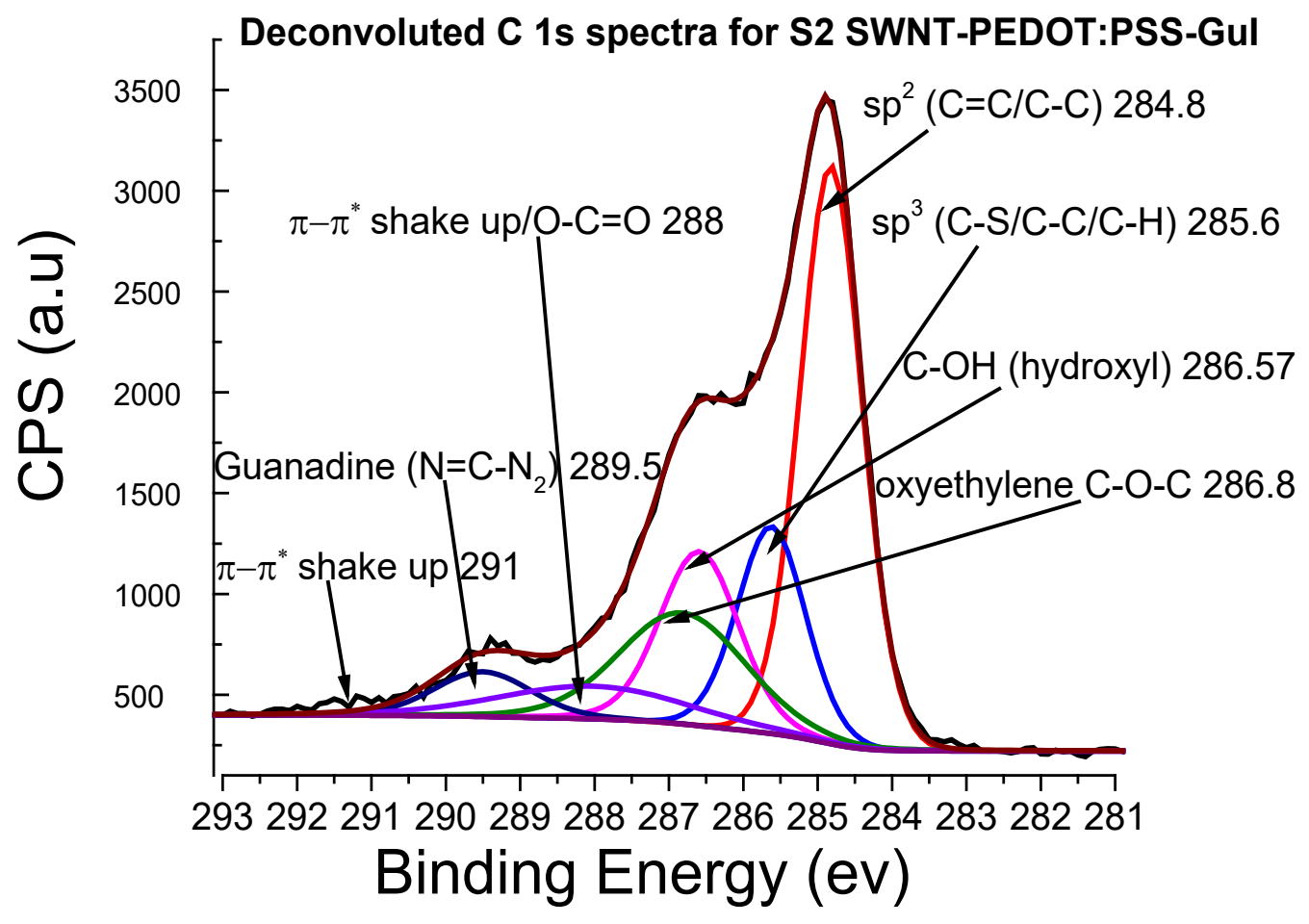

Figure 7.6.1.2 depicting the Carbon 1s spectra for the C2 (a) and S2 (b). 


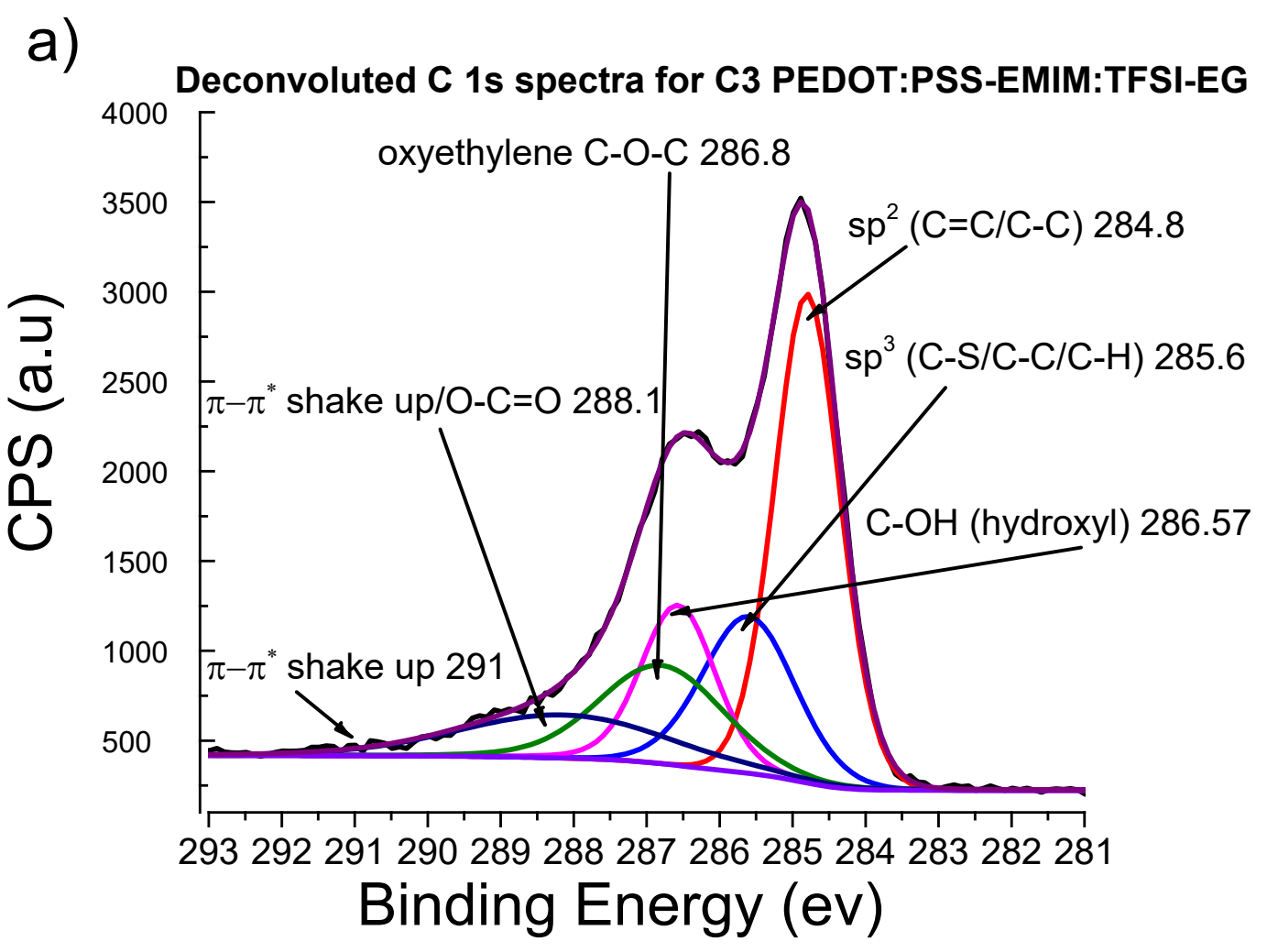

b) Deconvoluted C 1s spectra for S3 PEDOT:PSS-EMIM:TFSI-GuI

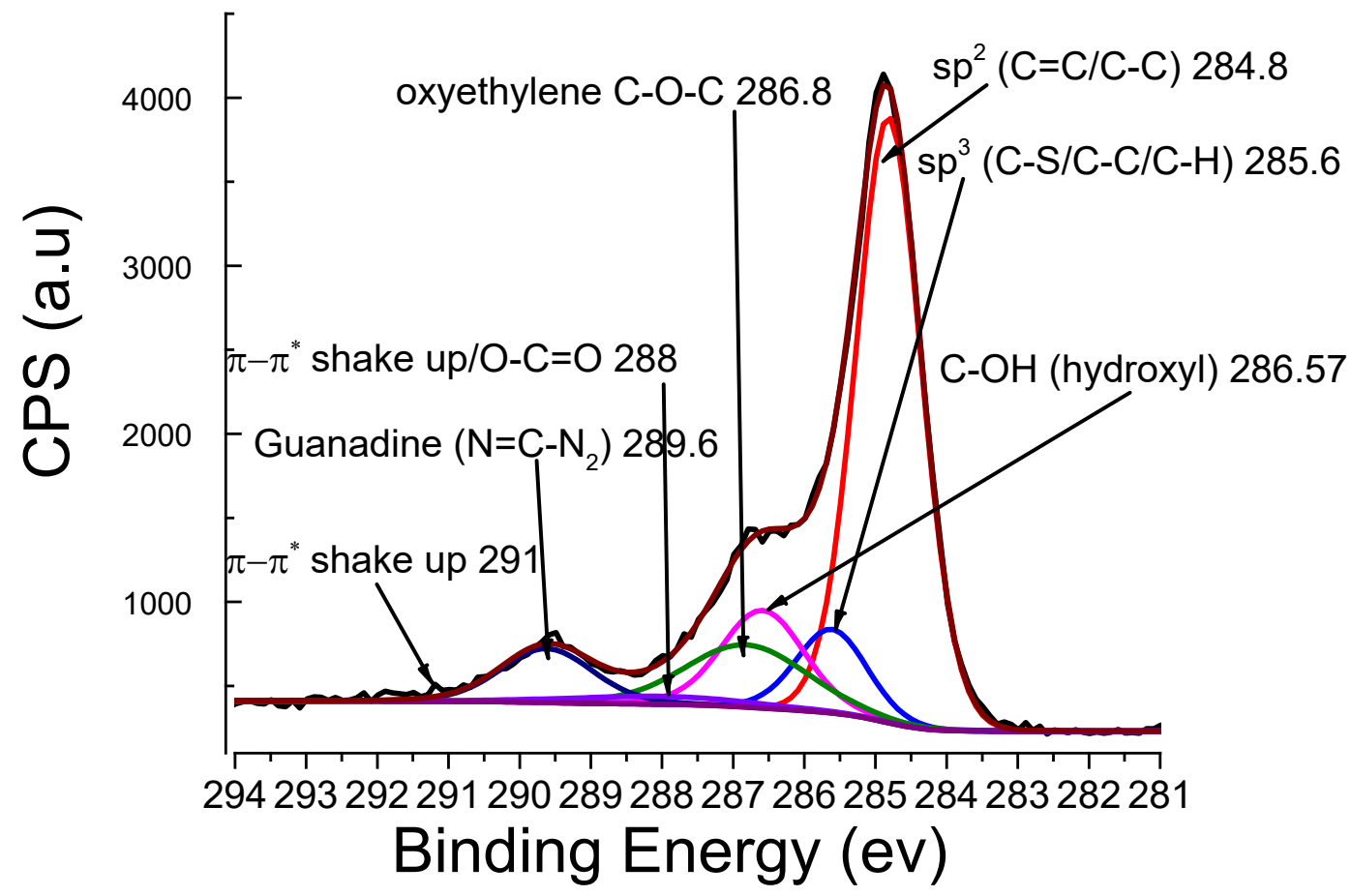

Figure 7.6.1.3 depicting the Carbon 1s spectra for the C3 (a) and S3 (b). 


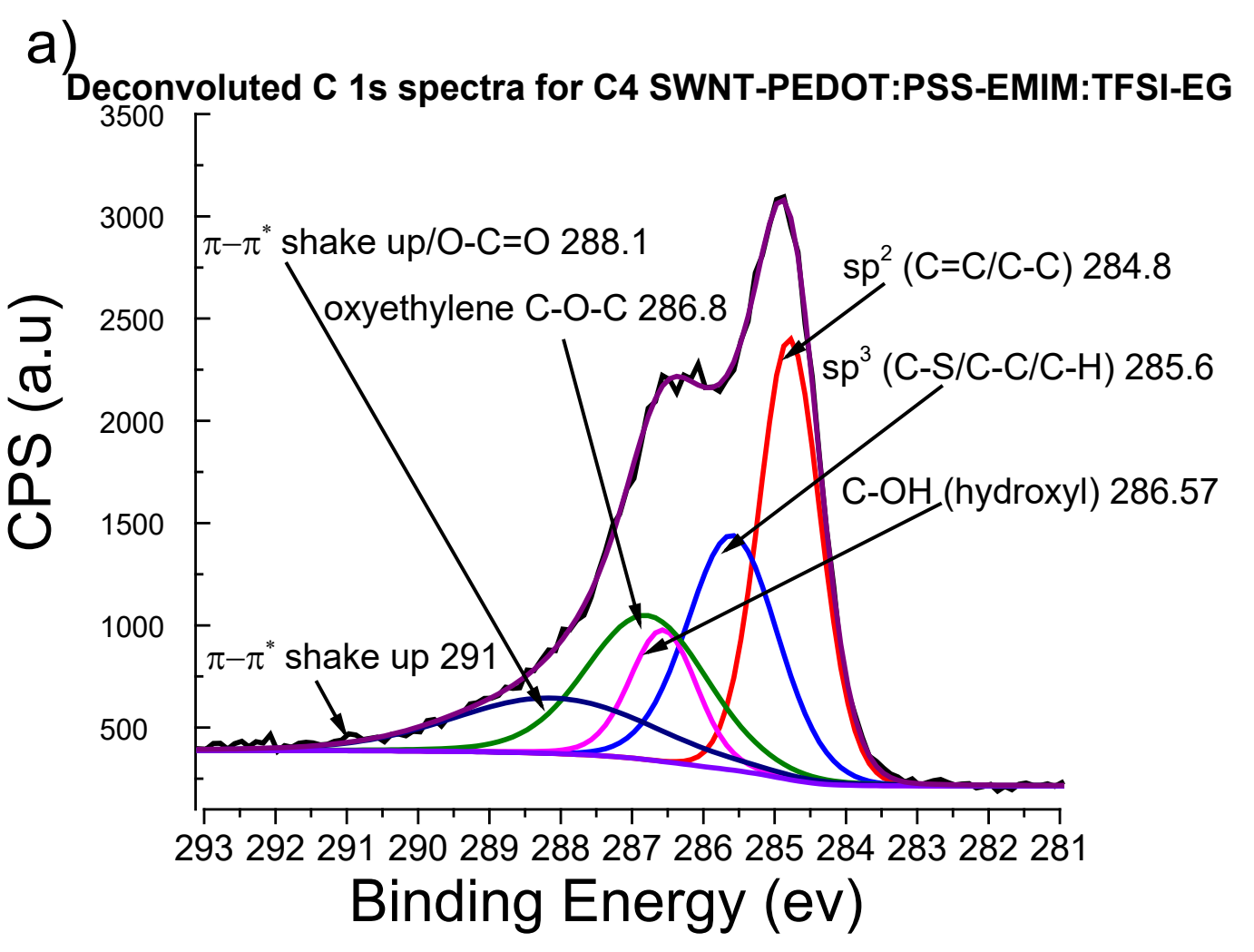

b)

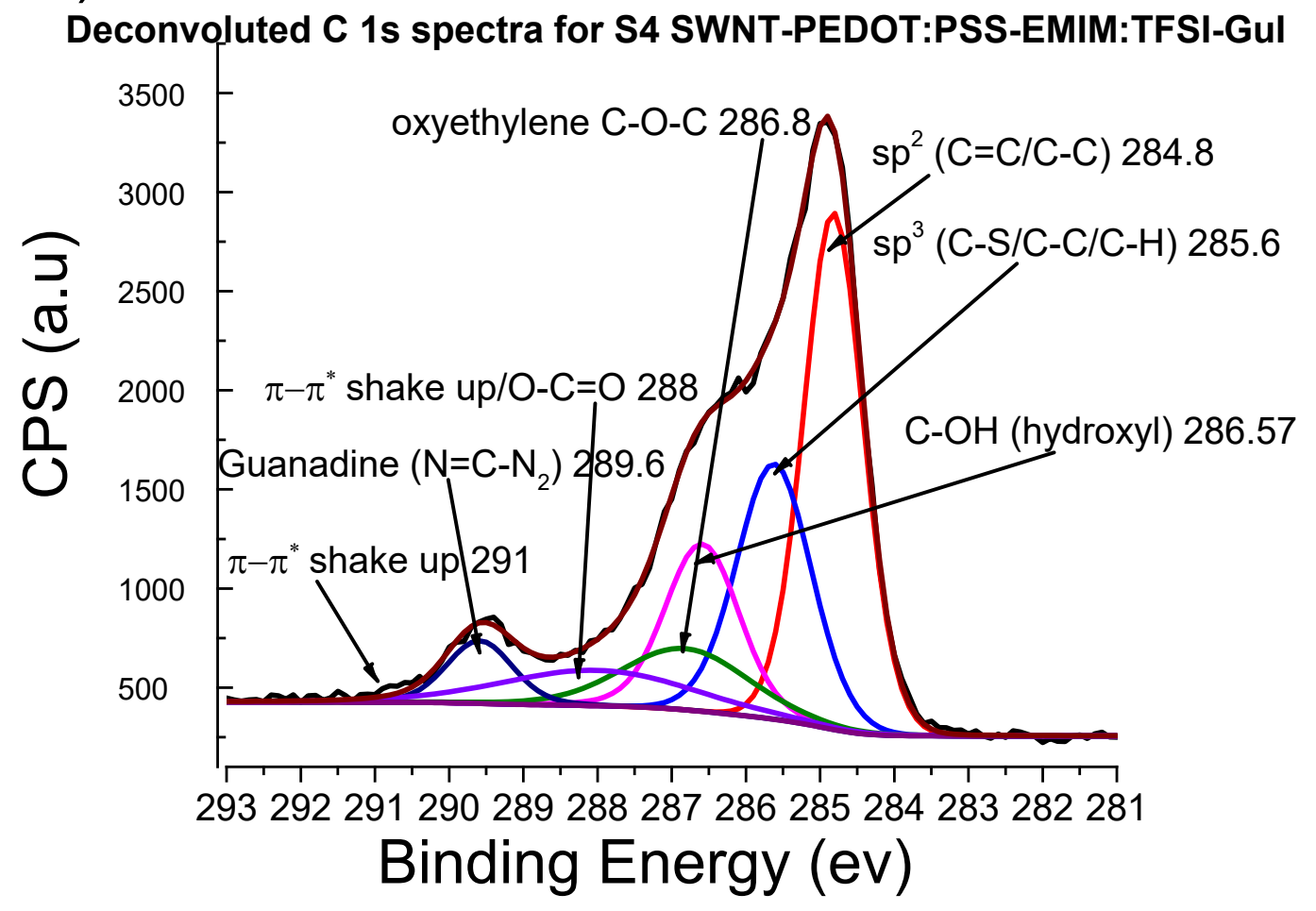

Figure 7.6.1.4 depicting the Carbon 1s spectra for the C4 (a) and S4 (b). 


\subsubsection{Analysis of $\mathbf{N} 1 \mathrm{~s}$}

Figure 7.6.2.1 depicts the guanidinium oxidation states. Figure 7.6.2.2 depicts the N 1s spectra for the FC1 and SX films. The CX films (not shown) had a significantly smaller quantity of nitrogen on the surface and the peak position was the same as for the $\mathrm{FC} 1$ film therefore indicating surface reaction between the atmospheric $\mathrm{NH}_{3}, \mathrm{H}_{2} \mathrm{O}$ and $\mathrm{PSS}^{-}$had taken place as explained by Crispin et al. ${ }^{196,385}$ As seen in Figure 7.6.2.1 are 3 different oxidation state for the guanidine. ${ }^{551}$ The guanidinium ion (a) has a positive charge on one of the nitrogen. ${ }^{553}$ In reality the charge is delocalised across the whole molecule leading to a stabilized cation. Consequently this leads to a single nitrogen environment when detected by XPS as seen in literature. ${ }^{554}$ The deprotonated molecule is the guanidine compound presented in (b) meanwhile the twice deprotonated molecule is guanidinate anion (c) in which there is also delocalisation of charge throughout the molecule. ${ }^{554}$ It would therefore be reasonable to deduce that if multiple nitrogen peaks are detected; as well as confirmed to not be resulting from $\left(\mathrm{NH}_{3}\right.$ or $\left.\mathrm{NH}_{4}^{+}\right)$then it would be evidence of deprotonation or protonation of the guanidinium ion depending on the BE. In logical sequence, from (a) to (c), guanidinium ion would exhibit the highest BE on the XPS followed by guanidine and finally guanidinate having the lowest $\mathrm{BE}$ as it is the molecule within which the $\mathrm{N} 1 \mathrm{~s}$ electron is loosely bound (assuming similar chemical environment). It is also logical to deduce that $\mathrm{NH}_{4}{ }^{+}$would have a higher $\mathrm{BE}$ still than guanidinium ions due to its charge being concentrated within only 1 nitrogen instead of being delocalized across 3 nitrogen. A study corroborates this deduction in which the $\mathrm{N} 1 \mathrm{~s}$ peak corresponding to $\mathrm{NH}_{4}{ }^{+}$was at a higher binding energy than for the guanidinium ion with a separation energy of around $2 \mathrm{eV} .{ }^{555}$ (same separation energy is seen for the $\mathrm{NH}_{3}$ and $\mathrm{NH}_{4}{ }^{+}$peak (Figure 7.6.2.2 (a).

As shown in Figure 7.6.2.2 (a) the spectrum shows the presence of $\mathrm{NH}_{3}$ species at $400.1 \mathrm{eV}$ meanwhile the oxidised $\mathrm{NH}_{4}{ }^{+}$species can be observed at $402 \mathrm{eV}{ }^{556}$ As shown for all SX films, there is an intense peak at $400.2 \mathrm{eV}$ denoted as (main $\mathrm{Gu} / \mathrm{Gu}^{+}$ peak). Except for the S2 film's N 1s spectrum, S1, S3 and S4 spectra show another peak at $398 \mathrm{eV}$ labelled (sign of deprotonation). It is not possible to be certain of 
whether the peak at $400.2 \mathrm{eV}$ is guanidinium or guanidine (Figure 7.6.2.2) due to no baseline determined in this study for GuI salt (due to unforeseen Covid-19 circumstances).

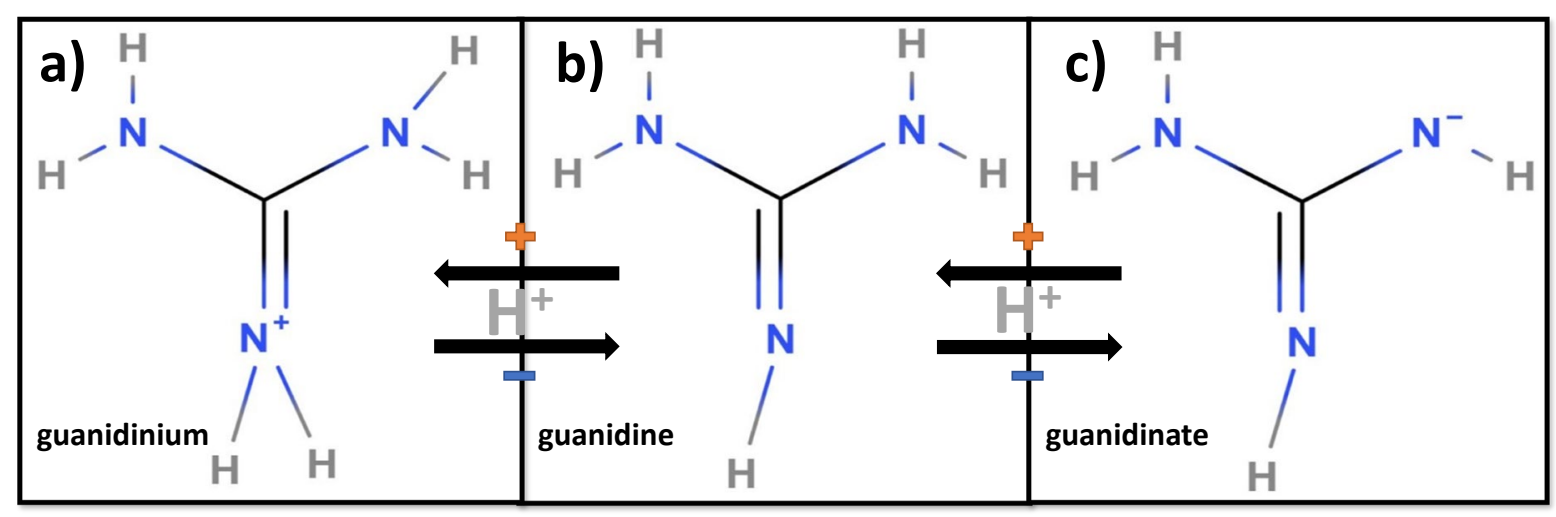

Figure 7.6.2.1 depicting the various oxidation states of Guanidine in the ethylene glycol solution. (a) Depicts the guanidinium cation suspected to be the most common form due to its ionic interaction with Iodide in $\left(\mathrm{CH}_{6} \mathrm{IN}_{3}\right)$, meanwhile (b) depicting a deprotonated neutral Guanidine, and (c) represents the deprotonated Guanidinate anion. $^{486,550}$

However, it is possible to make inferences based on bonding chemistry and BE from literature reports to indicate the oxidation state the peaks represent. In literature the guanidinium peak has been shown at around $400.5 \mathrm{eV}$ but can be higher (depending on chemical environment). ${ }^{557}$ It's known that guanidinium ion is more stable than guanidine of which is a strong base therefore it would be unlikely for there to be a significant amount of guanidine relative to guanidinium left on the film. ${ }^{552,553}$ Further still for an interpretation of the XPS spectrum presented in Figure 7.5.4.6 whereby a "new" peak was presented at $162.5 \mathrm{eV}$ labelled $\mathrm{SH}_{2}$ it is only needed to be confirmed guanidinium ions were involved in a redox reaction whereby some ions give off a $\mathrm{H}^{+}$ to catalyse a proposed desulphurization (desulphonation) in literature when referring specifically to the extraction of the sulfone group in $\operatorname{PSS}^{196}$ and the subsequent reduction via the basic guanidine species into $\mathrm{SH}_{2}$. The peak at $398 \mathrm{eV}$ provides evidence that some of the $\mathrm{N}$ in guanidinium ions in the S1, S3, and S4 films lost at least $1 \mathrm{H}^{+}$but due to the low BE indicates it suspected as guanidinate. ${ }^{558}$

In relation to the peak labelled "suspected $\mathrm{Gu}^{+}$ionically bonded $(401.2 \mathrm{eV})$ present in the S2, S3 and S4 films, it's unlikely for it to represent the $\mathrm{NH}_{4}{ }^{+}$compound (402 eV) because it's at a slightly lower BE. It is possible that the peak corresponds to 
guanidinium ion that has formed ionic interaction with $\mathrm{PSS}^{-}$or hydrogen bonding with either PEDOT's Oxyethylene or through cation $-\pi$ interactions with the aromatic rings. ${ }^{546,559}$ Due to the peak being at a slightly higher BE $(401.2 \mathrm{eV})$ than the main $\mathrm{Gu} / \mathrm{Gu}^{+}$peak $\left(400.2 \mathrm{eV}\right.$ ) it's probably between either $\mathrm{Gu}^{+} \mathrm{PSS}^{-}$ionic interaction or $\mathrm{Gu}^{+}$ $\pi$ interaction than the hydrogen bonding with Oxyethylene; because it's been reported in literature that cation $-\pi$ interactions tend to increase the binding energy. ${ }^{557,560-563}$ The reason why it's also possible for it to be representative of an ionic interaction with some $\mathrm{PSS}^{-}$molecules is because the $\mathrm{O}^{-}$atom in the sulfonate group is electronegative thereby would leading to a dipole moment away from the $\mathrm{Gu}^{+}$resulting in a higher $\mathrm{BE}$. Regarding the peak labelled suspected $\mathrm{NH}_{4}{ }^{+}$on the $\mathrm{S} 1$ film (Figure 7.6.2.2 (b) it appears accurate because it is around $402 \mathrm{eV}$ same as for the FC1 film and literature. ${ }^{556}$ The overall $\mathrm{N}$ 1s peak intensity was also small therefore showing that guanidinium is present in small quantities for the S1 film. 
a) Deconvoluted $\mathrm{N}$ 1s spectrum for FC1 Pristine PEDOT:PSS

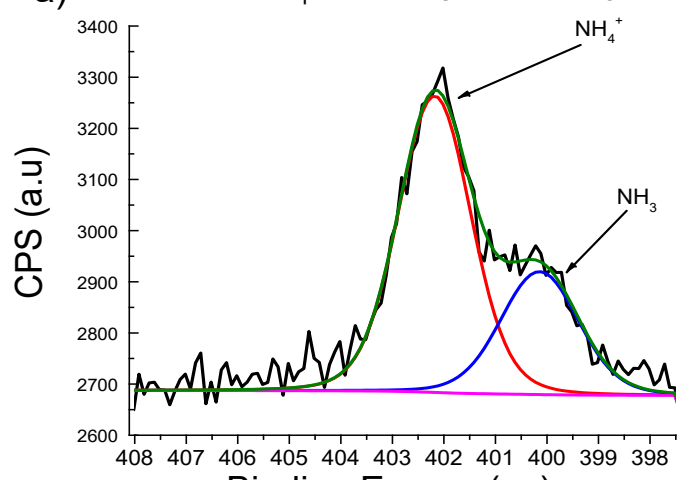

Binding Energy (ev)
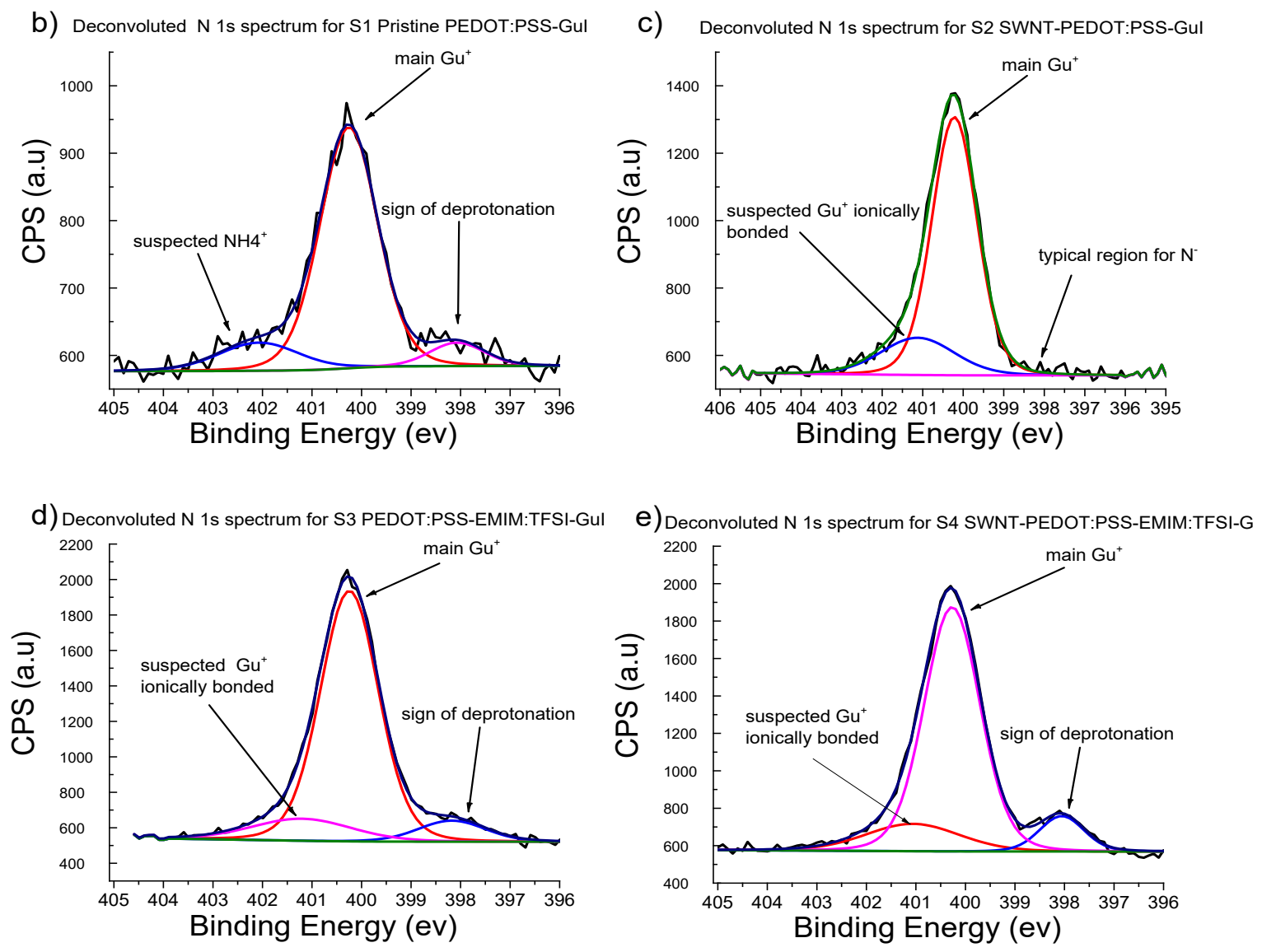

Figure 7.6.2.2 depicts the N 1s spectra for the FC1 and SX films (a) FC1, (b) S1, (c), $S 2$, (d) $S 3$, (e) $S 4$

\subsubsection{XPS quantification and desulphurization mechanism}

Figure 7.6.3.1 depicts the peak fitted S 2p spectra for the FC1 CX and SX films, meanwhile Table 7.6.3.1 the peak positions and detailed quantification data for the $S$ $2 \mathrm{p}_{3 / 2}$ and $1 / 2$ Spin-orbital splitting for the films with BEs within values reported in 
literature. ${ }^{196,309,524,564}$ As discussed in earlier experimental chapters, it is possible to detect the ratio between PEDOT and PSS on the surface of a film via XPS. ${ }^{146,279,365,565}$ If the amount of PSS relative to PEDOT is reduced (depending on the amount) an increased in electrical conductivity is usually observed. ${ }^{361,566-568}$ When significant amount of the insulating PSS is removed from the film the PEDOT core is able to reorient itself from a coiled coil shell to a more expanded coil and linear structure whereby the $\pi-\pi$ stacking distance is reduced thereby leading to stronger $\pi-\pi$ interactions and improved carrier mobility (Please refer to chapter 4.6 Figure 4.6.2). ${ }^{71,361,569-572}$

As seen in Figure 7.6.3.1 due to some films having $\mathrm{SH}_{2}\left(\mathrm{~S} \mathrm{2} \mathrm{p}_{3 / 2}\right.$ at $\left.162.5 \mathrm{eV}\right)$ instead of comparing amount of PEDOT increased in the film the reader is advised to compare the amount of PSS reduced. When looking at the PEDOT and PSS regions as shown in Figure 7.6.3.1 (a) for the FC1 film it appears that the concentration of PSS should be around $66 \%$ and PEDOT 33\%. However as seen in the detailed quantification (Table 7.6.3.1) concentration of PSS in the FC1 film is $55.92 \%$. As can be seen in the S $2 p$ spectra for all films in Figure 7.6.3.1, PEDOT's S 2 $p_{3 / 2}$ and ${ }_{1 / 2}$ Spinorbital splitting have an asymmetrical tail tending towards higher BEs due to some of the thiophenes being in the $\mathrm{PEDOT}^{+}$and $\mathrm{PEDOT}^{2+}$ oxidation states. ${ }^{573-575}$ Therefore when integrating the area under the peaks the $55.92 \%$ concentration of PSS is explained.

In reference to the electrical conductivity of the CX films (Figure 7.5.2.1 (a); when comparing the amount of PSS in each CX film as shown in Table 7.6.3.1 and Figure 7.6.3.1, it is evident that films with the poorest conductivity $(\mathrm{C} 1$ and $\mathrm{C} 2)$ also had the highest PSS concentration in the films (28.9\% and 32.96\%) respectively. Still both the $\mathrm{C} 1$ and $\mathrm{C} 2$ films had considerably higher conductivity than the FC1 film in which the PSS concentration is higher still (55.91\%). Furthermore, the C3 and C4 films had much higher conductivities and much smaller PSS concentration $(23.27 \%$ and $19.29 \%$ ) respectively. It's argued therefore that PSS removal and the subsequent reorientation of PEDOT chains from coiled coil structures to more linear $\pi-\pi$ stacking improved carrier mobility as indicated via the red shift in the Raman $C_{\alpha}=C_{\beta}$ 
symmetrical stretching (chapter 7.5.3) were significant contributing factors leading to the improved conductivity observed in the CX films relative to $\mathrm{FC} 1 .{ }^{126,298,361,378}$ For the SX films the S4 film has the lowest PSS concentration (23.82\%) which is like the C4 film's (19.29\%). Unlike what was observed for the CX films however, there is a break in the pattern in which the more conductive films had less PSS. This is because the S3 film was second most conductive SX film (Figure 7.5.2.1 (b) but had a higher relative PSS concentration of $33.86 \%$ compared to the S1 and S2 had $(31.83 \%$ and $26.4 \%$ ) respectively. It is off note that the concentration of PSS in the S3 film was still significantly lower than FC1 film (55.92\%). When comparing the conductivity of the $\mathrm{C} 4\left(2240 \mathrm{~S} \mathrm{~cm}^{-1}\right)$ and $\mathrm{S} 4$ film $\left(3665 \mathrm{~S} \mathrm{~cm}^{-1}\right)$ at $40^{\circ} \mathrm{C}$ the $\mathrm{S} 4$ film's conductivity is almost twice as high. To explain this phenomenon, it is important to note that both films had similar PSS removed from the film however the S4 film had a treatment in which charge carriers were reduced (bipolaron to polaron). Logically the reduced carrier concentration should have led to reduced electrical conductivity however as seen for the S4 and other SX films the conductivity was improved for each film relative to its $\mathrm{CX}$ counterpart.

To explain this, it is possible that the SX films may have had significantly higher carrier mobility (Please refer to chapter 7.2 Equation 3). The improved carrier mobility may then off-set the reduced charge carriers observed in the UV-Vis-NIR spectra (Figure 7.5.4.2) for SX films. (Please note UV-Vis-NIR only indicates reduced carriers along the thiophene backbone (bipolarons/polarons). This hypothesis can be confirmed if XRD measurements are conducted and diffraction peaks are compared to known regions depicting crystallinity and mapping it to stacking orientation of films. ${ }^{361}$ It was not possible however to get meaningful diffraction peaks. Another possible explanation and one which seems more likely is that because GuI treatment contained ionic charge carriers, a considerable amount of $\mathrm{Gu}^{+}$was adsorbed on the film surface of the SX films as confirmed by the SX film's C 1s and N 1s spectra. It is known that the charge is delocalised across the $\mathrm{Gu}^{+}$ion therefore it is possible that the ions form some cation- $\pi$ interactions (guanidinium is known to make) $551,559,560$ with PEDOT and improve bulk carrier concentration. This would be able to be proven via Hall effects measurements in future studies. 
When analysing Figure 7.6.3.1 there is a "new" S 2 $p_{3 / 2}$ signal at around $162.5 \mathrm{eV}$ present in the S1, S3 and S4 films. The peak is not present in the FC1 or CX films. To identity the origin of the peak a few logical considerations must be explored and challenged by the evidence. The peak is representative of a sulphide whereby sulphur such as $\mathrm{SH}_{2}$ is in a $2^{-}$oxidation state as seen in literature. ${ }^{307,504,508-510}$ The initial thought was that the signal was due to "very" reduced thiophene chains. This is because as confirmed by UV-Vis-NIR, the $\mathrm{I}^{-}$reduced some thiophene molecules. This is a weak argument however because the thiophene was shown to only be reduced from bipolarons to some polarons as confirmed by lack of emergence of a peak at the (neutral/ $\pi-\pi^{*}$ ) region $600 \mathrm{~nm}$ ). If the peak at $162.5 \mathrm{eV}$ represented PEDOT reduced to a negative state it would have had to have been reduced first to the neutral state. Another consideration would be that the peak is evidence of "degraded" PEDOT. PEDOT chains that have had some thiophene rings opened and reduced. If this were the case, then the SX films that exhibited the peak would have had considerably lower electrical conductivity owning that PEDOT is the conductive component in the PEDOT:PSS films. It would also have been evident of lower PEDOT concentration in the SX films. This of course was not the case. The argument that the peak is $\mathrm{SH}_{2}$ is on based on eliminating the prior arguments which would leave the option that the peak originated from degraded PSS under a known process of desulphonation that can take place under thermal and acidic conditions. ${ }^{196,576}$

In literature it is often argued with some evidence that a desulphonation of PSS sometimes occurs due to a reaction between some atmospheric $\mathrm{NH}_{3}$ and PSS in presence of acidic catalyst such as $\mathrm{H}_{3} \mathrm{O}^{+}$under some thermal conditions in PEDOT:PSS films leading to the extraction of $\mathrm{SO}_{3}$ that then reacts with $\mathrm{H}_{2} \mathrm{O}$ to produce some $\mathrm{H}_{2} \mathrm{SO}_{4} / \mathrm{HSO}_{4}^{-}$. (Please see a study by Crispin et al for full balanced mechanism) $)^{196}$ and the work by other researchers for evidenced desulphurization of sulfonated compounds as well as specifically desulphonation of benzenesulfonic compounds. ${ }^{577-579}$ In some studies ionic liquids such as $N$-butyl pyridinium dicyanamide $\quad\left(\left[\mathrm{C}_{4} \mathrm{Py}\right]\left[\mathrm{N}(\mathrm{CN})_{2}, \quad\right.\right.$ and $\quad N$-butylpyridinium bis(trifluoromethylsulfonyl)imide $\left(\left[\mathrm{C}_{4} \mathrm{Py}\right]\left[\mathrm{NTf}_{2}\right]\right)$ have to been used to effectively remove aromatic sulphur groups such as benzothiophenes from various samples 
through desulphurization, ${ }^{576,580}$ or in ethylene glycol/derivates (in presence of ionic liquid) mediated desulphurization processes. ${ }^{578}$

$202 \mid \mathrm{P}$ a g e 


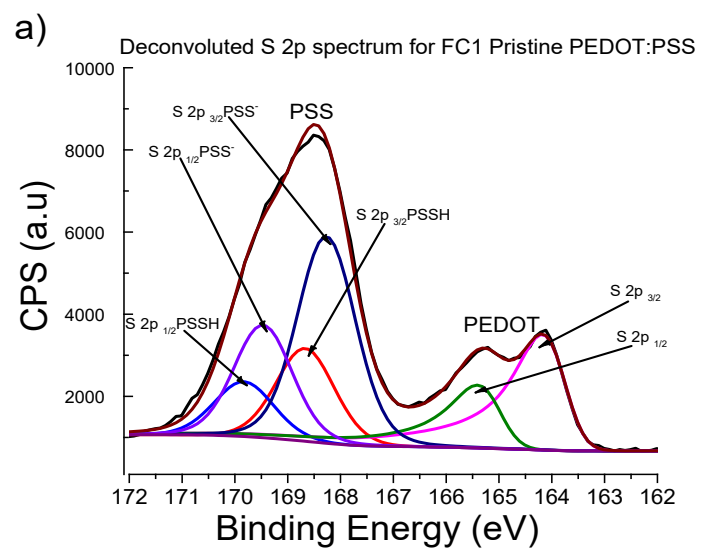

b)

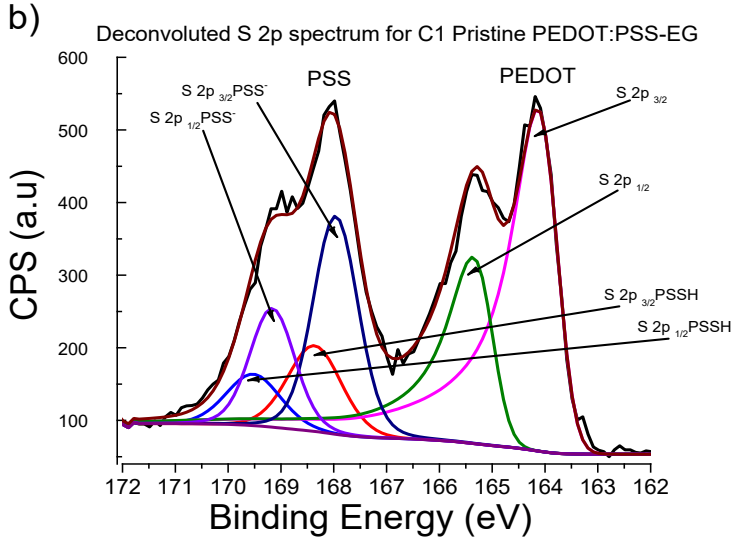

d) Deconvoluted S 2p spectrum for C2 SWNT-PEDOT:PSS-EG

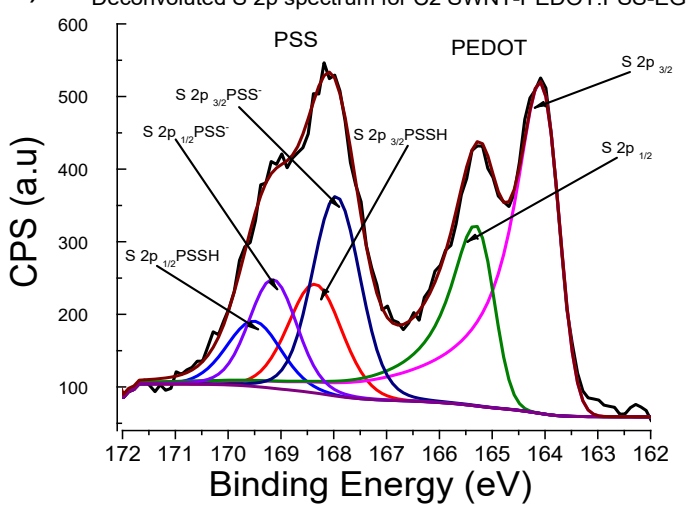

c)

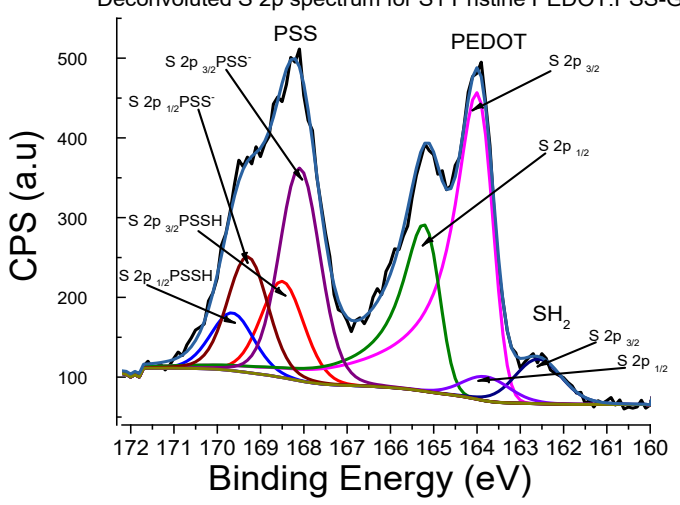

e) Deconvoluted S $2 p$ spectrum for S2 SWNT-PEDOT:PSS-Gul

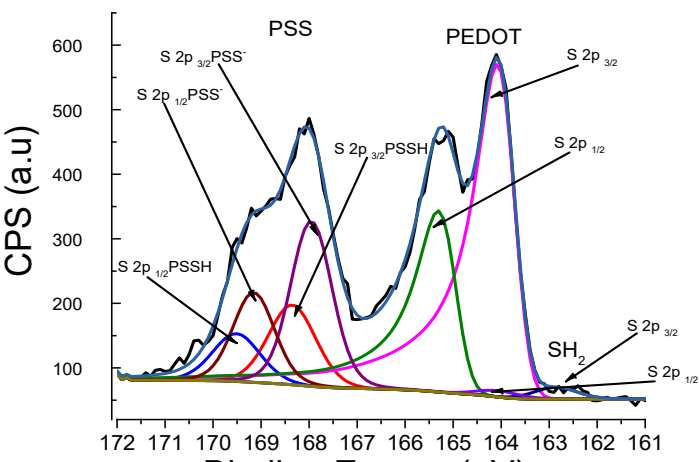

Binding Energy (eV) 


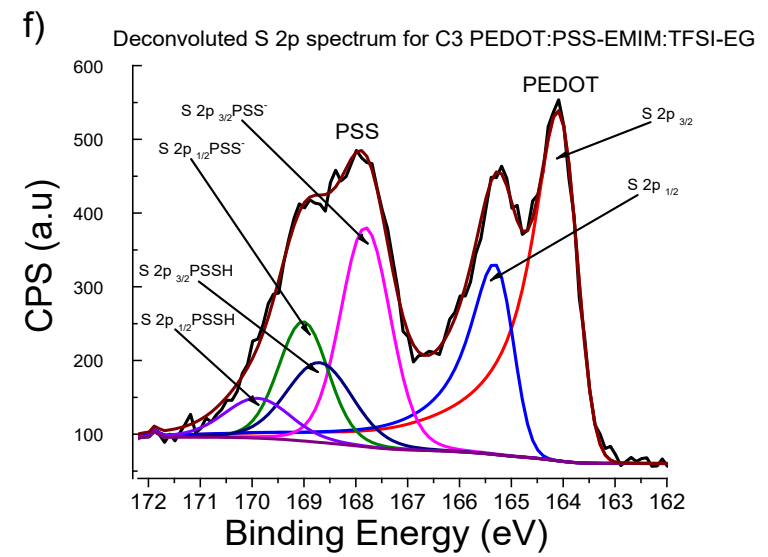

h) Deconvoluted S 2p spectrum for C4 SWNT-PEDOT:PSS-EMIM:TFSI-EG

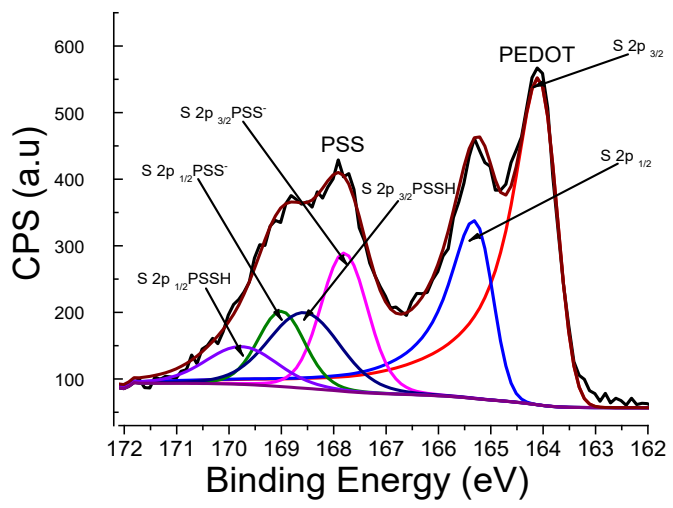

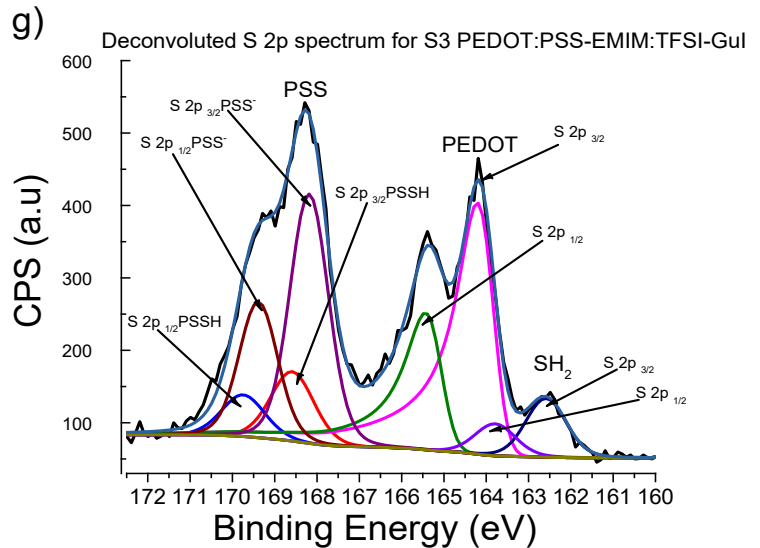

i) Deconvoluted S $2 p$ spectrum for S4 SWNT-PEDOT:PSS-EMIM:TFSI-Gul

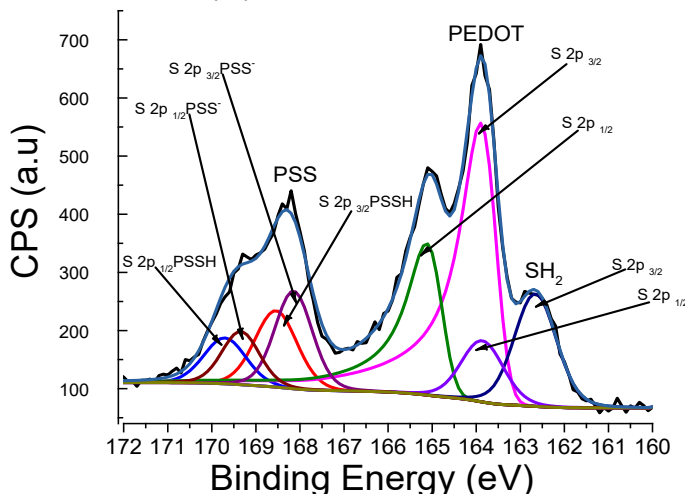

Figure 7.6.3.1, depicting the high-resolution peak fitted Sulphur $2 p$ Spin-orbital splitting (3/2)/ and (1/2). For PEDOT, the (3/2) split is centred around $164 \mathrm{eV}$ while the (1/2) splitting is around $165 \mathrm{eV}$. PSS can be shown to have two distinct splitting doublets due to the PSS's sulfonate compounds being in two distinct chemical environment in which the (3/2) split for the PSS is centred around $168 \mathrm{eV}$ while the corresponding (1/2) splitting is around $169.5 \mathrm{eV}$. The PSSH (3/2) split can be found at around $168.6 \mathrm{eV}$ meanwhile the (1/2) split can be found at around $169.8 \mathrm{eV}$. The peak at the lowest BE is depicting the $\mathrm{SH}_{2}$ peak with the (3/2) and (1/2) at around 162.6 and a $162.8 \mathrm{eV}$ respectively. (a) depicts the FC1 pristine PEDOT:PSS, meanwhile $(b, d, f, h)$ depicts the CX films and (c, e, g, i) depict the SX films as labelled. 
Table 7.6.3.1 depicting the detailed quantification data for the FC1 and all CX and SX films

\begin{tabular}{|c|c|c|c|c|c|c|}
\hline Film & Name & $\begin{array}{l}\text { Peak } \\
\text { Position } \\
\text { BE (eV) }\end{array}$ & $\begin{array}{l}\mathrm{S} \\
\% \text { conc }\end{array}$ & $\begin{array}{l}\text { Total } \\
\% \text { conc } \\
\text { PSS }\end{array}$ & \begin{tabular}{|l|} 
Total \\
$\%$ conc \\
PEDOT
\end{tabular} & $\begin{array}{l}\text { Total } \\
\% \mathrm{SH}_{2} \\
\text { conc }\end{array}$ \\
\hline \multirow[t]{6}{*}{ FC1 pristine PEDOT:PSS } & $\mathrm{S} 2 \mathrm{p}_{3 / 2} \mathrm{PSSH}$ & 168.67 & 18.62 & 55.92 & 44.08 & 0 \\
\hline & $\mathrm{S} 2 \mathrm{p}_{1 / 2} \mathrm{PSSH}$ & 169.83 & 11.17 & & & \\
\hline & $\begin{array}{l}\mathrm{S} \\
\text { PEDOT }\end{array}$ & 164 & 28.54 & & & \\
\hline & $\begin{array}{l}\mathrm{S}^{2 \mathrm{p}_{1 / 2}} \\
\text { PEDOT }\end{array}$ & 165.2 & 15.54 & & & \\
\hline & $\mathrm{S} 2 \mathrm{p}_{3 / 2}$ PSS- & 168.27 & 16.92 & & & \\
\hline & $\mathrm{S} 2 \mathrm{p}_{1 / 2}$ PSS- & 169.47 & 9.21 & & & \\
\hline \multirow[t]{6}{*}{ C1 pristine PEDOT:PSS-EG } & $\mathrm{S} 2 \mathrm{p}_{3 / 2} \mathrm{PSSH}$ & 168.36 & 9.85 & 28.9 & 71.1 & 0 \\
\hline & $\mathrm{S} 2 \mathrm{p}_{1 / 2} \mathrm{PSSH}$ & 169.52 & 5.91 & & & \\
\hline & $\begin{array}{ll}\mathrm{S}^{2} & 2 \mathrm{p}_{3 / 2} \\
\text { PEDOT }\end{array}$ & 163.95 & 46.05 & & & \\
\hline & $\begin{array}{l}\mathrm{S} \\
\text { PEDOT }\end{array}$ & 165.18 & 25.05 & & & \\
\hline & S 2p p $p_{3 / 2}$ PSS- & 167.96 & 8.51 & & & \\
\hline & $\mathrm{S} 2 \mathrm{p}_{1 / 2}$ PSS- & 169.16 & 4.63 & & & \\
\hline \multirow[t]{5}{*}{ C2 SWNT-PEDOT:PSS-EG } & $\mathrm{S} 2 \mathrm{p}_{3 / 2} \mathrm{PSSH}$ & 168.35 & 12.55 & 32.96 & 67.03 & 0 \\
\hline & $\mathrm{S} 2 \mathrm{p}_{1 / 2} \mathrm{PSSH}$ & 169.51 & 7.52 & & & \\
\hline & $\begin{array}{l}\mathrm{S} \\
\text { PEDOT }\end{array}$ & 163.91 & 43.41 & & & \\
\hline & $\begin{array}{l}\mathrm{S} \\
\text { PEDOT }\end{array}$ & 165.14 & 23.62 & & & \\
\hline & S 2p p $3 / 2$ PSS- & 167.95 & 8.35 & & & \\
\hline
\end{tabular}




\begin{tabular}{|c|c|c|c|c|c|c|}
\hline & $\mathrm{S} 2 \mathrm{p}_{1 / 2}$ PSS- & 169.15 & 4.54 & & & \\
\hline C3 PEDOT:PSS-EMIM:TFSI-EG & $\begin{array}{l}\mathrm{S} \quad 2 \mathrm{p}_{3 / 2} \\
\text { PEDOT }\end{array}$ & 163.92 & 49.7 & 23.27 & 76.74 & 0 \\
\hline & $\begin{array}{ll}\mathrm{S} & 2 \mathrm{p}_{1 / 2} \\
\text { PEDOT }\end{array}$ & 165.14 & 27.04 & & & \\
\hline & S 2p $\mathrm{p}_{3 / 2}$ PSS- & 167.81 & 10.27 & & & \\
\hline & $\mathrm{S} 2 \mathrm{p}_{1 / 2}$ PSS- & 169.01 & 5.59 & & & \\
\hline & $\mathrm{S} 2 \mathrm{p}_{3 / 2} \mathrm{PSSH}$ & 168.7 & 4.94 & & & \\
\hline & $\mathrm{S} 2 \mathrm{p}_{1 / 2} \mathrm{PSSH}$ & 169.9 & 2.47 & & & \\
\hline $\begin{array}{l}\text { C4 SWNT-PEDOT:PSS- } \\
\text { EMIM:TFSI-EG }\end{array}$ & $-\mathrm{S}_{\mathrm{PEDOT}}^{2 \mathrm{p}_{3 / 2}}$ & 163.92 & 52.27 & 19.29 & 80.71 & 0 \\
\hline & $\begin{array}{ll}\mathrm{S} & 2 \mathrm{p}_{1 / 2} \\
\text { PEDOT }\end{array}$ & 165.14 & 28.44 & & & \\
\hline & $\mathrm{S} 2 \mathrm{p}_{3 / 2}$ PPS- & 167.8 & 6.91 & & & \\
\hline & $\mathrm{S} 2 \mathrm{p}_{1 / 2}$ PSS- & 169 & 3.76 & & & \\
\hline & $\mathrm{S} 2 \mathrm{p}_{3 / 2} \mathrm{PSSH}$ & 168.56 & 5.75 & & & \\
\hline & $\mathrm{S} 2 \mathrm{p}_{1 / 2} \mathrm{PSSH}$ & 169.76 & 2.87 & & & \\
\hline S1 pristine PEDOT:PSS-GuI & $\mathrm{S} 2 \mathrm{p}_{3 / 2} \mathrm{PSSH}$ & 168.5 & 10.9 & 31.83 & 64.68 & 3.49 \\
\hline & $\mathrm{S} 2 \mathrm{p}_{1 / 2} \mathrm{PSSH}$ & 169.66 & 6.54 & & & \\
\hline & $\begin{array}{ll}\mathrm{S} & 2 \mathrm{p}_{3 / 2} \\
\text { PEDOT }\end{array}$ & 163.82 & 41.89 & & & \\
\hline & $\begin{array}{l}\mathrm{S} \\
\text { PEDOT }\end{array}$ & 165.05 & 22.79 & & & \\
\hline & $\mathrm{S} 2 \mathrm{P}_{3 / 2} \mathrm{SH} 2$ & 162.59 & 2.26 & & & \\
\hline & $\mathrm{S} 2 \mathrm{p}_{1 / 2} \mathrm{SH} 2$ & 163.79 & 1.23 & & & \\
\hline & $\mathrm{S} 2 \mathrm{p}_{3 / 2}$ PSS- & 168.1 & 9.32 & & & \\
\hline & $\mathrm{S} 2 \mathrm{p}_{1 / 2}$ PSS- & 169.3 & 5.07 & & & \\
\hline S2 SWNT-PEDOT:PSS-GuI & $\mathrm{S} 2 \mathrm{p}_{3 / 2} \mathrm{PSSH}$ & 168.35 & 9.62 & 26.4 & 72.79 & 0.8 \\
\hline
\end{tabular}

$206 \mid \mathrm{P}$ a g e 


\begin{tabular}{|c|c|c|c|c|c|c|}
\hline & $\mathrm{S} 2 \mathrm{p}_{1 / 2} \mathrm{PSSH}$ & 169.51 & 5.77 & & & \\
\hline & $\begin{array}{l}\mathrm{S} \\
\text { PEDOT }\end{array}$ & 163.9 & 47.14 & & & \\
\hline & $\begin{array}{l}\text { S } 2 \mathrm{p}_{1 / 2} \\
\text { PEDOT }\end{array}$ & 165.13 & 25.65 & & & \\
\hline & $\mathrm{S} 2 \mathrm{P}_{3 / 2} \mathrm{SH} 2$ & 162.86 & 0.52 & & & \\
\hline & $\mathrm{S} 2 \mathrm{p}_{1 / 2} \mathrm{SH} 2$ & 164.06 & 0.28 & & & \\
\hline & S 2p p $3 / 2$ PSS- & 167.95 & 7.13 & & & \\
\hline & $\mathrm{S} 2 \mathrm{p}_{1 / 2}$ PSS- & 169.15 & 3.88 & & & \\
\hline S3 PEDOT:PSS-EMIM:TFSI-GuI & $\mathrm{S} 2 \mathrm{p}_{3 / 2} \mathrm{PSSH}$ & 168.59 & 9.34 & 33.86 & 61.49 & 4.66 \\
\hline & $\mathrm{S} 2 \mathrm{p}_{1 / 2} \mathrm{PSSH}$ & 169.75 & 5.6 & & & \\
\hline & $\begin{array}{l}\mathrm{S}^{2 \mathrm{p}_{3 / 2}} \\
\text { PEDOT }\end{array}$ & 163.89 & 39.82 & & & \\
\hline & $\begin{array}{l}\mathrm{S} 2 \mathrm{p}_{1 / 2} \\
\text { PEDOT }\end{array}$ & 165.09 & 21.67 & & & \\
\hline & $\mathrm{S} 2 \mathrm{p}_{3 / 2} \mathrm{SH} 2$ & 162.59 & 3.02 & & & \\
\hline & $\mathrm{S} 2 \mathrm{p}_{1 / 2} \mathrm{SH} 2$ & 163.79 & 1.64 & & & \\
\hline & S 2p p $3 / 2$ PSS- & 168.19 & 12.25 & & & \\
\hline & $\mathrm{S} 2 \mathrm{P}_{1 / 2}$ PSS- & 169.39 & 6.67 & & & \\
\hline $\begin{array}{l}\text { S4 } \\
\text { EMIM:TFSI-GUI }\end{array}$ & $\mathrm{S} 2 \mathrm{p}_{3 / 2} \mathrm{PSSH}$ & 168.54 & 10.29 & 23.82 & 66.61 & 9.57 \\
\hline & $\mathrm{S} 2 \mathrm{p}_{1 / 2} \mathrm{PSSH}$ & 169.7 & 6.17 & & & \\
\hline & $\begin{array}{l}\mathrm{S} \\
\text { PEDOT }\end{array}$ & 163.72 & 43.14 & & & \\
\hline & $\begin{array}{l}\mathrm{S} \\
\text { PEDOT }\end{array}$ & 164.95 & 23.47 & & & \\
\hline & $\mathrm{S} 2 \mathrm{p}_{3 / 2} \mathrm{SH} 2$ & 162.66 & 6.2 & & & \\
\hline & $\mathrm{S} 2 \mathrm{p}_{1 / 2} \mathrm{SH} 2$ & 163.86 & 3.37 & & & \\
\hline & S 2p p $3 / 2$ PSS- & 168.14 & 4.77 & & & \\
\hline
\end{tabular}

207| P a g e 


\begin{tabular}{|l|l|l|l|l|l|l|}
\hline & S 2 2 $p_{1 / 2}$ PSS & 169.34 & 2.59 & & & \\
\hline
\end{tabular}

Regarding PEDOT:PSS films some of the evidence used to detect the desulphonation is carried out via XPS by analysing a decreased $\mathrm{O}$ 1s peak intensity in aging PSS due to thermal or optically induced degradation. ${ }^{196}$ Although Oxygen 1s analysis is useful it doesn't give a complete narrative of the sulphur removal. This is because the products of desulphonation are $\mathrm{SO}_{3}$ and $\mathrm{H}_{2} \mathrm{SO}_{4} / \mathrm{HSO}_{4}$ with $\mathrm{BE}$ around $167-$ $169 \mathrm{eV}^{196,581}$ it's difficult to determine desulphonation through analysis of the change in the S $2 p$ spectrum to identify this because the products have BEs in the same region as PSS $167-171 \mathrm{eV}{ }^{582583}$ In contrast the $\mathrm{SH}_{2}$ is in $2^{-}$oxidation state observed in this study is in a much lower BE $(162.5 \mathrm{eV})$ than PEDOT or PSS thereby giving a more direct evidence. ${ }^{306}$ Observing the reduced product of desulphurization gives more tangible evidence and it would be interesting to see if the desulphurization process can studied further in PEDOT:PSS thermoelectric research to induce a more significant selective PSS removal. By removing the sulfonate and sulfonic acid groups from PSS, the coulombic interaction between PSS and PEDOT $^{+}$is reduced therefore only a suitable solvent to selectively wash off PSS from the film or solution would be required. ${ }^{112,323}$ Figure 7.6.3.2 depicts the proposed mechanism for desulphurization catalysed by the guanidinium and Equation 6 below the subsequent reduction of the sulphone to sulphide by Guanidine.

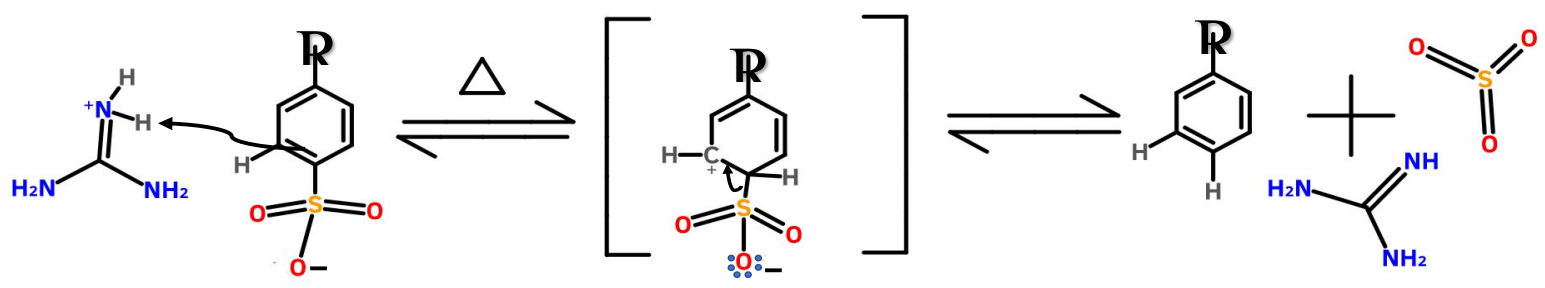

Figure 7.6.3.2 depicting a desulphonation route using guanidinium ion as the catalyst. The $\Delta$ symbol depicts heat at $160{ }^{\circ} \mathrm{C}$ in ethylene glycol. $R$ denotes the rest of the polymer. 


$$
\mathrm{SO}_{3}+8 \mathrm{H} \rightarrow \mathrm{H}_{2} \mathrm{~S}+3 \mathrm{H}_{2} \mathrm{O}
$$

As was evidenced in Figure 7.6.2.2 the N 1s peak had a BE at $398 \mathrm{eV}$ labelled "sign of deprotonation". This gives further evidence the dissociated guanidinium ions underwent redox reactions. ${ }^{486}$ In the desulphonation mechanism (Figure 7.6.3.2) the electron rich $\pi$ electrons of benzenesulfonic acid attacks the electron deficient $\mathrm{H}$ from the $\mathrm{N}^{+}$in a nucleophilic elimination reaction whereby $\mathrm{SO}_{3}$ is the elimination product and $\mathrm{H}^{+}$added onto the benzene ring to replace it. ${ }^{584,585}$ The now basic guanidine and guanidinium (source of $\mathrm{H}^{+}$) is involved in a further reaction whereby it guanidine reduces the $\mathrm{SO}_{3}$ group into $\mathrm{SH}_{2}$ (refer to Equation 6). The $\mathrm{SH}_{2}$ then is detected by the $\mathrm{S} 2 \mathrm{p}$ in films it remained adsorbed on the film surface.

\subsection{Conclusions}

A SWNT-PEDOT:PSS-EMIM:TFSI composite was synthesized with significantly improved performance relative to the pristine PEDOT:PSS film. The film with the highest $\mathrm{PF}$ of $236 \mu \mathrm{W} \mathrm{m} \mathrm{m}^{-1} \mathrm{~K}^{-2}$ at $140{ }^{\circ} \mathrm{C}$, was the SWNT-PEDOT:PSSEMIM:TFSI-GuI. The molecular improvements that led to the extremely high electrical conductivity of $3665 \mathrm{~S} \mathrm{~cm}^{-1}$ and almost doubled Seebeck coefficient of 22 $\mu \mathrm{V} \mathrm{K}^{-1}$ was elucidated. XPS studies showed that films treated post treated with ethylene glycol or the guanidinium iodide solution (GuI) caused significant phase separation between PEDOT and PSS and thereby resulted in selective removal of PSS. XPS analysis on S 2p peaks gave evidence of desulphonation of PSS by the presence of the $\mathrm{SH}_{2}$ peak at $\mathrm{BE}(162.5 \mathrm{eV})$. It is thought that the desulphonation of PSS may have reduced the coulombic attraction between $\mathrm{PSS}^{-}$and $\mathrm{PEDOT}^{+}$even further than the ethylene glycol treatment due to removal the anionic component in PSS- 
The UV-Vis-NIR studies and XPS spectra revealed some of the explanation of the improved Seebeck coefficient was due to a redox reaction between $\mathrm{I}^{-}$and thiophene backbone which led to a reduction of charge carriers in which it is known to improve the Seebeck coefficient in PEDOT:PSS. Still, the simultaneous improvement in both Seebeck coefficient and electrical conductivity in GuI treated films is thought to be because of guanidinium ions that have adsorbed on the film surface as evidenced by $\mathrm{N}$ 1s peaks in which they may have cation- $\pi$ interactions whereby its presence increases bulk carrier concentration even though some of the thiophene carriers (bipolaron) were reduced to some (polarons).

Further research into seeing the effects of increasing or reducing the concentration of GuI solution used to treat the films, and or the temperature the films were treated at would potentially allow for the PF to be further optimised. Other studies that would be important is finding out how improving the bulk carrier concentration while reducing the thiophene carrier concentration would affect the optimised PF and further using different variations of guanidinium halides and or other derivatives would potentially allow for new strategies at improving the PF. 


\section{Chapter 8 Outlook and conclusion}

Thermal conductivity measurements were not conducted in this research project. This is a limitation because a full thermoelectric characterization was not obtained. The decision to focus on using the power factor to determine the extent of improvements to the thermoelectric properties of the films was due to difficulties measuring the thermal conductivity on thin films less than $70 \mathrm{~nm}$ thick. ${ }^{586}$ It is possible to estimate ZT by using the thermal conductivity values from literature for similar materials however this will invariably lead to inaccuracies. This is because the treatment conditions used in the experimental chapters in this project are unique enough that the materials properties would deviate from reported literature values. The extent of deviation between real and estimated thermal conductivity may be great especially when considering the most electrically conductive film (S4 SWNT-EMIM:TFSIPEDOT:PSS) which had an electrical conductivity of $3665 \mathrm{~S} \mathrm{~cm}^{-1}$. Even if the lattice contribution to the thermal conductivity does not increase substantially the electronic contribution will invariably increase thereby making it difficult to accurately estimate it from reported literature values pertaining to similar materials. ${ }^{587}$ Therefore, future studies exploring and developing methods to measure thermal conductivity of thin films reliably and accurately is required. ${ }^{588}$ In terms of the measurements of the Seebeck coefficient, future studies will be conducted with temperature difference of 5 ${ }^{\circ} \mathrm{C}$ between each of the three measurement temperatures instead for three temperatures instead of $10^{\circ} \mathrm{C}$ as indicated in chapter 3.3.5.

It is important to explore why the Seebeck coefficient within any study in this project remained less than $50 \mu \mathrm{V} \mathrm{K}^{-1}$ at temperatures below $150{ }^{\circ} \mathrm{C}$. The significance of 150 ${ }^{\circ} \mathrm{C}$ is due to thermal cycling stability considerations for maintained performance for low temperature potential application such as integration into solar or geothermal systems. ${ }^{589}$ In literature, values higher than $100 \mu \mathrm{V} \mathrm{K}^{-1}$ for $\mathrm{NaBH}_{4}$, tetrakis dimethylamino ethylene, and hydrazine treated PEDOT:PSS films can be observed. ${ }^{468}$ There is however a trade-off that usually follows whereby the electrical conductivity reduces to significantly lower values leading to low power factors. In a study by 
Massonnet et al, a film treated with $\mathrm{NaBH}_{4}$ improved the Seebeck coefficient from 18 $\mu \mathrm{V} \mathrm{K}^{-1}$ to $104 \mu \mathrm{V} \mathrm{K}^{-1}$. ${ }^{468}$ The electrical conductivity however reduced to $0.2 \mathrm{~S} \mathrm{~cm}^{-1}$. A similar loss in conductivity was observed for the films treated with hydrazine and Tetrakis dimethylamino ethylene and hydrazine. ${ }^{468}$ The concentration for the $\mathrm{NaBH}_{4}$ was high at $10 \% \mathrm{wt}$ in aqueous solution. This is a high concentration and since then studies exploring optimisation studies by finding ideal concentrations have been published to reach a trade-off that improves the power factor. ${ }^{128,363,590}$ In the study outlined in chapter 5 , a $1 \% \mathrm{NaBH}_{4}$ solution was used to find the effects of the reducing agent on a PEDOT:PSS-ionic liquid composite instead of for optimising the trade-off between Seebeck coefficient and electrical conductivity. Further studies to discover the optimal concentration of $\mathrm{NaBH}_{4}$ treatment on PEDOT:PSS-ionic liquid composites would be desirable. During the preliminary experiments, results showed that higher concentrations of $\mathrm{NaBH}_{4}$ solution led to higher Seebeck coefficients above $70 \mu \mathrm{V} \mathrm{K}^{-1}$ however the films had significantly reduced electrical conductivities and in some cases, it was not possible to reliably measure the films on the ULVAC-ZEM-3 due to the high resistance. The ULVAC-ZEM-3 has a measurement limit of $100 \mathrm{k} \Omega$.

In this study there are four chapters whereby different treatments and conditions were explored yielding different improvements to the thermoelectric properties to PEDOT:PSS and its composites. There are key successes as outline in chapter 6 and 7. The results outlined in chapter 6 provided a fast and novel route to an $n$ type SWNT/PEDOT:PSS composite. Heat and a reducing agent (tetra butyl ammonium hydroxide) were used to aid reduction of the films. The presence of SWNTs in the films facilitated as the electron acceptor into the composite. It would be interesting to observe how the temperature the films are treated at would affect the $\mathrm{n}$ type characteristics as well as determine the minimum temperature that allows for $n$ type characteristics to evolve while also varying the treatment time to see how that would also affect $\mathrm{n}$ type characteristics. This would allow for optimization of the treatment process as well as give insight on the extent of treatment time/temperature affects the size and charge of the Seebeck coefficient. It would of course be interesting to try to repeat the experiment utilizing different types of carbon nanotubes and even try the Tetrabutyle ammonium hydroxide treatment on separated/purified carbon nanotubes such as semiconducting carbon nanotubes as their composites with PEDOT:PSS have 
sometimes resulted in higher Seebeck coefficients than the mixed or metallic carbon nanotubes. ${ }^{591}$ For these films to gain an $\mathrm{n}$ type characteristic while also being high would be interesting. Another important future study would be to find a way to improve the electrical conductivity of the n type films.

In chapter 7 several novel PEDOT:PSS composites were developed and further treated with a guanidinium iodide solution at a set temperature. The success of the study showed potential routes to simultaneously improve the Seebeck coefficient and electrical conductivity of PEDOT:PSS and its composites. Future studies looking into derivatives of guanidinium iodide such as guanidinium chloride would be important to understand the relationship the anion has on the effects on the thermoelectric properties of the films in chapter 7. Further studies looking at how different treatment temperatures and concentrations of guanidinium iodide will be useful in understanding the kinetics of the reduction. For example, a question that has not been fully explored is whether the simultaneous improvement to the Seebeck coefficient and electrical conductivity is primarily due to the weak reducing activity of guanidinium iodide or because of improved carrier properties of the film. If a study shows that certain derivatives of guanidinium iodide induce a simultaneous improvement on the electrical conductivity and Seebeck coefficient then more generalized statements can be made pertaining to the proposed materials class. It's therefore also important to measure carrier concentration and carrier mobility and employ the same experiment at elevated concentrations and temperature to study the effects. It is useful to conduct a study looking into the stability of these films across a timeline of several months to understand the relevance of the materials in real world application. This would involve measuring how and if the thermoelectric properties degrade across time as well as effects of the environmental conditions may affect the physical properties of the films such as mechanical deformations.

To address the viability of the materials developed in this research endeavor, fabricating functional thermoelectric devices is required. It is possible to use the $\mathrm{n}$ type materials developed in chapter 6 with the high performing $p$ type materials developed in chapter 7 to fabricate thermoelectric devices and analyze their performance. Key 
issues to be explored pertain to the size and geometry of the materials. The thin films deposited on non-conductivity glass substrates have their thermoelectric properties measured as $2.2 \mathrm{~cm}$ length by $1.1 \mathrm{~cm}$ width. The in plane thermoelectric properties are determined for several reasons. The first is due to difficulty in measuring the out plane electrical conductivity of the films due to the thickness being extremely small below $100 \mathrm{~nm}$. The second is because thermometric devices require a temperature difference to be maintained to power electricity generation. It is clear to see that films with thicknesses below $100 \mathrm{~nm}$ will make it difficult for a temperature difference to be maintained. Therefore, the in plane thermoelectric properties are usually considered for organic and hybrid thermoelectric devices. ${ }^{592}$ For the materials in this study some experiments that may allow for testing thermoelectric devices could be realized as follows. A nonconductive glass or polyethylene terephthalate (PET) material can be used as the base of the thermoelectric device. The $2.2 \mathrm{by} 1.1 \mathrm{~cm}$ testing pieces as shown in Figure 3.5.5.1 can be fixed at the base of a glass or PET material. The testing pieces can be aligned as alternative $\mathrm{p}$ and $\mathrm{n}$ types and connected electrically in series and thermally in parallel. Experiments showing different numbers of these pieces (legs) can then be conducted to see how many are required to generate a particular power output. Moreover, methods looking at ways to eject heat with optimised device designs would help make device architecture more relevant to organic and hybrid thermoelectric systems. 


\section{References}

1. Cowen, L.M., Atoyo, J., Carnie, M.J., Baran, D., and Schroeder, B.C. (2017). Review-Organic Materials for Thermoelectric Energy Generation. ECS J. Solid State Sci. Technol. 6, N3080-N3088.

2. Atoyo, J., Burton, M.R., McGettrick, J., and Carnie, M.J. (2020). Enhanced Electrical Conductivity and Seebeck Coefficient in PEDOT:PSS via a Two-Step Ionic liquid and NaBH4 Treatment for Organic Thermoelectrics. Polym. 2020, Vol. 12, Page 559 12, 559.

3. Green, F. (2018). Anti-fossil fuel norms. 103-116.

4. Erickson, P., Lazarus, M., and Piggot, G. (1990). in climate policy. Nat. Clim. Chang.

5. Iniyan, S., Sargunam, J., and Samuel, A.A. Energy Models for Renewable Energy Utilization and To Replace fossil fuels Energy Models for Renewable Energy Utilization and To Replace fossil fuels Iniyan S Department of Mechanical Engineering, Bharath Institute of Science Key words : Energy Models , .

6. Musa, S.D., Zhonghua, T., Ibrahim, A.O., and Habib, M. (2017). China 's energy status : A critical look at fossils and renewable options. Renew. Sustain. Energy Rev., 0-1.

7. Kåberger, T. (2018). Progress of renewable electricity replacing fossil fuels. Glob. Energy Interconnect. 1, 48-52.

8. Perera, F. (2018). Pollution from Fossil-Fuel Combustion is the Leading Environmental Threat to Global Pediatric Health and Equity : Solutions Exist.

9. Elankovan, R., Suresh, S., and Karthick, K. (2019). Modelling and O $215 \mid \mathrm{P}$ a g e 
ptimization of $\mathrm{H}$ eat $\mathrm{T}$ ransfer $\mathrm{C}$ oefficients for $\mathrm{H}$ ot and $\mathrm{C}$ old $\mathrm{S}$ ides of Thermoelectric Generator ( TEG ) system. 1174-1184.

10. Sarbu, I. (2017). A comprehensive review of solar thermoelectric cooling systems. 1-21.

11. Yang, L., Chen, Z., Dargusch, M.S., and Zou, J. (2017). High Performance Thermoelectric Materials : Progress and Their Applications. 1701797, 1-28.

12. Yang, J., and Caillat, T. (2006). T hermoelectric Materials for Space. 31, 224 229.

13. Esposito, M. (2010). Efficiency at Maximum Power of Low-Dissipation Carnot Engines. 150603, 1-4.

14. Woods, M.P., Huei, N., Ng, Y., and Wehner, S. (2019). The maximum efficiency of nano heat engines depends on more than temperature.

15. Pietzonka, P., and Seifert, U. (2018). Universal Trade-Off between Power, Efficiency, and Constancy in Steady-State Heat Engines. Phys. Rev. Lett. 120, 190602.

16. Bjørk, R., and Nielsen, K.K. (2018). The maximum theoretical performance of unconcentrated solar photovoltaic and thermoelectric generator systems. Energy Convers. Manag. 156, 264-268.

17. Zhang, Y., Heo, Y., Park, M., and Park, S. (2019). Recent Advances in Organic Thermoelectric Materials : Principle Mechanisms and Emerging Carbon-Based Green Energy Materials.

18. Vitoratos, E., Sakkopoulos, S., Dalas, E., Paliatsas, N., Karageorgopoulos, D., Petraki, F., Kennou, S., and Choulis, S.A. (2009). Thermal degradation mechanisms of PEDOT:PSS. Org. Electron. physics, Mater. Appl. 10, 61-66.

19. Lappas, V., Tsourdos, A., Kindylides, S., and Kostopoulos, V. (2019). A Low Cost Thermoelectric Generator for Small Satellites. 1-14.

20. Zhu, W., Tu, Y., and Deng, Y. (2018). Multi-parameter optimization design of 
thermoelectric harvester based on phase change material for space generation. Appl. Energy 228, 873-880.

21. Li, L., Gao, X., Zhang, G., Xie, W., Wang, F., and Yao, W. (2019). Combined solar concentration and carbon nanotube absorber for high performance solar thermoelectric generators. Energy Convers. Manag. 183, 109-115.

22. Blackburn, J.L., Ferguson, A.J., Cho, C., and Grunlan, J.C. (2018). CarbonNanotube-Based Thermoelectric Materials and Devices. Adv. Mater. 30, 1-35.

23. Wang, H., Ail, U., Gabrielsson, R., Berggren, M., and Crispin, X. (2015). Ionic Seebeck Effect in Conducting Polymers. 1-6.

24. Mettan, X., Pisoni, R., Matus, P., Pisoni, A., Jacimovic, J., Náfrádi, B., Spina, M., Pavuna, D., Forró, L., and Horváth, E. (2015). Tuning of the Thermoelectric Figure of Merit of CH 3 NH 3 MI $3(\mathrm{M}=\mathrm{Pb}, \mathrm{Sn})$ Photovoltaic Perovskites. J. Phys. Chem. C 3, 150503102129004.

25. Sun, P., Wei, B., Zhang, J., Tomczak, J.M., Strydom, A.M., Søndergaard, M., Iversen, B.B., and Steglich, F. (2015). Large Seebeck effect by charge-mobility engineering. Nat. Commun. 6, 7475.

26. Schierning, G., Chavez, R., Schmechel, R., Balke, B., Rogl, G., and Rogl, P. (2015). Concepts for medium-high to high temperature thermoelectric heat-toelectricity conversion : a review of selected materials and basic considerations of module design Concepts for medium-high to high temperature thermoelectric heat-to-electricity conversio.

27. Sun, C., Soup, G., Choi, H., Jun, Y., and Man, H. (2018). Structural design of a fl exible thermoelectric power generator for wearable applications. Appl. Energy 214, 131-138.

28. Pietrzyk, K., Ohara, B., Watson, T., Gee, M., Avalos, D., and Lee, H. (2016). Thermoelectric module design strategy for solid-state refrigeration. Energy 114, 823-832. 
29. Karri, N.K., and Mo, C. (2018). Reliable Thermoelectric Module Design under Opposing Requirements from Structural and Thermoelectric Considerations. J. Electron. Mater. 47, 3127-3135.

30. Mohsenzadeh, M., Shafii, M.B., and Jafari, H. (2017). A novel concentrating photovoltaic/thermal solar system combined with thermoelectric module in an integrated design. Renew. Energy.

31. Skomedal, G., Holmgren, L., Middleton, H., Eremin, I.S., Isachenko, G.N., Jaegle, M., Tarantik, K., Vlachos, N., Manoli, M., Kyratsi, T., et al. (2016). Design, assembly and characterization of silicide-based thermoelectric modules. Energy Convers. Manag. 110, 13-21.

32. Satoh, N., Otsuka, M., Ohki, T., Ohi, A., Sakurai, Y., Yamashita, Y., and Mori, T. (2018). Organic $\pi$-type thermoelectric module supported by photolithographic mold: a working hypothesis of sticky thermoelectric materials. Sci. Technol. Adv. Mater. 19, 517-525.

33. Meng, J.-H., Wang, X.-D., and Chen, W.-H. (2016). Performance investigation and design optimization of a thermoelectric generator applied in automobile exhaust waste heat recovery. Energy Convers. Manag. 120, 71-80.

34. Ibañez-puy, M., Bermejo-busto, J., Martín-gómez, C., Vidaurre-arbizu, M., and Sacristán-fernández, J.A. (2017). Thermoelectric cooling heating unit performance under real conditions. 200, 303-314.

35. Cui, L., Miao, R., Wang, K., Thompson, D., Zotti, L.A., Cuevas, J.C., Meyhofer, E., and Reddy, P. (2018). Peltier cooling in molecular junctions. Nat. Nanotechnol. 13.

36. Jin, W., Liu, L., Yang, T., Shen, H., Zhu, J., Li, Q., Chi, L., Di, C., and Zhu, D. (2018). Exploring Peltier effect in organic thermoelectric fi lms. Nat. Commun., $1-6$.

37. Liu, Z., Zhang, L., Gong, G., Li, H., and Tang, G. (2015). Review of solar thermoelectric cooling technologies for use in zero energy buildings. Energy Build. 102, 207-216. 
38. Liu, D., Zhao, F., Yang, H., and Tang, G. (2015). Thermoelectric mini cooler coupled with micro thermosiphon for CPU cooling system. Energy, 1-8.

39. Snyder, G.J., Khanna, R., Toberer, E.S., Heinz, N.A., and Seifert, W. (2016). Improved thermoelectric cooling based on the Thomson effect. In Proc.SPIE.

40. Tsai, H., and Le, P.T. (2016). Self-sufficient energy recycling of light emitter diode / thermoelectric generator module for its active-cooling application. ENERGY Convers. Manag. 118, 170-178.

41. Im, D.H., Chan, H., Kwang, K., Kim, J., and Hee, K. (2019). Study on the Design of a New Heatsink Cooling System for Thermoelectric Dehumidifier. Int. J. Precis. Eng. Manuf.

42. He, W., Zhang, G., Zhang, X., Ji, J., Li, G., and Zhao, X. (2015). Recent development and application of thermoelectric generator and cooler. Appl. Energy 143, 1-25.

43. Zoui, M.A., Bentouba, S., Stocholm, J.G., and Bourouis, M. (2020). A Review on Thermoelectric Generators: Progress and Applications. Energies 13.

44. Snyder, G.J., and Toberer, E.S. (2008). Complex thermoelectric materials. 7, $105-114$.

45. Chen, Z., Shi, X., Zhao, L., and Zou, J. (2018). High-performance SnSe thermoelectric materials : progress and future challenge. Prog. Mater. Sci.

46. Meng, F., Chen, L., Feng, Y., and Xiong, B. (2017). Thermoelectric generator for industrial gas phase waste heat recovery. Energy 135, 83-90.

47. He, W., Wang, S., Zhao, Y., and Li, Y. (2016). Effects of heat transfer characteristics between fluid channels and thermoelectric modules on optimal thermoelectric performance. ENERGY Convers. Manag. 113, 201-208.

48. Ramousse, J., Sgorlon, D., Fraisse, G., and Perier-Muzet, M. (2015). Analytical optimal design of thermoelectric heat pumps. Appl. Therm. Eng. 82, 48-56. 
49. Liu, D., Cai, Y., and Zhao, F.-Y. (2017). Optimal design of thermoelectric cooling system integrated heat pipes for electric devices. Energy 128, 403-413.

50. Dongxu, J., Zhongbao, W., Pou, J., Mazzoni, S., Rajoo, S., and Romagnoli, A. (2019). Geometry optimization of thermoelectric modules: Simulation and experimental study. Energy Convers. Manag. 195, 236-243.

51. Sootsman, J.R., Chung, D.Y., and Kanatzidis, M.G. (2009). New and Old Concepts in Thermoelectric Materials Angewandte. 8616-8639.

52. Min, G.A.O. (2010). ZT Measurements Under Large Temperature Differences. $39,1782-1785$.

53. Snyder, G.J., and Snyder, A.H. (2017). Figure of merit ZT of a thermoelectric device defined from materials properties. Energy Environ. Sci. 10, 2280-2283.

54. Zuo, G., Liu, X., Fahlman, M., and Kemerink, M. (2017). High Seebeck Coefficient in Mixtures of Conjugated Polymers. 1703280, 1-7.

55. Petsagkourakis, I., Pavlopoulou, E., Cloutet, E., Chen, Y.F., Liu, X., Fahlman, M., Berggren, M., Crispin, X., Dilhaire, S., Fleury, G., et al. (2018). Correlating the Seebeck coefficient of thermoelectric polymer thin films to their charge transport mechanism. Org. Electron. 52, 335-341.

56. Zuo, G., Li, Z., Wang, E., and Kemerink, M. (2017). High Seebeck Coefficient and Power Factor in n-Type Organic Thermoelectrics. 1700501, 1-6.

57. Schmidt, R., Wilken, F., Nunner, T.S., and Brouwer, P.W. (2018). Boltzmann approach to the longitudinal spin Seebeck effect. Phys. Rev. B 98, 134421.

58. Zou, Y., Huang, D., Meng, Q., Di, C., and Zhu, D. (2018). Correlation between Seebeck coefficient and transport energy level in poly(3-hexylthiophene). Org. Electron. 56, 125-128.

59. Sharma, V.K., Gupta, D.C., and Half-heuslers, Z. (2019). Applicability of semiclassical Boltzmann transport theory in understanding the thermoelectric properties of $\mathrm{ZrNiSn}$ and $\mathrm{ZrNiPb}$ half-heuslers Applicability of Semi-classical Boltzmann Transport Theory in Understanding the Thermoelectric Properties 
of ZrNiS. 030420.

60. Fiorentini, M., and Bonini, N. (2016). Thermoelectric coefficients of \$n \$-doped silicon from first principles via the solution of the Boltzmann transport equation. Phys. Rev. B 94, 85204.

61. Zhang, Q., Chere, E.K., Mcenaney, K., Yao, M., Cao, F., Ni, Y., Chen, S., Opeil, C., Chen, G., and Ren, Z. (2015). Enhancement of Thermoelectric Performance of n-Type $\mathrm{PbSe}$ by $\mathrm{Cr}$ Doping with Optimized Carrier Concentration. $1-8$.

62. Dun, C., Hewitt, C.A., Huang, H., Xu, J., Zhou, C., Huang, W., Cui, Y., Zhou, W., Jiang, Q., and Carroll, D.L. (2015). Flexible n-type thermoelectric films based on $\mathrm{Cu}$-doped $\mathrm{Bi} 2 \mathrm{Se} 3$ nanoplate and Polyvinylidene Fluoride composite with decoupled Seebeck coefficient and electrical conductivity. Nano Energy $18,306-314$.

63. Kim, H., Gibbs, Z.M., Tang, Y., Wang, H., Snyder, G.J., Kim, H., Gibbs, Z.M., Tang, Y., Wang, H., and Snyder, G.J. (2015). Characterization of Lorenz number with Seebeck coefficient measurement Characterization of Lorenz number with Seebeck coefficient measurement. 041506, 1-6.

64. Wang, X., Askarpour, V., Maassen, J., Lundstrom, M., Wang, X., Askarpour, V., Maassen, J., and Lundstrom, M. (2018). On the calculation of Lorenz numbers for complex thermoelectric materials On the calculation of Lorenz numbers for complex thermoelectric materials. 055104.

65. Secco, R.A. (2017). Thermal conductivity and Seebeck coefficient of Fe and Fe-Si alloys: Implications for variable Lorenz number. Phys. Earth Planet. Inter. 265, 23-34.

66. Kim, H.S., Wang, T., Liu, W., and Ren, Z. (2016). Engineering Thermal Conductivity for Balancing Between Reliability and Performance of Bulk Thermoelectric Generators. 3678-3686. 
67. Smith, P.M., Su, L., Gong, W., Nakamura, N., Reeja-Jayan, B., and Shen, S. (2018). Thermal conductivity of poly(3,4-ethylenedioxythiophene) films engineered by oxidative chemical vapor deposition (oCVD). RSC Adv. 8, 19348-19352.

68. Hanus, R., Agne, M.T., Rettie, A.J.E., Chen, Z., Tan, G., Chung, D.Y., Kanatzidis, M.G., Pei, Y., Voorhees, P.W., and Snyder, G.J. (2019). Lattice Softening Significantly Reduces Thermal Conductivity and Leads to High Thermoelectric Efficiency. 1900108, 1-10.

69. Cho, C., Stevens, B., Hsu, J., Bureau, R., Hagen, D.A., Regev, O., Yu, C., and Grunlan, J.C. (2015). Completely Organic Multilayer Thin Film with Thermoelectric Power Factor Rivaling Inorganic Tellurides. 1-6.

70. Wolf, M., Hinterding, R., and Feldhoff, A. (2019). High power factor vs. high zT-A review of thermoelectric materials for high-temperature application. Entropy 21.

71. Liu, S., Li, H., and He, C. (2019). Simultaneous enhancement of electrical conductivity and seebeck coef fi cient in organic thermoelectric SWNT / PEDOT : PSS nanocomposites. Carbon N. Y. 149, 25-32.

72. Liu, W., Kim, H.S., Jie, Q., and Ren, Z. (2016). Importance of high power factor in thermoelectric materials for power generation application: A perspective. Scr. Mater. 111, 3-9.

73. Xiao, C., Li, Z., Li, K., Huang, P., and Xie, Y. (2014). Decoupling Interrelated Parameters for Designing High Performance Thermoelectric Materials.

74. Sciences, M., and Iowa, A. (2016). 06-03-2016 Charge carrier effective mass and concentration derived from combination of Seebeck coefficient and $125 \mathrm{Te}$ NMR measurements in complex tellurides E. M. Levin. 50, 1-15.

75. Madavali, B., Shin, D., Kim, D., and Hong, S. (2019). Intermetallics Enhanced thermoelectric properties by e ff ective decoupling of electrical, thermal properties and seebeck coe ffi cient through the addition of rare earth sesquioxides in p-type BiSbTe alloys. Intermetallics 105, 139-145. 
76. Fiorentini, M., and Bonini, N. (2016). Thermoelectric coefficients of n -doped silicon from first principles via the. 085204, 1-11.

77. Zhou, Y., Gong, X., Xu, B., and Hu, M. (2017). Decouple electronic and phononic transport in nanotwinned structures : a new strategy for enhancing the fi gure-of-merit of thermoelectrics $\uparrow .9-17$.

78. Li, H., Plunkett, E., Cai, Z., Qiu, B., Wei, T., Chen, H., Thon, S.M., Reich, D.H., Chen, L., and Katz, H.E. (2019). Dopant-Dependent Increase in Seebeck Coefficient and Electrical Conductivity in Blended Polymers with Offset Carrier Energies. 1800618, 1-11.

79. Kim, C., Young, J., Hwan, D., Tae, J., Humberto, D., Kim, T., and Kim, H. (2017). Journal of Industrial and Engineering Chemistry Decoupling of thermal and electrical conductivities by adjusting the anisotropic nature in tungsten diselenide causing signi fi cant enhancement in thermoelectric performance. J. Ind. Eng. Chem.

80. Takabatake, T., and Suekuni, K. (2014). Phonon-glass electron-crystal thermoelectric clathrates : Experiments and theory. 86, 669-716.

81. Nolas, G.S., and Tritt, T.M. (1999). SKUTTERUDITES : A Phonon-GlassElectron Crystal Approach to Advanced Thermoelectric Energy Conversion Applications. 89-116.

82. He, Y., and Galli, G. (2014). Nanostructured Clathrate Phonon Glasses: Beyond the Rattling.

83. Bhattacharya, S., Basu, R., Bhatt, R., Pitale, S., Singh, A., Aswal, D.K., Gupta, S.K., Navaneethan, M., and Hayakawa, Y. (2013). CuCrSe2: a high performance phonon glass and electron crystal thermoelectric material. J. Mater. Chem. A 1, 11289-11294.

84. Nolas, G.S., Slack, G.A., and Schujman, S.B. (2001). Semiconductor Clathrates: A Phonon Glass Electron Crystal Material with Potential for 
Thermoelectric Applications.

85. Snyder, G.J., Christensen, M., Nishibori, E., Caillat, T., and Iversen, B.B. (2004). Disordered zinc in Zn4Sb3 with phonon-glass and electron-crystal thermoelectric properties. Nat. Mater. 3, 458-463.

86. Peng, K., Zhang, B., Wu, H., Cao, X., Li, A., Yang, D., Lu, X., Wang, G., Han, X., Uher, C., et al. (2017). Ultra-high average fi gure of merit in synergistic band engineered $\mathrm{Sn} x \mathrm{Na} 1$ À x Se $0.9 \mathrm{~S} 0.1$ single crystals. $x x x, 1-7$.

87. Koumoto, K., Wang, Y., Zhang, R., Kosuga, A., and Funahashi, R. (2010). Oxide Thermoelectric Materials : A Nanostructuring Approach.

88. Biswas, K. (2017). Low Thermal Conductivity and High Thermoelectric Performance in Sb and Bi Codoped GeTe: Complementary E ff ect of Band Convergence and Nanostructuring.

89. Perumal, S., Roychowdhury, S., and Biswas, K. (2016). Reduction of thermal conductivity through nanostructuring enhances the thermoelectric figure of merit in Ge1-xBixTe. Inorg. Chem. Front. 3, 125-132.

90. Zhang, K., Zhang, Q., Wang, L., Jiang, W., and Chen, L. (2017). Enhanced thermoelectric performance of Se-doped $\mathrm{PbTe}$ bulk materials via nanostructuring and multi-scale hierarchical architecture. J. Alloys Compd. $725,563-572$.

91. Pei, Y., Tan, G., Feng, D., Zheng, L., Tan, Q., Xie, X., Gong, S., Chen, Y., Li, J., He, J., et al. (2016). Integrating Band Structure Engineering with All-Scale Hierarchical Structuring for High Thermoelectric Performance in PbTe System. 201601450, 1-11.

92. Mamur, H., Bhuiyan, M.R.A., Korkmaz, F., and Nil, M. (2017). A review on bismuth telluride ( $\mathrm{Bi} 2$ Te 3 ) nanostructure for thermoelectric applications. Renew. Sustain. Energy Rev., 0-1.

93. See, K.C., Feser, J.P., Chen, C.E., Majumdar, A., Urban, J.J., and Segalman, R.A. (2010). Water-processable polymer-nanocrystal hybrids for 
thermoelectrics. Nano Lett. 10, 4664-4667.

94. Wen, Y., and $\mathrm{Xu}$, J. (2017). Scientific Importance of Water-Processable PEDOT - PSS and Preparation, Challenge and New Application in Sensors of Its Film Electrode: A Review. 1121-1150.

95. Qi, X.-Y., Yan, D., Jiang, Z., Cao, Y.-K., Yu, Z.-Z., Yavari, F., and Koratkar, N. (2011). Enhanced Electrical Conductivity in Polystyrene Nanocomposites at. ACS Appl. Mater. interfaces 3, 3130-3133.

96. Leger, J.M., Aimonino, P., Loriers, J., Dordor, P., and Coqblin, B. (1980). Transport properties of SmO. Phys. Lett. A 80, 325-327.

97. Stallinga, P. (2011). Electronic transport in organic materials: Comparison of band theory with percolation/(variable range) hopping theory. Adv. Mater. 23, $3356-3362$.

98. Lu, N., Li, L., and Liu, M. (2016). A review of carrier thermoelectric-transport theory in organic semiconductors. Phys. Chem. Chem. Phys. 18, 19503-19525.

99. Ortmann, F., Bechstedt, F., and Hannewald, K. (2010). Charge transport in organic crystals: Interplay of band transport, hopping and electron-phonon scattering. New J. Phys. 12.

100. Wang, L., and Beljonne, D. (2013). Flexible surface hopping approach to model the crossover from hopping to band-like transport in organic crystals. J. Phys. Chem. Lett. 4, 1888-1894.

101. Li, H., Wu, Z., and Lusk, M.T. (2014). Dangling bond defects: The critical roadblock to efficient photoconversion in hybrid quantum dot solar cells. J. Phys. Chem. C 118, 46-53.

102. Martin, D.C., Martin, D.C., and Thomas, E.L. (1991). Grain boundaries in extended-chain polymers: Theory and experiment. Philos. Mag. A Phys. Condens. Matter, Struct. Defects Mech. Prop. 64, 903-922. 
103. Santamaria, A., Muñoz, M.E., Fernández, M., and Landa, M. (2013). Electrically conductive adhesives with a focus on adhesives that contain carbon nanotubes. J. Appl. Polym. Sci. 129, 1643-1652.

104. De Silva, P., and Van Voorhis, T. (2018). QM/MM Study of Static and Dynamic Energetic Disorder in the Emission Layer of an Organic Light-Emitting Diode. J. Phys. Chem. Lett. 9, 1329-1334.

105. Zheng, Z., Tummala, N.R., Wang, T., Coropceanu, V., and Brédas, J.L. (2019). Charge-Transfer States at Organic-Organic Interfaces: Impact of Static and Dynamic Disorders. Adv. Energy Mater. 9, 1-7.

106. Kenkre, V.M., and Dunlap, D.H. (1992). Charge transport in molecular solids: Dynamic and static disorder. Philos. Mag. B Phys. Condens. Matter; Stat. Mech. Electron. Opt. Magn. Prop. 65, 831-841.

107. Vehoff, T., Chung, Y.S., Johnston, K., Troisi, A., Yoon, D.Y., and Andrienko, D. (2010). Charge transport in self-assembled semiconducting organic layers: Role of dynamic and static disorder. J. Phys. Chem. C 114, 10592-10597.

108. Baumeier, B., Kirkpatrick, J., and Andrienko, D. (2010). Density-functional based determination of intermolecular charge transfer properties for large-scale morphologies. Phys. Chem. Chem. Phys. 12, 11103-11113.

109. Choi, S.J., Kim, B.K., Lee, T.H., Kim, Y.H., Li, Z., Pop, E., Kim, J.J., Song, J.H., and Bae, M.H. (2016). Electrical and thermoelectric transport by variable range hopping in thin black phosphorus devices. Nano Lett. 16, 3969-3975.

110. Abdalla, H., Zuo, G., and Kemerink, M. (2017). Range and energetics of charge hopping in organic semiconductors. Phys. Rev. B 96, 1-6.

111. Russ, B., Glaudell, A., Urban, J.J., and Chabinyc, M.L. (2016). Organic thermoelectric materials temperature control.

112. Arkhipov, V.I., Heremans, P., Emelianova, E. V., and Bässler, H. (2005). Effect of doping on the density-of-states distribution and carrier hopping in disordered organic semiconductors. Phys. Rev. B - Condens. Matter Mater. Phys. 71, 1-7. 
113. Mozer, A.J., Sariciftci, N.S., Lutsen, L., Vanderzande, D., Österbacka, R., Westerling, M., and Juška, G. (2005). Charge transport and recombination in bulk heterojunction solar cells studied by the photoinduced charge extraction in linearly increasing voltage technique. Appl. Phys. Lett. 86, 1-3.

114. Burgess, R.E. (1956). The statistics of charge carrier fluctuations in semiconductors. Proc. Phys. Soc. Sect. B 69, 1020-1027.

115. Grüning, M., Marini, A., and Rubio, A. (2006). Density functionals from manybody perturbation theory: The band gap for semiconductors and insulators. J. Chem. Phys. 124.

116. Hutchison, G.R., Ratner, M.A., and Marks, T.J. (2005). Intermolecular charge transfer between heterocyclic oligomers. Effects of heteroatom and molecular packing on hopping transport in organic semiconductors. J. Am. Chem. Soc. $127,16866-16881$.

117. Pfeiffer, M., Leo, K., Zhou, X., Huang, J.S., Hofmann, M., Werner, A., and Blochwitz-Nimoth, J. (2003). Doped organic semiconductors: Physics and application in light emitting diodes. Org. Electron. 4, 89-103.

118. Forrest, S., Burrows, P., and Thompson, M. (2000). The dawn of organic electronics. IEEE Spectr. 37, 29-34.

119. Zhang, H., Dou, C., Pal, L., and Hubbe, M.A. (2019). Review of electrically conductive composites and films containing cellulosic fibers or nanocellulose. BioResources 14, 7494-7542.

120. Wang, Y., Ding, Y., Guo, X., and Yu, G. (2019). Conductive polymers for stretchable supercapacitors. Nano Res. 12, 1978-1987.

121. Jin Bae, E., Hun Kang, Y., Jang, K.-S., and Yun Cho, S. (2016). Enhancement of Thermoelectric Properties of PEDOT:PSS and Tellurium-PEDOT:PSS Hybrid Composites by Simple Chemical Treatment. Sci. Rep. 6, 18805. 
122. Souto, M., Calbo, J., Mañas-Valero, S., Walsh, A., and Espallargas, G.M. (2019). Charge-transfer interactions between fullerenes and a mesoporous tetrathiafulvalene-based metal-organic framework. Beilstein J. Nanotechnol. $10,1883-1893$.

123. Kaur, R., Sen, S., Larsen, M.C., Tavares, L., Kjelstrup-Hansen, J., Ishida, M., Zieleniewska, A., Lynch, V.M., Bähring, S., Guldi, D.M., et al. (2020). Semiconducting Supramolecular Organic Frameworks Assembled from a NearInfrared Fluorescent Macrocyclic Probe and Fullerenes. J. Am. Chem. Soc. 142, 11497-11505.

124. Van Reenen, S., Scheepers, M., Van De Ruit, K., Bollen, D., and Kemerink, M. (2014). Explaining the effects of processing on the electrical properties of PEDOT:PSS. Org. Electron. 15, 3710-3714.

125. Gueye, M.N., Carella, A., Faure-Vincent, J., Demadrille, R., and Simonato, J.P. (2020). Progress in understanding structure and transport properties of PEDOTbased materials: A critical review. Prog. Mater. Sci. 108, 100616.

126. Kim, J., Jang, J.G., Hong, J.I., Kim, S.H., and Kwak, J. (2016). Sulfuric acid vapor treatment for enhancing the thermoelectric properties of PEDOT:PSS thin-films. J. Mater. Sci. Mater. Electron. 27, 6122-6127.

127. Van Der Pol, T.P.A., Keene, S.T., Saes, B.W.H., Meskers, S.C.J., Salleo, A., Van De Burgt, Y., and Janssen, R.A.J. (2019). The Mechanism of Dedoping PEDOT:PSS by Aliphatic Polyamines. J. Phys. Chem. C 123, 24328-24337.

128. Saxena, N., Keilhofer, J., Maurya, A.K., Fortunato, G., and Overbeck, J. (2018). Facile Optimization of Thermoelectric Properties in PEDOT:PSS Thin Films through Acido-Base and Redox Dedoping Using Readily Available Salts.

129. Luo, J. (2013). Materials Chemistry A Materials for energy and sustainability. 1.

130. Zapata-Arteaga, O., Dörling, B., Perevedentsev, A., Martín, J., Reparaz, J.S., and Campoy-Quiles, M. (2020). Closing the Stability-Performance Gap in Organic Thermoelectrics by Adjusting the Partial to Integer Charge Transfer $\mathbf{2 2 8} \mid \mathrm{P}$ a g e 
Ratio. Macromolecules 53, 609-620.

131. Erker, S., and Hofmann, O.T. (2019). Fractional and integer charge transfer at semiconductor/organic interfaces: The role of hybridization and metallicity. J. Phys. Chem. Lett. 10, 848-854.

132. Schwarze, M., Gaul, C., Scholz, R., Bussolotti, F., Hofacker, A., Schellhammer, K.S., Nell, B., Naab, B.D., Bao, Z., Spoltore, D., et al. (2019). Molecular parameters responsible for thermally activated transport in doped organic semiconductors. Nat. Mater. 18, 242-248.

133. Schöttner, L., Erker, S., Schlesinger, R., Koch, N., Nefedov, A., Hofmann, O.T., and Wöll, C. (2020). Doping-Induced Electron Transfer at Organic/Oxide Interfaces: Direct Evidence from Infrared Spectroscopy. J. Phys. Chem. C 124, $4511-4516$.

134. Peng, X.X., Qiao, X., Luo, S., Yao, J.A., Zhang, Y.F., and Du, F.P. (2019). Modulating carrier type for enhanced thermoelectric performance of singlewalled carbon nanotubes/polyethyleneimine composites. Polymers (Basel). 11.

135. Horike, S., Wei, Q., Kirihara, K., and Mukaida, M. (2020). Water-processable n-type doping of carbon nanotubes via charge transfer with imidazolium chloride salt. Chem. Phys. Lett. 755, 137801.

136. Fujigaya, T. (2019). Development of thermoelectric conversion materials using carbon nanotube sheets. Bull. Chem. Soc. Jpn. 92, 400-408.

137. Liu, Y., Nitschke, M., Stepien, L., Khavrus, V., Bezugly, V., and Cuniberti, G. (2019). Ammonia Plasma-Induced n-Type Doping of Semiconducting Carbon Nanotube Films: Thermoelectric Properties and Ambient Effects. ACS Appl. Mater. Interfaces 11, 21807-21814.

138. Sun, Y., Di, C.A., Xu, W., and Zhu, D. (2019). Advances in n-Type Organic Thermoelectric Materials and Devices. Adv. Electron. Mater. 5, 1-27. 
139. Massonnet, N., Carella, A., Jaudouin, O., Rannou, P., Laval, G., Celle, C., and Simonato, J.-P. (2014). Improvement of the Seebeck coefficient of PEDOT:PSS by chemical reduction combined with a novel method for its transfer using freestanding thin films. J. Mater. Chem. C 2, 1278.

140. Cho, C., Culebras, M., Wallace, K.L., Song, Y., Holder, K., Hsu, J.H., Yu, C., and Grunlan, J.C. (2016). Stable n-type thermoelectric multilayer thin films with high power factor from carbonaceous nanofillers. Nano Energy 28, 426432.

141. Kim, B., Hwang, J.U., and Kim, E. (2020). Chloride transport in conductive polymer films for an n-type thermoelectric platform. Energy Environ. Sci.

142. Peterson, K.A., Patterson, A., and Chabinyc, M.L. (2020). MATERIALS CHEMISTRY FRONTIERS Doping molecular organic semiconductors by diffusion from the vapor phase $\uparrow$.

143. Liu, J.T., Hase, H., Taylor, S., Salzmann, I., and Forgione, P. (2020). Approaching the Integer-Charge Transfer Regime in Molecularly Doped Oligothiophenes by Efficient Decarboxylative Cross-Coupling. Angew. Chemie - Int. Ed. 59, 7146-7153.

144. Dong, C.D., and Schumacher, S. (2019). Molecular Doping of PCPDT-BT Copolymers: Comparison of Molecular Complexes with and without Integer Charge Transfer. J. Phys. Chem. C 123, 30863-30870.

145. Lee, W., Kang, Y.H., Lee, J.Y., Jang, K.-S., and Cho, S.Y. (2016). Improving the thermoelectric power factor of CNT/PEDOT:PSS nanocomposite films by ethylene glycol treatment. RSC Adv. 6, 53339-53344.

146. Du, F.P., Cao, N.N., Zhang, Y.F., Fu, P., Wu, Y.G., Lin, Z.D., Shi, R., Amini, A., and Cheng, C. (2018). PEDOT:PSS/graphene quantum dots films with enhanced thermoelectric properties via strong interfacial interaction and phase separation. Sci. Rep. 8,1-12.

147. Li, J., Du, Y., Jia, R., Xu, J., and Shen, S.Z. (2017). Thermoelectric properties of flexible PEDOT: PSS/polypyrrole/paper nanocomposite films. Materials 
(Basel). 10, 6-13.

148. Zhang, B., Sun, J., Katz, H.E., Fang, F., and Opila, R.L. (2010). Promising thermoelectric properties of commercial PEDOT:PSS materials and their Bi 2 Te 3 powder composites. ACS Appl. Mater. Interfaces 2, 3170-3178.

149. Ju, H., and Kim, J. (2016). Chemically Exfoliated SnSe Nanosheets and Their SnSe/Poly(3,4-ethylenedioxythiophene):Poly(styrenesulfonate) Composite Films for Polymer Based Thermoelectric Applications. ACS Nano 10, 5730 5739.

150. Yoo, D., Lee, J.J., Park, C., Choi, H.H., and Kim, J.-H. (2016). N-type organic thermoelectric materials based on polyaniline doped with the aprotic ionic liquid 1-ethyl-3-methylimidazolium ethyl sulfate. RSC Adv. 6, 37130-37135.

151. Sundarraj, P., Maity, D., Roy, S.S., and Taylor, R.A. (2014). Recent advances in thermoelectric materials and solar thermoelectric generators-a critical review. RSC Adv. 4, 46860-46874.

152. Gaultois, M.W., Sparks, T.D., Borg, C.K.H., Seshadri, R., Bonificio, W.D., and Clarke, D.R. (2013). Data-Driven Review of Thermoelectric Materials: Performance. Chem. Mater. 25, 2911-2920.

153. He, Y., Day, T., Zhang, T., Liu, H., Shi, X., Chen, L., and Snyder, G.J. (2014). High thermoelectric performance in non-toxic earth-abundant copper sulfide. Adv. Mater. 26, 3974-3978.

154. Zhang, X., Luo, Z.-Z., Sarkar, S., Kanatzidis, M.G., and Dravid, V.P. (2018). The Effect of Spark Plasma Sintering on Microstructure Evolution in Thermoelectric Materials. Microsc. Microanal. 24, 1494-1495.

155. Ge, Z.-H., Ji, Y.-H., Qiu, Y., Chong, X., Feng, J., and He, J. (2018). Enhanced thermoelectric properties of bismuth telluride bulk achieved by telluridespilling during the spark plasma sintering process. Scr. Mater. 143, 90-93. 
156. Verma, M., Geisler, B., and Pentcheva, R. (2019). PHYSICAL REVIEW B 100 , 165126 ( 2019 ) Effect of confinement and octahedral rotations on the electronic , magnetic, and thermoelectric properties of correlated $\mathrm{Sr}$ X O 3 / SrTiO 3 ( 001 ) superlattices ( X = V , Cr, or Mn ). Phys. Rev. B 100, 165126.

157. Geisler, B., and Pentcheva, R. (2019). Inducing $\mathrm{n}$ - and $\mathrm{p}$-Type Thermoelectricity in Oxide Superlattices by Strain Tuning of Orbital-Selective Transport Resonances. Phys. Rev. Appl. 10, 1.

158. Thermoelectric Properties of the cubic AgPb 10 SbTe 12 (2004). 793, 8-13.

159. Yamasaka, S., Nakamura, Y., Ueda, T., Takeuchi, S., and Sakai, A. (2015). Phonon transport control by nanoarchitecture including epitaxial Ge nanodots for Si-based thermoelectric materials. Sci. Rep. 5, 1-9.

160. Kim, J.H., Jung, Y.C., Suh, S.H., and Kim, J.S. (2006). MOCVD of Bi 2Te 3 and $\mathrm{Sb} 2 \mathrm{Te} 3$ on GaAs substrates for thin-film thermoelectric applications. J. Nanosci. Nanotechnol. 6, 3325-3328.

161. Banerjee, A.N., Maity, R., Ghosh, P.K., and Chattopadhyay, K.K. (2005). Thermoelectric properties and electrical characteristics of sputter-deposited pCuAlO2 thin films. Thin Solid Films 474, 261-266.

162. Ahmad, K., Wan, C., Al-Eshaikh, M.A., and Kadachi, A.N. (2019). Enhanced thermoelectric performance of Bi2Te3 based graphene nanocomposites. Appl. Surf. Sci. 474, 2-8.

163. Mori, R., Mayuzumi, Y., Yamaguchi, M., Kobayashi, A., Seki, Y., and Takashiri, M. (2019). Improved thermoelectric properties of solvothermally synthesized Bi2Te3 nanoplate films with homogeneous interconnections using Bi2Te3 electrodeposited layers. J. Alloys Compd., 152901.

164. Im, H.J., Koo, B., Kim, M.-S., and Lee, J.E. (2019). Solvothermal synthesis of $\mathrm{Sb} 2 \mathrm{Te} 3$ nanoplates under various synthetic conditions and their thermoelectric properties. Appl. Surf. Sci. 475, 510-514.

165. Lin, F.-H., and Liu, C.-J. (2019). A simple energy-saving aqueous synthesis of 
Bi2Te3 nanocomposites yielding relatively high thermoelectric power factors. Ceram. Int. 45, 9397-9400.

166. Zhang, Q., Sun, Y., Xu, W., and Zhu, D. (2014). Organic Thermoelectric Materials : Emerging Green Energy Materials Converting Heat to Electricity Directly and Effi ciently.

167. Di, C., Xu, W., and Zhu, D. (2016). Organic thermoelectrics for green energy EXPERIENCING RAPID. 1-3.

168. Ito, M., Nagira, T., and Hara, S. (2006). Thermoelectric properties of NaxCo2O4 with rare-earth metals doping prepared by polymerized complex method. J. Alloys Compd. 408-412, 1217-1221.

169. Born, H.J., Legvold, S., Spedding, F.H., and Spectra, L.F. (1988). LowTemperature Thermoelectric Power of the RareEarth Metals. 2543.

170. Moriarty, G.P., De, S., King, P.J., Khan, U., Via, M., King, J.A., Coleman, J.N., and Grunlan, J.C. (2013). Thermoelectric Behavior of Organic Thin Film Nanocomposites. 119-123.

171. Yue, R., and Xu, J. (2012). Poly(3,4-ethylenedioxythiophene) as promising organic thermoelectric materials: A mini-review. Synth. Met. 162, 912-917.

172. Yan, H., Sada, N., and Toshima, N. (2002). Thermal transporting properties of electrically conductive polyaniline films as organic thermoelectric materials. J. Therm. Anal. Calorim. 69, 881-887.

173. Ren, X., Yang, F., Gao, X., Cheng, S., Zhang, X., Dong, H., and Hu, W. (2018). Organic Field-Effect Transistor for Energy-Related Applications: Low-PowerConsumption Devices, Near-Infrared Phototransistors, and Organic Thermoelectric Devices. Adv. Energy Mater. 8, 1-27.

174. Un, H., Gregory, S.A., Mohapatra, S.K., Xiong, M., Longhi, E., Lu, Y., Rigin, S., Jhulki, S., Yang, C., Timofeeva, T. V, et al. (2019). Understanding the 
Effects of Molecular Dopant on n-Type Organic Thermoelectric Properties. $1900817,1-10$.

175. Sun, Y., Sheng, P., Di, C., Jiao, F., Xu, W., Qiu, D., and Zhu, D. (2012). Organic Thermoelectric Materials and Devices Based on $\mathrm{p}$ - and n -Type Poly ( metal 1 , 1, 2, 2-ethenetetrathiolate ) s. 932-937.

176. Sanchez-Sanchez, A., del Agua, I., Malliaras, G.G., and Mecerreyes, D. (2019). Chapter 6 - Conductive Poly(3,4-Ethylenedioxythiophene) (PEDOT)-Based Polymers and Their Applications in Bioelectronics. In Woodhead Publishing in Materials, M. R. Aguilar and J. B. T.-S. P. and their A. (Second E. San Román, eds. (Woodhead Publishing), pp. 191-218.

177. Bruijnaers, B.J., Schiepers, E., Weijtens, C.H.L., and Meskers, S.C.J. (2018). The e ff ect of oxygen on the e ffi ciency of planar $p-i-n$ metal halide perovskite solar cells with a PEDOT : PSS hole transport layer $\uparrow .6882-6890$.

178. Huang, X., Wang, K., Yi, C., Meng, T., and Gong, X. (2016). Effi cient Perovskite Hybrid Solar Cells by Highly Electrical Conductive PEDOT : PSS Hole Transport Layer. 1-8.

179. Chou, T.R., Chen, S.H., Chiang, Y. Te, Lin, Y.T., and Chao, C.Y. (2015). Highly Conductive PEDOT:PSS Film by Post-Treatment with Dimethyl Sulfoxide for ITO-Free Liquid Crystal Display. Mol. Cryst. Liq. Cryst. 612, $201-210$.

180. Singh, R., Tharion, J., Murugan, S., and Kumar, A. (2016). ITO-Free SolutionProcessed Flexible Electrochromic Devices Based on PEDOT: PSS as Transparent Conducting Electrode.

181. Zhang, X., Wu, J., Wang, J., Zhang, J., Yang, Q., Fu, Y., and Xie, Z. (2016). Highly conductive PEDOT:PSS transparent electrode prepared by a post-spinrinsing method for efficient ITO-free polymer solar cells. Sol. Energy Mater. Sol. Cells 144, 143-149.

182. Singh, R., Tharion, J., Murugan, S., and Kumar, A. (2017). ITO-Free SolutionProcessed Flexible Electrochromic Devices Based on PEDOT:PSS as 
Transparent Conducting Electrode. ACS Appl. Mater. Interfaces 9, 1942719435.

183. Liu, S., Yu, H., Zhang, Q., Qin, F., Zhang, X., Zhang, L., and Xie, W. (2019). Efficient ITO-free organic light-emitting devices with dual-functional PSS-rich PEDOT:PSS electrode by enhancing carrier balance. J. Mater. Chem. C 7, $5426-5432$.

184. Pan, Q., Liu, Q., Yang, Y., and Tian, D. (2018). Abnormal Capacitance Increasing at Elevated Temperature in Tantalum Capacitors with PEDOT : PSS Electrodes. 2018.

185. Zhang, M., Yu, X., Ma, H., Du, W., Qu, L., Li, C., and Shi, G. (2018). Robust graphene composite films for multifunctional electrochemical capacitors with an ultrawide range of areal mass loading toward high-rate frequency response and ultrahigh specific capacitance. Energy Environ. Sci. 11, 559-565.

186. Wen, Z., Yang, Y., Sun, N., Li, G., Liu, Y., Chen, C., and Shi, J. (2018). A Wrinkled PEDOT : PSS Film Based Stretchable and Transparent Triboelectric Nanogenerator for Wearable Energy Harvesters and Active Motion Sensors. $1803684,1-8$.

187. Birgerson, J., Crispin, X., Greczynski, G., Osikowicz, W., Gon, A.W.D. Van Der, Salaneck, W.R., and Fahlman, M. (2003). The effects of solvents on the morphology and sheet resistance acid ( PEDOT - PSS ) films. 139, 1-10.

188. Shi, H., Liu, C., Jiang, Q., and Xu, J. (2015). Effective Approaches to Improve the Electrical Conductivity of PEDOT : PSS : A Review. 1-16.

189. Treatment, P. (2018). Tailoring Characteristics of PEDOT: PSS Coated on Glass and Plastics by Ultrasonic Substrate Vibration Post Treatment.

190. Fan, X., Nie, W., Tsai, H., Wang, N., Huang, H., and Cheng, Y. (2019). PEDOT: PSS for Flexible and Stretchable Electronics: Modifications, Strategies, and Applications. 1900813. 
191. Liu, J., Wang, X., Li, D., Coates, N.E., Segalman, R.A., and Cahill, D.G. (2015). Thermal Conductivity and Elastic Constants of PEDOT:PSS with High Electrical Conductivity. Macromolecules 48, 585-591.

192. Zhu, X.-H., Peng, J., Cao, Y., and Roncali, J. (2011). Solution-processable single-material molecular emitters for organic light-emitting devices. Chem. Soc. Rev. 40, 3509-3524.

193. Lee, S.H., Park, H., Son, W., Choi, H.H., and Kim, J.H. (2014). Novel solutionprocessable $\{$,$\} dedoped semiconductors for application in thermoelectric$ devices. J. Mater. Chem. A 2, 13380-13387.

194. Morris, J.D., and Payne, C.K. (2014). Tuning PEDOT:PSS conductivity with iron oxidants. Org. Electron. 15, 1707-1710.

195. Cai, W., Österberg, T., Jafari, M.J., Musumeci, C., Wang, C., Zuo, G., Yin, X., Luo, X., Johansson, J., Kemerink, M., et al. (2020). Dedoping-induced interfacial instability of poly(ethylene imine)s-treated PEDOT:PSS as a lowwork-function electrode. J. Mater. Chem. C 8, 328-336.

196. Crispin, X., Marciniak, S., Osikowicz, W., Zotti, G., Gon, a W.D.V. a N.D.E.R., Louwet, F., Fahlman, M., Groenendaal, L., Schryver, F.D.E., and Salaneck, W.R. (2003). Stability of Poly ( 3 , 4-ethylene dioxythiophene )- Poly ( styrene sulfonate ): A Photoelectron Spectroscopy Study. Polymer (Guildf). $41,2561-2583$.

197. Kee, S., Kim, H., Paleti, S.H.K., El Labban, A., Neophytou, M., Emwas, A.-H., Alshareef, H.N., and Baran, D. (2019). Highly Stretchable and Air-Stable PEDOT:PSS/Ionic Liquid Composites for Efficient Organic Thermoelectrics. Chem. Mater., acs.chemmater.9b00819.

198. Nguyen, T.P., and Vos, S.A. De (2004). An investigation into the effect of chemical and thermal treatments on the structural changes of poly ( 3 , 4ethylenedioxythiophene )/ polystyrenesulfonate and consequences on its use on indium tin oxide substrates. 221, 330-339.

199. Wang, Y., Cai, K., and Yao, X. (2011). Facile Fabrication and Thermoelectric $\mathbf{2 3 6} \mid \mathrm{P}$ a g e 
Properties of PbTe-Modified. 1163-1166.

200. Rahman, A.A.A., Ali Umar, A., and Othman, M.H.U. (2015). Effect of bismuth telluride concentration on the thermoelectric properties of PEDOT:PSSglycerol organic films. Phys. E Low-Dimensional Syst. Nanostructures 66, 293-298.

201. Zhou, Z., Yang, J., Jiang, Q., Li, W., Luo, Y., Hou, Y., Zhou, S., and Li, X. (2016). Large improvement of device performance by a synergistic effect of photovoltaics and thermoelectrics. Nano Energy 22, 120-128.

202. Ha, S.R., Park, S., Oh, J.T., Kim, D.H., Cho, S., Bae, S.Y., Kang, D.-W., Kim, J.-M., and Choi, H. (2018). Water-resistant PEDOT:PSS hole transport layers by incorporating a photo-crosslinking agent for high-performance perovskite and polymer solar cells. Nanoscale 10, 13187-13193.

203. Park, M.U., Lee, S.M., and Chung, D. (2019). Model system of cross-linked PEDOT:PSS adaptable to an application for an electrode with enhanced water stability. Synth. Met. 258, 116195.

204. Ni, D., Song, H., Chen, Y., and Cai, K. (2019). Significantly enhanced thermoelectric performance of flexible PEDOT nanowire film via coating Te nanostructures. J. Mater.

205. Ye, G., Xu, J., Ma, X., Zhou, Q., Li, D., Zuo, Y., Lv, L., Zhou, W., and Duan, X. (2017). One-step electrodeposition of free-standing flexible conducting PEDOT derivative film and its electrochemical capacitive and thermoelectric performance. Electrochim. Acta 224, 125-132.

206. Jiang, Q., Liu, C., Song, H., Xu, J., Mo, D., Shi, H., Wang, Z., Jiang, F., Lu, B., and Zhu, Z. (2014). Free-standing PEDOT: PSS film as electrode for the electrodeposition of bismuth telluride and its thermoelectric performance. Int. J. Electrochem. Sci. 9, 7540-7551.

207. Huang, J., Miller, P.F., Wilson, J.S., De Mello, A.J., De Mello, J.C., and $\mathbf{2 3 7} \mid \mathrm{P}$ a g e 
Bradley, D.D.C. (2005). Investigation of the effects of doping and postdeposition treatments on the conductivity, morphology, and work function of poly(3,4- ethylenedioxythiophene)/poly(styrene sulfonate) films. Adv. Funct. Mater. 15, 290-296.

208. Blackburn, J.L., Ferguson, A.J., Cho, C., and Grunlan, J.C. (2018). Thermoelectric Materials: Carbon-Nanotube-Based Thermoelectric Materials and Devices (Adv. Mater. 11/2018). Adv. Mater. 30, 1870072.

209. Park, H., Lee, S.H., Kim, F.S., Choi, H.H., Cheong, I.W., and Kim, J.H. (2014). Enhanced thermoelectric properties of PEDOT:PSS nanofilms by a chemical dedoping process. J. Mater. Chem. A 2, 6532-6539.

210. Mengistie, D.A., Chen, C.H., Boopathi, K.M., Pranoto, F.W., Li, L.J., and Chu, C.W. (2015). Enhanced thermoelectric performance of PEDOT:PSS flexible bulky papers by treatment with secondary dopants. ACS Appl. Mater. Interfaces 7, 94-100.

211. Kim, G.-H., Shao, L., Zhang, K., and Pipe, K.P. (2013). Engineered doping of organic semiconductors for enhanced thermoelectric efficiency. Nat. Mater. 12, $719-723$.

212. Luo, J., Billep, D., Waechtler, T., Otto, T., Toader, M., Gordan, O., Sheremet, E., Martin, J., Hietschold, M., Zahn, D.R.T., et al. (2013). Enhancement of the thermoelectric properties of PEDOT:PSS thin films by post-treatment. J. Mater. Chem. A 1, 7576-7583.

213. Kim, G.-H., Shao, L., Zhang, K., and Pipe, K.P. (2013). s. Nat. Mater. 12, 719 23.

214. Kumar, S.R.S., Kurra, N., and Alshareef, H.N. (2016). Enhanced high temperature thermoelectric response of sulphuric acid treated conducting polymer thin films. J. Mater. Chem. C 4, 215-221.

215. Yang, L., Gordon, M.P., Menon, A.K., Bruefach, A., Haas, K., Scott, M.C., Prasher, R.S., and Urban, J.J. (2021). Decoupling electron and phonon transport in single-nanowire hybrid materials for high-performance thermoelectrics. Sci. 
Adv. 7, eabe6000.

216. Döbbelin, M., Marcilla, R., Salsamendi, M., Pozo-Gonzalo, C., Carrasco, P.M., Pomposo, J.A., and Mecerreyes, D. (2007). Influence of Ionic Liquids on the Electrical Conductivity and Morphology of PEDOT:PSS Films. Chem. Mater. $19,2147-2149$.

217. Kee, S., Kim, N., Kim, B.S., Park, S., Jang, Y.H., Lee, S.H., Kim, J., Kim, J., Kwon, S., and Lee, K. (2016). Controlling Molecular Ordering in Aqueous Conducting Polymers Using Ionic Liquids. 8625-8631.

218. Kee, S., Kim, H., Paleti, S.H.K., El Labban, A., Neophytou, M., Emwas, A.-H., Alshareef, H.N., and Baran, D. (2019). Highly Stretchable and Air-Stable PEDOT:PSS/Ionic Liquid Composites for Efficient Organic Thermoelectrics. Chem. Mater., acs.chemmater.9b00819.

219. Meng, Q., Jiang, Q., Cai, K., and Chen, L. (2019). Preparation and thermoelectric properties of PEDOT:PSS coated Te nanorod/PEDOT:PSS composite films. Org. Electron. 64, 79-85.

220. Kumar, A., Battabyal, M., Chauhan, A., Suresh, G., Gopalan, R., kumar, N.V.R., and Satapathy, D.K. (2019). Charge transport mechanism and thermoelectric behavior in Te:( $\{\mathrm{PEDOT}\}:\{\mathrm{PSS}\})$ polymer composites. Mater. Res. Express 6, 115302.

221. Ju, H., and Kim, J. (2016). Fabrication of conductive polymer / inorganic nanoparticles composite films: PEDOT: PSS with exfoliated tin selenide nanosheets for polymer-based thermoelectric devices. Chem. Eng. J. 297, 6673.

222. Burton, M.Rt.F.T.S. (SnSe) T.G.E.U.T.C., Liu, T., McGettrick, J., Mehraban, S., Baker, J., Pockett, A., Watson, T., Fenwick, O., and Carnie, M.J. (2018). Thin Film Tin Selenide (SnSe) Thermoelectric Generators Exhibiting Ultralow Thermal Conductivity. Adv. Mater. 30, 1801357. 
223. Sist, M., Zhang, J., and Brummerstedt Iversen, B. (2016). Crystal structure and phase transition of thermoelectric SnSe. Acta Crystallogr. Sect. B 72, 310-316.

224. Bae, E.J., Kang, Y.H., Jang, K.-S., and Cho, S.Y. (2016). Enhancement of Thermoelectric Properties of PEDOT:PSS and Tellurium-PEDOT:PSS Hybrid Composites by Simple Chemical Treatment. Sci. Rep. 6, 18805.

225. Kim, C., Hong, J., and Park, J. (2019). Synthesis and Thermoelectric Properties of Selenium Nanoparticles Coated with PEDOT : PSS.

226. Wang, X., Liu, P., Jiang, Q., Zhou, W., Xu, J., Liu, J., Jia, Y., Duan, X., Liu, Y., Du, Y., et al. (2019). Efficient DMSO-Vapor Annealing for Enhancing Thermoelectric Performance of PEDOT:PSS-Based Aerogel. ACS Appl. Mater. Interfaces 11, 2408-2417.

227. Kim, W.S., Anoop, G., Jeong, I.-S., Lee, H.J., Kim, H. Bin, Kim, S.H., Goo, G.W., Lee, H., Lee, H.J., Kim, C., et al. (2020). Feasible tuning of barrier energy in PEDOT:PSS/Bi2Te3 nanowires-based thermoelectric nanocomposite thin films through polar solvent vapor annealing. Nano Energy 67, 104207.

228. Martins, P., and Antunes, P. (2020). Overview on thermoactive materials, simulations and applications. 925-946.

229. Song, H., Meng, Q., Lu, Y., and Cai, K. (2019). Improving the thermoelectric power factor of CNT/PEDOT:PSS nanocomposite films by ethylene glycol treatment. Adv. Electron. Mater. 5, 1800822.

230. Ju, H., Park, D., Kim, K., and Kim, J. (2019). Exfoliated Sn-Se-Te based nanosheets and their flexible thermoelectric composites with poly(3,4ethylenedioxythiophene):poly(styrenesulfonate) fabricated by solution processing. Org. Electron. 71, 131-135.

231. Ju, H., Park, D., Kim, K., and Kim, J. (2019). Chemical exfoliation of SnSe 1 e $\mathrm{x}$ Te $\mathrm{x}$ nanosheets with conductive PEDOT : PSS for fl exible thermoelectric composite fi lms. 792, 638-643.

232. Li, Y., Du, Y., Dou, Y., Cai, K., and Xu, J. (2017). PEDOT-based $\mathbf{2 4 0} \mid \mathrm{P}$ a g e 
thermoelectric nanocomposites - A mini-review. Synth. Met. 226, 119-128.

233. Sugahara, T., Ekubaru, Y., Nong, N. Van, Kagami, N., Ohata, K., Hung, L.T., Okajima, M., Nambu, S., and Suganuma, K. (2019). Fabrication with Semiconductor Packaging Technologies and Characterization of a Large-Scale Flexible Thermoelectric Module. Adv. Mater. Technol. 4, 1800556.

234. Kim, J.Y., Lee, W., Kang, Y.H., Cho, S.Y., and Jang, K.S. (2018). Wet-spinning and post-treatment of CNT/PEDOT:PSS composites for use in organic fiberbased thermoelectric generators. Carbon N. Y. 133, 293-299.

235. Wang, H., Hsu, J., Yi, S., Kim, S.L., Choi, K., and Yang, G. (2015). Thermally Driven Large N-Type Voltage Responses from Hybrids of Carbon Nanotubes and Poly ( 3 , 4- ethylenedioxythiophene ) with Tetrakis (dimethylamino ) ethylene COMMUNICATION. 6855-6861.

236. Lee, B.H. Electronic Applications of Graphene / PVDF-TrFE stack.

237. da Cunha Rodrigues, G., Zelenovskiy, P., Romanyuk, K., Luchkin, S., Kopelevich, Y., and Kholkin, A. (2015). Strong piezoelectricity in single-layer graphene deposited on $\mathrm{SiO} 2$ grating substrates. Nat. Commun. 6, 7572.

238. Acik, M., and Darling, S.B. (2016). Graphene in perovskite solar cells: device design, characterization and implementation. J. Mater. Chem. A 4, 6185-6235.

239. Sanli, A., Müller, C., Kanoun, O., Elibol, C., and Wagner, M.F.X. (2016). Piezoresistive characterization of multi-walled carbon nanotube-epoxy based flexible strain sensitive films by impedance spectroscopy. Compos. Sci. Technol. 122, 18-26.

240. Mahmoud, L., Alhwarai, M., Samad, Y.A., Mohammad, B., Laio, K., and Elnaggar, I. (2015). Characterization of a Graphene-Based Thermoelectric Generator Using a Cost-Effective Fabrication Process. Energy Procedia 75, 615-620. 
241. Kim, Y.-J., Kim, Y., Novoselov, K., and Hong, B.H. (2015). Engineering electrical properties of graphene: chemical approaches. 2D Mater. 2, 042001.

242. Serov, A., Ong, Z., and Pop, E. (2013). Effect of grain boundaries on thermal transport in graphene. Appl. Phys. Lett. 102, 033104.

243. Wang, Y., Song, Z., and Xu, Z. (2014). Characterizing phonon thermal conduction in polycrystalline graphene. J. Mater. Res. 29, 362-372.

244. Liu, H.K., Lin, Y., and Luo, S.N. (2014). Grain boundary energy and grain size dependences of thermal conductivity of polycrystalline graphene. J. Phys. Chem. C 118, 24797-24802.

245. Ishikawa, K., Okada, A., and Itoh, T. (2007). Influence of Fullerene Addition on Thermoelectric Properties of Non-filled / Filled Cobalt-Skutterudite Compounds. 07, 151-154.

246. Zhang, K., Zhang, Y., and Wang, S. (2013). Enhancing thermoelectric properties of organic composites through hierarchical nanostructures. Sci. Rep. $3,3448-1-7$.

247. Yoo, D., Kim, J., and Kim, J.H. (2014). Direct synthesis of highly conductive poly(3,4-ethylenedioxythiophene):Poly(4-styrenesulfonate)

(PEDOT:PSS)/graphene composites and their applications in energy harvesting systems. Nano Res. 7, 717-730.

248. Yoo, D., Kim, J., Lee, S.H., Cho, W., Choi, H.H., Kim, F.S., and Kim, J.H. (2015). hybridization of PEDOT: PSS on the power factor of polymer thermoelectric energy conversion. J. Mater. Chem. A Mater. energy Sustain. 3, 6526-6533.

249. Li, X., Liang, L., Yang, M., Chen, G., and Guo, C.-Y. (2016). Poly(3,4ethylenedioxythiophene)/graphene/carbon nanotube ternary composites with improved thermoelectric performance. Org. Electron. 38, 200-204.

250. Nakai, Y., Honda, K., Yanagi, K., Kataura, H., Kato, T., Yamamoto, T., and Maniwa, Y. (1882). Giant Seebeck coefficient in semiconducting single-wall 
carbon nanotube film. 025103, 1-16.

251. By, P. World' s largest Science, Technology \& Medicine Open Access book publisher Phonon Scattering and Electron Transport in Single Wall Carbon Nanotube.

252. Benchirouf, A., Palaniyappan, S., Ramalingame, R., Raghunandan, P., Jagemann, T., Müller, C., Hietschold, M., and Kanoun, O. (2016). Electrical properties of multi-walled carbon nanotubes/PEDOT:PSS nanocomposites thin films under temperature and humidity effects. Sensors Actuators, B Chem. 224, 344-350.

253. Yu, C., Choi, K., Yin, L., and Grunlan, J.C. (2013). Correction to Light-Weight Flexible Carbon Nanotube Based Organic Composites with Large Thermoelectric Power Factors. ACS Nano 7, 9506-9506.

254. Toshima, N., Oshima, K., Anno, H., Nishinaka, T., Ichikawa, S., Iwata, A., and Shiraishi, Y. (2015). Novel Hybrid Organic Thermoelectric Materials:ThreeComponent Hybrid Films Consisting of a Nanoparticle Polymer Complex, Carbon Nanotubes, and Vinyl Polymer. Adv. Mater. 27, 2246-2251.

255. Suemori, K., Watanabe, Y., and Hoshino, S. (2016). Increase in thermoelectric power factor of carbon-nanotube films after addition of polystyrene. Org. Electron. physics, Mater. Appl. 28, 135-138.

256. Liang, L., Chen, G., and Guo, C.Y. (2016). Enhanced thermoelectric performance by self-assembled layered morphology of polypyrrole nanowire/single-walled carbon nanotube composites. Compos. Sci. Technol. $129,130-136$.

257. Zhang, H.-L., Li, J.-F., Zhang, B.-P., Yao, K.-F., Liu, W.-S., and Wang, H. (2007). Electrical and thermal properties of carbon nanotube bulk materials: Experimental studies for the 328-958K temperature range. Phys. Rev. B 75, 19. 
258. Small, J.P., Perez, K.M., and Kim, P. (2003). Modulation of Thermoelectric Power of Individual Carbon Nanotubes. Phys. Rev. Lett. 91, 256801.

259. Arnold, M.S., Green, A.A., Hulvat, J.F., Stupp, S.I., and Hersam, M.C. (2006). Sorting carbon nanotubes by electronic structure using density differentiation. Nat. Nanotechnol. 1, 60-65.

260. Yanagi, K., Miyata, Y., and Kataura, H. (2008). Optical and conductive characteristics of metallic single-wall carbon nanotubes with three basic colors; cyan, magenta, and yellow. Appl. Phys. Express 1, 0340031-0340033.

261. Kuwahara, S., Kuwahara, Y., and Shinohara, H. (2014). Quantitative analysis of isolated single-wall carbon nanotubes with their molar absorbance coefficients. J. Nanomater. 2014, 1-8.

262. Lee, M.M., Teuscher, J., Miyasaka, T., Murakami, T.N., and Snaith, H.J. (2012). Efficient hybrid solar cells based on meso-superstructured organometal halide perovskites. Science 338, 643-7.

263. Kim, H.-S., Lee, C.-R., Im, J.-H., Lee, K.-B., Moehl, T., Marchioro, A., Moon, S.-J., Humphry-Baker, R., Yum, J.-H., Moser, J.E., et al. (2012). Lead Iodide Perovskite Sensitized All-Solid-State Submicron Thin Film Mesoscopic Solar Cell with Efficiency Exceeding 9\%. Scientific 2, 1-7.

264. Carnie, M.J., Charbonneau, C., Davies, M.L., Troughton, J., Watson, T.M., Wojciechowski, K., Snaith, H., and Worsley, D.A. (2013). A one-step low temperature processing route for organolead halide perovskite solar cells. Chem. Commun. 49, 7893-7895.

265. Stoumpos, C.C., Malliakas, C.D., and Kanatzidis, M.G. (2013). Semiconducting tin and lead iodide perovskites with organic cations: Phase transitions, high mobilities, and near-infrared photoluminescent properties. Inorg. Chem. 52, 9019-9038.

266. He, Y., and Galli, G. (2014). Perovskites for Solar Thermoelectric Applications: A First Principle Study of CH 3 NH 3 AI $3(\mathrm{~A}=\mathrm{Pb}$ and $\mathrm{Sn})$. Chem. Mater. 26, 5394-5400. 
267. Hao, F., Stoumpos, C.C., Cao, D.H., Chang, R.P.H., and Kanatzidis, M.G. (2014). Lead-free solid-state organic-inorganic halide perovskite solar cells. Nat. Photonics 8, 489-494.

268. Hébert, S., Flahaut, D., Martin, C., Lemonnier, S., Noudem, J., Goupil, C., Maignan, A., and Hejtmanek, J. (2007). Thermoelectric properties of perovskites: Sign change of the Seebeck coefficient and high temperature properties. Prog. Solid State Chem. 35, 457-467.

269. Park, K.-T., Shin, S.-M., Tazebay, A.S., Um, H.-D., Jung, J.-Y., Jee, S.-W., Oh, M.-W., Park, S.-D., Yoo, B., Yu, C., et al. (2013). Lossless hybridization between photovoltaic and thermoelectric devices. Sci. Rep. 3, 2123.

270. Sark, W.G.J.H.M. van (2011). Enhancing thermoelectric properties of organic composites. Appl. EnergyFeasibility Photovolt. - Thermoelectr. hybrid Modul. $88,2785-2790$.

271. Lee, J.J., Yoo, D., Park, C., Choi, H.H., and Kim, J.H. (2016). All organic-based solar cell and thermoelectric generator hybrid device system using highly conductive PEDOT:PSS film as organic thermoelectric generator. Sol. Energy 134, 479-483.

272. Hwang, S., Potscavage, W.J., Nakamichi, R., and Adachi, C. (2016). Processing and doping of thick polymer active layers for flexible organic thermoelectric modules. Org. Electron. physics, Mater. Appl. 31, 31-40.

273. Liu, T., Zhao, X., Li, J., Liu, Z., Liscio, F., Milita, S., Schroeder, B.C., and Fenwick, O. (2019). Enhanced control of self-doping in halide perovskites for improved thermoelectric performance. Nat. Commun. 10, 1-9.

274. Dey, A., Hadavale, S., Khan, M.A.S., More, P., Khanna, P.K., Sikder, A.K., and Chattopadhyay, S. (2015). Polymer based graphene/titanium dioxide nanocomposite (GTNC): an emerging and efficient thermoelectric material. Dalt. Trans., 19248-19255. 
275. Wang, H., and Yu, C. (2019). Organic Thermoelectrics: Materials Preparation, Performance Optimization, and Device Integration. Joule 3, 53-80.

276. Xu, S., Hong, M., Shi, X.-L., Wang, Y., Ge, L., Bai, Y., Wang, L., Dargusch, M., Zou, J., and Chen, Z.-G. (2019). High-Performance PEDOT:PSS Flexible Thermoelectric Materials and Their Devices by Triple Post-Treatments. Chem. Mater. 31, 5238-5244.

277. Chiu, M.-H., Fan, K.-H., Hsu, H.-C., and Li, W.-Y. (2020). 1-to-100-micron surface height full-field 3D topography by use of the external reflectance versus height conversion method. OSA Contin. 3, 1154.

278. Pereira, T., Potgieter, J., Kennedy, J. V., Mukhtar, A., and Fry, M. (2019). The effect of reheating layers in Metal Additive Manufacturing on the external surface finish of a printed part. Proc. 2018 25th Int. Conf. Mechatronics Mach. Vis. Pract. M2VIP 2018, 8-13.

279. Schaubroeck, D., De Smet, J., Willems, W., Cools, P., De Geyter, N., Morent, R., De Smet, H., and Van Steenbeerge, G. (2016). Surface analysis of the selective excimer laser patterning of a thin PEDOT:PSS film on flexible polymer films. Appl. Surf. Sci. 376, 151-160.

280. Ezra, L., O’Dell, Z.J., Hui, J., and Riley, K.R. (2020). Emerging investigator series: quantifying silver nanoparticle aggregation kinetics in real-time using particle impact voltammetry coupled with UV-vis spectroscopy. Environ. Sci. Nano 7, 2509-2521.

281. Liu, S., Li, H., and He, C. (2019). Simultaneous enhancement of electrical conductivity and seebeck coefficient in organic thermoelectric SWNT/PEDOT:PSS nanocomposites. Carbon N. Y. 149, 25-32.

282. Zhou, W., Begum, S., Wang, Z., Krolla, P., Wagner, D., Bräse, S., Wöll, C., and Tsotsalas, M. (2018). High Antimicrobial Activity of Metal-Organic Framework-Templated Porphyrin Polymer Thin Films. ACS Appl. Mater. Interfaces 10, 1528-1533.

283. Costa, J.C.S., Taveira, R.J.S., Lima, C.F.R.A.C., Mendes, A., and Santos, $246 \mid$ P a g e 
L.M.N.B.F. (2016). Optical band gaps of organic semiconductor materials. Opt. Mater. (Amst). 58, 51-60.

284. Oh, J.S., Szili, E.J., Ogawa, K., Short, R.D., Ito, M., Furuta, H., and Hatta, A. (2018). UV-vis spectroscopy study of plasma-activated water: Dependence of the chemical composition on plasma exposure time and treatment distance. Jpn. J. Appl. Phys. 57.

285. Grasse, E.K., Torcasio, M.H., and Smith, A.W. (2016). Teaching UV-Vis Spectroscopy with a 3D-Printable Smartphone Spectrophotometer. J. Chem. Educ. 93, 146-151.

286. Rocha, F.S., Gomes, A.J., Lunardi, C.N., Kaliaguine, S., and Patience, G.S. (2018). Experimental methods in chemical engineering: Ultraviolet visible spectroscopy-UV-Vis. Can. J. Chem. Eng. 96, 2512-2517.

287. Zhang, L., Yang, K., Chen, R., Zhou, Y., Chen, S., Zheng, Y., Li, M., Xu, C., Tang, X., Zang, Z., et al. (2020). The Role of Mineral Acid Doping of PEDOT:PSS and Its Application in Organic Photovoltaics. Adv. Electron. Mater. 6, 1900648.

288. Kalagi, S.S., and Patil, P.S. (2016). Secondary electrochemical doping level effects on polaron and bipolaron bands evolution and interband transition energy from absorbance spectra of PEDOT: PSS thin films. Synth. Met. 220, 661-666.

289. Cruz-Cruz, I., Reyes-Reyes, M., Aguilar-Frutis, M.A., Rodriguez, A.G., and López-Sandoval, R. (2010). Study of the effect of DMSO concentration on the thickness of the PSS insulating barrier in PEDOT:PSS thin films. Synth. Met. $160,1501-1506$.

290. Selvakumar, N., Karthik, G., Jayaraj, S., and Barshilia, H.C. (2021). Sprayable PEDOT:PSS based spectrally selective coating for solar energy harvesting. Sol. Energy Mater. Sol. Cells 221, 110906. 
291. Cordero, E., Latka, I., Matthäus, C., Schie, I.W., and Popp, J. (2018). In-vivo Raman spectroscopy: from basics to applications. J. Biomed. Opt. 23, 1-23.

292. Rostron, P., Gaber, S., and Gaber, D. (2016). Raman Spectroscopy, Review.

293. Bumbrah, G.S., and Sharma, R.M. (2016). Raman spectroscopy - Basic principle, instrumentation and selected applications for the characterization of drugs of abuse. Egypt. J. Forensic Sci. 6, 209-215.

294. Li, C., Ye, Z., Xu, Y., and Bell, S.E.J. (2020). An overview of therapeutic anticancer drug monitoring based on surface enhanced (resonance) Raman spectroscopy (SE(R)RS). Analyst.

295. Hong, S., Lee, D.M., Park, M., Wee, J.H., Jeong, H.S., Ku, B.C., Yang, C.M., Lee, D.S., Terrones, M., Kim, Y.A., et al. (2020). Controlled synthesis of Ntype single-walled carbon nanotubes with $100 \%$ of quaternary nitrogen. Carbon N. Y. $167,881-887$.

296. Zeng, W., Tao, X.M., Lin, S., Lee, C., Shi, D., Lam, K. ho, Huang, B., Wang, Q., and Zhao, Y. (2018). Defect-engineered reduced graphene oxide sheets with high electric conductivity and controlled thermal conductivity for soft and flexible wearable thermoelectric generators. Nano Energy 54, 163-174.

297. Chernyak, S.A., Ivanov, A.S., Maslakov, K.I., Egorov, A. V, Shen, Z., Savilov, S.S., and Lunin, V. V (2017). Oxidation $\{$,$\} defunctionalization and catalyst life$ cycle of carbon nanotubes: a Raman spectroscopy view. Phys. Chem. Chem. Phys. 19, 2276-2285.

298. Wang, X., Li, M., Feng, G., and Ge, M. (2020). On the mechanism of conductivity enhancement in PEDOT:PSS/PVA blend fiber induced by UVlight irradiation. Appl. Phys. A 126, 184.

299. Hwang, J.S., Oh, T.H., Kim, S.H., Han, S.S., Lee, S.J., Lee, S.G., Lee, Y.J., and Jang, S.S. (2015). Effect of solvent on electrical conductivity and gas sensitivity of PEDOT: PSS polymer composite films. J. Appl. Polym. Sci. 132, 2-7.

300. Lenz, A., Kariis, H., Pohl, A., Persson, P., and Ojamäe, L. (2011). The 
electronic structure and reflectivity of PEDOT : PSS from density functional theory. $44-51$.

301. Ye, K., Li, K., Lu, Y., Guo, Z., Ni, N., Liu, H., Huang, Y., Ji, H., and Wang, P. (2019). An overview of advanced methods for the characterization of oxygen vacancies in materials. TrAC Trends Anal. Chem. 116, 102-108.

302. Shard, A.G. (2020). Chapter 4.3.1 - X-ray photoelectron spectroscopy. In Characterization of Nanoparticles Micro and Nano Technologies., V.-D. Hodoroaba, W. E. S. Unger, and A. G. Shard, eds. (Elsevier), pp. 349-371.

303. Cushman, C. V., Chatterjee, S., Major, G.H., Smith, N.J., Roberts, A., and Linford, M.R. (2016). Trends in Advanced XPS Instrumentation. 1. Overview of the Technique, Automation, High Sensitivity, Imaging, Snapshot Spectroscopy, Gas Cluster Ion Beams, and Multiple Analytical Techniques on the Instrument. Vac. Technol. Coat., 1-9.

304. Zhong, J.Q., Wang, M., Hoffmann, W.H., Van Spronsen, M.A., Lu, D., and Boscoboinik, J.A. (2018). Synchrotron-based ambient pressure X-ray photoelectron spectroscopy of hydrogen and helium. Appl. Phys. Lett. 112.

305. Castner, D.G., Hinds, K., and Grainger, D.W. (1996). X-ray Photoelectron Spectroscopy Sulfur $2 \mathrm{p}$ Study of Organic Thiol and Disulfide Binding Interactions with Gold Surfaces. Langmuir 12, 5083-5086.

306. Ghahremaninezhad, A., Dixon, D.G., and Asselin, E. (2013). Electrochemical and XPS analysis of chalcopyrite (CuFeS2) dissolution in sulfuric acid solution. Electrochim. Acta 87, 97-112.

307. Ko, T.H., and Chu, H. (2005). Spectroscopic study on sorption of hydrogen sulfide by means of red soil. Spectrochim. Acta - Part A Mol. Biomol. Spectrosc. 61, 2253-2259.

308. Vempati, S., Ertas, Y., Celebioglu, A., and Uyar, T. (2017). Tuning the degree of oxidation and electron delocalization of poly(3,4- 
ethylenedioxythiophene):poly(styrenesulfonate) with solid-electrolyte. Appl. Surf. Sci. 419, 770-777.

309. Spanninga, S.A., Martin, D.C., and Chen, Z. (2010). X-ray photoelectron spectroscopy study of counterion incorporation in poly(3,4ethylenedioxythiophene) (PEDOT) 2: Polyanion effect, toluenesulfonate, and small anions. J. Phys. Chem. C 114, 14992-14997.

310. Greczynski, G., Kugler, T., Keil, M., Osikowicz, W., Fahlman, M., and Salaneck, W.R. (2001). Photoelectron spectroscopy of thin films of PEDOTPSS conjugated polymer blend: a mini-review and some new results. J. Electron Spectros. Relat. Phenomena 121, 1-17.

311. Wang, C., Chen, F., Sun, K., Chen, R., Li, M., Zhou, X., Sun, Y., Chen, D., and Wang, G. (2018). Contributed Review: Instruments for measuring Seebeck coefficient of thin film thermoelectric materials: A mini-review. Rev. Sci. Instrum. 89.

312. Fu, Q., Xiong, Y., Zhang, W., and Xu, D. (2017). A setup for measuring the Seebeck coefficient and the electrical resistivity of bulk thermoelectric materials. Rev. Sci. Instrum. 88 .

313. Zhu, Q., Kim, H.S., and Ren, Z. (2017). A rapid method to extract Seebeck coefficient under a large temperature difference. Rev. Sci. Instrum. 88 .

314. Li, Y., Hu, X., Zhou, S., Yang, L., Yan, J., Sun, C., and Chen, P. (2014). A facile process to produce highly conductive poly $(3\{\} 4-$, ethylenedioxythiophene) films for ITO-free flexible OLED devices. J. Mater. Chem. C 2, 916-924.

315. Fan, Z., and Ouyang, J. (2019). Thermoelectric Properties of PEDOT:PSS. Adv. Electron. Mater. 5, 1-23.

316. Culebras, M., Choi, K., and Cho, C. (2018). Recent Progress in Flexible Organic Thermoelectrics. Micromachines 9, 638 .

317. Synoradzki, K., Ciesielski, K., Veremchuk, I., Borrmann, H., Skokowski, P., $\mathbf{2 5 0} \mid \mathrm{P}$ a g e 
Szymański, D., Grin, Y., and Kaczorowski, D. (2019). Thermal and electronic transport properties of the half-heusler phase ScNiSb. Materials (Basel). 12, 111.

318. Klich, W., and Ohtaki, M. (2021). Thermoelectric properties of Mo-doped bulk In2O3 and prediction of its maximum ZT. Ceram. Int. 47, 18116-18121.

319. Li, Z., Sun, H., Hsiao, C.L., Yao, Y., Xiao, Y., Shahi, M., Jin, Y., Cruce, A., Liu, X., Jiang, Y., et al. (2018). A Free-Standing High-Output Power Density Thermoelectric Device Based on Structure-Ordered PEDOT:PSS. Adv. Electron. Mater. 4, 1-8.

320. Nuramdhani, I., Jose, M., Samyn, P., Adriaensens, P., Malengier, B., Deferme, W., De Mey, G., and Van Langenhove, L. (2019). Charge-discharge characteristics of textile energy storage devices having different PEDOT:PSS ratios and conductive yarns configuration. Polymers (Basel). 11.

321. Horikawa, M., Fujiki, T., Shirosaki, T., Ryu, N., Sakurai, H., Nagaoka, S., and Ihara, H. (2015). On the mechanism of conductivity enhancement in poly(3,4ethylenedioxythiophene):poly(styrene sulfonate) film through solvent treatment. J. Mater. Chem. C 3, 8881-8887.

322. Petsagkourakis, I., Pavlopoulou, E., Portale, G., Kuropatwa, B. a, Dilhaire, S., Fleury, G., and Hadziioannou, G. (2016). Structurally-driven Enhancement of Thermoelectric Properties within Poly(3,4-ethylenedioxythiophene) thin Films. Sci. Rep. 6, 30501.

323. Wu, X., Liu, J., Wu, D., Zhao, Y., Shi, X., Wang, J., Huang, S., and He, G. (2014). Highly conductive and uniform graphene oxide modified PEDOT:PSS electrodes for ITO-Free organic light emitting diodes. J. Mater. Chem. C 2, 4044-4050.

324. Kyaw, A.K.K., Yemata, T.A., Wang, X., Lim, S.L., Chin, W.S., Hippalgaonkar, K., and Xu, J. (2018). Enhanced Thermoelectric Performance of PEDOT:PSS 
Films by Sequential Post-Treatment with Formamide. Macromol. Mater. Eng. $303,1-7$.

325. Wu, X., Lian, L., Yang, S., and He, G. (2016). Highly conductive PEDOT:PSS and graphene oxide hybrid film from a dipping treatment with hydroiodic acid for organic light emitting diodes. J. Mater. Chem. C 4, 8528-8534.

326. Pasha, A., Roy, A.S., Murugendrappa, M. V., Al-Hartomy, O.A., and Khasim, S. (2016). Conductivity and dielectric properties of PEDOT-PSS doped DMSO nano composite thin films. J. Mater. Sci. Mater. Electron. 27, 8332-8339.

327. Pasha, A., Khasim, S., Al-Hartomy, O.A., Lakshmi, M., and Manjunatha, K.G. (2018). Highly sensitive ethylene glycol-doped PEDOT-PSS organic thin films for LPG sensing. RSC Adv. 8, 18074-18083.

328. Poudel, B., Hao, Q., Ma, Y., Lan, Y., Minnich, A., Yu, B., Yan, X., Wang, D., Muto, A., Vashaee, D., et al. (2008). High-thermoelectric performance of nanostructured bismuth antimony telluride bulk alloys. Science (80-. ). 320, 634-638.

329. Jikei, M., Yamaya, T., Uramoto, S., and Matsumoto, K. (2014). Conductivity enhancement of PEDOT/PSS films by solvent vapor treatment. Int. J. Soc. Mater. Eng. Resour. 20, 158-162.

330. Haglund, R.F., Johnson, S.L., and Park, H.K. (2007). Electronic and optical properties of polymer thin films deposited by resonant infrared laser ablation. J. Laser Micro Nanoeng. 2, 234-240.

331. Liu, E., Liu, C., Zhu, Z., Shi, H., and Jiang, Q. (2015). Enhanced thermoelectric performance of PEDOT : PSS films by solvent thermal treatment. 1-5.

332. Ely, F., Matsumoto, A., Zoetebier, B., Peressinotto, V.S., Hirata, M.K., De Sousa, D.A., and Maciel, R. (2014). Handheld and automated ultrasonic spray deposition of conductive PEDOT:PSS films and their application in AC EL devices. Org. Electron. 15, 1062-1070.

333. Khodakarimi, S., Hekhmatshoar, M.H., Nasiri, M., Khaleghi Moghaddam, M., 
and Abbasi, F. (2016). Effects of process and post-process treatments on the electrical conductivity of the PEDOT:PSS films. J. Mater. Sci. Mater. Electron. 27, 1278-1285.

334. Bouhassoune, M., Mensfoort, S.L.M. va., Bobbert, P.A., and Coehoorn, R. (2009). Carrier-density and field-dependent charge-carrier mobility in organic semiconductors with correlated Gaussian disorder. Org. Electron. 10, 437-445.

335. Karl, N. (2003). Karl03Sm. Synth. Met. 134, 1-9.

336. Cowen, L.M., Atoyo, J., Carnie, M.J., Baran, D., and Schroeder, B.C. (2017). Review-Organic Materials for Thermoelectric Energy Generation. ECS J. Solid State Sci. Technol. 6, N3080-N3088.

337. Wei, Q., Mukaida, M., Kirihara, K., Naitoh, Y., and Ishida, T. (2015). Recent progress on PEDOT-based thermoelectric materials. Materials (Basel). 8, 732750.

338. Atoyo, J., Burton, M.R., Mcgettrick, J., and Carnie, M.J. (2020). Enhanced Electrical Conductivity and Seebeck Coefficient in PEDOT : PSS via a Two Step Ionic liquid and NaBH 4 Treatment for Organic Thermoelectrics. 12, 115 .

339. Cheng, Y.C., and Silbey, R.J. (2008). A unified theory for charge-carrier transport in organic crystals. J. Chem. Phys. 128.

340. Hokazono, M., Anno, H., and Toshima, N. (2014). Thermoelectric properties and thermal stability of PEDOT:PSS films on a polyimide substrate and application in flexible energy conversion devices. J. Electron. Mater. 43, 2196 2201.

341. Hinckley, A.C., Andrews, S.C., Dunham, M.T., Sood, A., Barako, M.T., Schneider, S., Toney, M.F., Goodson, K.E., and Bao, Z. (2021). Achieving High Thermoelectric Performance and Metallic Transport in Solvent-Sheared PEDOT:PSS. Adv. Electron. Mater. 7, 1-9. 
342. Girtan, M., Mallet, R., Caillou, D., Rusu, G.G., and Rusu, M. (2009). Thermal stability of poly(3,4-ethylenedioxythiophene)-polystyrenesulfonic acid films electrical properties. Superlattices Microstruct. 46, 44-51.

343. Vitoratos, E. (2012). Conductivity Degradation Study of PEDOT: PSS Films under Heat Treatment in Helium and Atmospheric Air. Open J. Org. Polym. Mater. 02, 7-11.

344. Cho, W., Im, S., Kim, S., Kim, S., and Kim, J.H. (2016). Synthesis and characterization of PEDOT: P(SS-co-VTMS) with hydrophobic properties and excellent thermal stability. Polymers (Basel). 8.

345. Skrebets, T.E., Paltser, R.S., and Ivahnov, A.D. (2018). The Thermal Stability of 1,4-Dioxane at Sub- and Supercritical Temperatures. Russ. J. Phys. Chem. B $12,1225-1228$.

346. Takano, T., Masunaga, H., Fujiwara, A., Okuzaki, H., and Sasaki, T. (2012). PEDOT nanocrystal in highly conductive PEDOT:PSS polymer films. Macromolecules 45, 3859-3865.

347. Zabihi, F., Xie, Y., Gao, S., and Eslamian, M. (2015). Morphology, conductivity, and wetting characteristics of PEDOT:PSS thin films deposited by spin and spray coating. Appl. Surf. Sci. 338, 163-177.

348. Modarresi, M., Franco-Gonzalez, J.F., and Zozoulenko, I. (2019). Computational microscopy study of the granular structure and $\mathrm{pH}$ dependence of PEDOT:PSS. Phys. Chem. Chem. Phys. 21, 6699-6711.

349. Li, J., Zhang, H., Liu, J., and Qi, H. (2012). Influence of alkaline treatment with ammonia water on the properties of PEDOT: PSS films. Int. J. Photoenergy 2012.

350. Fan, Z., Li, P., Du, D., and Ouyang, J. (2017). Significantly Enhanced Thermoelectric Properties of PEDOT:PSS Films through Sequential PostTreatments with Common Acids and Bases. Adv. Energy Mater. 7.

351. Kawahara, J., Ersman, P.A., Engquist, I., and Berggren, M. (2012). Improving 
the color switch contrast in PEDOT:PSS-based electrochromic displays. Org. Electron. 13, 469-474.

352. Mouhamad, Y., Mokarian-Tabari, P., Clarke, N., Jones, R.A.L., and Geoghegan, M. (2014). Dynamics of polymer film formation during spin coating. J. Appl. Phys. 116.

353. Ismail, N., Venault, A., Mikkola, J.P., Bouyer, D., Drioli, E., and Tavajohi Hassan Kiadeh, N. (2020). Investigating the potential of membranes formed by the vapor induced phase separation process. J. Memb. Sci. 597, 117601.

354. Li, Y., Hu, K., Han, X., Yang, Q., Xiong, Y., Bai, Y., Guo, X., Cui, Y., Yuan, C., Ge, H., et al. (2016). Phase Separation of Silicon-Containing Polymer/Polystyrene Blends in Spin-Coated Films. Langmuir 32, 3670-3678.

355. Guo, X., Liu, L., Zhuang, Z., Chen, X., Ni, M., Li, Y., Cui, Y., Zhan, P., Yuan, C., Ge, H., et al. (2015). A New Strategy of Lithography Based on Phase Separation of Polymer Blends. Sci. Rep. 5, 1-12.

356. Saghaei, J., Fallahzadeh, A., and Yousefi, M.H. (2015). Highly Conductive Poly(3,4-ethylenedioxythiophene):Poly(styrenesulfonate) Films Using 1Ethyl-3-methylimidazolium Tetracyanoborate Ionic Liquid. Org. Electron. physics, Mater. Appl. 19, 70-75.

357. Saxena, N., Pretzl, B., Lamprecht, X., Bießmann, L., Yang, D., Li, N., Bilko, C., Bernstorff, S., and Müller-Buschbaum, P. (2019). Ionic Liquids as PostTreatment Agents for Simultaneous Improvement of Seebeck Coefficient and Electrical Conductivity in PEDOT:PSS Films. ACS Appl. Mater. Interfaces 11, 8060-8071.

358. Bubnova, O., Khan, Z.U., Malti, A., Braun, S., Fahlman, M., Berggren, M., and Crispin, X. (2011). Optimization of the thermoelectric figure of merit in the conducting polymer poly(3,4-ethylenedioxythiophene). Nat. Mater. 10, 429433. 
359. Kee, S., Kim, H., Paleti, S.H.K., El Labban, A., Neophytou, M., Emwas, A.H., Alshareef, H.N., and Baran, D. (2019). Highly Stretchable and Air-Stable PEDOT:PSS/Ionic Liquid Composites for Efficient Organic Thermoelectrics. Chem. Mater. 31, 3519-3526.

360. Ma, S., Qiao, W., Cheng, T., Zhang, B., Yao, J., Alsaedi, A., Hayat, T., Ding, Y., Tan, Z., and Dai, S. (2018). Optical-Electrical-Chemical Engineering of PEDOT:PSS by Incorporation of Hydrophobic Nafion for Efficient and Stable Perovskite Solar Cells. ACS Appl. Mater. Interfaces 10, 3902-3911.

361. Hosseini, E., Ozhukil Kollath, V., and Karan, K. (2020). The key mechanism of conductivity in PEDOT:PSS thin films exposed by anomalous conduction behaviour upon solvent-doping and sulfuric acid post-treatment. J. Mater. Chem. C 8, 3982-3990.

362. Xing, W., Chen, Y., Wu, X., Xu, X., Ye, P., Zhu, T., Guo, Q., Yang, L., Li, W., and Huang, H. (2017). PEDOT:PSS-Assisted Exfoliation and Functionalization of 2D Nanosheets for High-Performance Organic Solar Cells. Adv. Funct. Mater. 27, 1-7.

363. Yemata, T.A., Zheng, Y., Kyaw, A.K.K., Wang, X., Song, J., Chin, W.S., and $\mathrm{Xu}, \mathrm{J}$. (2020). Modulation of the doping level of PEDOT:PSS film by treatment with hydrazine to improve the Seebeck coefficient. RSC Adv. 10, 1786-1792.

364. Atoyo, Jonathan, Matthew R Burton, Jmames Mcgettrick, M.J.C. Enhanced electrical conductivity and Seebeck coefficient in PEDOTPSS.

365. Lee, H.M., Anoop, G., Lee, H.J., Kim, W.S., and Jo, J.Y. (2019). Key parameters for enhancing the thermoelectric power factor of PEDOT:PSS/PANI-CSA multilayer thin films. RSC Adv. 9, 11595-11601.

366. Online, V.A., Culebras, M., and Cantarero, A. (2014). Enhanced thermoelectric performance of PEDOT with di ff erent counter-ions optimized by chemical. 10109-10115.

367. Yemata, T.A., Zheng, Y., Kyaw, A.K.K., Wang, X., Song, J., Chin, W.S., and $\mathrm{Xu}$, J. (2020). Improved Thermoelectric Properties and Environmental Stability 
of Conducting PEDOT:PSS Films Post-treated With Imidazolium Ionic Liquids. Front. Chem. 7, 1-16.

368. Zhu, Z., Liu, C., Jiang, Q., Shi, H., Jiang, F., Xu, J., Xiong, J., and Liu, E. (2015). Optimizing the thermoelectric properties of PEDOT:PSS films by combining organic co-solvents with inorganic base. J. Mater. Sci. Mater. Electron. 26, 8515-8521.

369. Badre, C., Marquant, L., Alsayed, A.M., and Hough, L.A. (2012). Highly Conductive Poly ( 3 , 4-ethylenedioxythiophene ): Poly ( styrenesulfonate ) Films Using 1-Ethyl-3-methylimidazolium Tetracyanoborate Ionic Liquid. $2723-2727$.

370. Sun, K., Zhang, S., Li, P., Xia, Y., Zhang, X., Du, D., Isikgor, F.H., and Ouyang, J. (2015). Review on application of PEDOTs and PEDOT:PSS in energy conversion and storage devices. J. Mater. Sci. Mater. Electron. 26, 4438-4462.

371. Kim, J., Kumar, R., Bandodkar, A.J., and Wang, J. (2017). Advanced Materials for Printed Wearable Electrochemical Devices: A Review. Adv. Electron. Mater. 3, 1-15.

372. Wang, X., Meng, F., Tang, H., Gao, Z., Li, S., Jiang, F., and Xu, J. (2017). An effective dual-solvent treatment for improving the thermoelectric property of PEDOT:PSS with white graphene. J. Mater. Sci. 52, 9806-9818.

373. Wang, J., Cai, K., and Shen, S. (2015). A facile chemical reduction approach for effectively tuning thermoelectric properties of PEDOT films. Org. Electron. $17,151-158$.

374. Beretta, D., Barker, A.J., Maqueira-Albo, I., Calloni, A., Bussetti, G., Dell'Erba, G., Luzio, A., Duò, L., Petrozza, A., Lanzani, G., et al. (2017). Thermoelectric Properties of Highly Conductive Poly(3,4ethylenedioxythiophene) Polystyrene Sulfonate Printed Thin Films. ACS Appl. Mater. Interfaces 9, 18151-18160. 
375. Fan, Z., Du, D., Yao, H., and Ouyang, J. (2017). Higher PEDOT Molecular Weight Giving Rise to Higher Thermoelectric Property of PEDOT:PSS: A Comparative Study of Clevios P and Clevios PH1000. ACS Appl. Mater. Interfaces 9, 11732-11738.

376. Tehrani, P., Hennerdal, L.-O., Dyer, A.L., Reynolds, J.R., and Berggren, M. (2009). Improving the contrast of all-printed electrochromic polymer on paper displays. J. Mater. Chem. 19, 1799-1802.

377. Levasseur, D., Mjejri, I., Rolland, T., and Rougier, A. (2019). Color tuning by oxide addition in PEDOT:PSS-based electrochromic devices. Polymers (Basel). 11, 1-12.

378. Fabretto, M., Hall, C., Vaithianathan, T., Innis, P.C., Mazurkiewicz, J., Wallace, G.G., and Murphy, P. (2008). The mechanism of conductivity enhancement in poly(3,4-ethylenedioxythiophene)-poly(styrenesulfonic) acid using linear-diol additives: Its effect on electrochromic performance. Thin Solid Films 516, $7828-7835$.

379. Zhang, X., Wu, J., Wang, J., Zhang, J., and Yang, Q. (2016). Solar Energy Materials \& Solar Cells Highly conductive PEDOT : PSS transparent electrode prepared by a post-spin-rinsing method for ef fi cient ITO-free polymer solar cells. Sol. Energy Mater. Sol. Cells 144, 143-149.

380. Photovoltaics, I.O. (2014). Unraveling the Enhanced Electrical Conductivity of PEDOT : PSS Thin Films for Unraveling the Enhanced Electrical Conductivity of PEDOT : PSS Thin Films for ITO-Free Organic Photovoltaics.

381. Saghaei, J., Fallahzadeh, A., and Yousefi, M.H. (2015). Highly Conductive Poly(3,4-ethylenedioxythiophene):Poly(styrenesulfonate) Films Using 1Ethyl-3-methylimidazolium Tetracyanoborate Ionic Liquid. Org. Electron. physics, Mater. Appl. 19, 70-75.

382. Teo, M.Y., Kim, N., Kee, S., Kim, B.S., Kim, G., Hong, S., Jung, S., and Lee, K. (2017). Highly stretchable and highly conductive PEDOT:PSS/Ionic liquid composite transparent electrodes for solution-processed stretchable electronics. 
ACS Appl. Mater. Interfaces 9, 819-826.

383. Li, Q., Yang, J., Chen, S., Zou, J., Xie, W., and Zeng, X. (2017). Highly Conductive PEDOT : PSS Transparent Hole Transporting Layer with Solvent Treatment for High Performance Silicon / Organic Hybrid Solar Cells.

384. Oh, H.J., Jang, J.G., Kim, J., Hong, J., Kim, J., Kwak, J., Kim, S.H., and Shin, S. (2017). Structural and Morphological Evolution for Water-resistant Organic Thermoelectrics. Sci. Rep., 1-8.

385. Spanninga, S.A. (2010). THE CHARACTERIZATION AND FABRICATION OF POLY ( 3 , 4-ETHYLENEDIOXYTHIOPHENE ) ENZYME BASED BIOSENSORS by.

386. Spanninga, S.A., Martin, D.C., and Chen, Z. (2010). Effect of anionic hydration on counterion incorporation in poly(3,4-ethylenedioxythiophene): An X-ray photoelectron spectroscopy study. J. Phys. Chem. C 114, 14998-15004.

387. Greczynski, G., Kugler, T., and Salaneck, W.R. (1999). Characterization of the PEDOT-PSS system by means of X-ray and ultraviolet photoelectron spectroscopy. 354, 129-135.

388. Mengistie, D.A., Ibrahem, M.A., Wang, P., and Chu, C. (2014). Highly conductive PEDOT : PSS treated with formic acid for ITO-free polymer solar cells Highly Conductive PEDOT : PSS Treated with Formic Acid for ITO-Free Polymer Solar Cells.

389. Khan, M.A., Armes, S.P., Perruchot, C., Ouamara, H., Chehimi, M.M., Greaves, S.J., and Watts, J.F. (2000). Surface Characterization of Poly ( 3 , 4ethylenedioxythiophene ) -Coated Latexes by X-ray Photoelectron Spectroscopy. 4171-4179.

390. Foelske-Schmitz, A., and Sauer, M. (2018). About charging and referencing of core level data obtained from X-ray photoelectron spectroscopy analysis of the ionic liquid/ultrahigh vacuum interface. J. Electron Spectros. Relat. Phenomena 
$224,51-58$.

391. Keppler, A., Himmerlich, M., Ikari, T., Marschewski, M., Maus-friedrichs, W., Endres, D.F., Pachomow, E., Ho, O., and Krischok, S. (2011). Changes of the near-surface chemical composition of the 1-ethyl-3-methylimidazolium bis ( trifluoromethylsulfonyl ) imide room temperature ionic liquid under the influence of irradiation. 1174-1181.

392. Wei, Q., Mukaida, M., Naitoh, Y., and Ishida, T. (2013). Morphological Change and Mobility Enhancement in PEDOT : PSS by Adding Co-solvents. 28312836.

393. Tsai, T.C., Chang, H.C., Chen, C.H., and Whang, W.T. (2011). Widely variable Seebeck coefficient and enhanced thermoelectric power of PEDOT:PSS films by blending thermal decomposable ammonium formate. Org. Electron. physics, Mater. Appl. 12, 2159-2164.

394. Massonnet, N., and Carella, A. (2014). Improvement of the Seebeck coefficient of PEDOT : PSS by chemical reduction combined with a novel method for its ... Improvement of the Seebeck coe ffi cient of PEDOT : PSS by chemical reduction combined with a novel method for its transfer using free-sta.

395. Wang, J., Cai, K., and Shen, S. (2015). High-Performance PEDOT:PSS Flexible Thermoelectric Materials and Their Devices by Triple Post-Treatments. Org. Electron. 17, 151-158.

396. Kok, M.M. De, Buechel, M., Vulto, S.I.E., Weijer, P. Van De, Meulenkamp, E.A., Winter, S.H.P.M. De, Mank, A.J.G., Vorstenbosch, H.J.M., and Weijtens, C.H.L. (2004). Modification of PEDOT : PSS as hole injection layer in polymer LEDs. 1359, 1342-1359.

397. Farah, A.A., Rutledge, S.A., Schaarschmidt, A., Lai, R., Freedman, J.P., and Helmy, A.S. (2012). Conductivity enhancement of poly(3,4ethylenedioxythiophene)- poly(styrenesulfonate) films post-spincasting. J. Appl. Phys. 112.

398. Nonoguchi, Y., Iihara, Y., Ohashi, K., Murayama, T., and Kawai, T. (2016). $\mathbf{2 6 0} \mid \mathrm{P}$ a g e 
Air-tolerant Fabrication and Enhanced Thermoelectric Performance of n-Type Single-walled Carbon Nanotubes Encapsulating 1,1'Bis(diphenylphosphino)ferrocene. Chem. - An Asian J. 11, 2423-2427.

399. Freeman, D.D., Choi, K., and Yu, C. (2012). N-Type Thermoelectric Performance of Functionalized Carbon Nanotube-Filled Polymer Composites. PLoS One 7.

400. Gerwig, R., Fuchsberger, K., Schroeppel, B., Link, G.S., Heusel, G., Kraushaar, U., Schuhmann, W., Stett, A., and Stelzle, M. (2012). PEDOT-CNT Composite Microelectrodes for Recording and Electrostimulation Applications: Fabrication, Morphology, and Electrical Properties. Front. Neuroeng. 5, 1-11.

401. Zhang, J., Gao, L., Sun, J., Liu, Y., Wang, Y., and Wang, J. (2012). Incorporation of single-walled carbon nanotubes with PEDOT/PSS in DMSO for the production of transparent conducting films. Diam. Relat. Mater. 22, 8287.

402. Bharti, M., Singh, A., Samanta, S., and Aswal, D.K. (2018). Conductive polymers for thermoelectric power generation. Prog. Mater. Sci. 93, 270-310.

403. Chong, X., Guan, P.W., Wang, Y., Shang, S.L., Paz Soldan Palma, J., Drymiotis, F., Ravi, V.A., Star, K.E., Fleurial, J.P., and Liu, Z.K. (2018). Understanding the Intrinsic P-Type Behavior and Phase Stability of Thermoelectric $\alpha-M g 3 S b 2$. ACS Appl. Energy Mater. 1, 6600-6608.

404. Cheng, X., Wang, X., and Chen, G. (2018). A convenient and highly tunable way to n-type carbon nanotube thermoelectric composite film using common alkylammonium cationic surfactant. J. Mater. Chem. A 6, 19030-19037.

405. Serrano-Claumarchirant, J.F., Culebras, M., Muñoz-Espí, R., Cantarero, A., Gómez, C.M., and Collins, M.N. (2020). PEDOT Thin Films with n-Type Thermopower. ACS Appl. Energy Mater. 3, 861-867.

406. Chaudhary, N., Singh, A., Aswal, D.K., Bharti, M., Sharma, A., Tillu, A.R., 261| P a g e 
Roy, M., Singh, B.P., Bahadur, J., Putta, V., et al. (2020). High energy electron beam induced improved thermoelectric properties of PEDOT:PSS films. Polymer (Guildf). 202, 122645.

407. Park, H., Lee, H., Kim, S., and Choi, H. (2014). Enhanced thermoelectric properties of PEDOT: PSS nano fi lms by a chemical dedoping process $\dagger$. 6532-6539.

408. Dargusch, M., Zou, J., and Chen, Z. (2019). High-Performance PEDOT:PSS Flexible Thermoelectric Materials and Their Devices by Triple PostTreatments.

409. Atnafu, T., Zheng, Y., Ko, A., Kyaw, K., Wang, X., Song, J., Shong, W., and $\mathrm{Xu}$, J. (2020). Sodium formaldehyde sulfoxylate, an ionic-type , water-soluble reducing reagent to effectively improve seebeck coefficient of PEDOT : PSS film. Org. Electron. 81, 105682.

410. Brownlie, L., and Shapter, J. (2018). Advances in carbon nanotube n-type doping: Methods, analysis and applications. Carbon N. Y. 126, 257-270.

411. Stavytska-barba, M., and Kelley, A.M. (2010). Surface Enhanced Raman Study of the Interaction of PEDOT : PSS with Silver and Gold Nanoparticles. Sol. Energy, 3-4.

412. Thu, M., Myint, Z., Nishi, T., Omoto, K., and Inoue, H. (2020). Controlling Electronic States of Few-walled Carbon Nanotube Yarn via Joule-annealing and p -type Doping Towards Large Thermoelectric Power Factor. 1-9.

413. Cowen, L.M., Atoyo, J., Carnie, M.J., Baran, D., and Schroeder, B.C. (2017). Review-Organic Materials for Thermoelectric Energy Generation. ECS J. Solid State Sci. Technol. 6, N3080-N3088.

414. Mazaheripour, A., Majumdar, S., Hanemann-rawlings, D., Thomas, E.M., Mcguiness, C., Alencon, L., Chabinyc, M.L., and Segalman, R.A. (2018). Tailoring the Seebeck Coefficient of PEDOT:PSS by Controlling Ion Stoichiometry in Ionic Liquid Additives. Chem. Mater. 30, 4816-4822. 
415. Liu, C., Xu, J., Lu, B., Yue, R., and Kong, F. (2012). Simultaneous increases in electrical conductivity and Seebeck coefficient of PEDOT:PSS films by adding ionic liquids into a polymer solution. J. Electron. Mater. 41, 639-645.

416. Li, Q., Deng, M., Zhang, S., Zhao, D., Jiang, Q., Guo, C., Zhou, Q., and Liu, W. (2019). Synergistic enhancement of thermoelectric and mechanical performances of ionic liquid LiTFSI modulated PEDOT flexible films. J. Mater. Chem. C 7, 4374-4381.

417. Ekholm, V., Vazdar, M., Mason, P.E., Bialik, E., Walz, M., Öhrwall, G., Rubensson, J., Jungwirth, P., Björneholm, O., Ekholm, V., et al. (2018). Anomalous surface behavior of hydrated guanidinium ions due to ion pairing Anomalous surface behavior of hydrated guanidinium ions due to ion pairing. 144508.

418. Goto, Y. (2017). Salt-induced formations of partially folded intermediates and amyloid fibrils suggests a common underlying mechanism.

419. Cheng, N., Li, W., Zhang, M., Wu, H., Sun, S., Zhao, Z., Xiao, Z., Sun, Z., Zi, W., and Fang, L. (2018). Enhance the performance and stability of methylammonium lead iodide perovskite solar cells with guanidinium thiocyanate additive. Curr. Appl. Phys.

420. Paper, F. (2019). E ff ects of guanidinium addition to $\mathrm{CH} 3 \mathrm{NH} 3 \mathrm{PbI} 3^{1}$. 491497.

421. Yang, Z., Cai, G., Bull, C.L., Tucker, M.G., Dove, M.T., Phillips, A.E., and Phillips, A.E. (2019). Hydrogen-bond-mediated structural variation of metal guanidinium formate hybrid perovskites under pressure Subject Areas : Author for correspondence :

422. Wo, M., and Kovalenko, M. V (2018). Guanidinium-Formamidinium Lead Iodide: A Layered Perovskite- Related Compound with Red Luminescence at Room Temperature. 10-13. 
423. Jodlowski, A.D., Roldán-carmona, C., Grancini, G., Salado, M., Ralaiarisoa, M., Ahmad, S., Koch, N., Camacho, L., and Miguel, G. De (2017). Large guanidinium cation mixed with methylammonium in lead iodide perovskites for $19 \%$ efficient solar cells. Nat. Energy 2.

424. Chavan, R.D., Prochowicz, D., Tavakoli, M.M., and Yadav, P. (2020). Surface Treatment of Perovskite Layer with Guanidinium Iodide Leads to Enhanced Moisture Stability and Improved Efficiency of Perovskite Solar Cells. 2000105, $1-9$.

425. Raj, C., Benoît, R., Dehez, F., and Chipot, C. (2018). Modeling induction phenomena in amino acid cation - interactions. Theor. Chem. Acc., 2-7.

426. Prakash, M., and Subramanian, T.V. V (2018). Guanidinium cation - water clusters. Theor. Chem. Acc.

427. Ashraf, M.A., Li, C., and Norouzi, F. (2020). Comptes Rendus Chimie.

428. Kim, J., Park, C., Im, S., Lee, H., and Kim, J.H. (2019). Effect of molecular weight distribution of PSSA on electrical conductivity of PEDOT:PSS. RSC Adv. 9, 4028-4034.

429. MacDiarmid, A.G., and Epstein, A.J. (1994). The concept of secondary doping as applied to polyaniline. Synth. Met. 65, 103-116.

430. Ouyang, J. (2013). "secondary doping" methods to significantly enhance the conductivity of PEDOT:PSS for its application as transparent electrode of optoelectronic devices. Displays 34, 423-436.

431. Horike, S., Misaki, M., Koshiba, Y., Saito, T., and Ishida, K. (2016). Enhanced thermoelectric power of single-wall carbon nanotube film blended with ionic liquid. Jpn. J. Appl. Phys. 55.

432. Nonoguchi, Y., Tani, A., Ikeda, T., Goto, C., Tanifuji, N., Uda, R.M., and Kawai, T. (2017). Water-Processable, Air-Stable Organic Nanoparticle-Carbon Nanotube Nanocomposites Exhibiting n-Type Thermoelectric Properties. Small $13,1-5$. 
433. Neves, J.C., de Castro, V.G., Assis, A.L.S., Veiga, A.G., Rocco, M.L.M., and Silva, G.G. (2018). In-situ determination of amine/epoxy and carboxylic/epoxy exothermic heat of reaction on surface of modified carbon nanotubes and structural verification of covalent bond formation. Appl. Surf. Sci. 436, 495504.

434. Feng-Xing, J., Jing-Kun, X., Bao-Yang, L., Yu, X., Rong-Jin, H., and Lai-Feng, L. (2008). Thermoelectric Performance of Poly(3,4-ethylenedioxythiophene): Poly(styrenesulfonate). Chinese Phys. Lett. 25, 2202.

435. Ihnatsenka, S., Crispin, X., and Zozoulenko, I. V (2015). Understanding hopping transport and thermoelectric properties of conducting polymers. $035201,1-12$.

436. Liu, H., Sung Choe, H., Chen, Y., Suh, J., Ko, C., Tongay, S., and Wu, J. (2017). Variable range hopping electric and thermoelectric transport in anisotropic black phosphorus. Appl. Phys. Lett. 111.

437. Petsagkourakis, I., Kim, N., Tybrandt, K., Zozoulenko, I., and Crispin, X. (2019). Poly(3,4-ethylenedioxythiophene): Chemical Synthesis, Transport Properties, and Thermoelectric Devices. Adv. Electron. Mater. 5, 1800918.

438. Ishida, A., Yamada, T., Nakano, T., Takano, Y., and Takaoka, S. (2011). Seebeck effects and electronic thermal conductivity of IV-VI materials. Jpn. J. Appl. Phys. 50, 1-24.

439. Hu, D., Liu, Q., Tisdale, J., Lei, T., Pei, J., Wang, H., Urbas, A., and Hu, B. Charge-transfer enhanced Seebeck effects in n-type and p-type organic polymers based on thin-film devices. 1-21.

440. Jaworski, C.M., Myers, R.C., Johnston-Halperin, E., and Heremans, J.P. (2012). Giant spin Seebeck effect in a non-magnetic material. Nature 487, 210-213.

441. Kumar, S., Munjal, S., and Khare, N. (2017). Metal-semiconductor transition and Seebeck inversion in CoFe2O4 nanoparticles. J. Phys. Chem. Solids 105, 
86-89.

442. Zhang, D., Wang, Y., and Yang, Y. (2019). Design , Performance, and Application of Thermoelectric Nanogenerators. 1805241, 1-13.

443. Stepien, L., Roch, A., Tkachov, R., Leupolt, B., Han, L., Ngo, N. Van, and Leyens, C. (2016). Thermal operating window for PEDOT : PSS fi lms and its related thermoelectric properties. Synth. Met.

444. Olaya, D., Hurtado-morales, M., Gómez, D., and Alejandro, O. (2017). Large thermoelectric figure of merit in graphene layered devices at low temperature.

445. Yusupov, K., Stumpf, S., You, S., Bogach, A., Martinez, P.M., Zakhidov, A., Schubert, U.S., Khovaylo, V., and Vomiero, A. (2018). Flexible Thermoelectric Polymer Composites Based on a Carbon Nanotubes Forest. 1801246, 1-11.

446. Worfolk, B.J., Andrews, S.C., Park, S., Reinspach, J., Liu, N., Toney, M.F., Mannsfeld, S.C.B., and Bao, Z. (2015). Ultrahigh electrical conductivity in solution-sheared polymeric transparent films. Proc. Natl. Acad. Sci. U. S. A. $112,14138-14143$.

447. Zhang, L., Harima, Y., and Imae, I. (2017). Highly improved thermoelectric performances of PEDOT : PSS / SWCNT composites by solvent treatment. Org. Electron. 51, 304-307.

448. Gharsallah, M., Nemes, N.M., Mompeán, F.J., and Mart, J.L. (2016). Giant Seebeck effect in Ge-doped SnSe. 1-9.

449. Zhao, D., Martinelli, A., Willfahrt, A., Fischer, T., Bernin, D., Khan, Z.U., Shahi, M., Brill, J., Jonsson, M.P., Fabiano, S., et al. for ultra-sensitive printed thermopiles. Nat. Commun., 1-8.

450. Luo, J., Cerretti, G., Krause, B., Zhang, L., Otto, T., Ullrich, M., Tremel, W., Voit, B., and Pötschke, P. (2016). Polypropylene-based melt mixed composites with singlewalled carbon nanotubes for thermoelectric applications: Switching from p-type to n-type by the addition of polyethylene glycol. Polymer (Guildf).

451. Shi, X., and Sun, J. (2017). Dependence of Seebeck Coefficient on the Density $\mathbf{2 6 6} \mid \mathrm{P}$ a g e 
of States in Organic Semiconductors. 3, 1-4.

452. Lee, W., Kang, Y.H., Lee, J.Y., Jang, K.S., and Cho, S.Y. (2016). Improving the thermoelectric power factor of CNT/PEDOT:PSS nanocomposite films by ethylene glycol treatment. RSC Adv. 6, 53339-53344.

453. Jeong, Y.S., and Akagi, K. (2011). Control of chirality and electrochromism in copolymer-type chiral PEDOT derivatives by means of electrochemical oxidation and reduction. Macromolecules 44, 2418-2426.

454. Series, C. (2019). Vacancies effect on graphene : Raman study Vacancies effect on graphene : Raman study.

455. Mehmood, T., Kim, J.H., Lee, D., Dizhur, S., Hirst, E.S., Iii, R.M.O., Sayyad, M.H., Munawar, M.A., and Xu, J. (2020). A flexible, printable , thin-film thermoelectric generator based on reduced graphene oxide - carbon nanotubes composites. J. Mater. Sci. 55, 10572-10581.

456. Aristizabal, K., Katzensteiner, A., Bachmaier, A., Mücklich, F., and Suarez, S. (2017). Study of the structural defects on carbon nanotubes in metal matrix composites processed by severe plastic deformation. Carbon N. Y. 125, 156161.

457. Tristant, D., Zubair, A., Puech, P., Neumayer, F., Moyano, S., Headrick, R.J., Tsentalovich, D.E., Young, C.C., Gerber, I.C., Pasquali, M., et al. (2016). Enlightening the ultrahigh electrical conductivities of doped double-wall carbon nanotube fi bers by Raman spectroscopy and fi rst-principles.

458. Zhong, H., Luo, Y., He, S., Tang, P., Li, D., Alonso-vante, N., and Feng, Y. (2016). Electrocatalytic cobalt nanoparticles interacting with nitrogen- doped carbon nanotube in-situ generated from a metal- organic framework for the Oxygen Reduction Reaction Electrocatalytic Cobalt Nanoparticles Interacting with Nitrogen-Doped Carbon Nanotub.

459. Wang, L., Zhang, J., Guo, Y., Chen, X., Jin, X., Yang, Q., Zhang, K., Wang, S., 267| P a g e 
and Qiu, Y. (2019). Fabrication of core-shell structured hybrids with enhanced thermoelectric power factors. Carbon N. Y. 148, 290-296.

460. Song, H., Qiu, Y., Wang, Y., Cai, K., Li, D., Deng, Y., and He, J. (2017). Polymer/carbon nanotube composite materials for flexible thermoelectric power generator. Compos. Sci. Technol.

461. Haziqah, N., Aziz, A., Jaafar, H., Sidek, R.M., Shafie, S., and Hamidon, M.N. (2019). Raman Study on Dispersion of Carbon Nanotube in Organic Solvent as The Preparation of Conductive. 2019 IEEE Reg. Symp. Micro Nanoelectron., $53-56$.

462. Li, C., Xu, S., Yue, Y., Yang, B., and Wang, X. (2016). Thermal characterization of carbon nanotube fi ber by time-domain differential Raman. Carbon N. Y. 103, 101-108.

463. Hohnholz, D., MacDiarmid, A.G., Sarno, D.M., and Jones, W.E. (2001). Uniform thin films of poly-3,4-ethylenedioxythiophene (PEDOT) prepared by in-situ deposition. Chem. Commun. 23, 2444-2445.

464. Ye, G., Xu, J., Ma, X., Zhou, Q., Li, D., Zuo, Y., Lv, L., Zhou, W., and Duan, X. (2017). One-step electrodeposition of free-standing flexible conducting PEDOT derivative film and its electrochemical capacitive and thermoelectric performance. Electrochim. Acta 224, 125-132.

465. Nitta, A., Kawahara, K., and Miyata, K. (2016). Characteristics Improvement of PEDOT:PSS Transparent Conductive Film Prepared by Ink-Jet Printing. Adv. Mater. Phys. Chem. 06, 239-247.

466. Ren, Y.X., Zhao, T.S., Liu, M., Zeng, Y.K., and Jiang, H.R. (2017). A selfcleaning Li-S battery enabled by a bifunctional redox mediator. J. Power Sources 361, 203-210.

467. Bichsel, Y., and Von Gunten, U. (1999). Determination of iodide and iodate by ion chromatography with postcolumn reaction and UV/visible detection. Anal. Chem. 71, 34-38. 
468. Massonnet, N., Carella, A., Jaudouin, O., and Rannou, P. (2014). Improvement of the Seebeck coe ffi cient of PEDOT : PSS by chemical reduction combined with a novel method for its transfer using free-standing. 1278-1283.

469. Wang, C., Sun, K., Fu, J., Chen, R., Li, M., Zang, Z., Liu, X., Li, B., Gong, H., and Ouyang, J. (2018). Enhancement of Conductivity and Thermoelectric Property of PEDOT:PSS via Acid Doping and Single Post-Treatment for Flexible Power Generator. Adv. Sustain. Syst. 2, 1800085.

470. Ju, D., Kim, D., Yook, H., Han, J.W., and Cho, K. (2019). Controlling Electrostatic Interaction in PEDOT:PSS to Overcome Thermoelectric Tradeoff Relation. Adv. Funct. Mater. 29, 1-9.

471. Wang, X., Ko, K., Yin, C., Wang, F., Zhu, Q., and Tang, T. (2018). Enhancement of thermoelectric performance of PEDOT : PSS fi lms by posttreatment with a superacid. RSC Adv. 8, 18334-18340.

472. Bredas, J.L., Themans, B., Andre, J.M., Chance, R.R., and Silbey, R. (1984). The role of mobile organic radicals and ions (solitons, polarons and bipolarons) in the transport properties of doped conjugated polymers. Synth. Met. 9, 265274.

473. Laokroekkiat, S., Hara, M., Nagano, S., and Nagao, Y. (2016). Metal - Organic Coordination Network Thin Film by Surface-Induced Assembly.

474. Kaloni, T.P., Giesbrecht, P.K., Schreckenbach, G., and Freund, M.S. (2017). Polythiophene : From Fundamental Perspectives to Applications.

475. Zozoulenko, I., Singh, A., Singh, S.K., Gueskine, V., Crispin, X., and Berggren, M. (2018). Polarons, Bipolarons, And Absorption Spectroscopy of PEDOT. ACS Appl. Polym. Mater. 1, 83-94.

476. Anna, K., and Moos, R. (2012). Why Does the Electrical Conductivity in PEDOT: PSS Decrease with PSS Content? A Study Combining Thermoelectric Measurements with Impedance Spectroscopy. 976-983. 
477. Flagg, L.Q., Giridharagopal, R., Guo, J., and Ginger, D.S. (2018). AnionDependent Doping and Charge Transport in Organic Electrochemical Transistors Anion-Dependent Doping and Charge Transport in Organic Electrochemical Transistors.

478. Romero, M., Mombrú, D., Pignanelli, F., and Faccio, R. (2020). Mini-Review : Mixed Ionic - Electronic Charge Carrier Localization and Transport in Hybrid Organic - Inorganic Nanomaterials. 8, 1-11.

479. Wieland, M., Dingler, C., Merkle, R., Maier, J., and Ludwigs, S. (2020). Humidity-Controlled Water Uptake and Conductivities in Ion and Electron Mixed Conducting Polythiophene Films.

480. Forsyth, M. Mixed Ionic-Electronic Conductors Based on PEDOT: PolyDADMA and Organic Ionic Plastic Crystals. 1-18.

481. Choi, K., Kim, S.L., Yi, S., Hsu, J., and Yu, C. (2018). Energy , Environmental , and Catalysis Applications Promoting Dual Electronic and Ionic Transport in PEDOT by Embedding Carbon Nanotubes for Large Thermoelectric Responses Promoting Dual Electronic and Ionic Transport in PEDOT by Embedding Carbon Nanotube.

482. Kayser, L. V, and Lipomi, D.J. (2018). Stretchable Conductive Polymers and Composites Based on PEDOT and PEDOT : PSS. 1806133, 1-13.

483. Lock, J.P., Im, S.G., and Gleason, K.K. (2006). Oxidative chemical vapor deposition of electrically conducting poly(3,4-ethylenedioxythiophene) films. Macromolecules 39, 5326-5329.

484. Li, S., Lin, Y., Xie, H., Zhang, S., and Xu, J. (2006). Brønsted Guanidine Acid - Base Ionic Liquids : Novel Reaction Media for the Palladium-Catalyzed Heck Reaction. 2003-2006.

485. Cui, X.Y., Tan, C.H., and Leow, D. (2019). Metal-catalysed reactions enabled by guanidine-type ligands. Org. Biomol. Chem. 17, 4689-4699.

486. Cho, B., and Wong, M.W. (2015). Unconventional bifunctional Lewis-Brønsted 
acid activation mode in bicyclic guanidine-catalyzed conjugate addition reactions. Molecules 20, 15108-15121.

487. Refat, M.S., El-Didamony, A.M., and Grabchev, I. (2007). UV-vis, IR spectra and thermal studies of charge transfer complex formed between poly(amidoamine) dendrimers and iodine. Spectrochim. Acta - Part A Mol. Biomol. Spectrosc. 67, 58-65.

488. Gogoi, P., Mohan, U., Borpuzari, M.P., Boruah, A., and Baruah, S.K. (2017). $\mathrm{UV}-\mathrm{V}$ is spectroscopy and density functional study of solvent effect on the charge transfer band of the $n \rightarrow \sigma^{*}$ complexes of 2-Methylpyridine and 2Chloropyridine with molecular iodine. J. Mol. Struct. 1131, 114-123.

489. Chan, K.H.K., Yamao, T., Kotaki, M., and Hotta, S. (2010). Unique structural features and electrical properties of electrospun conjugated polymer poly(3hexylthiophene) (P3HT) fibers. Synth. Met. 160, 2587-2595.

490. Park, S.H., Shin, K.H., Kim, J.Y., Yoo, S.J., Lee, K.J., Shin, J., Choi, J.W., Jang, J., and Sung, Y.E. (2012). The application of camphorsulfonic acid doped polyaniline films prepared on TCO-free glass for counter electrode of bifacial dye-sensitized solar cells. J. Photochem. Photobiol. A Chem. 245, 1-8.

491. Zhang, J., Xing, A., Jia, B., and Liu, X. (2019). Synthesis of conductive polyaniline nanofibers in one step by protonic acid and iodine doping. High Perform. Polym. 31, 893-900.

492. Chen, T.W., Tsai, T.H., Chen, S.M., and Lin, K.C. (2011). Using PEDOT film modified electrode to monitor iodide and its enhancement of arsenite sensing. Int. J. Electrochem. Sci. 6, 2043-2057.

493. Li, N., Shi, L., Wang, X., Guo, F., and Yan, C. (2011). Experimental Study of Closed System in the Chlorine Dioxide-Iodide-Sulfuric Acid Reaction by UVVis Spectrophotometric Method. Int. J. Anal. Chem. 2011, 1-7.

494. Abbasi, A.R., and Morsali, A. (2010). Formation of silver iodide nanoparticles $271 \mid \mathrm{P}$ a g e 
on silk fiber by means of ultrasonic irradiation. Ultrason. Sonochem. 17, 704710.

495. Wang, Y., Liu, X., Zhang, T., Wang, X., Kan, M., Shi, J., and Zhao, Y. (2019). The Role of Dimethylammonium Iodide in CsPbI3 Perovskite Fabrication: Additive or Dopant? Angew. Chemie - Int. Ed. 58, 16691-16696.

496. Kireev, S. V., and Shnyrev, S.L. (2015). Study of molecular iodine, iodate ions, iodide ions, and triiodide ions solutions absorption in the UV and visible light spectral bands. Laser Phys. 25, 75602.

497. Nasybulin, E., Wei, S., Cox, M., Kymissis, I., and Levon, K. (2011). Erratum: Morphological and spectroscopic studies of electrochemically deposited poly(3,4-ethylenedioxythiophene) (PEDOT) hole extraction layer for organic photovoltaic device (OPVd) fabrication". J. Phys. Chem. C 115, 17623.

498. Bönnemann, H., Khelashvili, G., Behrens, S., Hinsch, A., Skupien, K., and Dinjus, E. (2007). Role of the platinum nanoclusters in the iodide/triiodide redox system of dye solar cells. J. Clust. Sci. 18, 141-155.

499. Jono, R., Sumita, M., Tateyama, Y., and Yamashita, K. (2012). Redox reaction mechanisms with non-triiodide mediators in dye-sensitized solar cells by redox potential calculations. J. Phys. Chem. Lett. 3, 3581-3584.

500. Abe, R., Shinmei, K., Koumura, N., Hara, K., and Ohtani, B. (2013). Visiblelight-induced water splitting based on two-step photoexcitation between dyesensitized layered niobate and tungsten oxide photocatalysts in the presence of a triiodide/iodide shuttle redox mediator. J. Am. Chem. Soc. 135, 16872-16884.

501. Boschloo, G., and Hagfeldt, A. (2009). Characteristics of the iodide/triiodide redox mediator in dye-sensitized solar cells. Acc. Chem. Res. 42, 1819-1826.

502. Zhao, Y., Wang, L., and Byon, H.R. (2013). High-performance rechargeable lithium-iodine batteries using triiodide/iodide redox couples in an aqueous cathode. Nat. Commun. 4, 1896-1897.

503. Xiong, J., Jiang, F., Shi, H., Xu, J., Liu, C., Zhou, W., Jiang, Q., Zhu, Z., and 
$\mathrm{Hu}$, Y. (2015). Liquid exfoliated graphene as dopant for improving the thermoelectric power factor of conductive PEDOT:PSS nanofilm with hydrazine treatment. ACS Appl. Mater. Interfaces 7, 14917-14925.

504. Jiang, F., Xiong, J., Zhou, W., Liu, C., Wang, L., Zhao, F., Liu, H., and Xu, J. (2016). Use of organic solvent-assisted exfoliated MoS2 for optimizing the thermoelectric performance of flexible PEDOT:PSS thin films. J. Mater. Chem. A $4,5265-5273$.

505. Yan, H., and Okuzaki, H. (2009). Effect of solvent on PEDOT / PSS nanometerscaled thin films : XPS and STEM / AFM studies. 159, 2225-2228.

506. Li, K., Song, X., Wang, C., Mei, Y., Sun, X., and Ning, P. (2017). Deactivation mechanism of the simultaneous removal of carbonyl sulphide and carbon disulphide over $\mathrm{Fe}-\mathrm{Cu}-\mathrm{Ni} / \mathrm{MCSAC}$ catalysts. J. Chem. Sci. 129, 1893-1903.

507. Feng, Q., Wen, S., Deng, J., and Zhao, W. (2017). Combined DFT and XPS investigation of enhanced adsorption of sulfide species onto cerussite by surface modification with chloride. Appl. Surf. Sci. 425, 8-15.

508. Tabakovic, I., Riemer, S., Tabakovic, K., Sun, M., and Kief, M. (2006). Mechanism of Saccharin Transformation to Metal Sulfides and Effect of Inclusions on Corrosion Susceptibility of Electroplated CoFe Magnetic Films. J. Electrochem. Soc. 153, C586.

509. Krylova, V., and Andruleviçius, M. (2009). Optical, XPS and XRD studies of semiconducting copper sulfide layers on a polyamide film. Int. J. Photoenergy 2009.

510. Vesel, A., Kovac, J., Primc, G., Junkar, I., and Mozetic, M. (2016). Effect of $\mathrm{H} 2 \mathrm{~S}$ plasma treatment on the surface modification of a polyethylene terephthalate surface. Materials (Basel). 9.

511. Jiang, Q., Liu, C., Song, H., Shi, H., Yao, Y., Xu, J., Zhang, G., and Lu, B. (2013). Improved thermoelectric performance of PEDOT:PSS films prepared 
by polar-solvent vapor annealing method. J. Mater. Sci. Mater. Electron. 24, $4240-4246$.

512. Kara, M.O.P., and Frey, M.W. (2014). Effects of Solvents on the Morphology and Conductivity of Poly ( 3 , 4-ethylenedioxythiophene ): Poly ( styrenesulfonate ) Nanofibers. 40305, 1-8.

513. Li, K., Zhao, Y., Zhang, P., He, C., Deng, J., Ding, S., and Shi, W. (2016). Combined DFT and XPS investigation of iodine anions adsorption on the sulfur terminated (001) chalcopyrite surface. Appl. Surf. Sci.

514. Zhang, Q., Wu, Z., Liu, F., Liu, S., Liu, J., Wang, Y., and Yan, T. (2017). Encapsulating a high content of iodine into an active graphene substrate as a cathode material for. J. Mater. Chem. A Mater. energy Sustain. 5, 15235-15242.

515. Ameen, S., Song, M., Kim, D.G., Im, Y. Bin, Seo, H.K., Kim, Y.S., and Shin, H.S. (2012). Iodine doped polyaniline thin film for heterostructure devices via PECVD technique: Morphological, structural, and electrical properties. Macromol. Res. 20, 30-36.

516. Wang, F., Liu, Z., Yang, C., Zhong, H., Nam, G., Zhang, P., Dong, R., Wu, Y., Cho, J., Zhang, J., et al. (2020). Fully Conjugated Phthalocyanine Copper Metal-Organic Frameworks for Sodium-Iodine Batteries with Long-TimeCycling Durability. Adv. Mater. 32.

517. Song, S., Wang, C., Hong, F., He, Z., Cai, Q., and Chen, J. (2011). Galliumand iodine-co-doped titanium dioxide for photocatalytic degradation of 2chlorophenol in aqueous solution: Role of gallium. Appl. Surf. Sci. 257, 3427 3432.

518. Rocks, C., Svrcek, V., Maguire, P., and Mariotti, D. (2017). Understanding surface chemistry during MAPbI3 spray deposition and its effect on photovoltaic performance. J. Mater. Chem. C 5, 902-916.

519. Han, Q., Hu, C., Zhao, F., Zhang, Z., Chen, N., and Qu, L. (2015). One-step preparation of iodine-doped graphitic carbon nitride nanosheets as efficient photocatalysts for visible light water splitting. J. Mater. Chem. A 3, 4612-4619. 
520. Brezová, V., Jankovičová, M., Soldán, M., Blažková, A., Reháková, M., Šurina, I., Čeppan, M., and Havlínová, B. (1994). Photocatalytic degradation of ptoluenesulphonic acid in aqueous systems containing powdered and immobilized titanium dioxide. J. Photochem. Photobiol. A Chem. 83, 69-75.

521. Van Ijzendoorn, L.J., De Jong, M.P., Janssen, F.J.J., Andersson, G.G., Sturm, J.M., and De Voigt, M.J.A. (2002). Ion beam analysis at cryogenic temperatures for polymer light emitting diodes. Nucl. Instruments Methods Phys. Res. Sect. B Beam Interact. with Mater. Atoms 188, 36-40.

522. Osikowicz, W., Van Der Gon, A.W.D., Crispin, X., De Jong, M.P., Friedlein, R., Groenendaal, L., Fahlman, M., Beljonne, D., Lazzaroni, R., and Salaneck, W.R. (2003). A joint theoretical and experimental study on the electronic properties of phenyl-capped 3,4-ethylenedioxythiophene oligomers. J. Chem. Phys. 119, 10415-10420.

523. Uchida, K., Saito, M., Murakami, A., Nakamura, S., and Irie, M. (2003). Nondestructive readout of the photochromic reactions of diarylethene derivatives using infrared light. Adv. Mater. 15, 121-125.

524. Mengistie, D.A., Ibrahem, M.A., Wang, P.C., and Chu, C.W. (2014). Highly conductive PEDOT:PSS treated with formic acid for ITO-free polymer solar cells. ACS Appl. Mater. Interfaces 6, 2292-2299.

525. Robert, A. (2020). PEDOT Electrodes for Improving Multiple Facets of Neurochemical Measurements.

526. Liu, M., Meng, Q., Yang, Z., Zhao, X., and Liu, T. (2018). Ultra-long-term cycling stability of an integrated carbon-sulfur membrane with dual shuttleinhibiting layers of graphene "nets" and a porous carbon skin. Chem. Commun. $54,5090-5093$.

527. Meng, L., Turner, A.P.F., and Mak, W.C. (2019). Modulating Electrode Kinetics for Discrimination of Dopamine by a PEDOT:COOH Interface Doped 
with Negatively Charged Tricarboxylate. ACS Appl. Mater. Interfaces 11, 34497-34506.

528. Shi, Q., Qin, B., Liang, H., Gao, Y., Bi, Q., and Qu, B. (2018). Effects of igneous intrusions on the structure and spontaneous combustion propensity of coal: A case study of bituminous coal in Daxing Mine, China. Fuel 216, 181189.

529. Ateia, M., Ceccato, M., Budi, A., Ataman, E., Yoshimura, C., and Johnson, M.S. (2018). Ozone-assisted regeneration of magnetic carbon nanotubes for removing organic water pollutants. Chem. Eng. J. 335, 384-391.

530. Ivanko, I., Svoboda, J., Lukešová, M., Šeděnková, I., and Tomšík, E. (2020). Hydrogen Bonding as a Tool to Control Chain Structure of PEDOT: Electrochemical Synthesis in the Presence of Different Electrolytes. Macromolecules 53, 2464-2473.

531. Chen, X., Wang, X., and Fang, D. (2020). A review on C1s XPS-spectra for some kinds of carbon materials. Fullerenes Nanotub. Carbon Nanostructures 0 , $1-11$.

532. Theodosiou, A., Spencer, B.F., Counsell, J., and Jones, A.N. (2020). An XPS/UPS study of the surface/near-surface bonding in nuclear grade graphites: A comparison of monatomic and cluster depth-profiling techniques. Appl. Surf. Sci. $508,144764$.

533. Guo, Z., Zhang, J., Kang, Y., and Liu, H. (2017). Rapid and efficient removal of $\mathrm{Pb}$ (II) from aqueous solutions using biomass-derived activated carbon with humic acid in-situ modification. Ecotoxicol. Environ. Safetyfile///C/Users/kaizo/Documents/printed TEGS/araujo2016.pdf 145, 442448.

534. Hu, M., Luber, E.J., and Buriak, J.M. (2020). Reconsidering X-ray Photoelectron Spectroscopy Quantification of Substitution Levels of Monolayers on Unoxidized Silicon Surfaces. J. Phys. Chem. C 124, 1646116477. 
535. Takeuchi, K., Yamamoto, S., Hamamoto, Y., Shiozawa, Y., Tashima, K., Fukidome, H., Koitaya, T., Mukai, K., Yoshimoto, S., Suemitsu, M., et al. (2017). Adsorption of CO2 on Graphene: A Combined TPD, XPS, and vdWDF Study. J. Phys. Chem. C 121, 2807-2814.

536. Bañuls-Ciscar, J., Pratelli, D., Abel, M.L., and Watts, J.F. (2016). Surface characterisation of pine wood by XPS. Surf. Interface Anal. 48, 589-592.

537. Canepa, P., Gonella, G., Pinto, G., Grachev, V., Canepa, M., and Cavalleri, O. (2019). Anchoring of Aminophosphonates on Titanium Oxide for Biomolecular Coupling. J. Phys. Chem. C 123, 16843-16850.

538. Rosly, N.Z., Ahmad, S.A.A., Abdullah, J., and Yusof, N.A. (2016). Patterned array of poly(Ethylene glycol) silane monolayer for label-free detection of dengue. Sensors (Switzerland) 16,1-11.

539. Bhatt, S., Pulpytel, J., Mirshahi, M., and Arefi-Khonsari, F. (2012). Catalystfree plasma-assisted copolymerization of poly( $\varepsilon$-caprolactone) -poly(ethylene glycol) for biomedical applications. ACS Macro Lett. 1, 764-767.

540. Zoulalian, V., Zürcher, S., Tosatti, S., Textor, M., Monge, S., and Robin, J.J. (2010). Self-Assembly of poly (ethylene glycol)-poly (alkyl phosphonate) terpolymers on titanium oxide surfaces: synthesis, interface characterization, investigation of nonfouling properties, and long-term stability. Langmuir 26, $74-82$.

541. Wang, Z., Long, P., Feng, Y., Qin, C., and Feng, W. (2017). Surface passivation of carbon dots with ethylene glycol and their high-sensitivity to Fe3+. RSC Adv. 7, 2810-2816.

542. Keyvani, M., Afrand, M., Toghraie, D., and Reiszadeh, M. (2018). An experimental study on the thermal conductivity of cerium oxide/ethylene glycol nanofluid: developing a new correlation. J. Mol. Liq. 266, 211-217.

543. H.M., T., and W., P. (1935). Glycol-Water Mixtures. Ind. Eng. Chem. 27, 66277| P a g e 
68.

544. Rusdi, M., Moroi, Y., Nakahara, H., and Shibata, O. (2005). Evaporation from water-ethylene glycol liquid mixture. Langmuir 21, 7308-7310.

545. Clarke, C.J., Maxwell-Hogg, S., Smith, E.F., Hawker, R.R., Harper, J.B., and Licence, P. (2019). Resolving X-ray photoelectron spectra of ionic liquids with difference spectroscopy. Phys. Chem. Chem. Phys. 21, 114-123.

546. Santos, A.R., Blundell, R.K., and Licence, P. (2015). XPS of guanidinium ionic liquids: A comparison of charge distribution in nitrogenous cations. Phys. Chem. Chem. Phys. 17, 11839-11847.

547. Ling, X., Yuan, J., Zhang, X., Qian, Y., Zakeeruddin, S.M., Larson, B.W., Zhao, Q., Shi, J., Yang, J., Ji, K., et al. (2020). Guanidinium-Assisted Surface Matrix Engineering for Highly Efficient Perovskite Quantum Dot Photovoltaics. Adv. Mater. 32, 1-9.

548. Hong Anh Ngo, T., Mori, S., and Thi Tran, D. (2017). Photo-induced grafting of poly(ethylene glycol) onto polyamide thin film composite membranes. J. Appl. Polym. Sci. 134, 1-9.

549. Chen, Y., and Luo, S.C. (2019). Synergistic Effects of Ions and Surface Potentials on Antifouling Poly(3,4-ethylenedioxythiophene): Comparison of Oligo(Ethylene Glycol) and Phosphorylcholine. Langmuir 35, 1199-1210.

550. Antol, I., Glasovac, Z., Crespo-Otero, R., and Barbatti, M. (2014). Guanidine and guanidinium cation in the excited state - Theoretical investigation. J. Chem. Phys. 141 .

551. Khalaf, M.S., Oakley, S.H., Coles, M.P., and Hitchcock, P.B. (2008). A strategy for the propagation of hydrogen-bonding in bicyclic guanidinium salts. CrystEngComm 10, 1653-1661.

552. Xu, B., Jacobs, M.I., Kostko, O., and Ahmed, M. (2017). Guanidinium Group Remains Protonated in a Strongly Basic Arginine Solution. ChemPhysChem $18,1503-1506$. 
553. Raczyńska, E.D., Cyrański, M.K., Gutowski, M., Rak, J., Gal, J.F., Maria, P.C., Darowska, M., and Duczmal, K. (2003). Consequences of proton transfer in guanidine. J. Phys. Org. Chem. 16, 91-106.

554. Tan, C.H., and Coles, M. (2014). The chemistry of guanidine, guanidinium, and guanidinate compounds. Aust. J. Chem. 67, 963-964.

555. Werner, J., Wernersson, E., Ekholm, V., Ottosson, N., Öhrwall, G., Heyda, J., Persson, I., Söderström, J., Jungwirth, P., and Björneholm, O. (2014). Surface behavior of hydrated guanidinium and ammonium ions: A comparative study by photoelectron spectroscopy and molecular dynamics. J. Phys. Chem. B 118, $7119-7127$.

556. Hsu, J.C., Lin, Y.H., and Wang, P.W. (2020). X-ray photoelectron spectroscopy analysis of nitrogen-doped $\mathrm{TiO} 2$ films prepared by reactive-ion-beam sputtering with various $\mathrm{NH} 3 / \mathrm{O} 2$ gas mixture ratios. Coatings 10 .

557. Santos, A.R., Blundell, R.K., and Licence, P. (2015). XPS of guanidinium ionic liquids: A comparison of charge distribution in nitrogenous cations. Phys. Chem. Chem. Phys. 17, 11839-11847.

558. Ma, Q., Guo, H., Gordon, R.G., and Zaera, F. (2011). Surface chemistry of copper(I) acetamidinates in connection with atomic layer deposition (ALD) processes. Chem. Mater. 23, 3325-3334.

559. Dougherty, D.A. (2013). The cation- $\pi$ interaction. Acc. Chem. Res. 46, 885893.

560. Frontera, A., Quiñonero, D., and Deyà, P.M. (2011). Cation- $\pi$ and anion- $\pi$ interactions. Wiley Interdiscip. Rev. Comput. Mol. Sci. 1, 440-459.

561. Ryzhov, V., Dunbar, R.C., Cerda, B., and Wesdemiotis, C. (2000). Cation- $\pi$ effects in the complexation of $\mathrm{Na}+$ and $\mathrm{K}+$ with $\mathrm{Phe}$, Tyr, and Trp in the gas phase. J. Am. Soc. Mass Spectrom. 11, 1037-1046. 
562. Kumar, K., Woo, S.M., Siu, T., Cortopassi, W.A., Duarte, F., and Paton, R.S. (2018). Cation- $\pi$ interactions in protein-ligand binding: Theory and data-mining reveal different roles for lysine and arginine. Chem. Sci. 9, 2655-2665.

563. Trujillo, C., Rodriguez-Sanz, A.A., and Rozas, I. (2015). Aromatic amino acidsguanidinium complexes through cation- $\varphi$ Interactions. Molecules 20, 9214 9228.

564. Weathers, A., Khan, Z.U., Brooke, R., Evans, D., Pettes, M.T., Andreasen, J.W., Crispin, X., and Shi, L. (2015). Significant electronic thermal transport in the conducting polymer poly(3,4-ethylenedioxythiophene). Adv. Mater. 27, 2101-2106.

565. Horii, T., Hikawa, H., Katsunuma, M., and Okuzaki, H. (2018). Synthesis of highly conductive PEDOT:PSS and correlation with hierarchical structure. Polymer (Guildf). 140, 33-38.

566. Gordon, M.P., Zaia, E.W., Zhou, P., Russ, B., Coates, N.E., Sahu, A., and Urban, J.J. (2017). Soft PEDOT:PSS aerogel architectures for thermoelectric applications. J. Appl. Polym. Sci. 134, 1-9.

567. Savva, A., Wustoni, S., and Inal, S. (2018). Ionic-to-electronic coupling efficiency in PEDOT:PSS films operated in aqueous electrolytes. J. Mater. Chem. C 6, 12023-12030.

568. Wu, F., Li, P., Sun, K., Zhou, Y., Chen, W., Fu, J., Li, M., Lu, S., Wei, D., Tang, X., et al. (2017). Conductivity Enhancement of PEDOT:PSS via Addition of Chloroplatinic Acid and Its Mechanism. Adv. Electron. Mater. 3, 1-10.

569. Bießmann, L., Saxena, N., Hohn, N., Hossain, M.A., Veinot, J.G.C., and Müller-Buschbaum, P. (2019). Highly Conducting, Transparent PEDOT:PSS Polymer Electrodes from Post-Treatment with Weak and Strong Acids. Adv. Electron. Mater. 5, 1-10.

570. Yemata, T.A., Kyaw, A.K.K., Zheng, Y., Wang, X., Zhu, Q., Chin, W.S., and $\mathrm{Xu}$, J. (2020). Enhanced thermoelectric performance of poly(3,4ethylenedioxythiophene):poly(4-styrenesulfonate) (PEDOT:PSS) with long- 
term humidity stability via sequential treatment with trifluoroacetic acid. Polym. Int. 69, 84-92.

571. Saxena, N., Pretzl, B., Lamprecht, X., Bießmann, L., Yang, D., Li, N., Bilko, C., Bernstor, S., and Mu, P. (2019). Ionic Liquids as Post-Treatment Agents for Simultaneous Improvement of Seebeck Coe ffi cient and Electrical Conductivity in PEDOT : PSS Films.

572. Gharahcheshmeh, M.H., Tavakoli, M.M., Gleason, E.F., Robinson, M.T., Kong, J., and Gleason, K.K. (2019). Tuning, optimization, and perovskite solar cell device integration of ultrathin poly(3,4-ethylene dioxythiophene) films via a single-step all-dry process. Sci. Adv. 5, 1-13.

573. Fan, J., Rezaie, S.S., Facchini-Rakovich, M., Gudi, D., Montemagno, C., and Gupta, M. (2019). Tuning PEDOT:PSS conductivity to obtain complementary organic electrochemical transistor. Org. Electron. 66, 148-155.

574. Sarabia-Riquelme, R., Andrews, R., Anthony, J.E., and Weisenberger, M.C. (2020). Highly conductive wet-spun PEDOT:PSS fibers for applications in electronic textiles. J. Mater. Chem. C.

575. Pistillo, B.R., Menguelti, K., Desbenoit, N., Arl, D., Leturcq, R., Ishchenko, O.M., Kunat, M., Baumann, P.K., and Lenoble, D. (2016). One step deposition of PEDOT films by plasma radicals assisted polymerization: Via chemical vapour deposition. J. Mater. Chem. C 4, 5617-5625.

576. Sugawara, K., Kato, T., Nagai, Y., Kitajima, Y., and Science, R. (2016). Desulphurization of Coal Soluble Using Ionic Liquids Faculty of Engineering and Resource Science, Akita University ,. 33, 2015-2017.

577. Meek, K.M., and Elabd, Y.A. (2016). Sulfonated Polymerized Ionic Liquid Block Copolymers. Macromol. Rapid Commun. 37, 1200-1206.

578. Królikowski, M., and Lipińska, A. (2019). Separation of thiophene, or benzothiophene from model fuel using glycols. Liquid-liquid phase equilibria 
and oxidative desulfurization study. Fluid Phase Equilib. 482, 11-23.

579. Patterson, D.A., Metcalfe, I.S., Xiong, F., and Livingston, A.G. (2002). Biodegradability of linear alkylbenzene sulfonates subjected to wet air oxidation. J. Chem. Technol. Biotechnol. 77, 1039-1049.

580. Gao, H., Zeng, S., Liu, X., Nie, Y., Zhang, X., and Zhang, S. (2015). Extractive desulfurization of fuel using N-butylpyridinium-based ionic liquids. RSC Adv. 5, 30234-30238.

581. Wang, L., Jin, G., and Xu, Y. (2019). Desulfurization of coal using four ionic liquids with [HSO4]-. Fuel 236, 1181-1190.

582. Zhang, N., Ejtemaei, M., Nguyen, A. V, and Zhou, C. (2019). XPS analysis of the surface chemistry of sulfuric acid-treated kaolinite and diaspore minerals with flotation reagents. Miner. Eng. 136, 1-7.

583. Hayes, W.I., Joseph, P., Mughal, M.Z., and Papakonstantinou, P. (2014). Production of reduced graphene oxide via hydrothermal reduction in an aqueous sulphuric acid suspension and its electrochemical behaviour. J. Solid State Electrochem. 19, 361-380.

584. Fujimori, T., Kurisaki, K., Okobira, T., Uezu, K., and Yoshimura, K. (2011). Kinetics and molecular orbital calculations of desulfonation of strong acid cation-exchange resin. Polym. Degrad. Stab. 96, 1516-1521.

585. SantosMiranda, M.E., Marcolla, C., Rodriguez, C.A., Wilhelm, H.M., Sierakowski, M.R., BelleBresolin, T.M., and Alves de Freitas, R. (2006). I . The role of $\mathrm{N}$-carboxymethylation of chitosan in the thermal stability and dynamic. Polym Int 55, 961-969.

586. Palacios, A., Cong, L., Navarro, M.E., Ding, Y., and Barreneche, C. (2019). Thermal conductivity measurement techniques for characterizing thermal energy storage materials - A review. Renew. Sustain. Energy Rev. 108, 32-52.

587. Chung, S.H., Kim, D.H., Kim, H., Kim, H., and Jeong, S.W. (2020). Thermoelectric properties of PEDOT: PSS and acid-treated SWCNT composite 
films. Mater. Today Commun. 23.

588. Zhao, D., Qian, X., Gu, X., Jajja, S.A., and Yang, R. (2016). Measurement Techniques for Thermal Conductivity and Interfacial Thermal Conductance of Bulk and Thin Film Materials. J. Electron. Packag. 138.

589. Wijeratne, K., Vagin, M., Brooke, R., and Crispin, X. (2017). Poly(3,4ethylenedioxythiophene)-tosylate (PEDOT-Tos) electrodes in thermogalvanic cells. J. Mater. Chem. A 5, 19619-19625.

590. Zhu, Z., Liu, C., Jiang, F., Xu, J., and Liu, E. (2017). Effective treatment methods on PEDOT:PSS to enhance its thermoelectric performance. Synth. Met. 225, 31-40.

591. Hsu, J.H., and Yu, C. (2020). Sorting-free utilization of semiconducting carbon nanotubes for large thermoelectric responses. Nano Energy 67, 104282.

592. Wei, Q., Uehara, C., Mukaida, M., Kirihara, K., and Ishida, T. (2016). Measurement of in-plane thermal conductivity in polymer films. AIP Adv. 6 . 
$284 \mid$ P a g e 